
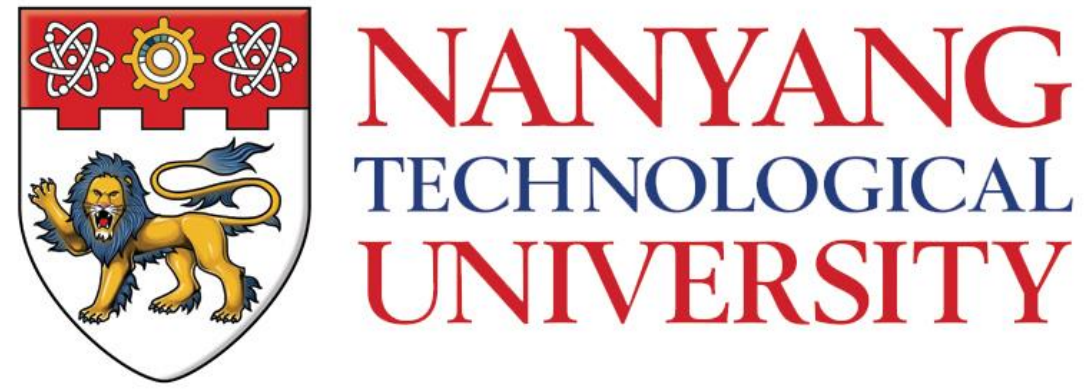

\title{
EFFECTS OF PHYSICAL STIMULI APPLIED VIA SUBSTRATE/SCAFFOLD ON IN-VITRO CULTURE OF MESENCHYMAL STEM CELLS AND NEURONS
}

XU QINWEI

SCHOOL OF MECHANICAL AND AEROSPACE ENGINEERING 



\section{EFFECTS OF PHYSICAL STIMULI APPLIED VIA SUBSTRATE/SCAFFOLD ON IN-VITRO CULTURE OF MESENCHYMAL STEM CELLS AND NEURONS}

XU QINWEI

SCHOOL OF MECHANICAL AND AEROSPACE ENGINEERING

A thesis submitted to the Nanyang Technological University in partial fulfilment of the requirement for the degree of Doctor of Philosophy 



\section{ACKNOWLEDGEMENT}

I would like to express my sincere gratitude to Dr. Zhang Yilei, my supervisor, who guided, encouraged, and supported me through my Ph.D. education. Without his expertise, the completion of this study is impossible.

I would also show my thanks to my colleagues and research fellows in our group for their support in my work, especially Dr. Jin Lin, Dr. Wang Yongdong, Ms. Li Cheng, Mr. Yi Zhengkun, Mr. Shreyas Kuddannaya and Ms. Bao Jingnan. I have received their support and had a wonderful time with them in such a beautiful place.

I thank NTU for supporting my Ph.D. study, and I appreciate the lab technicians, especially Ms. Heng Chee Hoon, in School of MAE for their kindness and help in these days.

I would like to thank my wife Ms. Li Chai (李钗) for her accompany and encouragement during my four years Ph.D. study. Sincere thanks to my grandparents, Mr. Xu Zhongcai (徐仲才), who passed away in 2006, and Ms. Zhou Lingyun (周玲云), who is suffering from Alzheimer's disease, for raising me to who I am today. Last but not least, I would like to appreciate my parents, parents in law, and all of the other family members for their endless support and love. 


\section{Table of Contents}

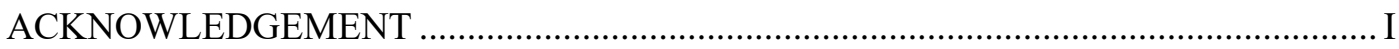

Table of Contents ...................................................................................................... III

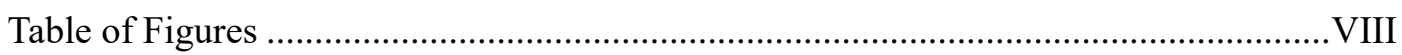

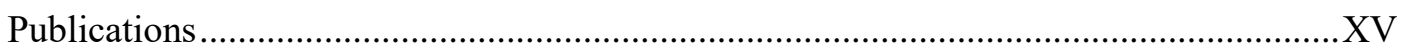

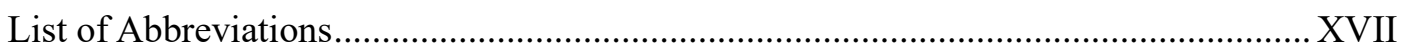

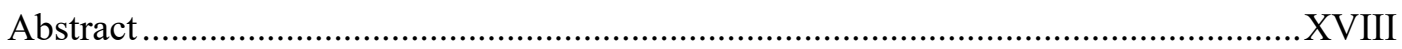

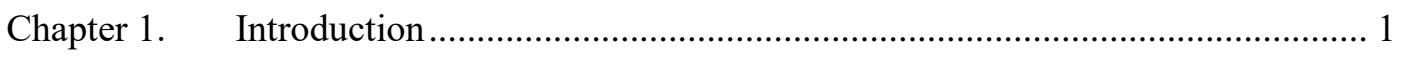

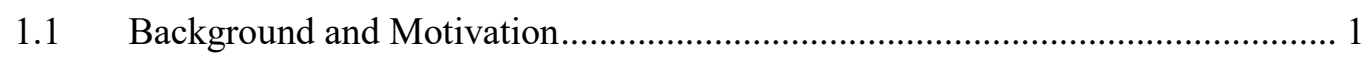

1.1.1 Stem Cells and Neurons in Tissue Engineering ...................................... 4

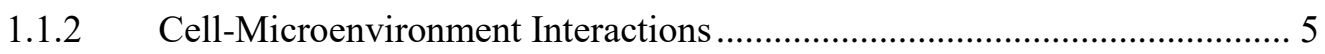

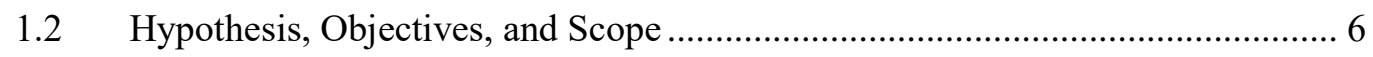

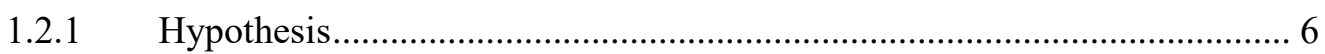

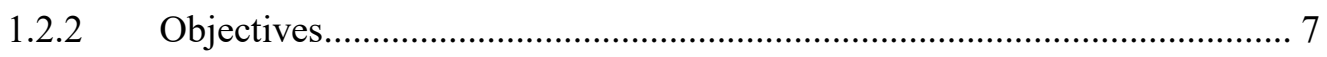

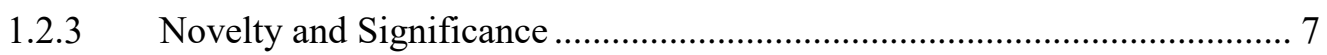

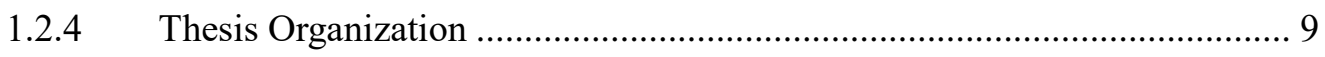

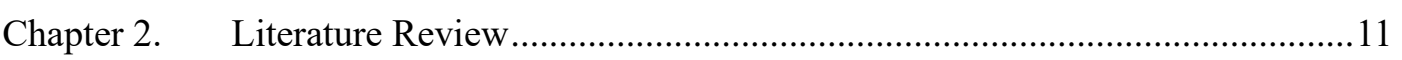

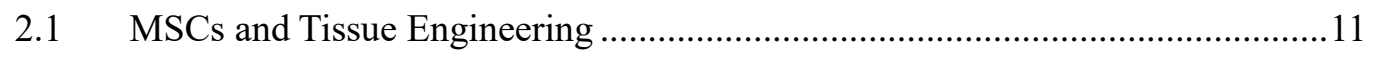

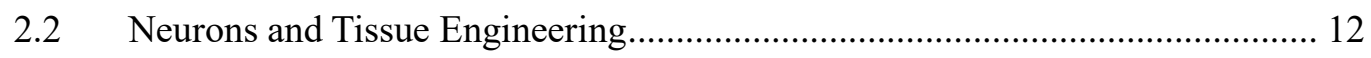




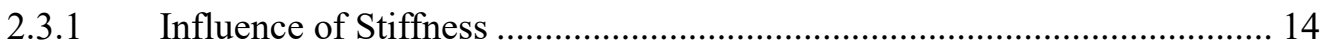

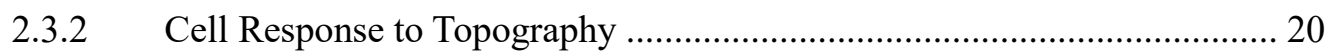

2.3.3 Effects of Mechanical Strain ................................................................ 25

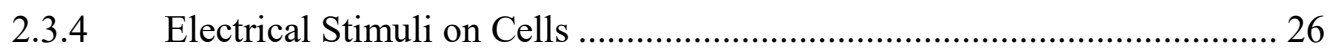

2.4 Electrospun Nanofiber and its Applications for Cell Culture ………............... 28

Chapter 3. Long Term Effects of Substrate Stiffness on the Development of hMSC

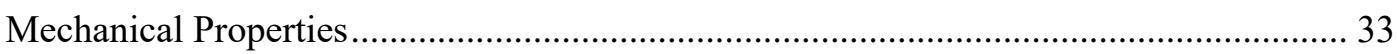

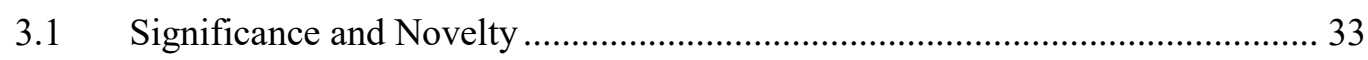

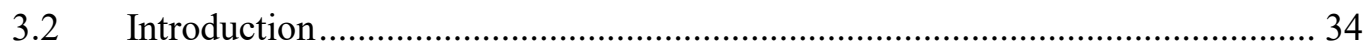

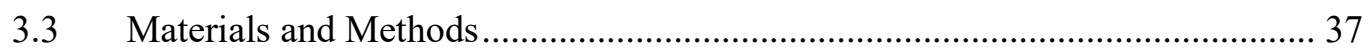

3.3.1 Fabrication of the PDMS Substrates with Different Stiffness..................... 37

3.3.2 hMSC Recovery, Expansion, and Seeding .......................................... 38

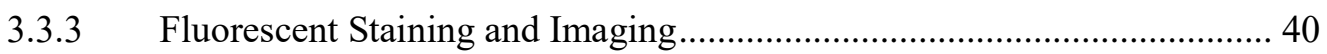

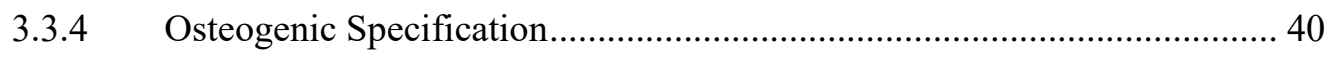

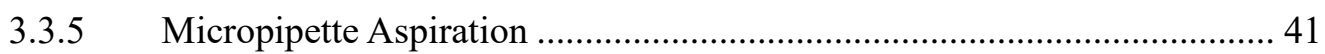

3.3.6 Theoretical Modelling ...................................................................... 43

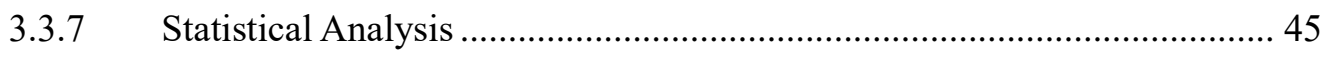

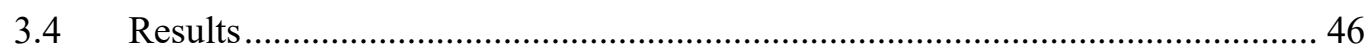


3.4.1 Stiffness of Different PDMS substrates ................................................... 46

3.4.2 Morphology of hMSCs on PDMS......................................................... 47

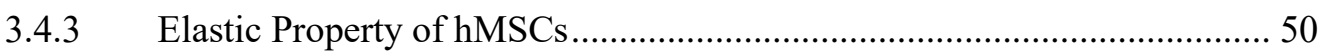

3.4.4 Viscoelastic Properties of hMSCs …….................................................. 52

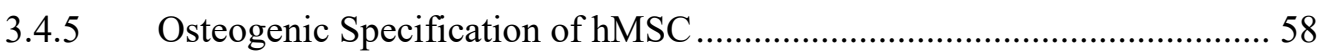

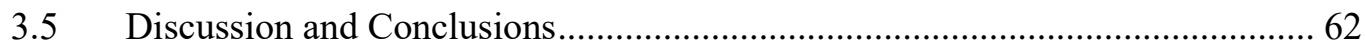

3.5.1 Cellular Mechanical Properties vs. Substrate Stiffness............................. 62

3.5.2 Cellular Mechanical Properties vs. Culture Time ..................................... 64

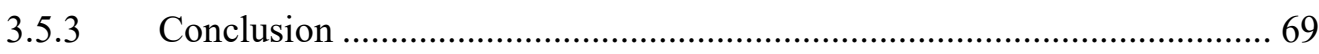

Chapter 4. Influence of Dynamic Loading on hMSCs Cultured in 3D Nanofibrous Scaffolds $\quad 72$

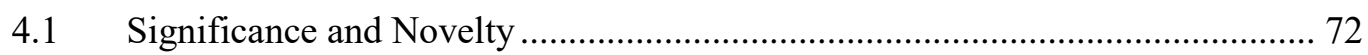

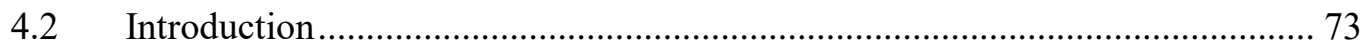

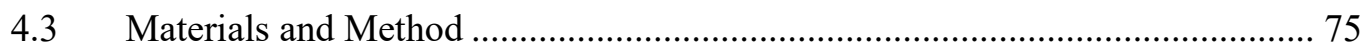

4.3.1 Fabrication of Scaffolds (2D-, 3D-NFs, and 3D-CNFs) …...................... 75

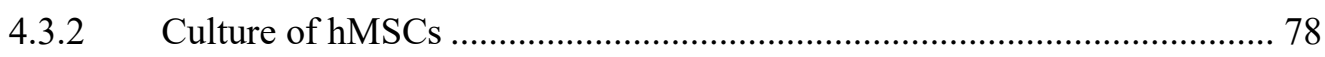

4.3.3 Fluorescent and SEM Imaging ................................................................ 79

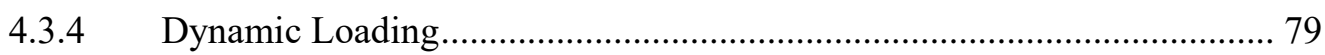

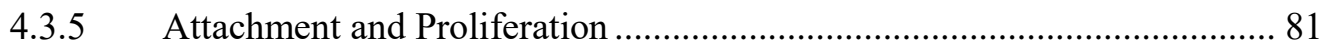




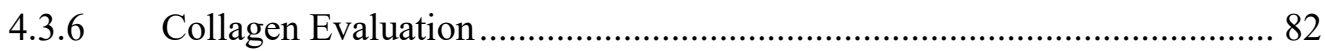

4.3.7 Characterization of Osteogenic Specification ......................................... 83

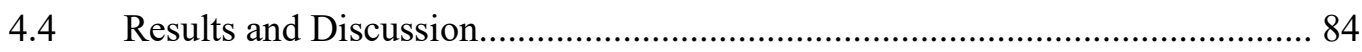

4.4.1 Structural Characterization of 2D- and 3D-NFs …................................. 84

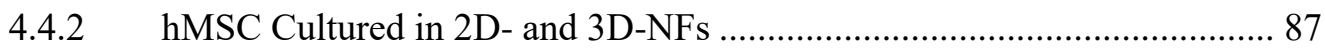

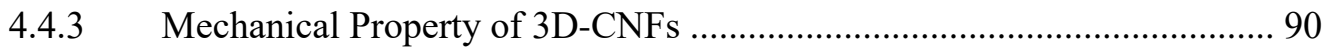

4.4.4 Cell Response to Dynamic Loading ......................................................... 92

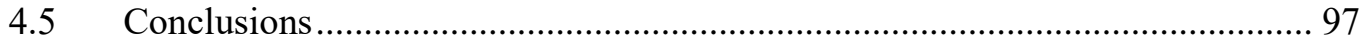

Chapter 5. Electrical Stimulation of Cortical Cells in 3D Conductive Nanofibrous Scaffolds $\quad 99$

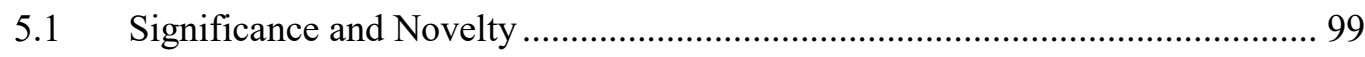

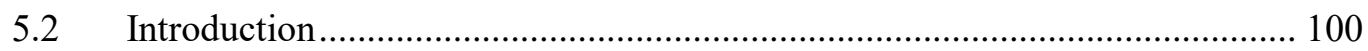

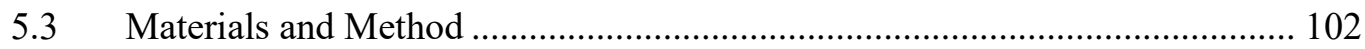

5.3.1 Fabrication of PAN nanofibers and Conductive Nanofibers ................... 102

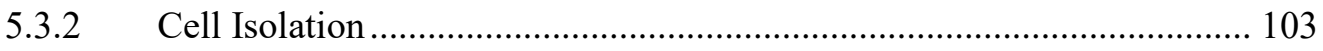

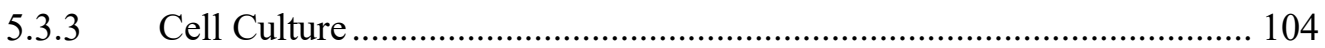

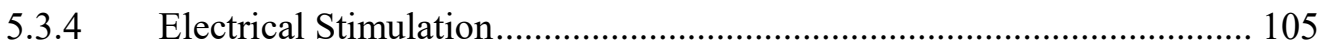

5.3.5 Immunocytochemistry and SEM Imaging ............................................ 105

5.3.6 Proliferation and Maturation .................................................................. 107 


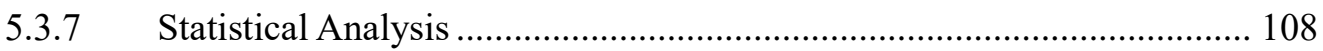

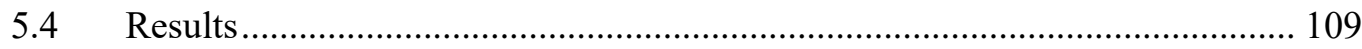

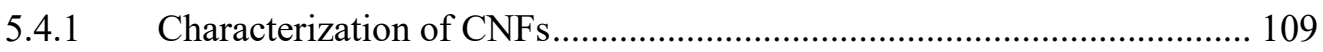

5.4.2 Cell Culture in CNFs and PAN nanofibers ...........................................111

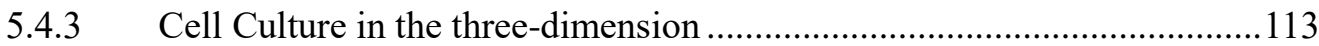

5.4.4 Effect of ES on Cell Morphology .........................................................116

5.4.5 Effect of ES on Proliferation of Cortical Cells..........................................119

5.4.6 Effect of ES on Maturation of Neurons ................................................. 121

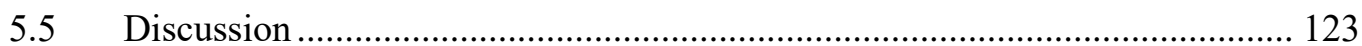

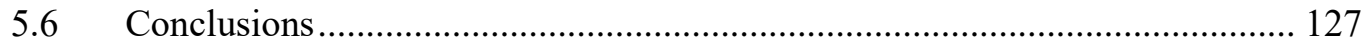

Chapter 6. General Conclusions and Future Work .................................................... 128

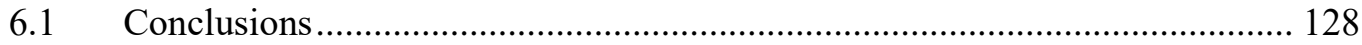

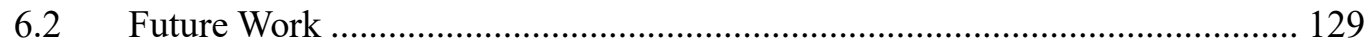

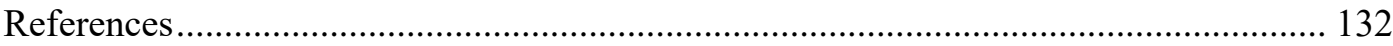




\section{Table of Figures}

Figure 1-1 Increase in number of publications in tissue engineering field

Figure 1-2 Different microenvironmental stimuli that influence cell behaviors

6

Figure 1-3 Scope of study

9

Figure 2-1 Differentiation potential of MSCs. hMSCs can differentiate into different kinds of cells (e.g., chondrocytes, osteocytes, myocytes, adipocytes, neurons)

Figure 2-2 Illustration of a tissue-engineered neural scaffold, consisting of a nerve guidance conduit (NGC) and intraluminal filament, which promotes and guides axonal growth and migration of Schwann cell. 13

Figure 2-3 Tissue stiffness and its effect on the MSC differentiation. (a) Tissues have a range of elasticity characterized by the elastic modulus, E. (b) MSCs developed different morphology when grown on matrices in the typical range of $\sim \mathrm{E}_{\text {brain, }} \sim \mathrm{E}_{\text {muscle }}$, or $\sim \mathrm{E}_{\text {bone }}$ respectively, scale bar is $20 \mu \mathrm{m}$. (c) $\beta 3$ tubulin (the neuronal cytoskeletal marker), MyoD1 (the muscle transcription factor), and $\mathrm{CBF} \alpha 1$ (the osteoblasts transcription factor) are only expressed on the neurogenic (soft), myogenic, and osteogenic matrices (stiff), respectively; scale bar is $5 \mu \mathrm{m}$. Reprinted (adapted) from Cell, Engler et al., Vol 126, Matrix Elasticity Directs Stem Cell Lineage Specification, 677-689, Copyright (2006), with permission from Elsevier ${ }^{70}$ 15

Figure 2-4 Effects of mechanical stimulation by pre-culturing cells on a stiff substrate for (b) 1, (c) 7, and (c) 10 days prior to seeding on soft substrate. (b) With 1-day pre-culture, the activation of YAP and RUNX2 was fully reversible after softening, and (c) it became partially recoverable with the 7-day pre-culture. (d) With the 10-day pre-culture, the 
activation was irreversible after softening. Reprinted by permission from Macmillan Publishers Ltd: [Nature Materials] $]^{72}$, copyright (2014)

Figure 2-5 Different elastic moduli result in the same trend of cell's phenotype as (Figure 2-3) for PAAm substrates, but no influence on cells cultured on polydimethylsiloxane (PDMS). Reprinted (adapted) by permission from Macmillan Publishers Ltd: [Nature Materials $]^{76}$, Copyright (2012). 19

Figure 2-6 (A) Axon growth along a horizontally imprinted pattern (200 nm width, $400 \mathrm{~nm}$ pitch). The arrow marked the border of the pattern. Axon growth on the ridge edges, and not in the grooves. (B): $100 \mathrm{~nm}$ width, $500 \mathrm{~nm}$ pitch; (C): $400 \mathrm{~nm}$ width, $800 \mathrm{~nm}$ pitch. Reprinted from Biomaterials, Vol. 27, F. Johansson et al., Axonal outgrowth on nano-imprinted patterns, 1251-1258, Copyright (2006), with permission from Elsevier ${ }^{98} .22$ Figure 2-7 EBL patterned structures alter gene expression. Osteogenesis (white arrows) was found to be stimulated in the disordered surface. Reprinted (adapted) by permission from Macmillan Publishers Ltd: [Nature Materials] ${ }^{102}$, Copyright (2007) 23

Figure 2-8 Differentiation of MSCs into neural lineage in different substrates, characterized by MAP2 expression. Reprinted (adapted) from Experimental Cell Research, E. Yim et al., Vol. 313, Synthetic nanostructures inducing differentiation of human mesenchymal stem cells into neuronal lineage, 1820-1829, Copyright (2007), with permission from Elsevier ${ }^{103}$ 24

Figure 2-9 Illustration of typical electrospinning setup. 29

Figure 3-1 Micropipette aspiration system illustration. 43

Figure 3-2 Illustration of the viscoelastic model, where $k 1$ and $k 2$ are the elastic 
constants of two springs; $\mu$ is the viscosity of a dashpot.

Figure 3-3 Young's modulus of PDMS measured by Instron machine (* indicates significant difference between the two groups with $\mathrm{p}<0.05,{ }^{*}$ for $\mathrm{p}<0.01$, and $* * *$ for $\mathrm{p}<0.001)$ 47

Figure 3-4 Fluorescent images of hMSC cultured on bare PDMS10 (Left) and PLL-treated PDMS10 (Right) for one day. Red: Rhodamine Phalloidin stained F-actin; Blue: DAPI stained nuclei. Scale bar: $100 \mu \mathrm{m}$ 48

Figure 3-5 Fluorescent images of hMSC cultured on PDMS. Left: Day 5 (a), Day 11 (b) and Day 14 (c) on PDMS5; Right: Day 5 (d), Day 11 (e) and Day 14 (f) on PDMS30. Red: Rhodamine Phalloidin stained F-actin; Blue: DAPI stained nuclei. 49

Figure 3-6 Young's modulus of cells calculated using elastic model ((A) modulus changing with time, (B) modulus changing with PDMS stiffness) $\left(*: \mathrm{p}<0.05,{ }^{* *}: \mathrm{p}<0.01\right.$, $* * *: \mathrm{p}<0.001$ and $\#: \mathrm{p}<0.1)$ 51

Figure 3-7 Aspiration of cells in micropipette at different time points. (A) the pressure was adjusted to reach equilibrium, (B), (C), and (D) were $0 \mathrm{~s}, 100 \mathrm{~s}$, and $300 \mathrm{~s}$ after applying the step aspiration pressure, and (E) was the corresponding fitting curve of the cell aspiration length as a function of time. 54

Figure 3-8 Cell viscoelastic parameters influenced by PDMS substrate stiffness ((A), (B), and $(\mathrm{C})$ were figures for $E 0, E e q m$, and $\mu$, respectively as functions of culture days) $(*$ : $\mathrm{p}<0.05, * *: \mathrm{p}<0.01, * * *: \mathrm{p}<0.001$ and $\#: \mathrm{p}<0.1)$ 56

Figure 3-9 Cell viscoelastic parameters as influenced culture time ((A), (B), and (C) were figures for, E0, Eeqm, and $\mu$, as functions of PDMS stiffness) $(*: \mathrm{p}<0.05, * *: \mathrm{p}<0.01$, 
$* * *: \mathrm{p}<0.001$ and $\#: \mathrm{p}<0.1)$

Figure 3-10 Osteogenic specification with culture time. (a) RUNX2 expression on PDMS5

(Day 1, Day 11 and Day 14) and Positive Control Group (cells cultured on PDMS5 with osteogenic medium on Day 14). Red: RUNX2; Blue: DAPI stained nuclei. Assessment of biomineralization on PDMS5 on day 1 (b), day 11 (c), day 14 (d) and on Positive Control Group on day 14 (e) revealed by Alizarin red staining. The arrow indicates some cell aggregations. Scale bar: $100 \mu \mathrm{m}$. 60

Figure 3-11 Percentage of RUNX2 expression on different PDMS substrates (Day 11, Day 14) and corresponding positive control groups (Day 14). All values were mean \pm standard deviation. $* * *$ indicates $\mathrm{p}<0.001$. 62

Figure 3-12 MSCs cultured on PDMS5 ((a), (c), (d)) and PDMS30 (b) on day 11. The cells had poor spreading were marked by white arrows. Red: Rhodamine Phalloidin stained F-actin; Blue: DAPI stained nuclei. (c) and (d) showed the F-actin only. Scale bar is 100 $\mu \mathrm{m}$. 64

Figure 3-13 F-actin staining of MSCs show cytoskeletal coarsening with increasing culture (Day1, Day11 and Day14) time on different substrates (TCP, PDM5 and PDMS30). 66

Figure 3-14 F-actin structure of MSCs without RUNX2 expression (a) and differentiated Osteoblast-like cells (b) cultured on PDMS10 for 14 days. Red: Rhodamine Phalloidin stained F-actin; Blue: DAPI stained nuclei; Green: RUNX2. Scale bar: $100 \mu \mathrm{m}$. 68

Figure 4-1 Schematic diagram of the fabrication steps for 2D-NFs (a) and 3D-NFs (b). (1) Electrospinning nanofibers into ethanol solution. (2) The obtained mixed solution was replaced with DI water by adding DI water 3-5 times with rigorous shaking. (3) 3D-NFs 
prepared by freeze drying.

Figure 4-2 Optical photograph of freeze dried 3D-CNFs and 3D-NFs 78

Figure 4-3 Schematic of a bioreactor for dynamic loading. 81

Figure 4-4 Optical photographs and SEM images of 2D-NFs collected from the Aluminum foil (a-b), and 3D-NFs $(0.5 \mathrm{mg} / \mathrm{mL})$ collected in the ethanol (c-d). 84

Figure 4-5 (a) Schematic description of the changes in the internal micro-porous structure with varying nanofiber $\mathrm{M} / \mathrm{V}$. (b) The microscopic architecture of the 3D NFs at various densities, they are named sample $1(\mathrm{~S} 1,0.5 \mathrm{mg} / \mathrm{mL})$, sample $2(\mathrm{~S} 2,0.25 \mathrm{mg} / \mathrm{mL})$ and sample 3 (S3, $0.125 \mathrm{mg} / \mathrm{mL})$. The insets are their optical photographs. Scale bar: $10 \mu \mathrm{m} .87$ Figure 4-6 (a-c) Fluorescent images of hMSCs on day 5 on TCPs, 2D-NFs, and 3D-NFs. (d-f) SEM images of hMSCs on day 5 on TCPs, 2D-NFs and 3D-NFs. (g) The attachment and (h) proliferation of hMSCs cultured for certain days on the TCPs, 2D-NFs and 3D-NFs. Proliferation values were normalized by the number of cells after one-day culture. Data are expressed as mean $\pm \mathrm{SD}(\mathrm{n}=3) ;{ }^{*}$ indicated $\mathrm{p}<0.05$ and $* *$ meant $\mathrm{p}<0.01$ 89

Figure 4-7 (a) (c) SEM image of 3D-CNFs (the inset is the optical photograph of a 3D-CNFs block). (b) (d) TEM image of single core-shell PPy nanofiber. (e)(f) Compressive strain-stress curve and optical picture of 3D-CNFs before and after compression in the air and DI water, respectively. 91

Figure 4-8 The compressive strain-stress curves of 3D-CNFs during ten cycles in the air. The axis range of compressive strain in the figure is from $-5-40 \%$, and the axis range of compressive stress in the figure is from -1-5 MPa. 92

Figure 4-9 Fluorescence images of hMSCs cultured for 14 days in 3D-CNF scaffolds in 
dynamic (a-c) and static states (d-f), the bars are $100 \mu \mathrm{m}$.

Figure 4-10 The proliferation of hMSCs cultured for 14 days onto/into TCPs and the 3D-NFs in static and dynamic states. (e) Collagen expression levels of hMSCs cultured 14 days onto/into the TCPs, 3D-NFs with the static and dynamic state. ${ }^{* *}$ indicated $\mathrm{p}<0.01 .95$ Figure 4-11 RUNX2 expression of MSCs after 14-day culture in both dynamic and static condition, as well as positive controls marked with “+”. Scale bar: $50 \mu \mathrm{m}$. 97

Figure 5-1 Schematic of (a) electrospinning setup and (b) a bioreactor for electrical stimulation (3D View and Cross-section).

Figure 5-2 Photographs and SEM images of 2D-NFs mat ((a)), 2D-CNFs ((b) (c)), 3D-NFs

(d), and 3D-CNFs scaffold ((e) (f)). 110

Figure 5-3 CV curves of 3D-CNFs at s scan rate of $50 \mathrm{mv} / \mathrm{s}$. 111

Figure 5-4 Fluorescent images of anti-MAP2-stained neurons in (a) 2D-PANs, (b) 2D-CNF nanofibers, (c) 3D-PAN and (d) 3D-CNF nanofibers. Green: MAP2; Blue: DAPI.

Figure 5-5 SEM of cortical cells cultured in 2D-CNFs (a, c), 3D-CNFs (b, d) and confocal microscopic ortho-images of cell culture in 2D-CNFs (e) and 3D-CNFs (f). The red arrows and triangles indicated two different cell morphologies respectively. Green: MAP2; Blue:

DAPI. 115

Figure 5-6 Confocal fluorescent images of neuron morphology after 7-day culture in the 3D-CNFs (a) without and (b) with electrical stimulation. Neurite connections between individual neurons and connection between clusters were marked with red lines. 116

Figure 5-7 Confocal fluorescent images of neurons and glial cells on day 7 in the 
3D-CNFs (a-d) without and (e-h) with electrical stimulation. Blue: DAPI; Green: MAP2; Red: GFAP. Neuron grew without glial cells around was marked with the red circle; cells expressed neither MAP2 nor GFAP marked with the white circles. Scale bar: $100 \mu \mathrm{m} . . .117$ Figure 5-8 Immunostaining of synaptophysin and PSD 95 in 7-day cultured cortical cells in Control (a)(b) and ES (c)(d) groups. (b) and (d) were regions of interest (indicated with dashed line) from (a) and (c) respectively. The synaptophysin was detected adjacent to PSD 95 (indicated with arrows) 119

Figure 5-9 Proliferation (represented by relative absorbance in $570 \mathrm{~nm}$ ) of cell cultures with or without electrical stimulation in day 1 , day 3 and day 7. * indicated $\mathrm{p}<0.05$, indicated $\mathrm{p}<0.01, * * *$ indicated $\mathrm{p}<0.001$ and $* * * *$ indicated $\mathrm{p}<0.0001$

Figure 5-10 Maturation of neurons with or without electrical stimulation in day 1, day 3 and day 7. * indicated $\mathrm{p}<0.05, * *$ indicated $\mathrm{p}<0.01, * * *$ indicated $\mathrm{p}<0.001$ and indicated $\mathrm{p}<0.0001$

Figure 5-11 Surface roughness of PAN (a) and PPy-coated PAN nanofibers (b). *(a) was reproduced from Ref. ${ }^{338}$ with permission of RSC Advances. 


\section{Publications}

Journal Paper:

(1) Jin, L.; Xu, Q.; Kuddannaya, S.; Li, C.; Zhang, Y.; Wang, Z. Fabrication and Characterization of Three-Dimensional (3D) Core-Shell Structure Nanofibers Designed for 3D Dynamic Cell Culture. ACS Appl. Mater. Interfaces 2017, 9 (21), 17718-17726.

(2) Jin, L.; Xu, Q.; Li, C.; Huang, J.; Zhang, Y.; Wu, D.; Wang, Z. Engineering 3D Aligned Nanofibers for Regulation of Cell Growth Behavior. Macromol. Mater. Eng. 2017, 302 (4), 1-9.

(3) Jin, L.; Xu, Q.; Wu, S.; Kuddannaya, S.; Li, C.; Huang, J.; Zhang, Y.; Wang, Z. Synergistic Effects of Conductive Three-Dimensional Nanofibrous Microenvironments and Electrical Stimulation on the Viability and Proliferation of Mesenchymal Stem Cells. ACS Biomater. Sci. Eng. 2016, 2 (11), 2042-2049.

(4) Jin, L.; Ren, K.; Xu, Q.; Hong, T.; Wu, S.; Zhang, Y.; Wang, Z. Multifunctional Carbon Dots for Live Cell Staining and Tissue Engineering Applications. Polym. Compos. 2016, 16 (2).

(5) Xu, Q.; Li, C.; Kang, Y.; Zhang, Y. Long Term Effects of Substrate Stiffness on the Development of hMSC Mechanical Properties. RSC Adv. 2015, 5 (128), 105651-105660.

(6) Xu, Q.; Wang, Y.; Siew, S. Y.; Lin, J.; Zhang, Y. Generating Self-Accelerating Airy Beams Using a Digital Micromirror Device. Appl. Phys. B 2014, 117 (1), 141-144. 


\section{Conference Paper:}

(1) Badjo, W.; Li, C.; Xu, Q.; Kuddannaya, S.; Zhang, Y.; Kang, Y. Cellular Stiffness Measurement for 3D Biological Printing. In Proceedings of the 1st International Conference on Progress in Additive Manufacturing; Research Publishing Services: Singapore, 2014; pp 417-422. 


\section{List of Abbreviations}

\begin{tabular}{|c|c|}
\hline Full Name & Abbreviation \\
\hline Human Mesenchymal Stem Cells & hMSCs \\
\hline Human Embryonic Stem Cells & hESCs \\
\hline Extracellular Matrix & ECM \\
\hline Nanofibers & NFs \\
\hline Conductive Nanofibers & CNFs \\
\hline Polypyrrole & PPy \\
\hline Electrical Stimulation & ES \\
\hline Peripheral Nervous System & PNS \\
\hline Central Nervous System & $\mathrm{CNS}$ \\
\hline Polydimethylsiloxane & PDMS \\
\hline Microtubule-Associated Protein 2 & MAP2 \\
\hline Arginylglycylaspartic acid & RGD \\
\hline Fetal Bovine Serum & FBS \\
\hline Phosphate Buffered Saline & PBS \\
\hline Dulbecco's Modified Eagle's Medium & DMEM \\
\hline
\end{tabular}




\section{Abstract}

Interactions between cells and the microenvironment have been demonstrated to be key factors in cell behavior regulation. Among the different cues provided by the microenvironment, the physical cues from the microenvironment are emerging at a rapid pace and have attracted a lot of attention. However, the studies in this area have not yet been comprehensive, and many phenomena and mechanisms have still not been completely clarified. For example, the effects caused by physical stimuli could be complex considering other factors, such as culture time, three-dimensional (3D) structure of scaffolds, etc. This Ph.D. project was proposed to obtain a better understanding of cell-microenvironment interactions with physical stimuli, and three sub-projects were conducted specifically for this purpose.

In the first part, the mechanical properties of human mesenchymal stem cells (hMSCs) were found to be influenced by both the mechanical properties of the substrates that they adhered to and the culture time. The results showed that cells well aligned their mechanical properties according to the substrate stiffness and cell moduli as functions of time displayed non-monotonic trend. This project proved the concept of regulating cell modulus through modifying cell growth substrates and culture time.

In the second part, the effect of dynamic loading on hMSCs in a 3D scaffold was investigated. A 3D nanofibrous scaffold (3D-CNFs) was fabricated using a novel method for creating a 3D cell culture. These conductive PPy-coated 3D-NFs (3D-CNFs) showed super-elastic properties, which supported the dynamic cell culture of hMSCs. It was found that cell penetration and adhesion were supported by 3D-CNFs. In the condition of 
dynamic compressive loading, the morphology and collagen expression of hMSCs in 3D-CNFs were changed. It provided a new way to build a dynamic 3D cell culture to studying cell-microenvironment interactions.

The last part studied the effect of electrical stimulation (ES) applied on neurons and glial cells in the 3D-CNFs. It was conducted to address the combined effect of 3D-CNFs scaffolds and ES on cell cultures. 3D-CNFs with 3D structures successfully supported the 3D culture of cortical cells. ES changed the morphology and distribution of cells. ES also increased the proliferation of cortical cells in the 3D-CNFs significantly. Furthermore, the maturation of neurons in 3D-CNFs was promoted significantly by ES compared to unstimulated neurons starting from the first day of culture. These combined effects indicated that ES and 3D-CNF have broad applications in neural engineering, such as implantation and biofunctional in vitro models. 


\section{Chapter 1. Introduction}

\subsection{Background and Motivation}

Millions of people are suffering from soft organ diseases and physical disabilities all over the world. Organ transplantation is one of the ways to address these issues. However, the gap between demand and supply is still huge, and it is becoming larger and larger. The limited supply of organs is not only destroying many patients' hope, but also causing serious bioethics issues and crimes, including organ trade, organ tourism, and organ harvesting. To overcome this crisis, an alternative source of organ transplantation is required.

Tissue engineering, according to the definition of Langer and Vacanti, is "an interdisciplinary field that applies the principles of engineering and life sciences toward the development of biological substitutes that restore, maintain, or improve tissue function

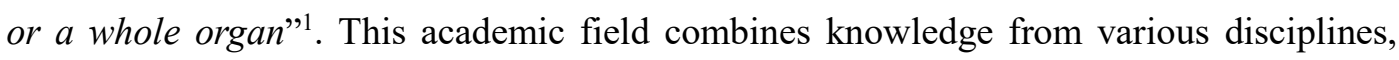
including biology, chemistry, physics, medicine, materials science, and engineering, and applies it and related technology to clinical applications, including organ repair and regeneration.

Tissue engineering has attracted a lot of interest due to the need for the repair and regeneration of damaged tissues in recent decades. The interest in tissue engineering is reflected in the number of relevant publications, which have dramatically increased in the last two decades, as illustrated in Figure 1-1. A PubMed search for "Tissue Engineering" reveals over 37,000 papers with a substantial increase in the number of publications every 
year. Starting from only around 50 publications in 1996, there were over 4,000 in 2015.

Tissue engineering research consists of three stages: The first stage focuses on the implantation of cultured or isolated cells, the second stage focuses on tissues built in vitro by cells-laden scaffolds, and the third stage focuses on tissue regeneration in situ ${ }^{1,2}$. The key factor in tissue engineering is how to control and regulate cell behaviors, (e.g., migration, proliferation, differentiation) to reconstruct new organs.

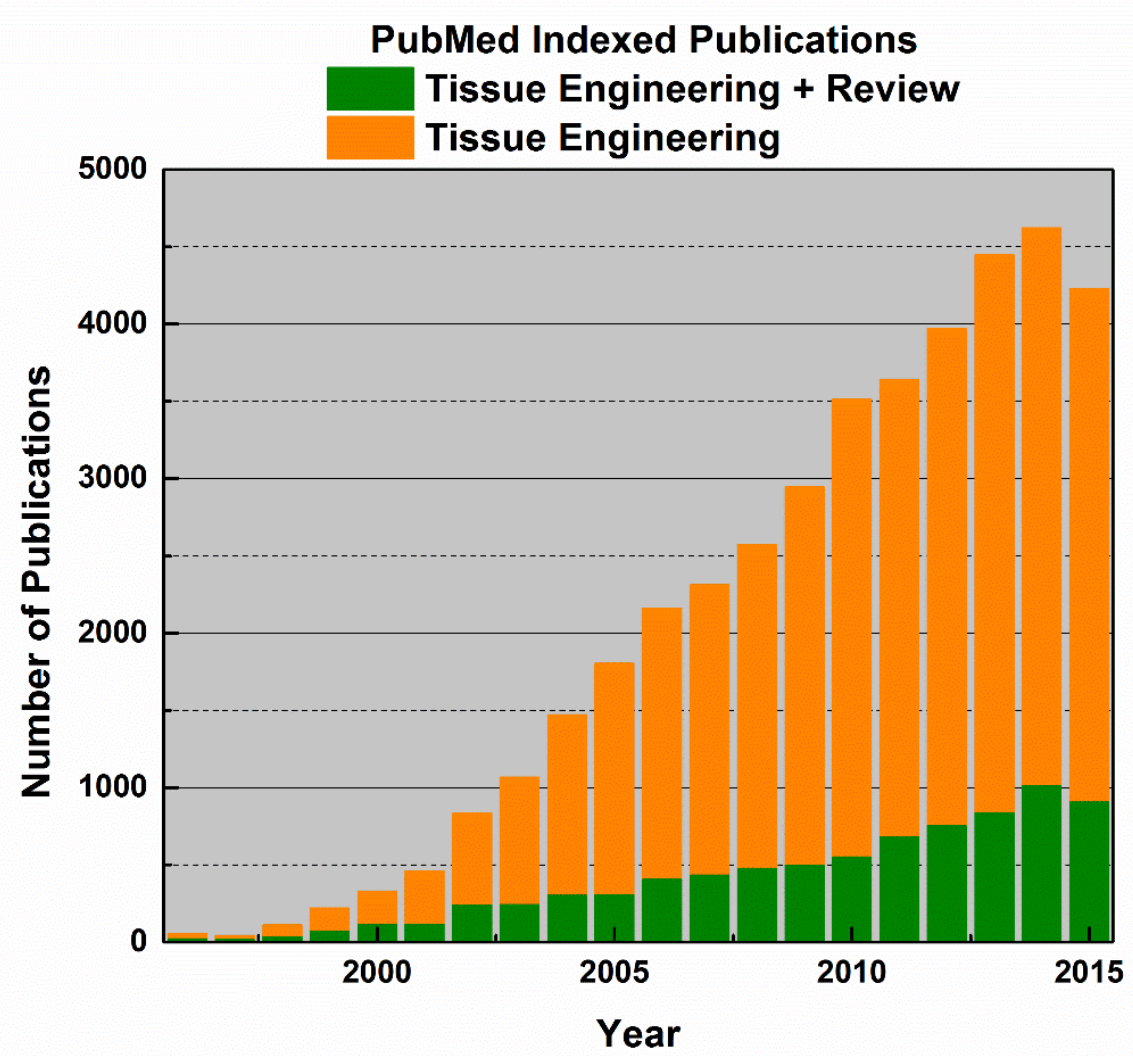

Figure 1-1 Increase in number of publications in tissue engineering field

Source: PubMed (https://www.ncbi.nlm.nih.gov/pubmed)

Human mesenchymal stem cells (hMSCs) are multipotent stem cells, isolated mainly from adult bone marrow $(\mathrm{BM})^{3}$. The reason for the interest in studying MSCs lies in their ability to differentiate into different cell lineages as well as their potentials in cell and gene therapy for various diseases, like heart failure and hematological pathologies ${ }^{4}$. Neurons 
are another important cell type studied in tissue engineering. The motivation for neuron studies stems from the fact that millions of people are affected by nervous system injuries worldwide every year. Severe nerve system injuries may result in life-long disabilities in patients as well as serious economic and social problems ${ }^{5}$. Because the regenerative capability of the adult nervous system is often limited, one alternative approach in nervous system treatment is to build cell-laden scaffolds in vitro for implantation ${ }^{6-10}$. It has been demonstrated that stem cells and neurons can be influenced by their environments. Many materials and techniques have been developed to induce biological, chemical, and even biophysical cues to control cell behaviors, including differentiation, proliferation, adhesion, and migration. Compared with biological and chemical stimuli, methods to induce biophysical stimuli have their own advantages, like ease of control and fewer side effects as compared to those from biological and chemical factors ${ }^{11}$. Recent studies have shown that substrate stiffness is an important factor in cell culture, because it can be sensed by cells from the environment ${ }^{12,13}$. Furthermore, surface topography, mechanical stimulation, surface patterning, and electrical stimulation (ES) have also been utilized to investigate cell behaviors ${ }^{14-20}$.

While emphasis has been placed on how to control cell behaviors, the mechanism of cell-microenvironment interactions is still not completely clear, but it is definitely important. We believe the study of cell behavior control via substrates/scaffolds could enrich the understanding of cell-microenvironment interactions and enlighten future studies on biomaterials and tissue engineering. 


\subsubsection{Stem Cells and Neurons in Tissue Engineering}

Tissue engineering aims to rebuild or replace the biological functions of tissues to treat human disease through combining multiple disciplines, including engineering, chemistry, cell biology, and materials science ${ }^{1,2,21-24}$. To achieve this goal, studies on cooperation of stem cells and differentiated cells, like neurons, are now under investigation to provide clinical treatment alternatives.

Stem cells can differentiate into numerous cell lineages and self-renew for population growth $^{25}$. The variety of cell types they can differentiate into is determined by the stem types (e.g. pluripotent stem cells, multipotent stem cells, or unipotent stem cells) ${ }^{26}$. These characteristics make all stem cells promising in tissue engineering. Among them, MSCs have attracted great interest. The first discovery of MSCs was in $1968^{27}$, and the use of MSCs in clinical trials started in $1995^{28}$. As of July 2016, there are 557 clinical trials using MSCs are recorded in www.clinicaltrials.gov for various clinical applications. However, most of them are still in Phase 0 (safety studies in a small group, $n=9$ ), Phase I (safety studies, $\mathrm{n}=161$ ), Phase II (efficacy studies, $\mathrm{n}=138$ ), or combined Phase I and II studies $(n=220)$. Only a small number of trials are Phase III (comparing to existing treatment, $n$ $=25)$, combined Phase II and III $(\mathrm{n}=20)$, or Phase IV studies (approved by FDA, $\mathrm{n}=3$ ). This indicates there is still a long way to go.

Neurons are another important cell type attracting a lot of interest in tissue engineering. It has demonstrated that utilizing tissue-engineered nerve grafts to bridge peripheral nerve defects is a promising alternative to autologous nerve grafts ${ }^{6,29-34}$. Researchers have concluded some criteria for a practical nerve graft $^{30}$ : (1) controllable rate of 
biodegradation; (2) biocompatible with cells and nerve tissue; (3) reduced immunogenicity; (4) suitability for angiogenesis and metabolism; (5) reduced scar formation; (6) high availability of scaffold materials. Furthermore, these grafts should provide a biomimetic microenvironment that allows neurons to grow neurites, and guide the orientation. In recent decades, various synthetic and natural biomaterials have been studied as scaffolds used in nerve tissue engineering ${ }^{35}$. However, neural tissue engineering is still a growing field that remains to be explored for clinical use.

\subsubsection{Cell-Microenvironment Interactions}

The cell behaviors of both stem cells and neurons are regulated by various microenvironmental factors, including biochemical signals (soluble factors), physical signals, as well as cell-cell interactions. Therefore, the study of cell-microenvironment interactions is important in tissue engineering. However, most existing studies have focused on the influence of biological or chemical cues provided by the microenvironment, and therefore, side effects have been unavoidable. For example, toxic side effects are one of the common reasons for a failure of a chemical induced cell behavior regulation. Physical cues are becoming more and more attractive, and numerous biomaterials have been developed and explored as scaffolds or substrates to affect or control cell behaviors ${ }^{36-}$

41. Figure 1-2 shows that many cues or stimuli influence cell behaviors, including mechanical cues, electrical stimuli, the properties of the biomaterials themselves, as well as biological/chemical factors. In this report, emphasis placed on the roles physical cues, including mechanical cues and ES, play in cell behavior control, while the properties of 
scaffolds were also considered.

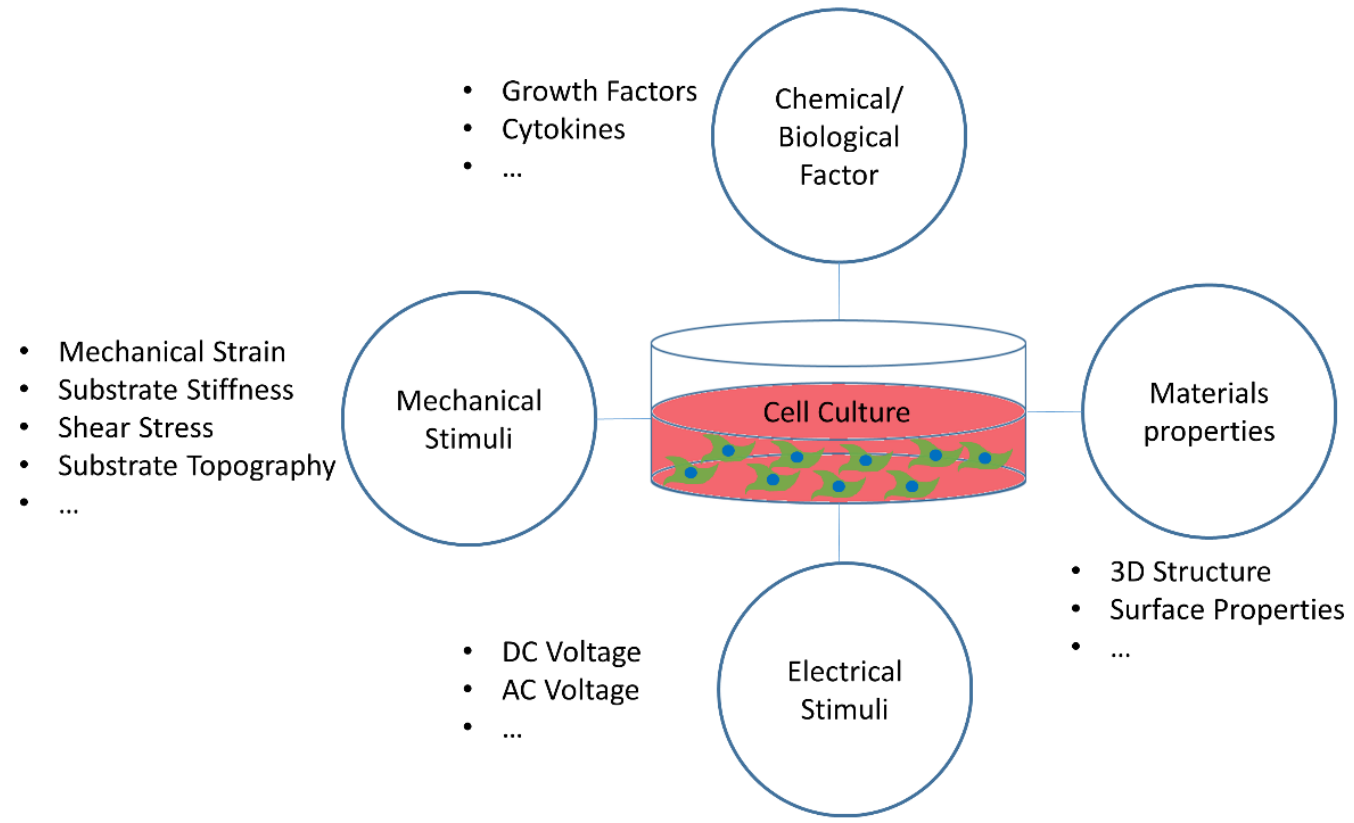

Figure 1-2 Different microenvironmental stimuli that influence cell behaviors

\subsection{Hypothesis, Objectives, and Scope}

\subsubsection{Hypothesis}

This study was inspired by previous studies of cell behavior controlled by mechanical cues and electrical cues ${ }^{26,42}$ that revealed that different kinds of mechanical and electrical cues have specific effects on certain cell behaviors, such as the differentiation of MSCs, and neurite growth of neurons. Thus, we hypothesized that the effects of physical stimuli from the microenvironment combined with other factors, like culture time, surface topography, and three-dimensional (3D) structure of scaffolds, could be more complex and provide insight into the mechanism of cell-microenvironment interactions. We contend that by controlling the stiffness of scaffolds/substrates, or applying mechanical loading or 
electrical stimuli via different kinds of scaffolds/substrates, we can alter cellular behaviors, including cell stiffness, morphology, proliferation, and specific gene expression.

\subsubsection{Objectives}

The general objective of this thesis is to investigate the combined effects of physical stimuli and other factors, including time, topography, and 3D structures, on cell behaviors. We utilize different scaffolds or substrates with specific mechanical cues or electrical stimuli to modulate cell behaviors. The specific objectives are as follows:

- Study hMSCs behaviors (morphology, elastic/viscoelastic properties) on substrates with various stiffness during a long-term culture time.

- Utilize 3D scaffolds and a customized bioreactor to induce dynamic loading to 3D hMSCs culture, and explore their effects on hMSCs behaviors (proliferation, specific gene expression, and morphology).

- Utilize 3D scaffolds and a customized bioreactor to induce ES to 3D neuron and glial cell culture and explore their effects on cell behaviors (glial proliferation, neuron maturation, and morphology)

These studies could enrich the methods of cell-microenvironment interaction studies and contribute to the state-of-the-art understanding of the underlying mechanisms.

\subsubsection{Novelty and Significance}

The influence of mechanical cues and electrical stimuli on cell behaviors (e.g., adherence, migration, proliferation) has been extensively studied in the past, but the studies have not 
been comprehensive and numerous unknown effects and mechanisms remain to be studied. Furthermore, most studies have focused on the effect caused by one kind of stimuli, and ignored some important factors. For example, some effects of physical stimuli on cells may be time-dependent. Physical stimuli applied through a 3D scaffold could be closer to native conditions, compared with stimuli applied through a 2D substrate. We hypothesized that the responses of cells to physical stimuli coming from substrates/scaffolds could be complex and different when multiple factors are considered, such as culture time and 3D structures. For example, systematical investigation of the change of hMSCs' elastic and viscoelastic properties in responding to both the stiffness of the substrates they adhered to and the culture days in vitro has been undertaken. We also studied the cellular response to the dynamic loading in a 3D electrospun scaffold. Furthermore, the responses of cortical neurons and glial cells cultured in 3D conductive scaffolds to ES were reported. This work will definitely enrich and contribute to the knowledge of materials design and culture technologies in tissue engineering. 


\subsubsection{Thesis Organization}

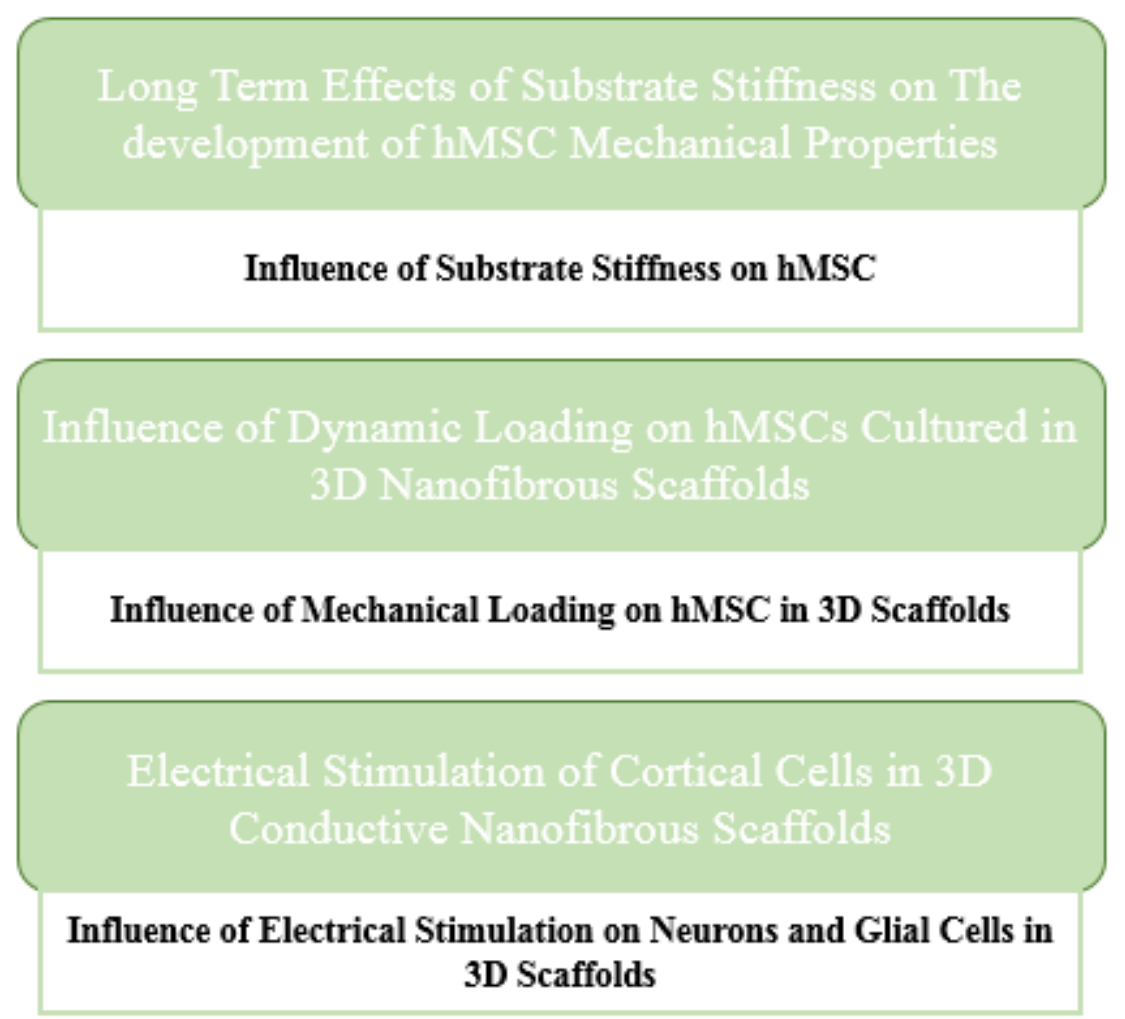

Figure 1-3 Scope of study

The organization of this thesis is briefly described in Figure 1-3. A brief introduction had been given in the first chapter to introduce the background, motivation, and objectives of this study. In the second chapter, the general trend in MSCs and neurons involved in tissue engineering was reviewed, followed by a brief discussion of the influence of different physical stimuli on cells. The development of electrospun nanofibers and their applications in cell culture were also summarized in the same chapter. Chapter $3 *$ studies the influence of substrate stiffness and culture time on the mechanical properties of hMSCs. In Chapter 4**, hMSCs were cultured in a novel 3D scaffold and stimulated with dynamic compression loading. The responses of hMSCs, including proliferation, 
morphology, and collagen expression, were discussed. In Chapter $5^{* * *}$, neurons and glial cells cultured in 3D-CNFs were electrically stimulated for a week, and the changes in morphology, glial cell proliferation, and neuron maturation were studied. A general conclusion was made and recommendations for future studies were proposed in the last chapter.

*: Chapter 3 is based on the publication of $[\underline{\mathrm{Xu}}, \mathrm{Q}$; Li, C.; Kang, Y.; Zhang, Y. Long Term Effects of Substrate Stiffness on the Development of hMSC Mechanical Properties. RSC Adv. 2015, 5 (128), 105651-105660.,]

**: Chapter 4 is based on the publication of [Jin, L.; Xu, Q.; Kuddannaya, S.; Li, C.; Zhang, Y.; Wang, Z. Fabrication and Characterization of Three-Dimensional (3D) CoreShell Structure Nanofibers Designed for 3D Dynamic Cell Culture. ACS Appl. Mater. Interfaces, 2017, 9 (21), 17718-17726.]

***: Chapter 5 is based on the publication of $[\underline{\mathrm{Xu}}, \mathrm{Q}$; Jin, L.; Li, C.; Kuddannaya, S.; Zhang, Y. Electrical Stimulation of Cortical Cells in 3D Conductive Nanofibrous Scaffolds. Submitted] 


\section{Chapter 2. Literature Review}

\subsection{MSCs and Tissue Engineering}

MSCs were first discovered in 1968 by Friedenstein and colleagues and described as adherent fibroblast-like cells in the bone marrow (BM) capable of differentiating into bones $^{27}$. They are multipotent stem cells that can be isolated mainly from BM. They can also be sourced from liver tissues, amniotic fluid, and placental tissues ${ }^{3}$. The significance of studying MSCs lies in their potential to differentiate into multiple cell lineages ${ }^{4}$, such as, myocytes, osteocytes, adipocytes, chondrocytes, and neurons, which makes them promising for use in cell and gene therapy for serious diseases, like heart failure or hematological pathologies ${ }^{3}$. These cells can be cultured and proliferate in vitro, which allows them to expand quickly and be harvested for use in vivo. Unlike embryonic stem cells (ESCs), which have only a short-time lifespan of around two years in vivo, MSCs could reside longer into adult life ${ }^{43}$, and therefore culture-expanded marrow-derived MSCs could have the capability of differentiation independent of the age of the donors ${ }^{41}$.

Based on the literature review, it is known that hMSCs are able to self-renew, and differentiate into many cell lineages (Figure 2-1), which makes hMSCs a promising source for tissue engineering, and therefore, studies of hMSCs combined with biomaterials have been pursued in tissue engineering for bones, muscles, fat, and neuron repair ${ }^{41,44}$. 


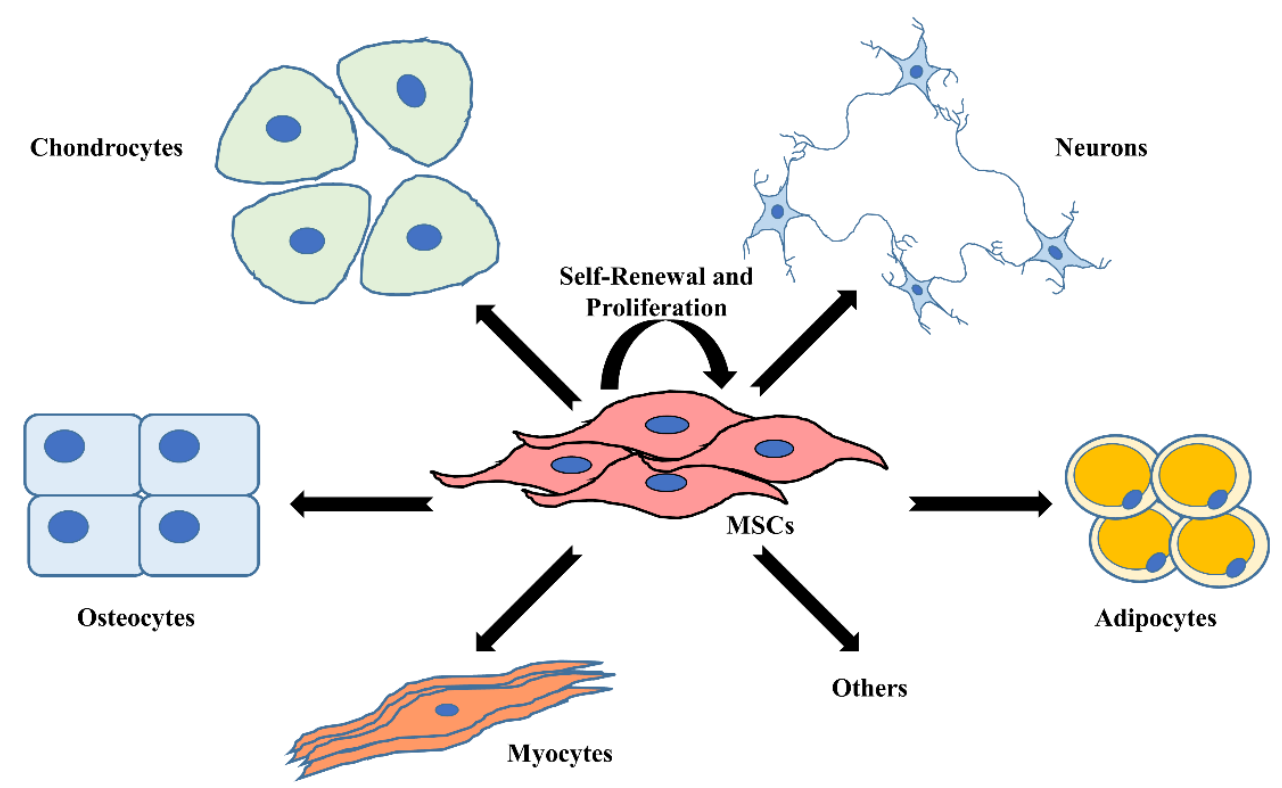

Figure 2-1 Differentiation potential of MSCs. hMSCs can differentiate into different kinds of cells (e.g., chondrocytes, osteocytes, myocytes, adipocytes, neurons).

\subsection{Neurons and Tissue Engineering}

The motivation for studies of neural tissue engineering comes from the facts that millions of people are affected by traumatic injuries to the nervous system every year, which lead to lifelong disabilities in patients, heavy economic burdens on public services, and social problems $^{45,46}$. However, neural defects are not easy to treat. Spontaneous nerve regeneration in the peripheral nervous system (PNS) has a poor functional outcome if no therapeutic interference is adopted, even though the PNS has intrinsic regenerative capabilities $^{47}$. The issue in the central nervous system (CNS) is even worse. Due to the presence of inhibitors within myelin and the scar formation, neurons in the CNS cannot regenerate on their own after nerve injury ${ }^{48,49}$. Because of the reasons mentioned above, treatment of CNS injuries remains a challenge, while clinical treatment of PNS injuries has achieved some remarkable progress ${ }^{50}$. A typical process of a tissue-engineered nerve 
bridging a nerve defect is shown in Figure 2-2. The tissue-engineered nerve can bridge the nerve defect and injured neurons can grow neurites along the nerve graft. The limitation in nerve regeneration requires new methods to improve neuron growth in vitro, e.g. applying physical stimuli.

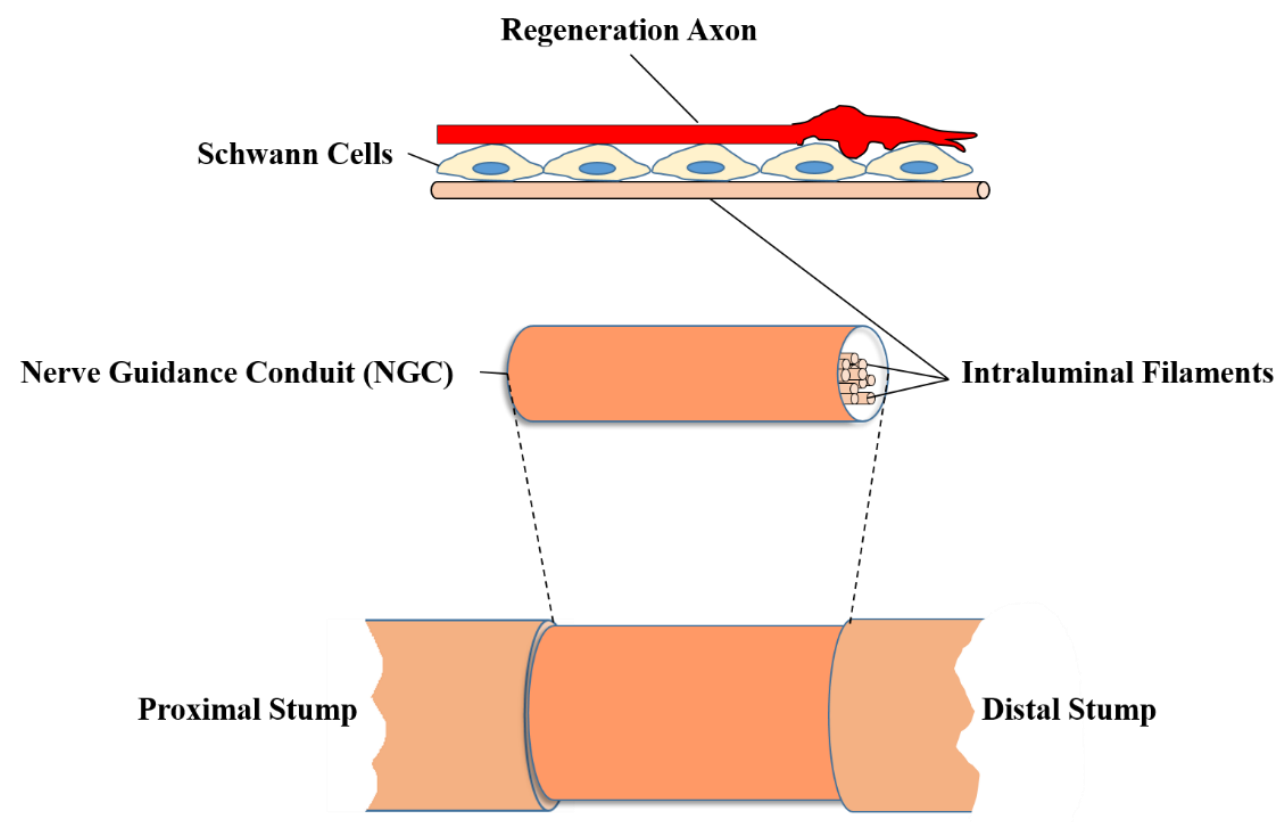

Figure 2-2 Illustration of a tissue-engineered neural scaffold, consisting of a nerve guidance conduit (NGC) and intraluminal filament, which promotes and guides axonal growth and migration of Schwann cell.

\subsection{Cell Behavior Control and Physical Cues}

The extracellular environment or the extracellular matrix (ECM) in vivo supports cells, separates one tissue from another, regulates intercellular communication, stores and releases growth factors, etc. It has been proven that the cellular behaviors of cells, including locomotion, proliferation, and differentiation (for stem cells), are controlled by a complex set of signals directed by their growing microenvironment ${ }^{11,26,51-56}$. It is well 
known that soluble factors (e.g., growth factors, cytokines) significantly influences cells in differentiation and self-renewal ${ }^{57,58}$. However, known soluble factor-mediated signaling pathways cannot explain many effects on cell behaviors. Recently, the physical signals/cues presented by the microenvironment have become attractive and been studied as essential factors regulating cell behaviors ${ }^{26,56,59}$. For example, the adhesion and alignment of cells can be influenced by a substrate's stiffness ${ }^{60}$ and flexibility ${ }^{61}$, and the growth direction can be guided by its topography and morphology ${ }^{626310}$. Furthermore, an external stimulus applied via the microenvironment, such as dynamic compression ${ }^{64}$ or an electrical stimulus ${ }^{65}$, could affect various cell behaviors, from proliferation to differentiation.

\subsubsection{Influence of Stiffness}

The influence of the stiffness of a cell's microenvironment exists in all stages of cell life, from embryogenesis ${ }^{66}$ to terminal cell differentiation ${ }^{67}$. Changes in tissue stiffness could be a signal of certain disease states. For instance, it is well-known that breast cancer tumors are more rigid than neighboring tissues because of the matrix stiffness increase caused by collagen fibrils clusters. This fact has been used for breast self-exams. It has been well-known that mammary epithelial cells cultured on unusually stiffer substrates, have poor tissue formation, and promote malignancy ${ }^{68,69}$. Therefore, stiffness change has a direct influence on cell behaviors.

In 2006, a seminal study was conducted by Engler et al. to demonstrate that the substrate stiffness could alter the differentiation of $\mathrm{MSCs}^{70}$. Three different substrates with stiffness 
of $0.1-1,8-17$, and $25-40 \mathrm{kPa}$ were utilized to mimic the elastic moduli of the brain,

(a)

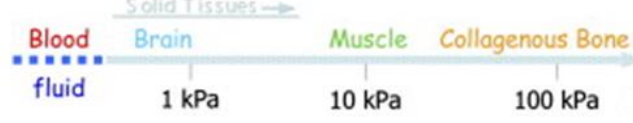

(b)
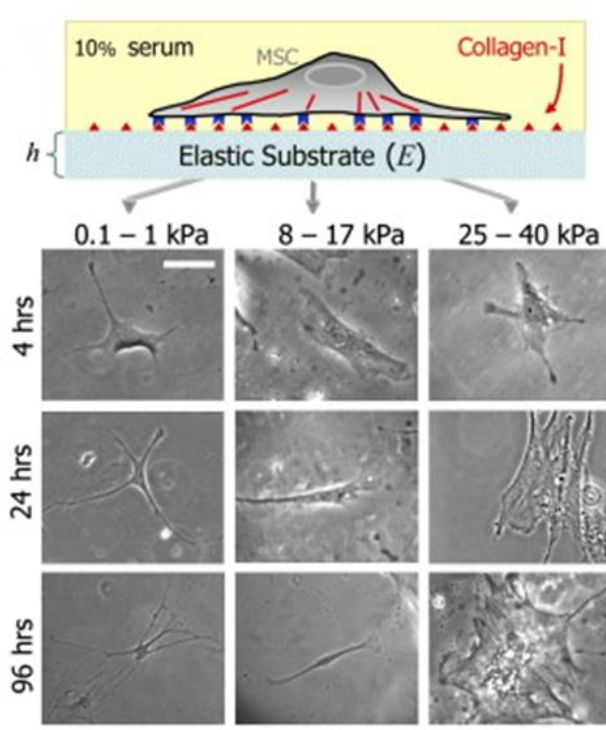

(c)
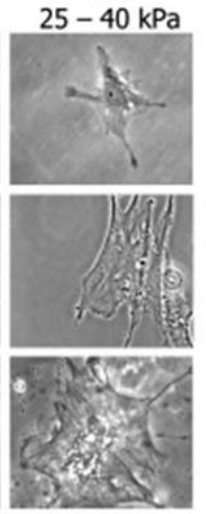
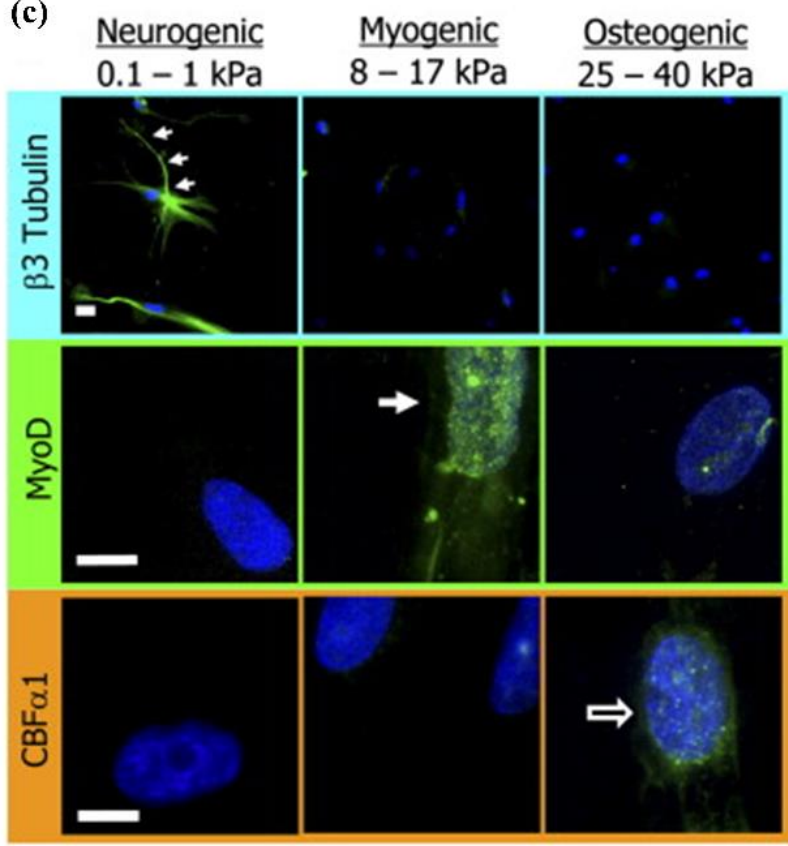

muscle, and bone, respectively. It was found that different moduli could induce the change of morphology and guide cells to take specific phenotype, as shown in Figure 2-3.

Figure 2-3 Tissue stiffness and its effect on the MSC differentiation. (a) Tissues have a range of elasticity characterized by the elastic modulus, E. (b) MSCs developed different morphology when grown on matrices in the typical range of $\sim \mathrm{E}_{\text {brain, }} \sim \mathrm{E}_{\text {muscle }}$, or $\sim \mathrm{E}_{\text {bone }}$ respectively, scale bar is $20 \mu \mathrm{m}$. (c) $\beta 3$ tubulin (the neuronal cytoskeletal marker), MyoD1 (the muscle transcription factor), and $\mathrm{CBF} \alpha 1$ (the osteoblasts transcription factor) are only expressed on the neurogenic (soft), myogenic, and osteogenic matrices (stiff), respectively; scale bar is $5 \mu \mathrm{m}$. Reprinted (adapted) from Cell, Engler et al., Vol 126, Matrix Elasticity Directs Stem Cell Lineage Specification, 677-689, Copyright (2006), with permission

$$
\text { from Elsevier }{ }^{70} \text {. }
$$

Another interesting phenomenon, which was described as "mechanical memory", was observed in 2010. Gilbert et al. found that pre-cultured muscle stem cells (MuSCs) on soft 
substrates facilitated the in vivo self-renewal of MuSCs; therefore, culture in vitro affects cell behaviors in vivo ${ }^{71}$. Recently, Yang et al. cultured MSCs on a rigid substrate for different duration prior to transfer to a softer substrate, and the correlation between pre-culture duration and osteogenic phenotype was demonstrated as shown in Figure 2-472. A larger proportion of pre-osteogenic transcription factor expression could be found in those cells cultured for the longest time on the stiff substrate prior to seeding on the soft substrate. With 1-day pre-culture, hMSCs demonstrated the fully reversible activation of YAP and RUNX2 (pre-osteogenic transcription factor expression) (Figure 2-4 (b)). With 7-day pre-culture, only a partially reversible activation could be seen (Figure 2-4 (c)). However, with a 10-day pre-culture, the osteogenic phenotype was maintained (Figure 2-4 (d)). Furthermore, the stiffness change was conducted in situ by culturing cells on light-responsive hydrogel, which could be softened from $\sim 10 \mathrm{kPa}$ to $\sim 2 \mathrm{kPa}$ by irradiation at $365 \mathrm{~nm}$ for 6 mins. 


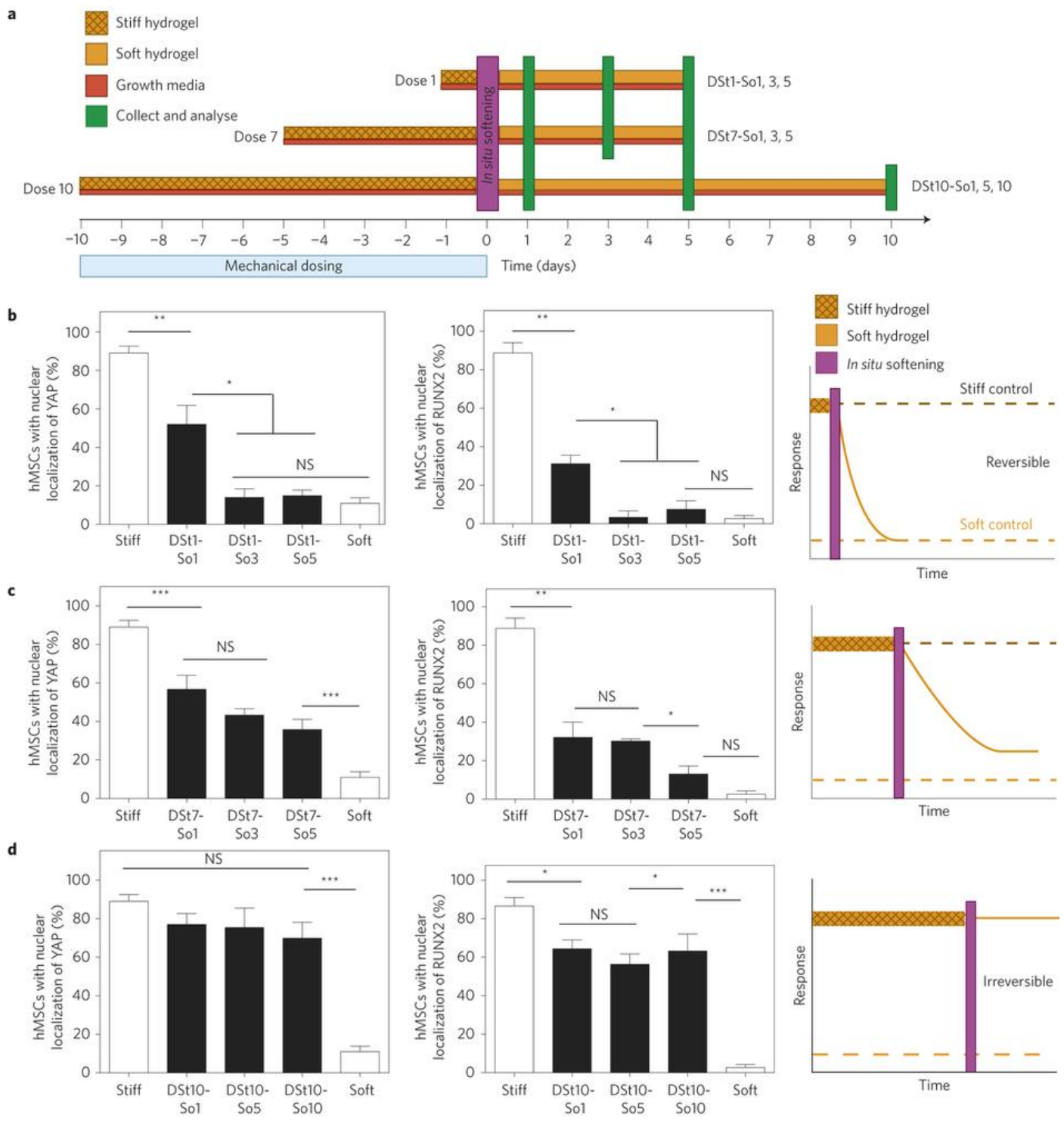

Figure 2-4 Effects of mechanical stimulation by pre-culturing cells on a stiff substrate for (b) 1, (c) 7, and (c) 10 days prior to seeding on soft substrate. (b) With 1-day pre-culture, the activation of YAP and RUNX2 was fully reversible after softening, and (c) it became partially recoverable with the 7-day pre-culture. (d) With the 10-day pre-culture, the activation was irreversible after softening. Reprinted by permission from Macmillan Publishers Ltd: [Nature Materials $]^{72}$, copyright (2014). Additionally, cell spreading and morphology have been found to be relevant to differentiation $^{73}$. Khetan et al. investigated the influence of cell morphology on 
differentiation ${ }^{74}$. Photo-tunable hydrogels, which could be degraded by light exposure, allowed cells to remodel and deform the matrix. MSCs spread in the degradable hydrogels and, therefore, stimulated osteogenesis, whereas MSCs were restricted by crosslinked hydrogels with a circular shape and, therefore, stimulated adipogenesis. A deeper understanding of the correlation between morphology and differentiation came from the study conducted by Huebsch et al. ${ }^{75}$. Murine MSCs were confined to pores in hydrogels with RGD peptide and, therefore, circular morphology was obtained. Interestingly, these cells still showed matrix elasticity-stimulated osteogenesis $(\sim 22 \mathrm{kPa})$, even with circular morphology. The study showed that ligands are very important for cell adhesion and that the subsequent traction generated by matrix reorganization plays a vital role in driving the osteogenic differentiation of $\mathrm{MSCs}^{75}$. 

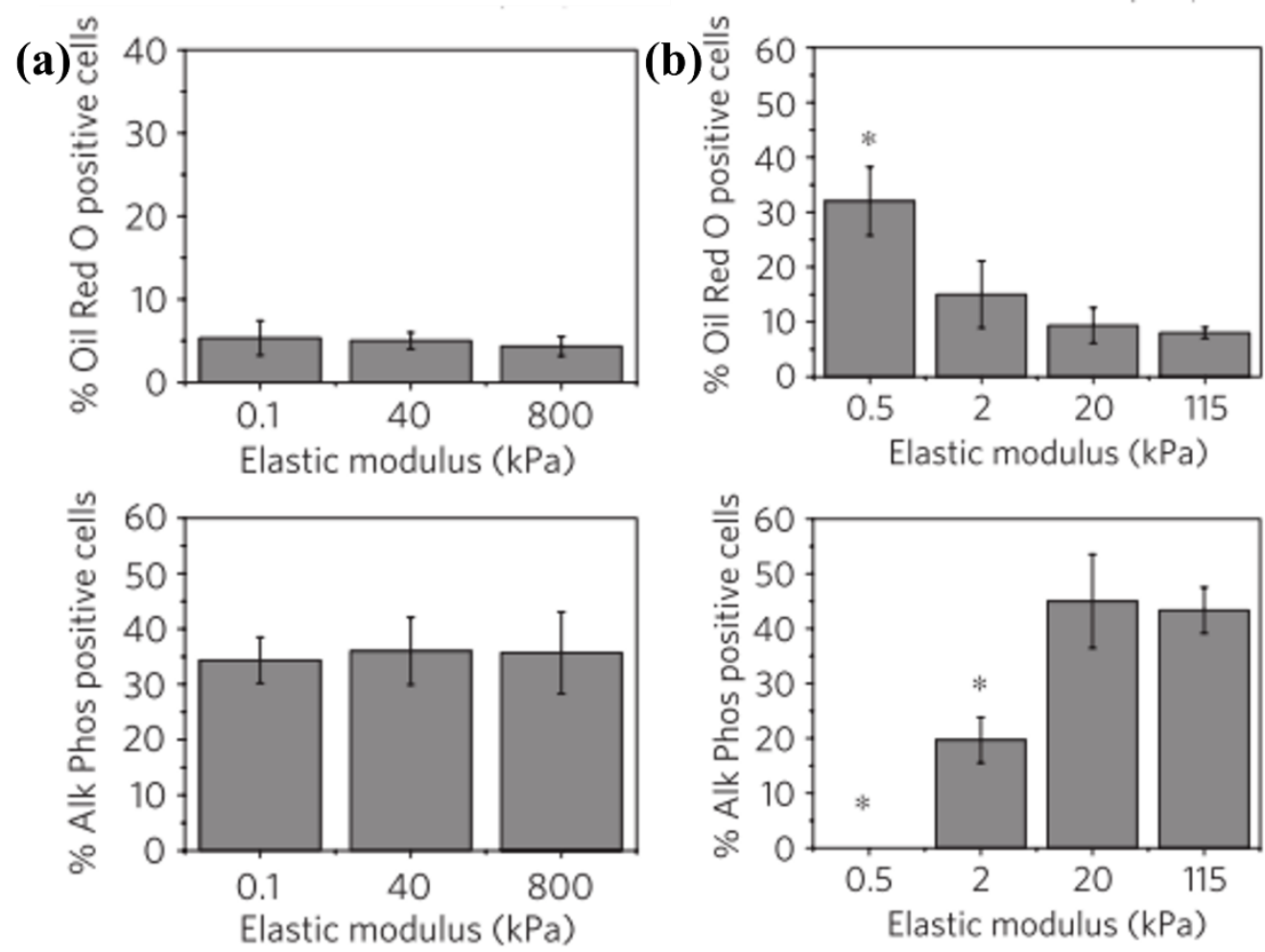

Figure 2-5 Different elastic moduli result in the same trend of cell's phenotype as (Figure

2-3) for PAAm substrates, but no influence on cells cultured on polydimethylsiloxane (PDMS). Reprinted (adapted) by permission from Macmillan Publishers Ltd: [Nature

$$
\text { Materials }]^{76} \text {, Copyright (2012). }
$$

However, the different roles of the bulk stiffness of substrates and the ECM's mechanical properties in cell differentiation need to be clarified. Trappmann et al. reported that different materials with comparable Young's moduli (e.g., PDMS and PAAm gels) could elicit different responses in MSCs, as shown in Figure 2-5 ${ }^{76}$. MSCs cultured on PAAm had the same trend as that Engler et al. demonstrated ${ }^{70}$ (i.e., adipogenic phenotype on the soft surface, osteogenic phenotype on the stiff surface). Conversely, no trend could be seen on PDMS with the same range of moduli. It was demonstrated that the topography (e.g., pore size) of PDMS and PAAm was different; even they had a comparable bulk stiffness. It was 
argued that the different phenotype trend was attributed to the altered adsorption of ECM that changed with the topography. However, Wen et al. argued that differentiation was affected by stiffness regardless of protein tethering ${ }^{77}$. The phenotype was maintained as the pore size of their hydrogel varied.

\subsubsection{Cell Response to Topography}

The interaction between cells and topography was first studied by Harrison in $1911^{78}$. Back in the 1950s, the concept of contact guidance was created when it was found that a change in the appearance of a cell's surroundings altered the morphology of heart fibroblasts $^{79}$. This concept has long been used in the description of conformation to topography $^{80}$. Developments in microelectronics provided a chance to popularize the research in the field through the 1980 s and onward ${ }^{81}$. In vivo, topographical features come from the matrix infrastructure and provide cues for cell behaviors. In vitro, ECM topographical features could alter adhesion, proliferation, cytoskeletal conformation, and gene expression, and related works have been reported by many reseachers ${ }^{17,82-86}$.

Recently, the interactions between cells and their nanoscale environments (i.e., features smaller than a single cell) have attracted a lot of interest ${ }^{87-89}$. The development of novel techniques, such as dip-pen nanolithography (DPN) and electron beam lithography $(\mathrm{EBL})^{90,91}$, has made it possible to pattern features down to $10 \mathrm{~nm}$ in areas with a range from $\mathrm{mm}^{2}$ to $\mathrm{cm}^{2}$, which are large enough for cell tests ${ }^{92}$. For a long time, two main approaches were employed in topographical patterning: highly ordered patterns and random patterns. Ordered patterns, usually fabricated by EBL, show poor cell adhesion. 
Conversely, random patterns can affect the differentiation of MSCs compared with flat controls $^{93}$. Other topography techniques include photolithography ${ }^{94,95}$ and polymer demixing ${ }^{96}$.

Topographical features can also guide axon growth ${ }^{97-100}$. Li et al. found that the depth of topographical features played an important role in neuron growth ${ }^{99}$. Embryonic mouse cortical neurons grew up and down a shallow groove around 2-5 $\mu \mathrm{m}$ but turned at the edge of a higher groove around 22-69 $\mu \mathrm{m}$. Similarly, Mahoney et al. found that the width of microchannels $(20-60 \mu \mathrm{m})$ was important for neurite orientation ${ }^{101}$. Neurites grew parallel to the narrow microchannels $(20-40 \mu \mathrm{m})$, while in wider channels $(40-60 \mu \mathrm{m})$, they grew perpendicular to the channel wall until they reached the channel wall. The contact guidance of axons on a nanopatterned substrate without any chemical treatment was found ${ }^{98}$. Axons preferred to grow on ridge edges and elevations, rather than in grooves, as shown in Figure 2-6. 

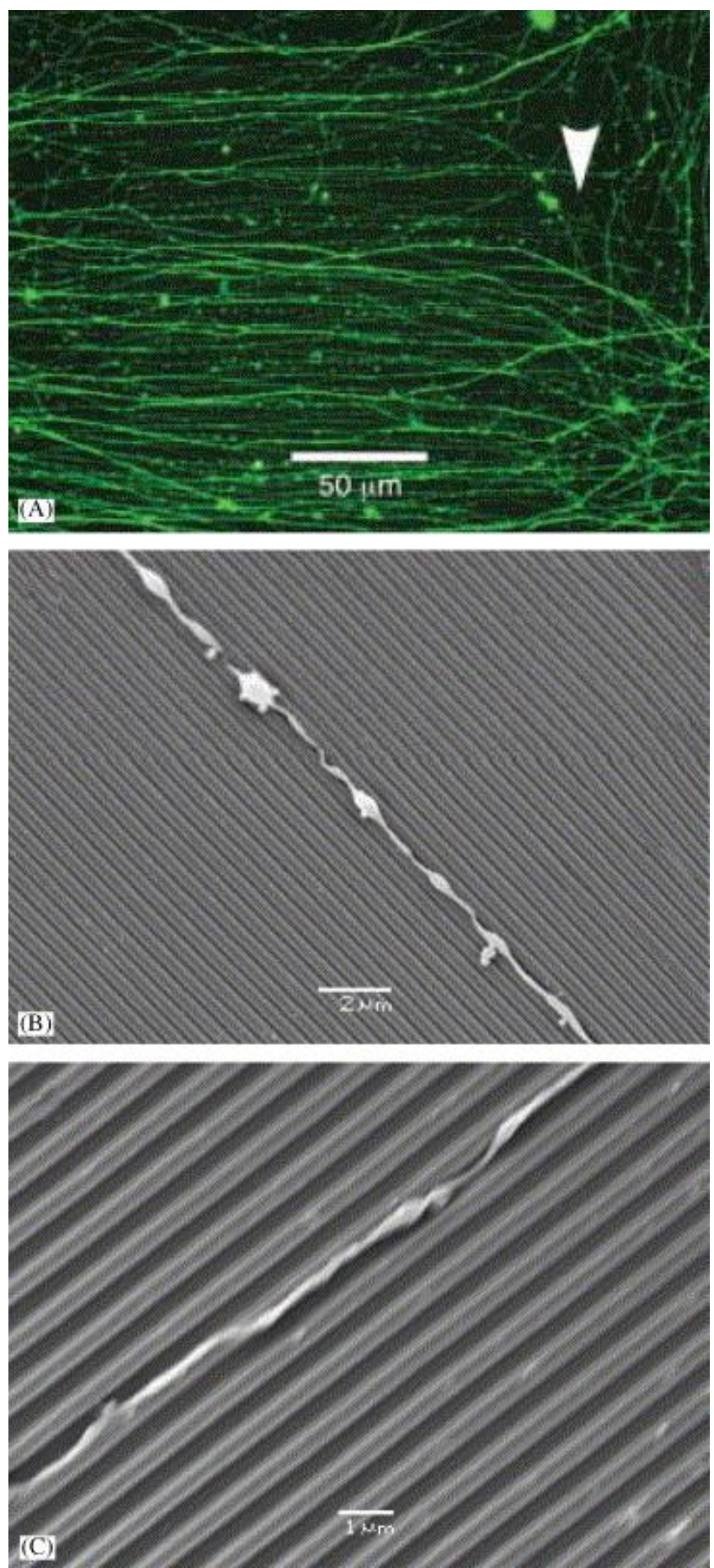

Figure 2-6 (A) Axon growth along a horizontally imprinted pattern (200 nm width, 400 $\mathrm{nm}$ pitch). The arrow marked the border of the pattern. Axon growth on the ridge edges, and not in the grooves. (B): $100 \mathrm{~nm}$ width, $500 \mathrm{~nm}$ pitch; (C): $400 \mathrm{~nm}$ width, $800 \mathrm{~nm}$ pitch.

Reprinted from Biomaterials, Vol. 27, F. Johansson et al., Axonal outgrowth on nano-imprinted patterns, 1251-1258, Copyright (2006), with permission from Elsevier ${ }^{98}$. 
Dalby et al. fabricated different micro-scale patterns, including ordered, controlled-disordered, and random, to investigate the influence of topography on MSCs ${ }^{102}$. While only negligible differentiation was observed on planar, true square patterned, and random substrates, bone differentiation was found on the disordered patterned substrates (Figure 2-7) with close efficiency to that observed following chemical supplement.

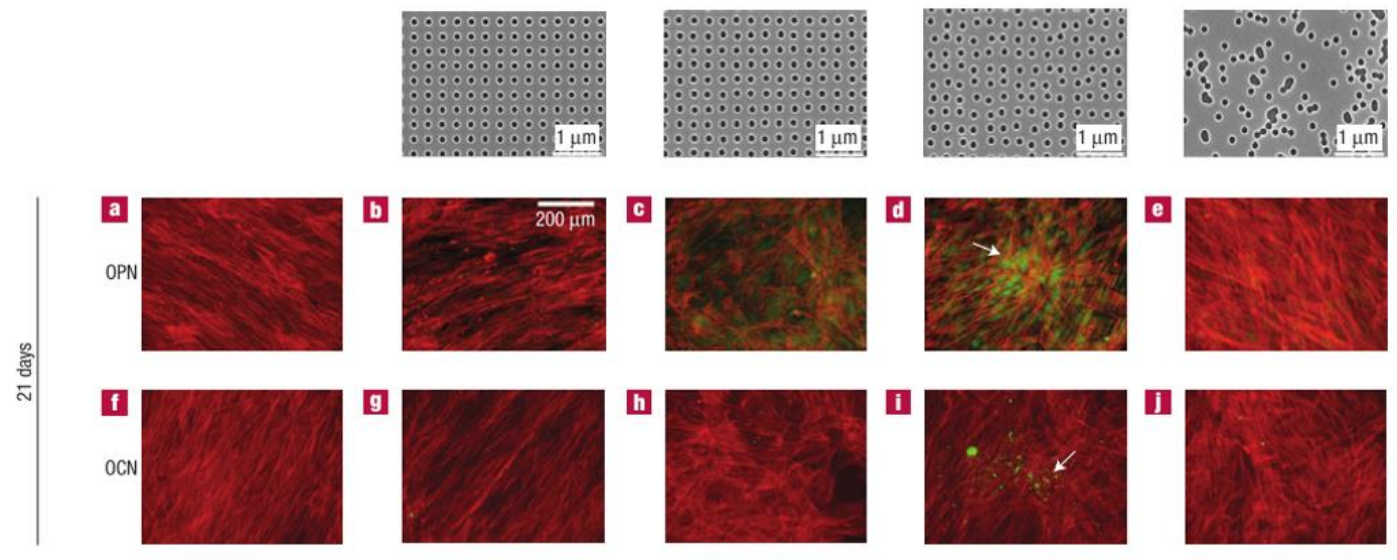

Figure 2-7 EBL patterned structures alter gene expression. Osteogenesis (white arrows) was found to be stimulated in the disordered surface. Reprinted (adapted) by permission from Macmillan Publishers Ltd: [Nature Materials] ${ }^{102}$, Copyright (2007)

The size of the topography could also influence the differentiation of MSCs to a neural lineage. The neuronal marker microtubule-associated protein-2 (MAP2) expressed by MSCs in nanoscale channels increased compared with MSCs in microscale channels, as shown in Figure 2-8 ${ }^{103}$. 


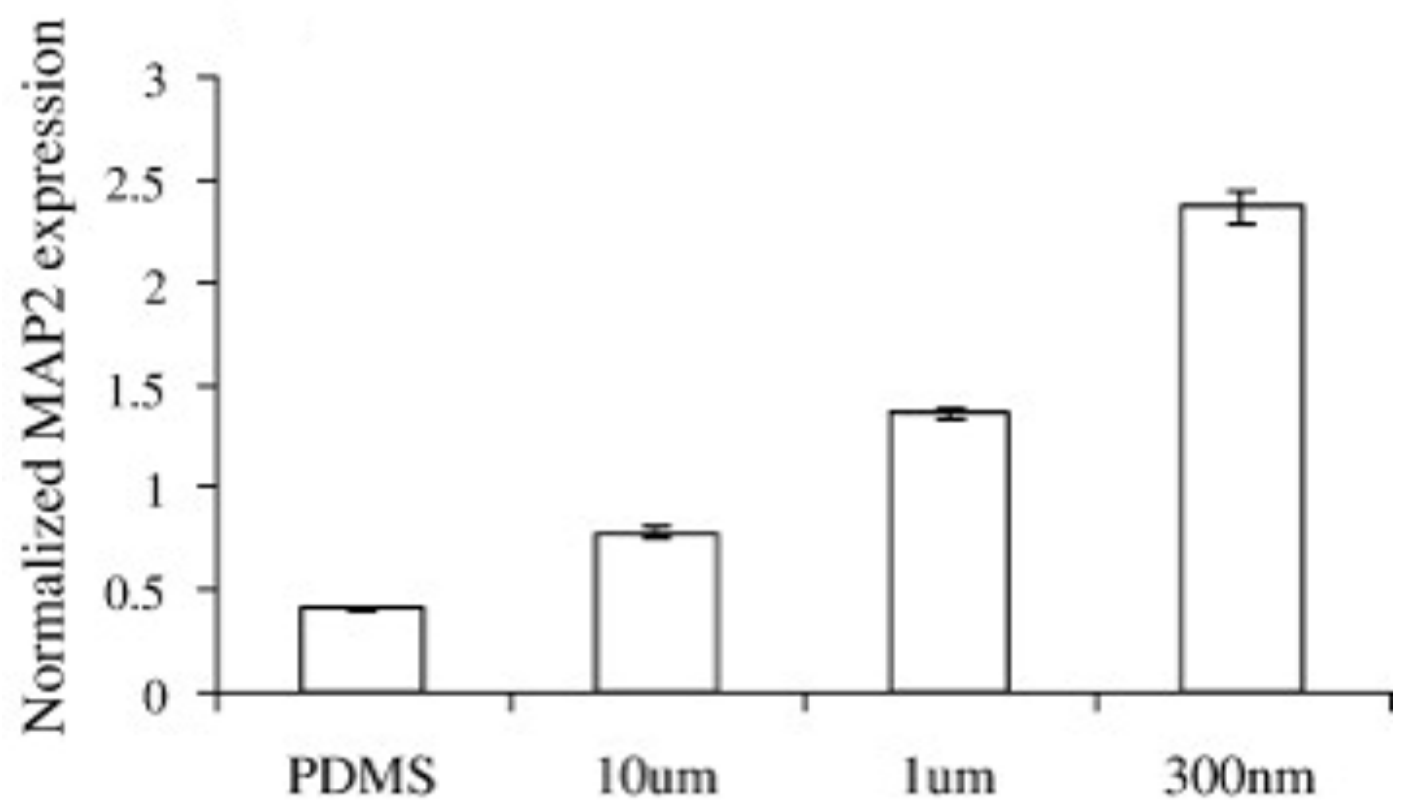

Figure 2-8 Differentiation of MSCs into neural lineage in different substrates, characterized by MAP2 expression. Reprinted (adapted) from Experimental Cell Research, E. Yim et al., Vol. 313, Synthetic nanostructures inducing differentiation of human mesenchymal stem cells into neuronal lineage, 1820-1829, Copyright (2007), with

$$
\text { permission from Elsevier }{ }^{103} \text {. }
$$

Creating a scaffold that could mimic the in vivo 3D architecture of the ECM would be very helpful for tissue engineering. The nanofiber scaffolds with comparable dimensions to the fibrous protein structure of the ECM (50 to $500 \mathrm{~nm}^{104,105}$ ) are believed to be promising materials for cell culture in vitro ${ }^{106}$. It has demonstrated that the rate of neurite growth could be increased when cells were cultured on highly aligned fibers ${ }^{107,108}$. Additionally, the influence of fiber diameter on axon growth has been noted ${ }^{109}$. It was found that filament with diameters in the range of cellular or subcellular size $(5 \mu \mathrm{m}, 30$ $\mu \mathrm{m})$ generates highly aligned and robust neurites outgrowth ${ }^{110}$. Interestingly, nylon fibers embedded in agarose help axon extend along the fiber, while axons in pure agarose could 
not extend due to the lack of adhesive ligands ${ }^{111}$. Based on this idea, 3D microstructures were directly "written" in hydrogel by spatially activating photo-reactive moieties using multi-photon excitation ${ }^{112}$.

\subsubsection{Effects of Mechanical Strain}

The balance of force between cells and the ECM they adhere to is maintained through the actin cytoskeleton of cells. Through specific molecules on cell surfaces, an instantaneous balance change and actin cytoskeleton reorganization could be triggered by the external mechanical strain. This perturbation can change cell shape, trigger some signal pathways, and influence gene expression, and finally, result in cell behaviors change. For example, cyclic tensile strains could stimulate the affected cells to differentiate into smooth muscle cells, because cyclic tensile strain caused by hemodynamic stresses is prevalent in cardiac tissue $^{113}$.

Simplified in vitro models are widely used to investigate the impacts and related mechanisms of mechanical strain in vivo, which usually exhibits complex patterns. With the development of setups, it has become possible to apply a combination of different strains (e.g., translational, rotational, multi-axial strain) to cells to understand the correlation between strain and cell behaviors. The self-renewal of ESCs could be induced by physiologically relevant mechanical strain (biaxial cyclic strain) with maintenance of their pluripotency $(<10 \%$ strain). Above the threshold of $10 \%$ strain, the differentiation was reduced while self-renewal was still promoted ${ }^{114}$. The inhibition of the hESCs differentiation was only observed in cells cultured in conditioned medium, while no 
inhibition was observed in the unconditioned medium. This result indicated a combined effect of mechanical and chemical signaling. Furthermore, TGF $\beta /$ Activin/Nodal signaling may be essential in the regulation of strain-induced differentiation inhibition related to the blocking of $\mathrm{hESC}$ differentiation caused by the upregulation of Smad2/3 phosphorylation $^{115}$.

The potential to maintain the ability of differentiation in a wide range of mechanical microenvironments makes MSCs promising in studies of cellular response to mechanical cues. Different strains have different effects on cells. For example, uniaxial strain was responsible for upregulation of smooth muscle contractile markers' expression and promotion of the differentiation into smooth muscle cells ${ }^{53}$. Conversely, equibiaxial strain induces osteogenesis by activating different kinase pathways ${ }^{116}$. Mechanical strains also promote the expression of some ligament markers in MSCs, such as collagen type I, collagen type III, and tenascin $\mathrm{C}^{117}$. Although numerous studies have been done on the effect of mechanical strain on cell behaviors, these studies still illustrate the enormous possibilities in this area to broaden and deepen our knowledge of stains and their roles in cell behavior regulation.

\subsubsection{Electrical Stimuli on Cells}

ES has become an attractive method of affecting cell behaviors, because endogenous electric fields (EFs) play a vital role in the maintaining of biofunctions of all native organisms, especially in nerves and muscles ${ }^{118,119}$. It is involved not only in well-known action potentials but also in controlling other cell behaviors, including morphology, 
elongation, migration, proliferation, and gene expression ${ }^{120-126}$.

The most basic method of ES involves using a DC voltage, which can be simply generated by batteries ${ }^{127}$. Many other different forms of stimuli have also been employed in in vitro cell cultures, such as monophasic (DC) or biphasic (AC) $)^{120,128}$ sinusoidal ${ }^{122,129,130}$, sawtooth $^{131-133}$, or square wave signals ${ }^{134,135}$ injected in pulses ${ }^{131,132,135}$, pulse bursts ${ }^{122,136}$, or continuously ${ }^{119,120,137}$. Four main techniques are used to deliver ES: direct, capacitive, inductive and combined stimulation. In direct stimulation (coupling), the electrodes are in contact with or inserted into the cell culture ${ }^{128,138,139}$. Although this setup design makes it easy to deliver the ES, the direct contact of the electrodes and cell culture may cause toxicity ${ }^{140,141}$. To avoid these problems, indirect and noninvasive approaches have been used. In capacitive stimulation (coupling), cell culture medium is placed between two parallel plates of metal or carbon with a small gap $(0.5-2 \mathrm{~mm})$ to create a homogeneous electromagnetic field in vertical direction ${ }^{128,131,139,142-146}$. However, its application is limited by the high voltage (e.g., $100 \mathrm{~V}$ ) required to create the electromagnetic field. Inductive stimulation (coupling) utilizes coils placed around the cell culture to create a controlled electromagnetic field. The effect of main parameters (e.g., electric field strength $(\mathrm{V} / \mathrm{mm})$ and current density $\left.\left(\mathrm{A} / \mathrm{m}^{2}\right)\right)$ has been noted ${ }^{42}$.

Galvanotaxis or electrotaxis refers to the migration toward either the cathode or the anode, depending on the particular type of cell, guided by an electric gradient ${ }^{147,148}$. A recent study conducted by Au et al. suggests that the efficiency of the cell alignment of cardiac myocytes and fibroblasts guided by topography is higher than that guided by ES. However, the best result was observed by combining the effects of the two techniques ${ }^{146}$. ES has also 
been utilized to enhance wound healing. For example, a 10-fold increase in the expression of two wound-repair-related cytokines has been demonstrated in the stimulation of cutaneous fibroblasts through conductive scaffolds $^{118}$. PNS and CNS regeneration promoted by ES has been demonstrated in multiple studies ${ }^{56,127,149}$ and already used in some clinical applications ${ }^{150}$. In vitro studies on MSCs and osteoblasts have indicated a certain difference between the effects of the different methods of coupling. For example, ECM deposition, alkaline phosphatase activity, and proliferation could be enhanced by capacitive coupling, whereas differentiation is delayed. When the expression of osteopontin (OPN), BMP-4, and other genes is kept constant, the mRNA levels of BMP-2 and TGF- $\beta 1$ are promoted in some cases ${ }^{120,128,131,137,139,142,151}$.

\subsection{Electrospun Nanofiber and its Applications for Cell Culture}

Electrospinning has been popular for a long period due to an increased interest in nanoscale properties and technologies. This method has been used to fabricate polymer fibers from nanometers to micrometers in diameter ${ }^{152}$. Potential applications of electrospinning include water treatment, fiber-based sensors, and, of course, tissue engineering $^{153,154}$. 


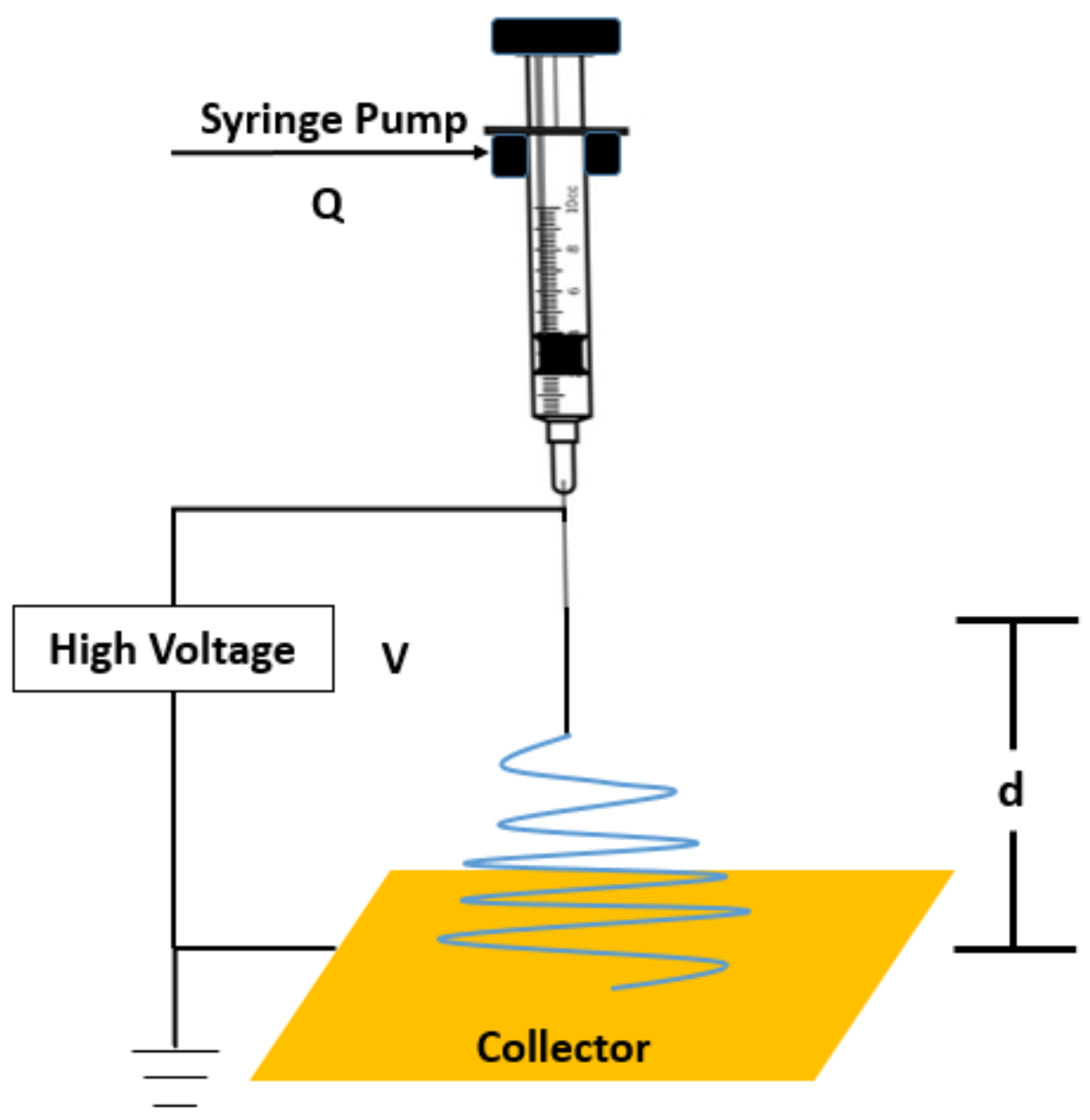

Figure 2-9 Illustration of typical electrospinning setup.

One of the most attractive features of electrospinning is the inexpensive and easy-to-build setup. A typical electrospinning setup consists of a syringe pump, a high-voltage power source, and a collector, as shown in Figure 2-9. A polymer solution is held at a needle tip by surface tension during the electrospinning. The polymer solution is charged by the application of an electric field using the high-voltage power source. The resulting charge repulsion overcomes the surface tension and causes a jet. During jetting, the solvent evaporates and polymer fibers are collected by the collector.

Electrospinning has been widely applied in the tissue engineering area because it provides a simple and low-cost method to produce scaffolds with an interconnected porous 
structures and fiber diameters in the sub-micro range. The highly porous, nonwoven fibrous sheet collected from a typical flat collector is most commonly utilized in tissue engineering because of its high surface area that allows cellular attachment ${ }^{155}$. A number of factors, such as materials, orientation of fibers, the degree of porosity, surface property, etc., should be considered for applications of nanofibers in tissue engineering ${ }^{156}$. The materials utilized in electrospinning include both synthetic materials, natural materials, and the hybrid blends, which can achieve an optimal balance in biocompatible and mechanical properties. The fiber orientation (i.e., aligned and random) and porosity can be optimized for specific applications by varying the parameters of electrospinning. The modification or functionalization of the scaffold could induce other properties, including conductivity and bioactivity, for individual applications.

The most important property of chosen materials is biocompatibility. Toxicity, immune responses, as well as inflammatory, should be controlled. In the subdivision of synthetic materials, both biodegradable and non-biodegradable materials have been studied. The biodegradable ones are more popular because of the elimination of the need for a second operation to remove the implanted scaffold ${ }^{107,157-170}$. In addition, the degradation rate could be optimized to meet the rate of new tissue formation. It has been demonstrated that the blend composition of polymer blends could alter the degradation rate ${ }^{169}$. Changing the proportion of crystalline segment could also affect both the degradation rate and mechanical properties ${ }^{162}$. Besides the degradation rate of the scaffolds, the mechanical properties also matter ${ }^{170}$. Although biodegradable materials have obvious advantages, some researchers in the field of tissue engineering investigated non-degradable materials 
and found they were able to support cell culture in vitro ${ }^{171,172}$. To mimic the native ECM, natural materials, such as chitosan, fibrinogen ${ }^{173}$, $\operatorname{chitin}^{174}$, hyaluronic acid $(\mathrm{HA})^{175}$, collagen ${ }^{176,177}$, and gelatin ${ }^{178,179}$ have been utilized for electrospinning in tissue engineering. However, the lack of proper mechanical properties and difficulties in electrospinning of natural materials led to the utilization of hybrid materials consisting of both synthetic and natural materials ${ }^{175,180-189}$. The addition of synthetic materials usually improved the mechanical properties, such as Young's modulus and tensile strength, while natural materials provided the bioactive sites to help cell adhesion and growth.

By using different collectors, different orientations of fibers could be obtained. For example, a stationary collector is usually used to form random fibers, while a rotating collector is for aligned fibers. The anisotropy degree within electrospun fibers has been shown to greatly influence both the mechanical properties and cell behaviors, like proliferation, adhesion, alignment, and differentiation. A lot of studies have proven that developing an aligned fibrous mat could be more desirable in certain applications (e.g., to replace a highly oriented tissue $)^{107,159,161,165,168,178,190,191}$. The aligned fibrous mat could align the cytoskeleton of cells parallel to the fibers and the adhesion and proliferation could be improved compared to a random mat ${ }^{168}$. In peripheral nerve generation, it has been demonstrated that axonal outgrowth and glial cell migration could be directed by aligned scaffolds ${ }^{159}$.

Porosity and pore size also play important roles in helping cell migration and infiltration. By choosing different polymer/solvent systems and various processing parameters, varied pore sizes can be achieved. The effect of fiber width and fiber density on the mean pore 
radius has been studied by using a theoretical model ${ }^{192}$. Researchers have found that the mean pore size increases with increasing fiber diameter when the porosity and areal density were given, which led to an interesting paradox. People are trying to develop smaller diameters for an increasing surface area; however, it will result in a small pore size, which will limit cell infiltration and migration. Thus, 2D nanofibers usually behave as $2 \mathrm{D}$ meshes rather than as 3D scaffolds. Although the layer-by-layer mesh stacks have been developed, they still reduce the pore size due to the high areal density. Of course, the small pore size can actually become an advantage when cell infiltration is undesirable. These nanofibers could be utilized for barrier applications, like skin. On the other hand, large pores sizes have been developed for applications in 3D scaffolds ${ }^{170,193,194}$.

Another important way to engineer nanofibers is to functionalize the surface of nanofibrous scaffolds. A number of bioactive groups have been tested, such as $\mathrm{RGD}^{195}$, gelatin $^{196}$, perlecan ${ }^{197}$, and acrylic acid ${ }^{160}$. For example, RGD can enhance the attachment, spreading and proliferation of $3 \mathrm{~T} 3$ fibroblasts ${ }^{195}$. Some other treatment, like PPy coating, could induce the conductivity into the nanofibers, which could be useful for applying ES to cells ${ }^{10,198}$.

Electrospinning has been found to be highly promising in various applications in tissue engineering including, vasculature ${ }^{168,180,199-203}$, bone ${ }^{165,181,183,204-206}$, neural $1^{107,159}$ and tendon/ligament ${ }^{207,208}$ applications. 


\section{Chapter 3. Long Term Effects of Substrate Stiffness on the Development of hMSC Mechanical Properties}

\subsection{Significance and Novelty}

Although the influence of substrate stiffness on cellular mechanical properties has been studied, previously, the researchers paid more attention to the direct influence of the substrates. However, the combined effect of the substrate stiffness and a long-term culture time, i.e., two weeks, on cells' mechanical properties is still unclear. To the best of the author's knowledge, the study of the combined effect of both substrate stiffness and a long-term culture time ( 2 weeks) on cellular moduli characterized with micropipette aspiration (MA) is new. Besides a positive correlation of cell moduli and substrate stiffness which is similar to previous studies, our results showed an unreported non-monotonic trend of cell moduli as a function of time. This time-dependent change of cellular moduli showed the cellular moduli in different substrates increased over the first 11 days and followed with the moduli drop from day 11 to day 14 . The moduli increase could be explained by the cytoskeletal coarsening caused stiffening in cells, which was supported by both the fluorescent images in our study and the previous studies. The moduli drop could be attributed to osteogenic differentiation caused softening. Although further investigation is needed to enhance this explanation, this work offered the new insight into the relation between cellular moduli of MSCs and substrates/culture time. 


\subsection{Introduction}

MSCs are multipotent stem cells mainly isolated from bone marrow. Other sources include liver, amniotic fluid, and placenta ${ }^{209}$. The significance of studying MSCs lies in their ability to differentiate into different cell lineages ${ }^{4}$ as well as their promising potential to be applied to cell and gene therapy for serious diseases like heart failure or hematological pathologies ${ }^{209}$.

Cellular behaviors of MSCs including locomotion, proliferation, and differentiation depend a lot on their growing microenvironment ${ }^{51-54}$. Because it is necessary for tissue cells to adhere to and grow on solid surfaces ${ }^{60}$, the influence of substrate properties, like stiffness, on cells has been extensively studied ${ }^{52,70,210-213}$. It has been found that the human Mesenchymal Stem Cells (hMSCs) became stiffer when cultured on stiffer substrates ${ }^{52}$. With or without induction factors, it has been suggested that the stiffness of substrates could direct the lineage specification of $\mathrm{hMSCs}^{70,214}$ and the viscoelasticity of hMSCs changed monotonically with time during differentiation ${ }^{215}$. It was also shown that MSCs grew on soft substrates tended to be neurogenic while on stiffer substrate tended to be myogenic or even osteogenic ${ }^{70}$. The culture time on substrates with different stiffness could even influence the following differentiation behavior of the MSCs. MSCs could easily differentiate into different lineages when induction factor was added after a short time period of culture on the substrates while they became difficult to be induced to other lineages except the substrates-defined lineage after a long time period of culture on the substrates $^{70}$. For example, if the MSCs were cultured on the soft neurogenic matrices for three weeks, they could only be induced to neurons even if myogenic or osteogenic factors 
were added into the media. Nonetheless, a good method to distinguish the potential of stem cells to differentiate into specific lineages was yet to be established ${ }^{216}$. Recent studies confirmed the feasibility of correlating differentiation behavior of stem cells with their mechanical properties, for example, osteogenesis was positively correlated with the elastic as well as viscous properties of the cells ${ }^{215,216}$. Therefore, cell mechanical property measurement could serve as an easy and effective characterization of cellular behaviors.

Micropipette aspiration (MA) has been widely applied to measure cell mechanical properties $^{215,217-222}$, which is based on the detection of the cellular deformation under a given external pressure. Cells and the external environment they adhered to are connected through cellular cytoskeleton. From either extracellular matrix or neighbors, cells could sense the external mechanical strains that guide cell behaviors like migration, differentiation and so on. The cell deformation is driven by the polymerization and depolymerization of actin filaments and microtubules together with molecular motors. The internal or external mechanical forces could affect several classes of regulatory proteins involved in the cellular architecture, thus, the local organization of the cytoskeletal network $^{223}$. For example, recent studies have demonstrated that the contractile force of cellular actin-myosin cytoskeleton, which was determined mainly by cells' sensing of the surrounding extracellular matrix, could affect cytoskeletal organization ${ }^{60}$. Compared with other commonly used methods, such as atomic force microscopy (AFM) ${ }^{224,225}$ and optical $\operatorname{traps}^{226,227}$, MA method has the ability to generate large forces and measure the global mechanical properties of the investigated cell. Depending on the cellular behavior in the micropipettes, various models have been developed to extract cell mechanical 
modulus $^{218,228}$. For hMSCs, both elastic and viscoelastic solid models have been used in the previous studies ${ }^{215,221,222}$.

However, the long-term relationship between substrate stiffness and mechanical properties of cells has not been well studied. The objective of this study was to investigate the long-term influence of substrate stiffness on the mechanical properties of hMSCs. Instead of the commonly used substrate, such as polyacrylamide ${ }^{70,210}$, polydimethylsiloxane (PDMS) substrate was adopted for the easy preparation procedure and easy stiffness control, which only requires modification of the ratio between the base material and the curing reagent. The hMSCs were cultured on PDMS substrate without adding any induction factor for a total duration of two weeks and measured at six time-points - day 0 , $1,5,7,11$, and 14 . Three replicates were obtained for each sample and the data were analyzed using two-way analyses of variance (ANOVA) so that not only the influence of substrate stiffness but also the culture time could be evaluated.

Because change in cell stiffness is expected to have a major impact on cell mechanosensitivity and mechanotransduction, for example, the cellular mechanical properties of stem cells could reflect the differentiation potential ${ }^{215,216}$; the moduli change with different substrate stiffness and culture time probably relate to the different stages of differentiation. The results, cellular moduli change over different substrate stiffness and culture time, revealed in this study could give a new angle to investigate the cellular behaviors, for example, differentiation and attachment. For example, it could provide a new index to determine an optimal time point and substrate stiffness of specific differentiation induction in future. 


\subsection{Materials and Methods}

\subsubsection{Fabrication of the PDMS Substrates with Different Stiffness}

Instead of the commonly used substrate, such as polyacrylamide ${ }^{70,210}$, polydimethylsiloxane (PDMS) substrate was adopted for the easy preparation procedure and easy stiffness control, which only requires modification of the ratio between the base material and the curing reagent.

PDMS (Sylgard 184, Dow Corning, USA) substrates with different stiffness were prepared by mixing the base and the curing agent in varying weight ratios. Higher ratios result into lower moduli due to lower crosslink densities ${ }^{229}$. Same amount of PDMS mixtures with base-to-reagent ratios of 5:1 (PDMS5), 10:1 (PDMS10), 20:1 (PDMS20), and 30:1 (PDMS30) were poured into 24-well plates, and a $1 \mathrm{~mm}$ thick substrate was formed in each well. The plates were then kept in a $60^{\circ} \mathrm{C}$ oven for $2 \mathrm{~h}$ for PDMS solidification after degassing. The cured PDMS substrates were thoroughly washed in DI water and $70 \%$ ethanol solution.

As cell attachment is limited by the hydrophobic nature of PDMS ${ }^{230,231}$, poly-L-lysine (PLL, Sigma, Singapore) coating was conducted. To improve the hydrophilicity, further remove residuals, and improve PLL coating, we conducted an oxygen plasma treatment (MARCH, PX-500). The $\mathrm{O}_{2} \%$ is $50 \%$, and the pressure is 75 mTorr. The substrates were exposed for $5 \mathrm{~min}$. The treated surfaces were immersed in sterile DI water immediately after the plasma to prolong the surface hydrophilicity ${ }^{232}$. DI water was removed before 
PLL treatment. The substrates were immersed in PLL solution $(0.01 \% \mathrm{w} / \mathrm{v})$ for $30 \mathrm{mins}$ and washed with sterile DI water before cell seeding. The polycation nature of PLL leads to its electrostatic attraction onto a negatively charged plasma-oxidized PDMS surface, which resulted in a stable coating of PLL up to 12 weeks $^{233}$. The images of adhesion of hMSCs on the PDMS substrates with or without PLL treatment were shown in Figure 3-4. It indicated PLL treatment could obviously improve cell attachment. Thus, the PLL coating is mainly to promote cell adhesion ${ }^{234-237}$. Additionally, the cells cultured on treated PDMS substrates for 5 days and 14 days showed similar morphology as shown in Figure 3-5, which indicated a stable PLL coating. However, some previous studies suggested that poly-L-lysine could promote the chemically-inducted chondrogenic and osteogenic differentiation of $\mathrm{hMSCs}^{238,239}$, so the cell differentiation was characterized here. The moduli of different PDMS materials were characterized using an Instron machine.

\subsection{2 hMSC Recovery, Expansion, and Seeding}

The formulation of culture medium followed the previous study ${ }^{214,231}$. Briefly, the culture medium was prepared by adding 10\% fetal bovine serum (FBS) (Life Technologies, Singapore.) and the mixture of penicillin/streptomycin (Life Technologies, Singapore) to Dulbecco's modified Eagle's medium (DMEM) (Life Technologies, Singapore).

hMSCs derived from human bone marrow were obtained from Life Technologies, Singapore. The frozen vial of hMSCs was rapidly thawed in a $37^{\circ} \mathrm{C}$ water bath until a small amount of ice remained. The entire contents of the vial were pipetted into a $15-\mathrm{mL}$ conical tube. We carefully added $5-10 \mathrm{~mL}$ of pre-warmed medium to the conical tube at a 
rate of approximately 3 to 5 drops per 5 seconds and gently swirled after every addition. The cells were centrifuged at $200 \times \mathrm{g}$ for $5 \mathrm{mins}$ at room temperature. After removing the supernatant, the cells were resuspended in pre-warmed medium and seeded in a culture flask (ThermoFisher Scientific, Singapore) with $5 \times 10^{3}$ cells $/ \mathrm{cm}^{2}$. The culture flasks were used directly without PLL treatment.

To expand and subculture hMSCs, we removed medium from the culture flask and gently rinsed cells with sterile PBS once a confluence of $80 \%-90 \%$ was achieved within 2-4 days. $3 \mathrm{~mL}$ pre-warmed $0.25 \%$ Trypsin (Life Technologies, Singapore) was added to each T75 flask. Trypsin is a proteolytic enzyme commonly used to dissociate adherent cells from the vessel in which they were cultured. It can "digest" the proteins that facilitate adhesion to the container and between cells. The morphological sequence of events occurring during the initial rounding-up of cells incubated in trypsin has been well documented ${ }^{240}$. Briefly, after treatment with trypsin for several minutes, the cells began to round-up, leaving behind long retraction fibrils. The retraction fibrils, attached by their distal end to the substrate, became fewer and fewer, probably because they gradually lose their attachment and retract. After a certain period, the cells detached from the surface and changed to a spherical structure. The cells were incubated at $37^{\circ} \mathrm{C}, 5 \% \mathrm{CO}_{2}$ incubator for 4 mins before pre-warmed culture medium was added. We then transferred the contents to a conical tube and centrifuged cells at $400 \times \mathrm{g}$ for 5 mins. The supernatant was then aspirated. The cells were resuspended in the fresh pre-warmed culture medium and counted. Finally, the cells were seeded in a new flask with a density of $5 \times 10^{3}$ cells $/ \mathrm{cm}^{2}$.

Cells of passage 2 to 4 were used in our experiment. After detaching from the culture flask 
using $0.25 \%$ Trypsin (Life Technologies, Singapore) for less than four minutes, hMSCs were seeded onto the prepared PDMS substrates with a density of around 5,000 cells per well in 24-well plates. Inoculated cells were kept in the culture medium, which was changed twice a week, before micropipette aspiration. Before micropipette aspiration, cells were detached with $0.25 \%$ Trypsin (Life Technologies, Singapore) treatment for less than 4 mins, and then immediately placed in the culture medium for aspiration. Because previous study concluded a trypsin treatment less than 4 mins would not affect the mechanical properties ${ }^{241}$.

\subsubsection{Fluorescent Staining and Imaging}

Cells were fixed in the $10 \%$ neutral buffered formalin solution (Sigma, Singapore) for 20 min and treated with $0.1 \%$ Triton X-100 (Sigma, Singapore) for 5 min followed by two times PBS wash. F-actin filaments were stained by 1-hour incubation with rhodamine-conjugated phalloidin (Life Technologies, Singapore) at 1:500 dilution at room temperature. To stain nuclei, each well was incubated with DAPI (Life Technologies, Singapore) at 1:1000 dilution for 5 min. Images were taken with an inverted fluorescent microscope.

\subsubsection{Osteogenic Specification}

To verify whether the MSCs had differentiated to osteoblast, one osteoblast biomarker, anti-RUNX2 antibody, was used. Cells were fixed in the $10 \%$ neutral buffered formalin solution (Sigma, Singapore) for $20 \mathrm{~min}$ and treated with $0.1 \%$ Triton X-100 (Sigma, 
Singapore) for 5 min followed by PBS wash for two times. Then cells were incubated with $1 \%$ BSA blocking buffer for 1 hour at room temperature. The cells were then incubated with primary antibody, anti-RUNX2 antibody (Millipore, Singapore), in 1:1000 dilution for overnight at $4{ }^{\circ} \mathrm{C}$ followed by three times PBS wash. Cells were then incubated with Alexa Fluor ${ }^{\circledR} 488$ secondary antibody (Millipore, Singapore) at 1:1000 dilution at room temperature for 1 hour followed by DAPI staining.

The osteogenic lineage formation was also demonstrated by Alizarin Red staining, whereby the presence of brick-red nodules revealed the mineralization during osteogenic differentiation. Fixed samples were washed by DI water for three times and incubated in Alizarin Red for 5 mins at room temperature. After removing Alizarin red and washing with DI water, images were taken.

To prepare the Positive Control Groups, we cultured cells on different PDMS substrates with the same density before osteogenesis. Induction of osteogenic differentiation in MSCs was initiated by introducing the osteogenic medium, comprising low glucose DMEM supplemented with $10 \%$ FBS, $50 \mu \mathrm{g} / \mathrm{ml}$ ascorbic acid, $1 \mathrm{mM}$ sodium pyruvate, $100 \mathrm{U} / 100 \mu \mathrm{g}$ penicillin/streptomycin, $1 \times$ glutamax, $10 \mathrm{mM} \beta$-glycerophosphate, and $10^{-7}$ M dexamethasone ${ }^{230}$, on day 1 (the day after seeding). The cells were then cultured in a humidified atmosphere with $5 \% \mathrm{CO}_{2}$, and the differentiation medium was changed twice per week.

\subsubsection{Micropipette Aspiration}

The micropipette aspiration system is illustrated in Figure 3-1. A micromanipulator was 
used to move a micropipette with a sharp tip close to a cell. A microinjector was used to generate the pressure applied to the cell, which would be measured using a pressure transducer, a demodulator, and a digital multimeter. A microscope and a computer were used to locate the cells and record the images during the aspiration. Micropipettes of inner diameters from $8 \mu \mathrm{m}$ to $12 \mu \mathrm{m}$, which are around 0.2 to 0.8 times of cell diameters, were made with a pipette puller (Sutter Instrument, USA).

The calibration was conducted as instructed in the manual before the experiment. Firstly, the air was completely removed from the system with DI water. The micropipette tip was placed into a $1000 \mathrm{ml}$ measuring cylinder. The voltages were recorded as well as the height of water added into the measuring cylinder. The voltage obtained was a function of the height of water due to the hydrostatic pressure. 4 runs were performed and the linear relation of $\mathrm{P}$ and $\mathrm{V}$ were calculated.

At the beginning of an experiment, the whole system was washed with deionized (DI) water and filled with PBS solution successively using the syringe to remove air bubbles trapped in the system. After that, the cell of interest was placed in the middle of the view field under the microscope by moving the microscope stage. Then the micropipette was moved close to the cell using the micromanipulator and an equilibrium pressure, under which the cell was neither sucked in nor pushed away, was achieved through the microinjector (Narishige, Japan) before an aspiration pressure was applied to deform the cell. The applied pressure was measured by a pressure transducer (Validyne Engineering, USA), converted to voltage values by a demodulator (Validyne Engineering, USA). The final reading was then obtained from a digital multimeter (Fluke, USA). A total of 30 
images were taken during the around 300s aspiration process using an inverted microscope (Nikon, Japan) with a camera (Tucsen, China). Open source software (ImageJ by NIH, USA) was used for the image processing to get the aspiration length data.

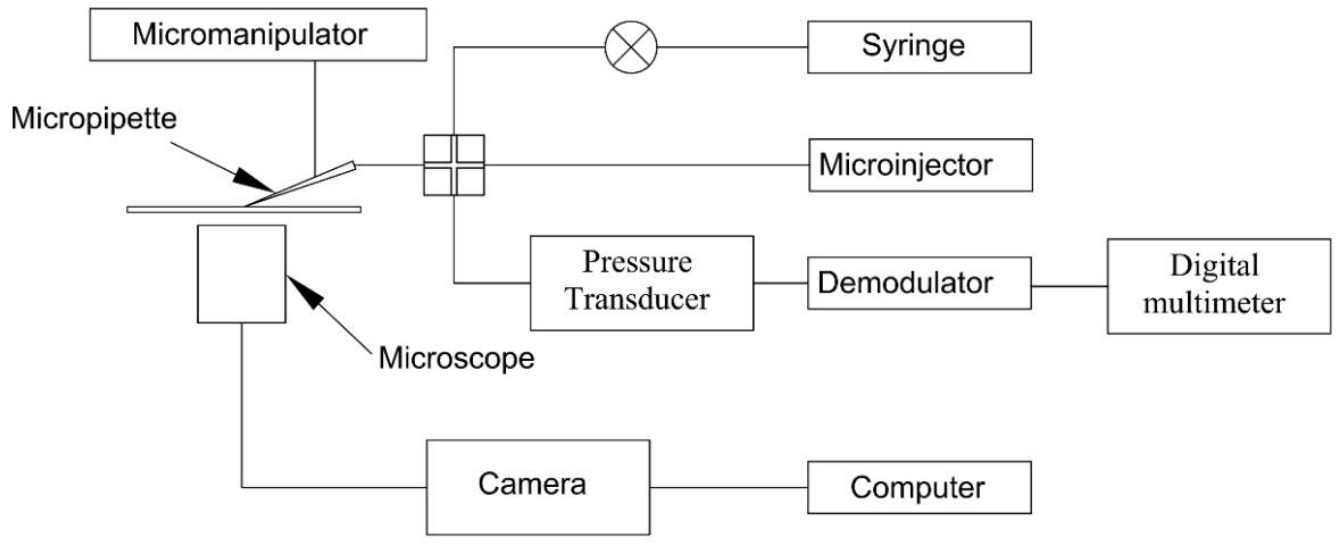

Figure 3-1 Micropipette aspiration system illustration.

\subsubsection{Theoretical Modelling}

Although we recognize that the hMSC is morphologically heterogeneous, the homogeneous solid model was fitted to the viscoelastic properties of hMSCs excellently both in previous studies and our report ${ }^{221,222}$. The hMSCs under our experimental conditions represented a typical viscoelastic solid creep behavior characterized by an initial jump in deformation in response to a step aspiration pressure followed by an asymptotic creep behavior until equilibrium (see details in 3.4.4). Although the linear elastic model is a simplified model ignoring the time factor, it was demonstrated this model could characterize the elastic properties of solid-like cells ${ }^{218,228,242}$. The linear elastic model and the linear viscoelastic model, in which the cell is assumed to be homogeneous ${ }^{228}$, were used to extract the mechanical properties of hMSCs. 
The linear elastic model is a simplification of the viscoelastic model through ignoring the time factor. In this model, the cell is approximated as an incompressible elastic half-space ${ }^{218,228,243}$, i.e., the local radius of the cell surface is relatively large compared to the pipette radius $R_{p}$. The elastic modulus $E$ of the cell is calculated as $^{243}$ :

$$
E=\Phi\left(\frac{3 R_{p}}{2 \pi}\right)\left(\frac{\Delta P}{L_{a}}\right)
$$

where $L_{a}$ is the equilibrium aspiration length, $\Delta P$ is the applied aspiration pressure, and $\Phi$ is a constant related to the ratio of the pipette wall thickness to the pipette radius, which is chosen to be 2.0 in this study based on the punch model ${ }^{243}$.

In the viscoelastic model, three parameters (Figure 3-2) were obtained through evaluating the real-time influence of $\Delta P$ on the aspiration length $(L(t))$ of the selected cell. The relationship between $L(t)$ and the time passed since the pressure is applied could be fitted using the following equation ${ }^{244}$ :

$$
L(t)=\frac{\Phi R_{p} \Delta P}{\pi k_{1}}\left[1-\frac{k_{2}}{k_{1}+k_{2}} e^{-t / \tau}\right]
$$

where $\Phi, R_{p}$, and $\Delta P$ have the same definition as in the elastic model (Equation 3-1). $k_{1}$ and $k_{2}$ are the elastic constants, as shown in Figure 3-2. In this model, a viscous material is modeled as a spring and a dashpot in series with each other, both of which are in parallel with a lone spring. Under a constant stress, the modeled material will instantaneously deform to some strain, which is the elastic portion of the strain, and after that it will continue to deform and asymptotically approach a steady-state strain. This last portion is the viscous part of the strain. $\tau$ is the exponential time constant. The apparent viscosity $\mu$ is given by ${ }^{244}$ : 


$$
\mu=\frac{\tau k_{1} k_{2}}{\left(k_{1}+k_{2}\right)}
$$

Equation 3-3

$k_{1}$ and $k_{2}$ are both needed to calculate the standard elasticity coefficients:

$$
\begin{array}{cc}
E_{0}=\frac{1}{2}\left(k_{1}+k_{2}\right) & \text { Equation 3-4 } \\
E_{\text {eqm }}=\frac{1}{2} k_{1} & \text { Equation 3-5 }
\end{array}
$$

where $E_{0}$, the instantaneous Young's modulus, is defined to reflect the initial elastic response at the very beginning of aspiration., and $E_{\text {eqm }}$ is the equilibrium Young's modulus determined at the point that suction length was no longer increased 221,222 .

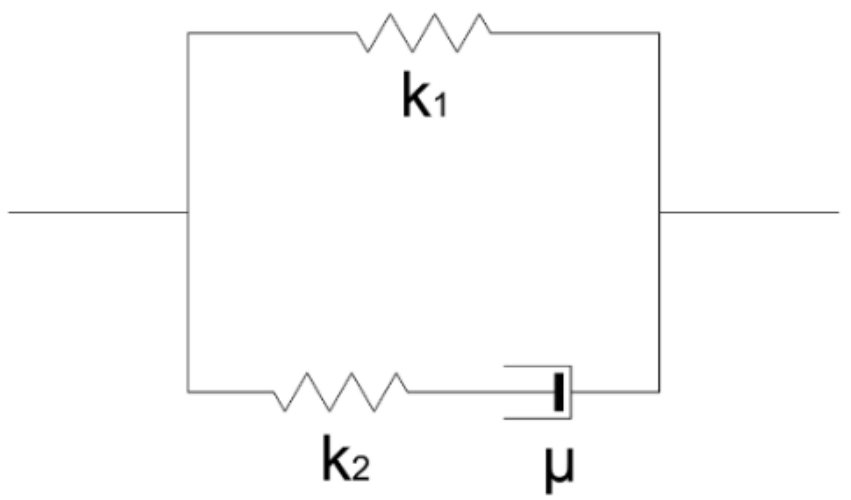

Figure 3-2 Illustration of the viscoelastic model, where $k_{1}$ and $k_{2}$ are the elastic constants of two springs; $\mu$ is the viscosity of a dashpot.

\subsubsection{Statistical Analysis}

The elasticity data of PDMS substrates were analysed using one-way analyses of variance (ANOVA) while two-way ANOVA method was applied for the elastic and viscoelastic data of cells, with PDMS stiffness and culture time as two independent variables $(n=3)$. Tukey's post-hoc tests were performed when significance was indicated in ANOVA results. The F value, F (DF1, DF2), was calculated by the software. DF1 is the degree of freedom 
for main factors $(4-1=3$ for stiffness or $5-1=4$ culture day) or interaction $((4-1) \times(5-1)=12$ for stiffness $\times$ culture time); DF2 represents the degree of freedom for the residual $((4 \times 5 \times 3-1)-3-4-12=40)$. The $p$ values calculated from $\mathrm{F}$ and the two degrees of freedom indicate the significance of the main factors and interactions. Significance was determined at $p<0.05$ and a trend towards significance was determined at $p<0.1$. All values were reported as mean \pm standard error of the mean (SEM) or standard deviation as indicated. The statistical analysis was conducted with GraphPad Prism 6.05.

\subsection{Results}

\subsubsection{Stiffness of Different PDMS substrates}

The Young's moduli of different PDMS measured using Instron extension test showed a clear trend that with a higher base to curing agent ratios the PDMS became softer (Figure 3-3). And the Young's moduli of PDMS substrates fall into the range from $0.06 \mathrm{MPa}$ to 1.2 $\mathrm{MPa}$, which could cover the stiffness range from muscle to precalcified bone ${ }^{245}$. 


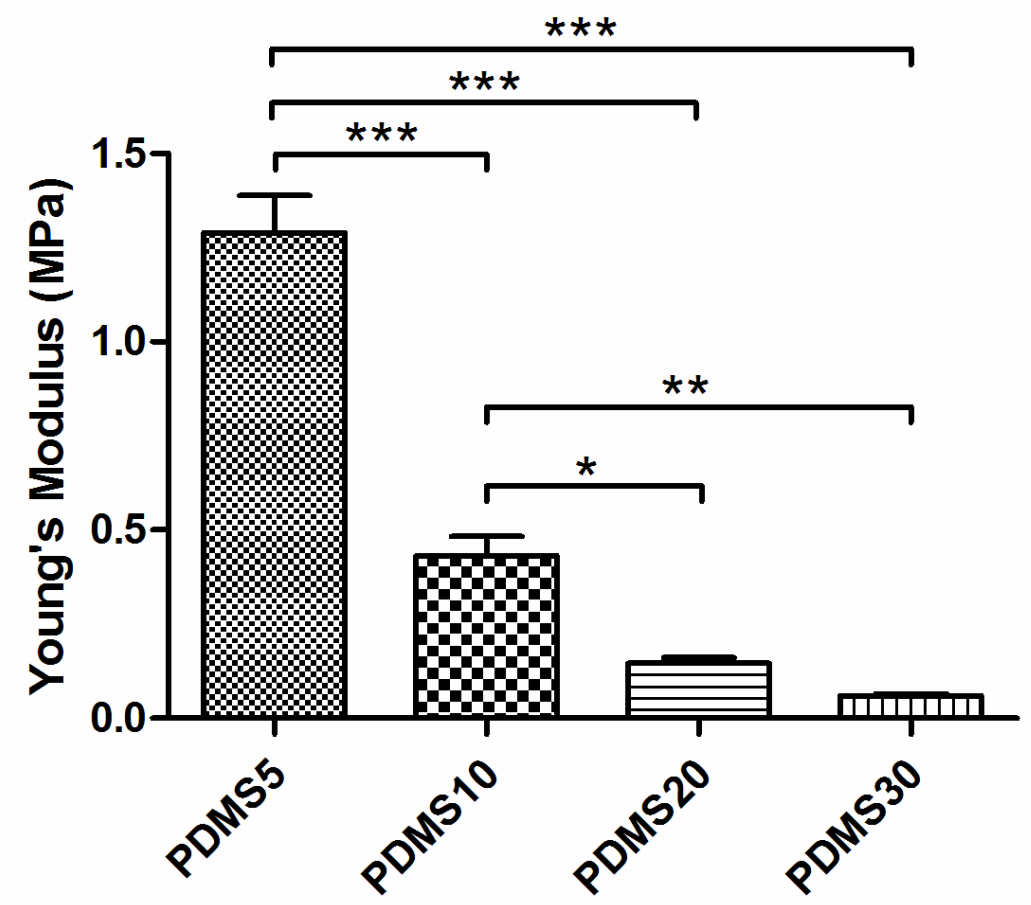

Figure 3-3 Young's modulus of PDMS measured by Instron machine $(*$ indicates significant difference between the two groups with $p<0.05, * *$ for $p<0.01$, and $* * *$ for $p<0.001)$

\subsubsection{Morphology of hMSCs on PDMS}

The adhesion of hMSCs cultured on bare PDMS (sterilized by $70 \%$ ethanol, no plasma and PLL treatment) and PLL-treated PDMS were compared in Figure 3-4. hMSCs were seeded on the PDMS10 substrate with the same concentration $(5,000$ cell/well in 24 -well plates). After one day, the cells were fixed and stained to see the difference in morphology. Briefly, the cells cultured on the bare PDMS substrate had a poor spreading and aggregated as their nuclei were marked with white squares in Figure 3-4. The cells cultured on the PLL-treated PDMS spread better, and no aggregation was observed as their nuclei were marked with white circles in Figure 3-4. 


\section{Bare PDMS}

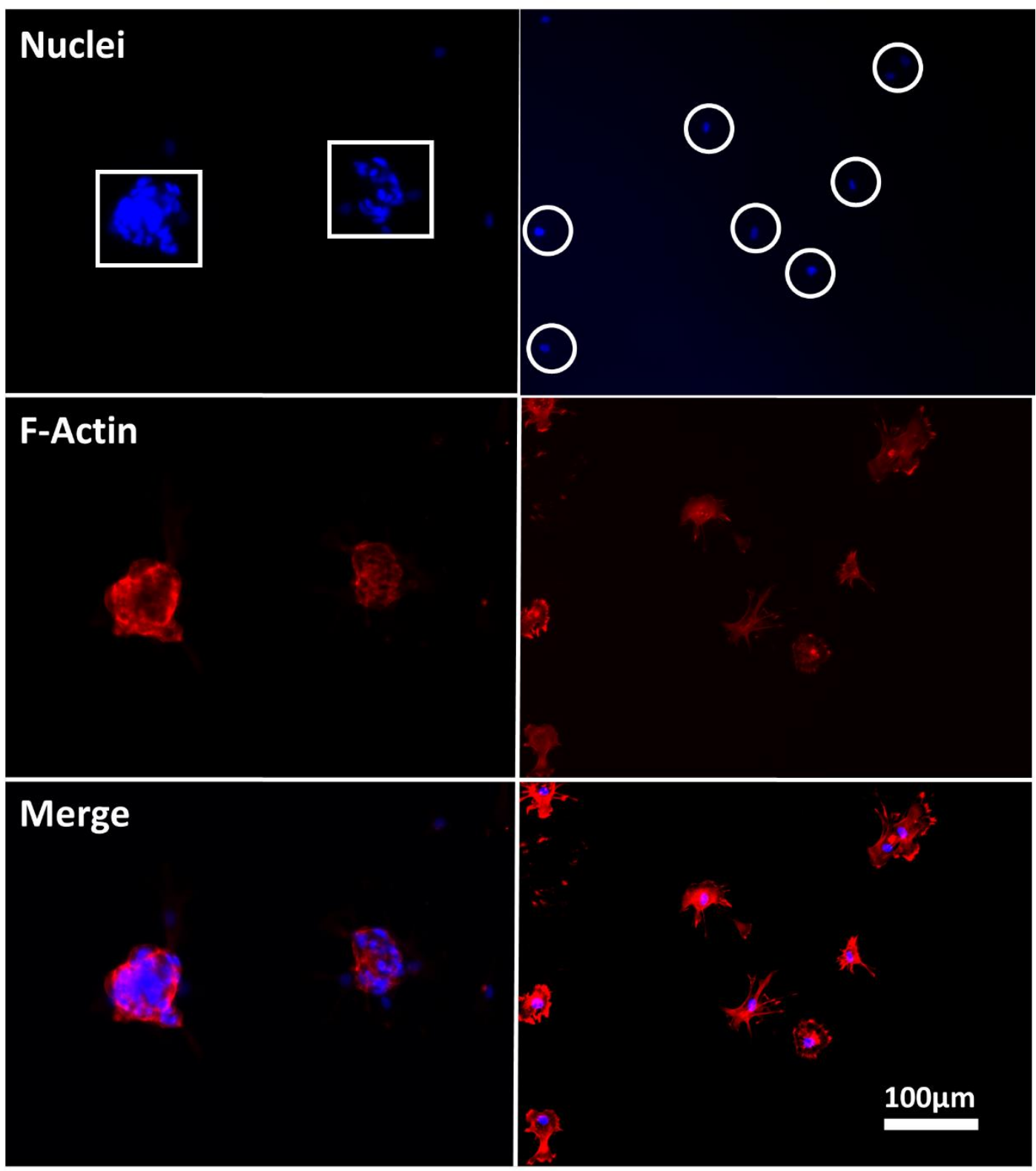

Figure 3-4 Fluorescent images of hMSC cultured on bare PDMS10 (Left) and PLL-treated

PDMS10 (Right) for one day. Red: Rhodamine Phalloidin stained F-actin; Blue: DAPI

$$
\text { stained nuclei. Scale bar: } 100 \mu \mathrm{m}
$$

Optical images of hMSCs on different PDMS were monitored throughout the experiment period (Figure 3-5). It could be observed that under current seeding density $(5,000$ cells per well), hMSCs were growing individually without clustering. Compared the initial 
growth stages (Figure 3-5(A) and (D)) with the later stages (Figure 3-5(C) and (F)), cells were separated away from each other on both PDMS5 and PDMS30 without clustering or forming a closely packed monolayer. Therefore, the measured cellular modulus should be affected by the PDMS stiffness even some cells were touching others.

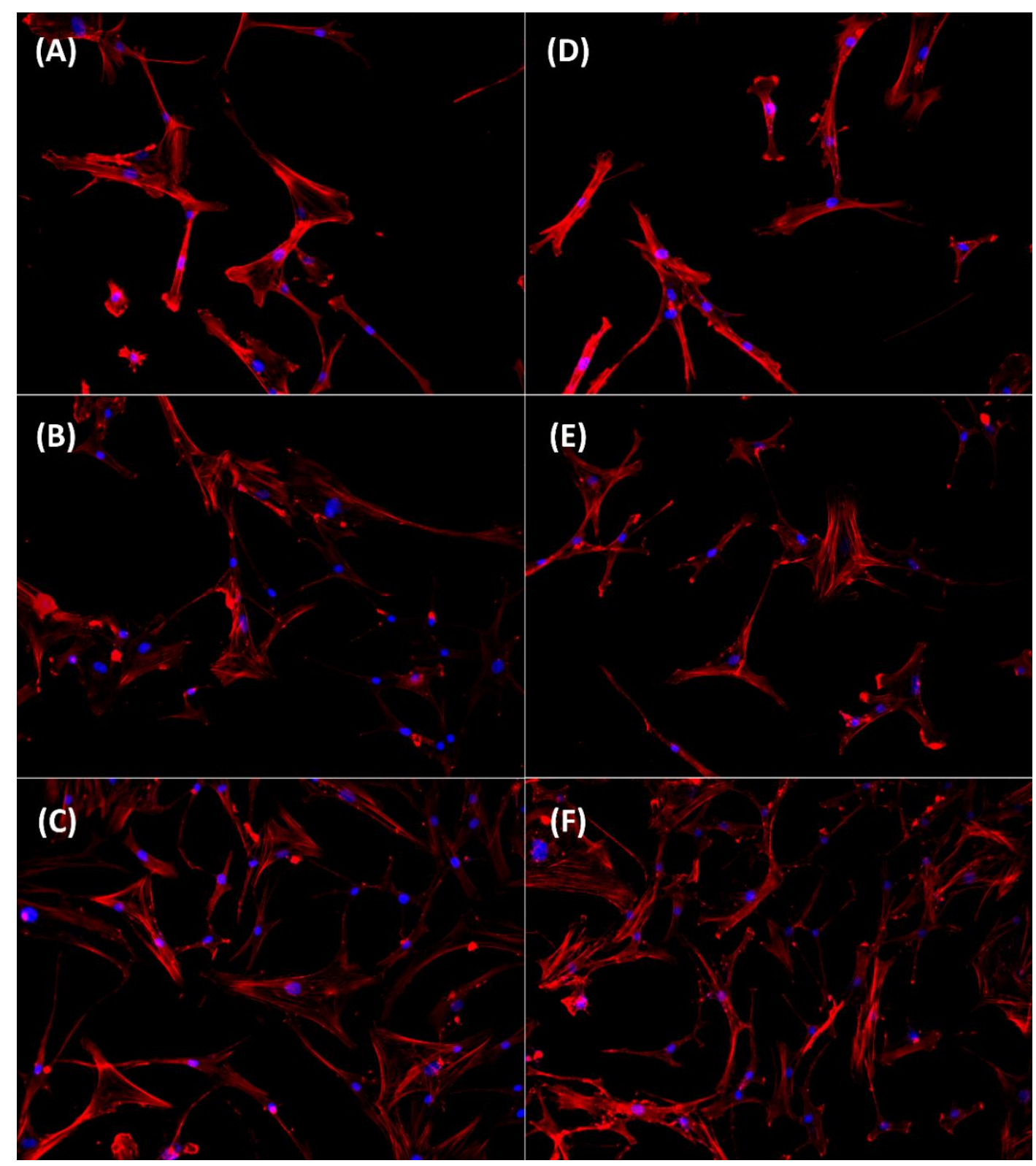

Figure 3-5 Fluorescent images of hMSC cultured on PDMS. Left: Day 5 (a), Day 11 (b) and Day 14 (c) on PDMS5; Right: Day 5 (d), Day 11 (e) and Day 14 (f) on PDMS30. Red: Rhodamine Phalloidin stained F-actin; Blue: DAPI stained nuclei 


\subsubsection{Elastic Property of hMSCs}

To evaluate the elastic property of hMSCs, elastic moduli of cells were calculated from the elastic model. In the situation when pipette radius is much smaller than cell radius ${ }^{228}$, the cell could be approximated as an incompressible elastic half-space ${ }^{243}$ and the simplest elastic model could be used to describe the cell aspiration behavior.

To examine the effects of PDMS stiffness and culture time on cell elastic moduli, the results derived from the elastic model were displayed (Figure 3-6). Results of the two-way ANOVA test gave the main effect value of PDMS stiffness, $F(3,40)=4.248, p=0.0107$ (see Section 3.3.7) and the main effect value of culture time, $F(4,40)=16.41, p<0.0001$, which indicated that both PDMS stiffness and culture time had significant influence on the elastic moduli of cells. In other words, it meant the cell moduli in the substrates with different stiffness were significantly different and the cell moduli tested on different culture days were also significantly different. However, the interaction between PDMS stiffness and culture time, with main effect value of $\mathrm{F}(12,40)=0.5742, \mathrm{p}=0.8495$, had no significant effects on elastic moduli. The influences of stiffness and culture time were independent. More precisely, the influence of substrate stiffness was consistent for different culture days and the influence of culture time was also consistent for substrates with different stiffness.

The elastic modulus of the hMSCs detached from the normal culture flask (Day 0) was the highest (Figure 3-6(A)), which was around $516 \mathrm{~Pa}$. On day 1, the elastic modulus of cells cultured on all PDMS substrates had similar values to each other around $100 \mathrm{~Pa}$, which decreased significantly from Day 0. This could be attributed to the large stiffness change 
from the culture flask $(\sim 1 \mathrm{GPa})^{246}$ to the PDMS substrates. On day 5, Young's moduli of cells were $155 \pm 76 \mathrm{~Pa}$ on PDMS5, $102 \pm 16 \mathrm{~Pa}$ on PDMS10, $41 \pm 5 \mathrm{~Pa}$ on PDMS20, and $35 \pm 6 \mathrm{~Pa}$ on PDMS30, which shows a clear trend that the cells cultured on stiffer substrates had higher moduli. On day 7, the similar trend could be observed except that the Young's modulus for PDMS20 was larger than that for PDMS10. On day 11, a significant difference of cell moduli between PDMS5 and PDMS30 was shown. Finally, on day 14, moduli of cells on different PDMS substrates became similar again, which were around $180 \mathrm{~Pa}$. In general, the moduli of the cells cultured on stiffer substrates were larger than the cells grown on softer substrates from day 5 to 11 , with exceptions found in only two groups, PDMS10 \& PDMS20 on day 7 and PDMS5 \& PDMS10 on day 11, among the 18 comparison pairs.
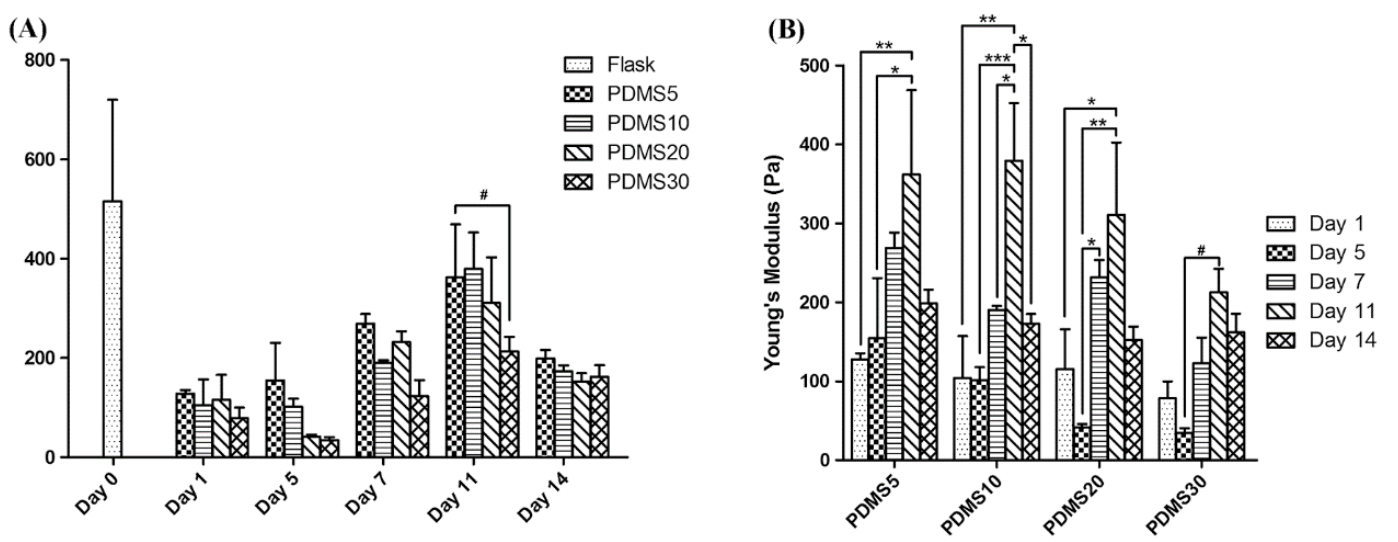

Figure 3-6 Young's modulus of cells calculated using elastic model ((A) modulus changing with time, (B) modulus changing with PDMS stiffness) $(*: p<0.05, * *: p<0.01$, $* * *: p<0.001$ and $\#: p<0.1)$

Besides the influence of substrate stiffness, the culture time played an even more significant role in the development of cellular moduli (Figure 3-6(B)). Overall, cellular 
moduli showed an increasing and then decreasing trend from day 5 to day 14 . The largest moduli were always achieved on day 11 , and the smallest moduli were observed on day 5 for cells on all PDMS substrates. As an example, for cells grew on PDMS5, which was the stiffest substrate, cell moduli increased from $155 \pm 76 \mathrm{~Pa}$ on day 5 to the maximum $362 \pm 107 \mathrm{~Pa}$ on day 11. After that, the value decreased to $199 \pm 17 \mathrm{~Pa}$ on day 14. Cell moduli on day 1 were larger than on day 5 except that on PDMS5, which probably indicated that how fast the cells could adapt to the substrate stiffness depended on how large the substrate stiffness was changed. In details, the experimental results indicate that it took more than 1 day for hMSC to adapt to the big change in substrate stiffness when transferred from the stiff flask to soft PDMS substrates (PDMS 10, 20 and 30). As a result, cells did not fully adapt to the soft substrate on Day 1 and would continue to reduce the moduli after that, which made cellular moduli larger on Day 1 than Day 5. While for PDMS5, which was more than 2 times stiffer than other PDMS substrates, it took less time for hMSC to adapt to the substrate stiffness change so that cellular moduli could show an increase from Day 1 to Day 5.

\subsubsection{Viscoelastic Properties of hMSCs}

To further investigate the cellular mechanical properties change, a more precise model was used. hMSCs in the experiments showed typical viscoelastic behavior during aspiration. Right after the step pressure being applied, there was an aspiration length jump. Following the initial jump, the aspiration length increased slowly under constant pressure before reached the equilibrium length (Figure 3-7). Through fitting the time-dependent aspiration 
length using the viscoelastic model, three relevant mechanical parameters, $E_{0}, E_{\text {eqm }}$, and $\mu$ could be obtained (Figure 3-8 and Figure 3-9). All the aspiration length data were fitted using the viscoelastic model as explained in the methods section (Equation 3-2). Most fittings showed high accuracy with the mean correlation coefficients higher than 0.9 . The worst fitting had a mean correlation coefficient around 0.71 , which was probably due to the break of the cell membrane during aspiration process. Overall, results showed that the instantaneous Young's modulus of cells (in the range of hundreds or even thousands of Pascal) were larger than their equilibrium Young's modulus (from tens to hundreds of Pascal). And most of the apparent viscosity values were in the range of thousands of Pascal*second. 

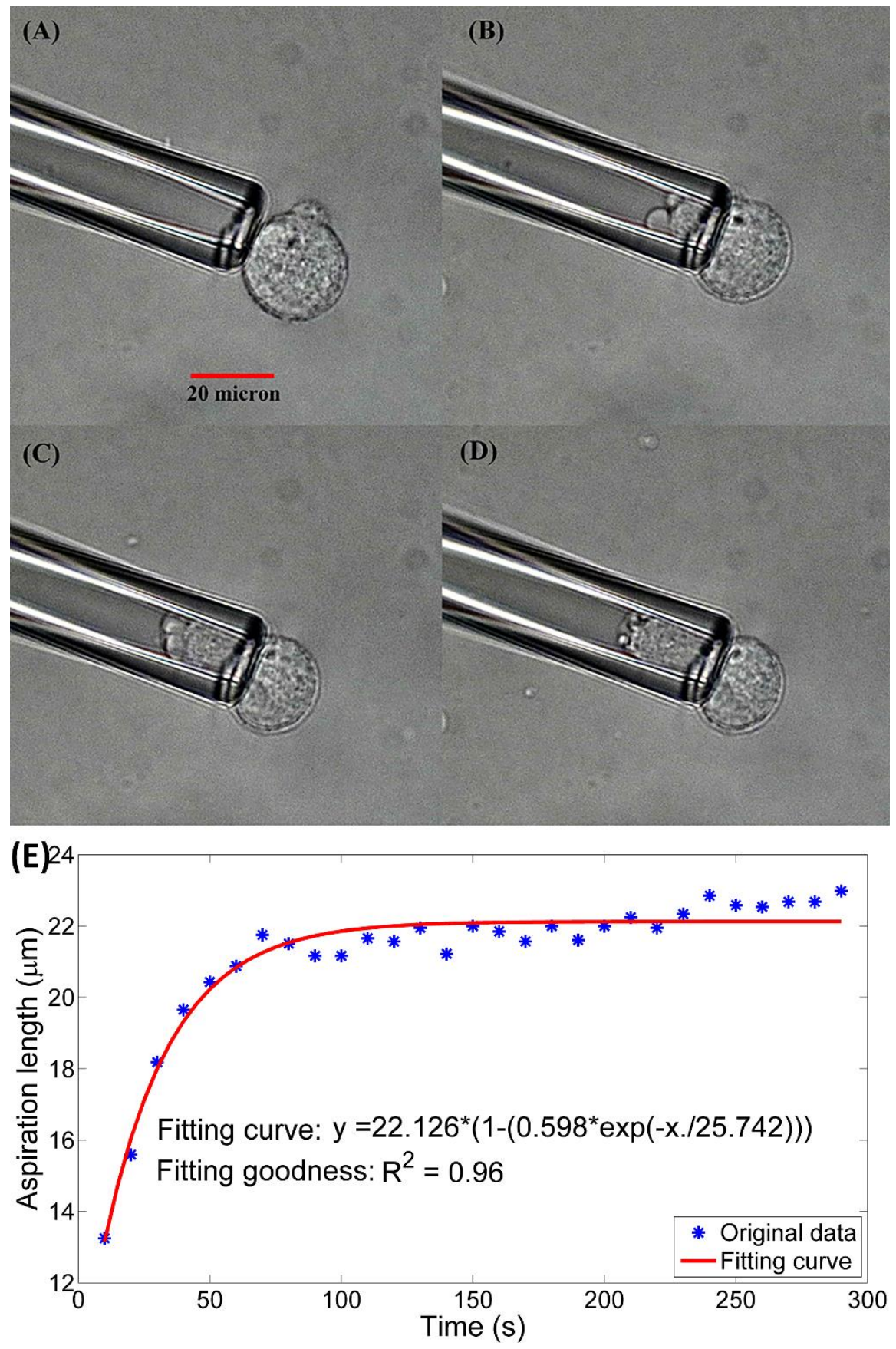

Figure 3-7 Aspiration of cells in micropipette at different time points. (A) the pressure was adjusted to reach equilibrium, (B), (C), and (D) were $0 \mathrm{~s}, 100 \mathrm{~s}$, and $300 \mathrm{~s}$ after applying the step aspiration pressure, and (E) was the corresponding fitting curve of the cell aspiration length as a function of time

The main effects of PDMS stiffness and culture time on the three viscoelastic 
parameters, $E_{0}, E_{\text {eqm }}$, and $\mu$ were evaluated as well using two-way ANOVA method as mentioned in the section 3.3.7. Significant influence of both PDMS stiffness $(\mathrm{F}(3,40)=6.16, p=0.0015)$ and culture time $(\mathrm{F}(4,40)=11.30, p<0.0001)$ on instantaneous moduli $E_{0}$ had been demonstrated. Similarly, PDMS stiffness $(\mathrm{F}(3,40)=3.767, p=0.0180)$ and culture time $(\mathrm{F}(4,40)=18.38, p<0.0001)$ showed significant effects on the $E_{\text {eqm }}$. However, the interaction between PDMS stiffness and culture time showed no significant effect on $E_{0}$ and $E_{\text {eqm }}$. Furthermore, effects of PDMS stiffness, culture time, and their interaction on $\mu$ were all insignificant.

A strong dependence of the cell mechanical properties on PDMS substrate stiffness could be noticed, which is the same trend as the elastic model but more obvious (Figure 3-8). In general, the viscoelastic parameters of cells cultured on a stiffer PDMS were usually larger than those cultured on a softer PDMS from day 5 to 11 and the differences were larger than the elastic modeling results. Only 5 exceptions where cells had larger moduli or viscosity on softer PDMS were observed among the 54 one-to-one comparisons of all three viscoelastic parameters from day 5 to 11 between cells cultured on different PDMS substrates. It could be seen the 5 exceptions were all non-significant in statistic. The most exceptions (4/5) were found between cells on adjacent PDMS substrates and no significant difference could be found between cells on adjacent PDMS substrates. It probably indicated the difference of stiffness between adjacent substrates (i.e. PDMS5-PDMS10, PDMS10-PDMS20, and PDMS20-PDMS30) was not enough to be sensed by cells. 
(A)
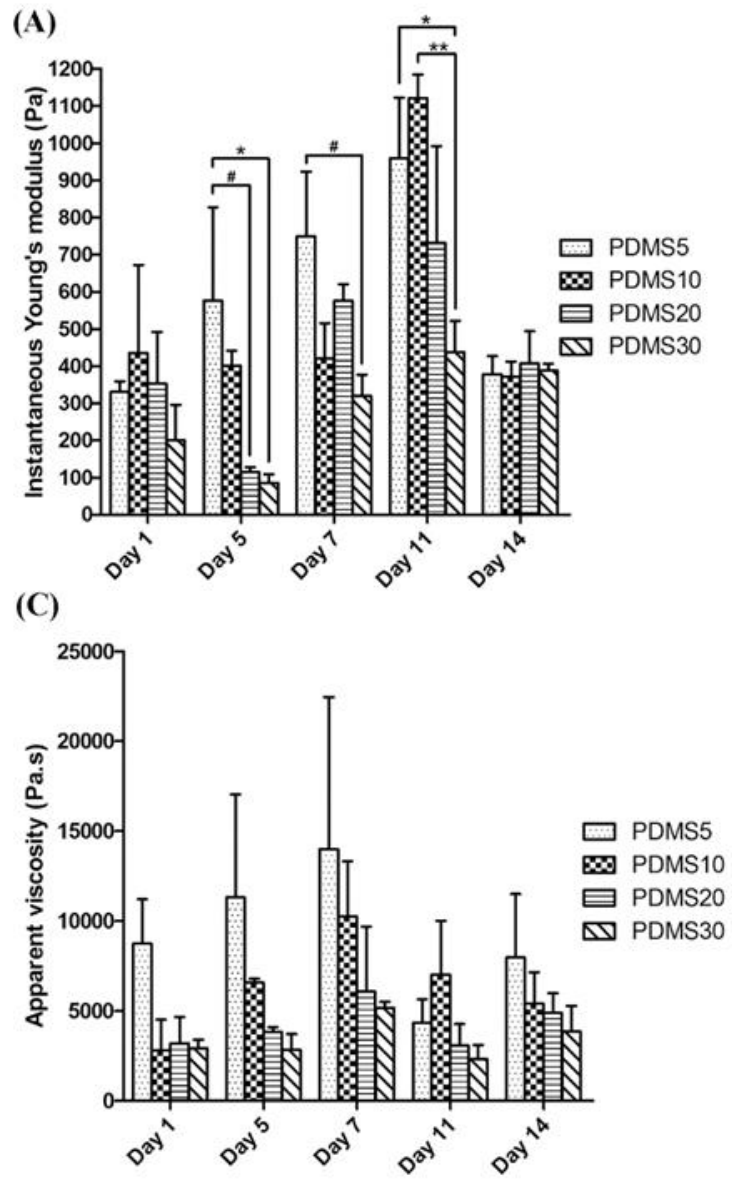

(B)

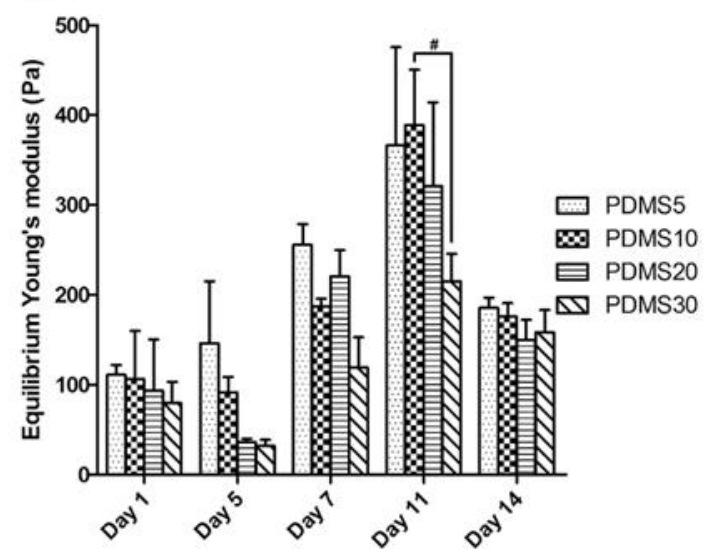

Figure 3-8 Cell viscoelastic parameters influenced by PDMS substrate stiffness ((A), (B), and (C) were figures for $E_{0}, E_{\text {eqm }}$, and $\mu$, respectively as functions of culture days) (*:

$$
p<0.05, * *: p<0.01, * * *: p<0.001 \text { and } \#: p<0.1)
$$

The influence of cell culture time on the three mechanical parameters was also studied

(Figure 3-9). In the two-week experimental duration, the change of the instantaneous and equilibrium moduli with time was similar as the elastic model results. The instantaneous Young's moduli of cells cultured on PDMS5 increased first from day 1 to day 11 and then decreased on day 14, while instantaneous Young's moduli of cells cultured on other PDMS substrates decreased first from day 1 to day 5 , then increased from day 5 to day 11 , and finally decreased again at day 14. Similarly, the difference of PDMS5 on day 1 may 
be attributed to the shorter time required for cells to adapt to the new substrate with smaller stiffness change. The same phenomenon was shown for the equilibrium Young's modulus. For both $E_{0}$ and $E_{\text {eqm }}$, similar values were reached on Day 14 regardless of substrate stiffness. Different from the trends of $E_{0}$ and $E_{\text {eqm }}$, there is no any significant difference for cell apparent viscosity values at different time points.
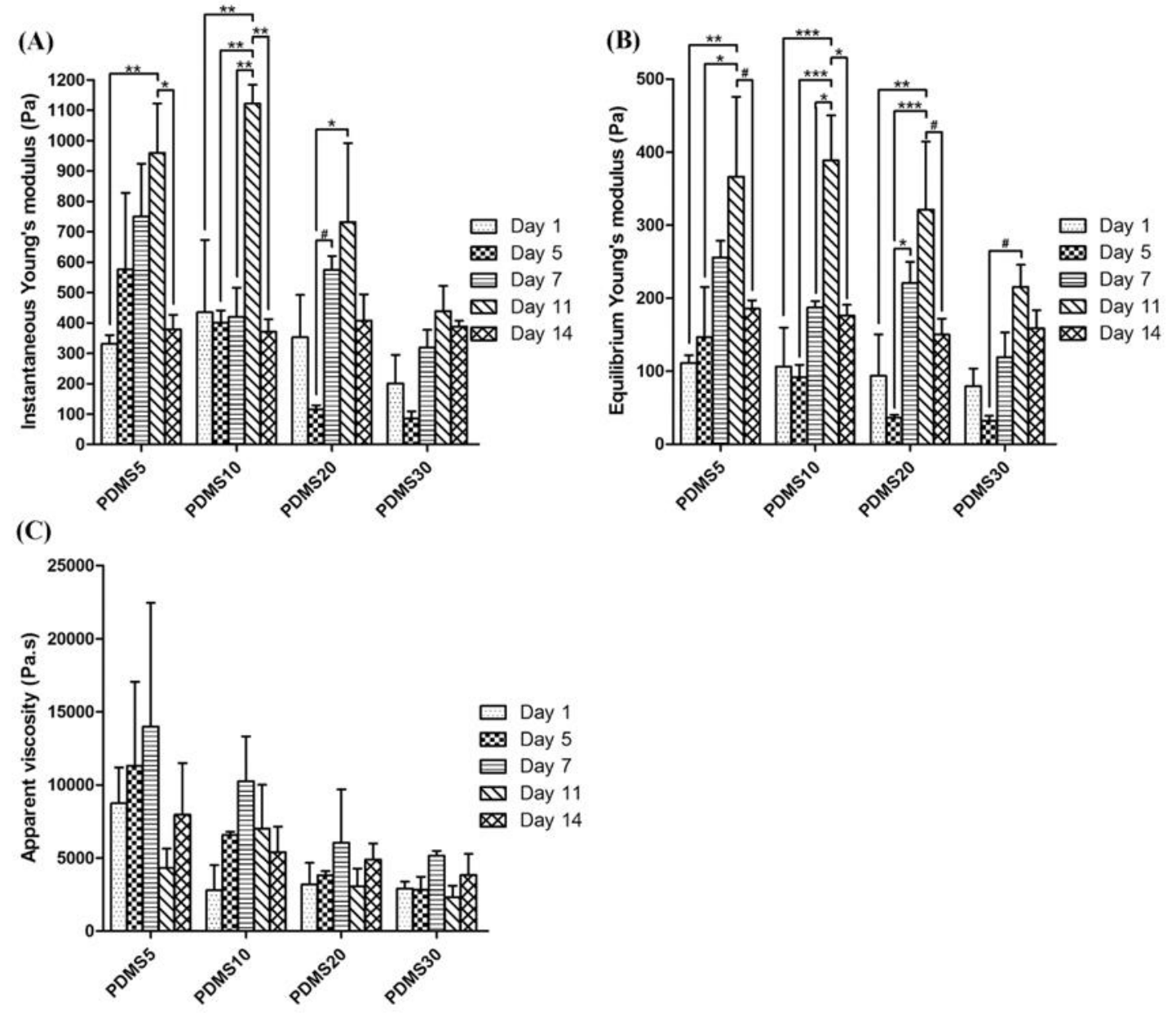

Figure 3-9 Cell viscoelastic parameters as influenced culture time ((A), (B), and (C) were figures for, $E_{0}, E_{\text {eqm }}$, and $\mu$, as functions of PDMS stiffness) (*: $p<0.05, * *: p<0.01, * * *$ :

$$
p<0.001 \text { and } \#: p<0.1)
$$




\subsubsection{Osteogenic Specification of hMSC}

According to the previous study ${ }^{70}$, substrates that mimic specific tissues could direct MSC lineage specification. Since the PDMS substrates were stiff and close to collagenous bones, we hypothesize that they could be osteogenic. One osteoblast marker, anti-RUNX2 antibody, was chosen to characterize the differentiation of MSCs to osteoblasts. Alizarin Red was chosen to evaluate the biomineralization by staining the calcium deposits.

The RUNX2 expression and Alizarin Red staining on PDMS5 and positive control groups were shown in Figure 3-10 (a). On day 1, all the cells on PDMS5 grew separately, and no RUNX2 expression could be observed in the image. After 11-day culture, on which day the cells had highest moduli, osteoblast-like multiple-nuclei cells and RUNX2 expression could be observed. On day 14, the RUNX2 expression was also observed. However, most cells grew separately, and no RUNX2 expression was detected in them. Compared to cells cultured on PDMS5 with normal culture medium, cells from positive control groups, which were cultured on PDMS5 with the osteogenic medium, formed more osteoblast-like cells. These cells aggregated together and formed multiple-nuclei cells. The results of Alizarin Red staining were shown in Figure 3-10 (b)(c)(d)(e). No calcium deposits could be found on PDMS5 on day 1 (Figure 3-10 (b)). On day 11 (Figure 3-10 (c)) and day 14 (Figure 3-10 (d)), some cell aggregations were found and stained with Alizarin Red. The same phenomenon could also be found on cells cultured on all the PDMS samples (not shown in the figure), so PDMS5 was chosen as an example. On day 14, most of the cells cultured on PDMS5 with osteogenic medium (Positive Control) had differentiated into the osteoblast-like cells that formed bone nodule-like structures (marked by the arrows in 
Figure 3-10 (e)). It indicated some cells on PDMS had differentiated into osteoblast-like cells with two-week culture. 


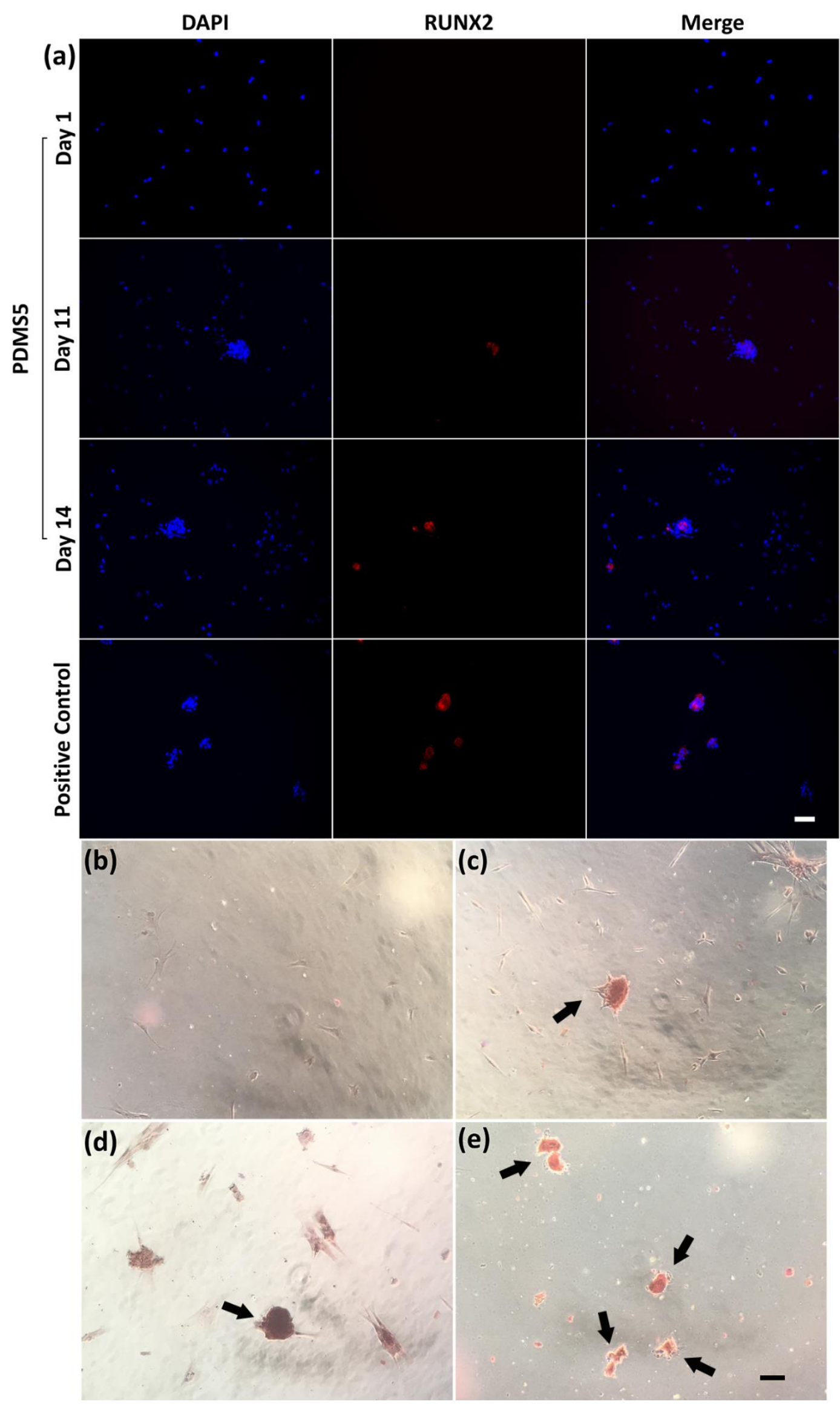

Figure 3-10 Osteogenic specification with culture time. (a) RUNX2 expression on PDMS5 
(Day 1, Day 11 and Day 14) and Positive Control Group (cells cultured on PDMS5 with osteogenic medium on Day 14). Red: RUNX2; Blue: DAPI stained nuclei. Assessment of biomineralization on PDMS5 on day 1 (b), day 11 (c), day 14 (d) and on Positive Control Group on day 14 (e) revealed by Alizarin red staining. The arrow indicates some cell aggregations. Scale bar: $100 \mu \mathrm{m}$.

To quantify the differentiation ratio on different PDMS substrates and different culture day, the percentage of RUNX2 expression in nuclei, which was defined as the number of nuclei with RUNX2 expression (spots stained by both blue and red) divided by the total number of nuclei in the image (spots stained by blue) (processed by ImageJ), were shown in Figure 3-11. Three images were chosen for each data point.

On day $11,9.89 \% \pm 13.45 \%, 3.39 \% \pm 3.37 \%, 4.51 \% \pm 2.72 \%$ and $1.63 \% \pm 2.82 \%$ cells expressed RUNX2 on PDMS5, PDMS10, PDMS20 and PDMS30 respectively. On day 14, $13.42 \% \pm 6.47 \%, 5.30 \% \pm 5.09 \%, 7.40 \% \pm 6.52 \%$ and $6.96 \% \pm 7.15 \%$ cells expressed RUNX2 on PDMS5, PDMS10, PDMS20 and PDMS30 respectively. Although a slight increase of RUNX2 expression from day 11 to day 14 was found on all PDMS substrates, there is no statistical difference. Furthermore, the differences of RUNX2 expression between different PDMS substrates on both day 11 and day 14 were not statistically significant. The only significant difference was found between the positive control groups and corresponding experimental groups. 


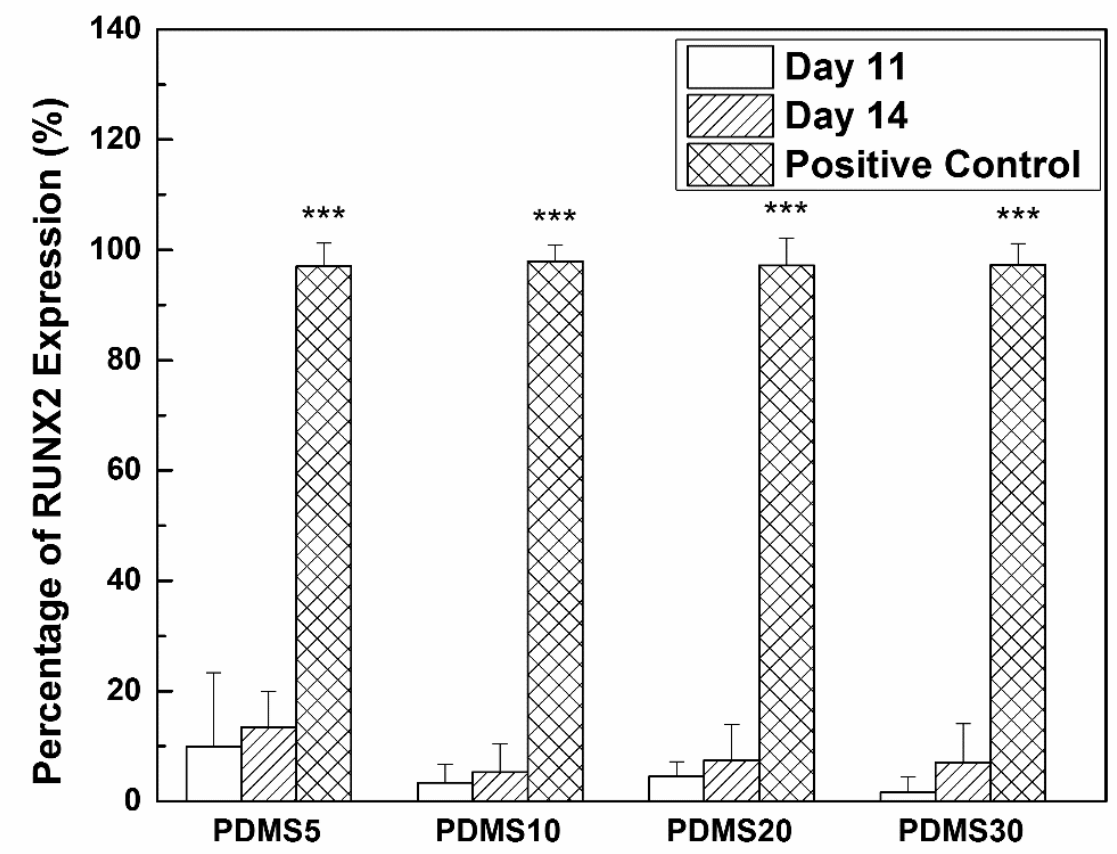

Figure 3-11 Percentage of RUNX2 expression on different PDMS substrates (Day 11, Day 14) and corresponding positive control groups (Day 14). All values were mean \pm standard deviation. $* * *$ indicates $\mathrm{p}<0.001$.

\subsection{Discussion and Conclusions}

Because the trends of cellular moduli change were almost same in both elastic and viscoelastic model. To simplify the comparison, the moduli in elastic model were chosen for the discussion.

\subsubsection{Cellular Mechanical Properties vs. Substrate Stiffness}

Based on the results, a strong dependence of the cell mechanical moduli on substrate stiffness could be observed, regardless of which model was used. In general, cell 
mechanical moduli tended to be larger on stiffer substrates, similar to the previous works $^{52,247}$. This tendency of cells tuning their stiffness to match that of the substrates they attached was consistent to the previous studies ${ }^{52}$. This phenomenon has been reported to be attributed to increased cytoskeletal assembly, activation of crosslinkers, and generation of internal tension which is related to the strain-stiffening non-linear elasticity of the cytoskeleton in others' work ${ }^{247}$. Since a similar work measured a higher concentration of PLL $(0.5 \mathrm{mg} / \mathrm{ml})$ coating on a plasma-treated silicon surface (glass) resulted in $1 \mathrm{~nm}$ thickness, the PLL coating thickness in our study $(0.1 \mathrm{mg} / \mathrm{ml}$ on another silicon surface, PDMS) would be in the same range. Furthermore, previous work (in the supplementary materials) demonstrated the MSCs can sense the stiffness of substrate even with $500 \mathrm{~nm}$ gel coating on that substrate. So, in a very thin coating of PLL, the cells could sense the substrates easily.

To investigate the relation between cellular moduli and substrate stiffness, we observed the morphology of cells on different substrates. On day 11 , when the highest cellular moduli were measured, the morphology of cells cultured on PDMS5 and PDMS30 was shown in Figure 3-12 (a) and (b). Although most of the cells had a polygonal shape, the poor spreading area could be observed in some cells. It could be noticed that the number of cells had poor spreading was higher on the PDMS30, the softer substrate. It indicated a positive correlation between spreading and substrate stiffness. In other words, cells spread better in a stiffer surface. It clearly showed actin stress fibers in fully spread cells (Figure 3-12 (c)) whereas no visible actin stress fibers could be seen in cells with poor spreading (Figure 3-12 (d)). It provided evidence the spreading area is related to the actin stress fiber 
formation, which is a key factor in determining the cellular stiffness ${ }^{52}$.

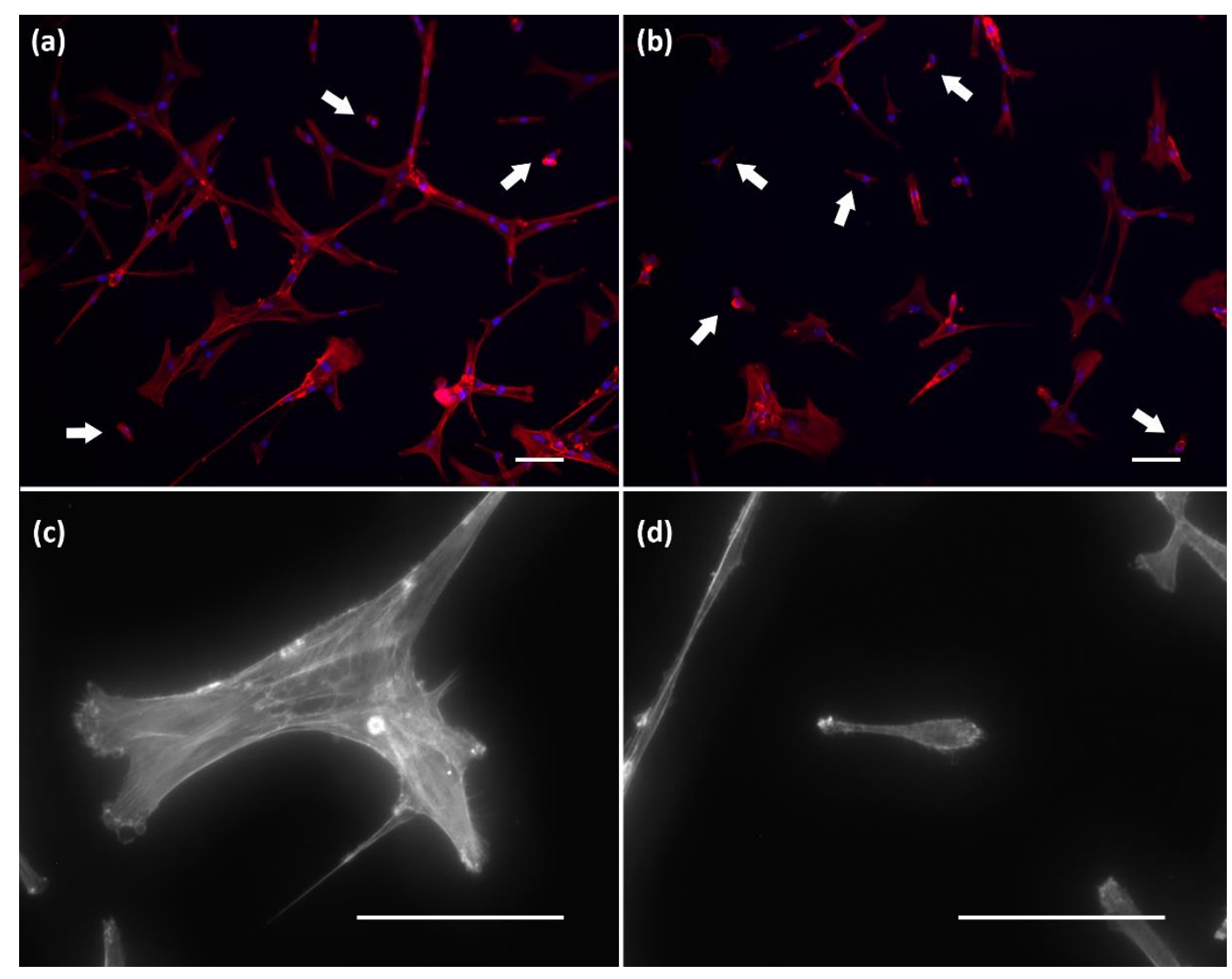

Figure 3-12 MSCs cultured on PDMS5 ((a), (c), (d)) and PDMS30 (b) on day 11. The cells had poor spreading were marked by white arrows. Red: Rhodamine Phalloidin stained F-actin; Blue: DAPI stained nuclei. (c) and (d) showed the F-actin only. Scale bar is 100

$\mu \mathrm{m}$.

\subsubsection{Cellular Mechanical Properties vs. Culture Time}

The other important phenomenon was that cellular moduli as functions of time displayed a non-monotonic trend regardless of PDMS substrate stiffness, which could be classified into three different zones. First could be called adapting zone, where cellular moduli decrease at the very beginning to adapt to the new substrates after taking out from a flask and the adapting time required is proportional to the difference of the two substrates in 
stiffness. Second is the growth zone, where cellular moduli increased to the maximum values. Previous studies have shown an increasing stiffness of individual human epithelial cells with aging in vitro ${ }^{248}$, which was attributed to a higher density of cytoskeletal fibers in the older cells than those in the younger cells that led to the increase of cellular modulus. The similar results could also be observed in our experiment as shown in Figure 3-13. The cytoskeletal fiber was coarsening with increasing culture time (from Day 1 to Day 14) and this trend was independent of substrate stiffness. The last zone is the convergent zone, where cellular moduli decrease again to similar values regardless of substrate stiffness. However, we didn't see any visible difference in cytoskeletal actin from Day 11 to Day 14. The mechanism of this moduli drop from Day 11 to Day 14 needs further investigated. Importantly, a previous study indicated this cytoskeletal coarsening and stiffening in MSCs were not associated with any detectable changes in selected surface marker expression and attendant with reduction in population differentiation capacity ${ }^{249}$. 


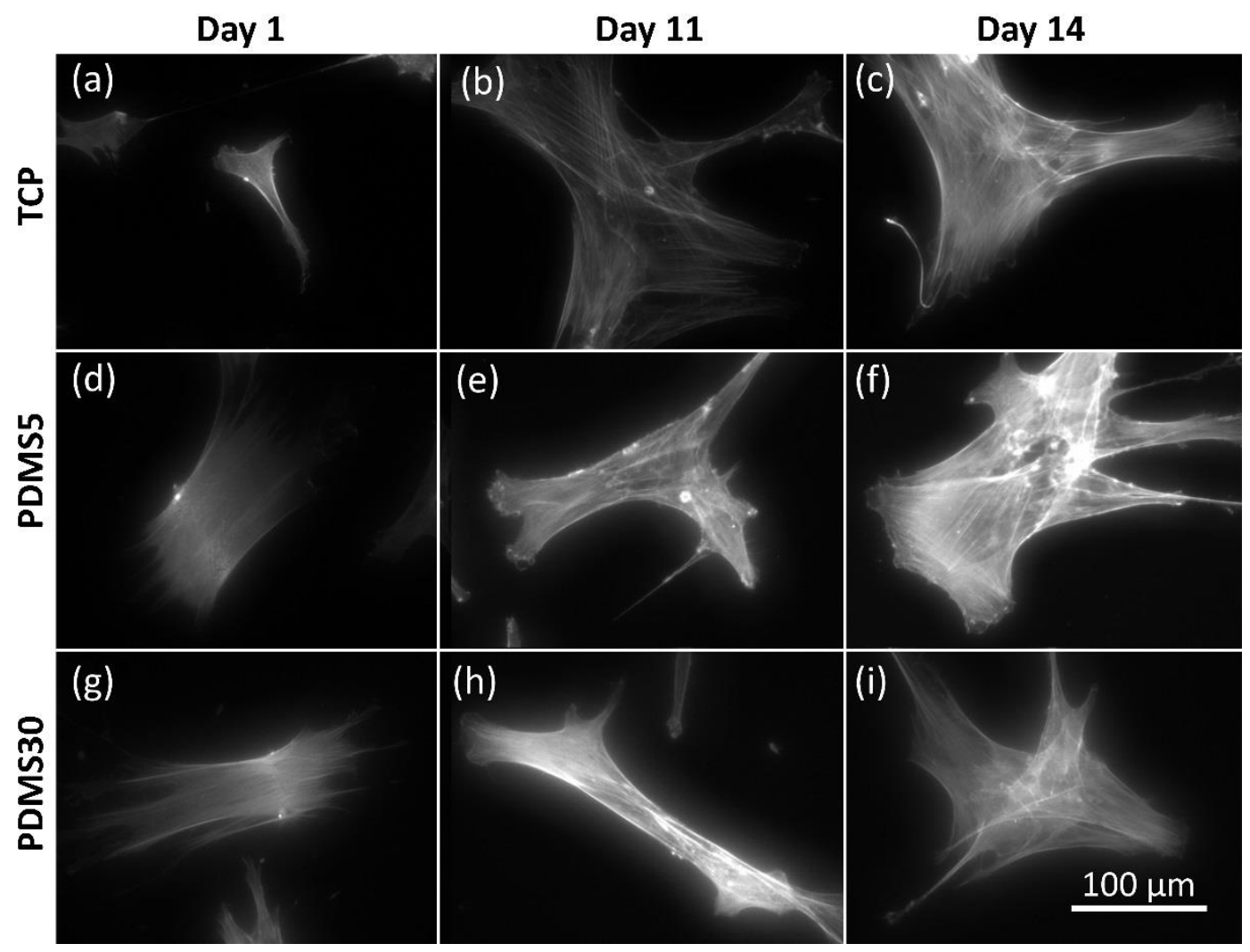

Figure 3-13 F-actin staining of MSCs show cytoskeletal coarsening with increasing culture (Day1, Day11 and Day14) time on different substrates (TCP, PDM5 and PDMS30).

Previous studies have demonstrated that cells could respond to the physical properties, including stiffness and topography, of the substrates ${ }^{52,70,247,250}$. The change of cellular mechanical properties was probably attributed to the lineage specification. It was suggested that the differentiation behavior of MSCs could be related to the stiffness of substrates $^{70}$. On the softest substrates, the highest expression of neurogenic transcripts in MSCs was observed by upregulating neuron-specific cytoskeletal markers such as nestin $\beta_{3}$ tubulin, neurofilament light chain (NFL), and protein NCAM. On moderately stiff substrates, MSCs express the most myogenic message with upregulation of proteins such as Pax activators and myogenic factors (e.g. MyoD). On the stiffest substrates, MSCs 
express the greatest osteogenic message with upregulation osteocalcin and transcriptional factor $\mathrm{CBF} \alpha 1$. To investigate whether the cellular moduli change in our study was related to lineage specification, immunocytochemistry staining of transcriptional factor CBF 1 (RUNX2) was used. The lowest substrate stiffness in this study was $60.75 \pm 8.62 \mathrm{kPa}$, which is already in the range that could direct MSCs to differentiate into osteogenic lineage ${ }^{70}$. After the immunostaining of RUNX2 (CBF $\left.\alpha 1\right)$, we did find some cells differentiated into osteoblast-like cells. However, only $5.30-13.42 \%$ of cells showed RUNX2 expression while around $98 \%$ of cells on corresponding positive control groups showed RUNX2 expression after two-week culture. A previous study found MSCs generally decreased their Young's moduli during chemically-induced osteoblast differentiation ${ }^{251}$. It has been attributed to the cellar structure changes during the differentiation ${ }^{252,253}$. The cytoskeleton structure change could be observed in this experiment as shown in Figure 3-14. In hMSCs, actin stress fibers were long, parallel, and oriented (Figure 3-14 (a)); cytoskeletal rearrangement during osteoblast differentiation resulted in disordered actin filaments and no visible actin stress fibers could be seen (Figure 3-14 (b)). Here, we proposed a possible explanation that the cellular moduli drop from day 11 to day 14 was caused by differentiation induced softening. In the beginning, cellular moduli increased with the cytoskeletal coarsening. After certain days, some cells were differentiated. At the same time, some other cells were differentiating but had not expressed RUNX2 due to the unsynchronized differentiation process. Because the moduli of MSCs decrease during osteogenic differentiation ${ }^{251,252}$. These differentiated and differentiating cells would have lower moduli compared to normal MSCs. The existence 
of differentiated and differentiating cells could provide an explanation for the moduli drop from day 11 to day 14. This explanation of differentiation-induced moduli drop will be enhanced if the ratio of the differentiated cells and the differentiating cells in the whole cell culture is known. However, our study found this unreported time-dependent change of cell moduli and offered a possible explanation.
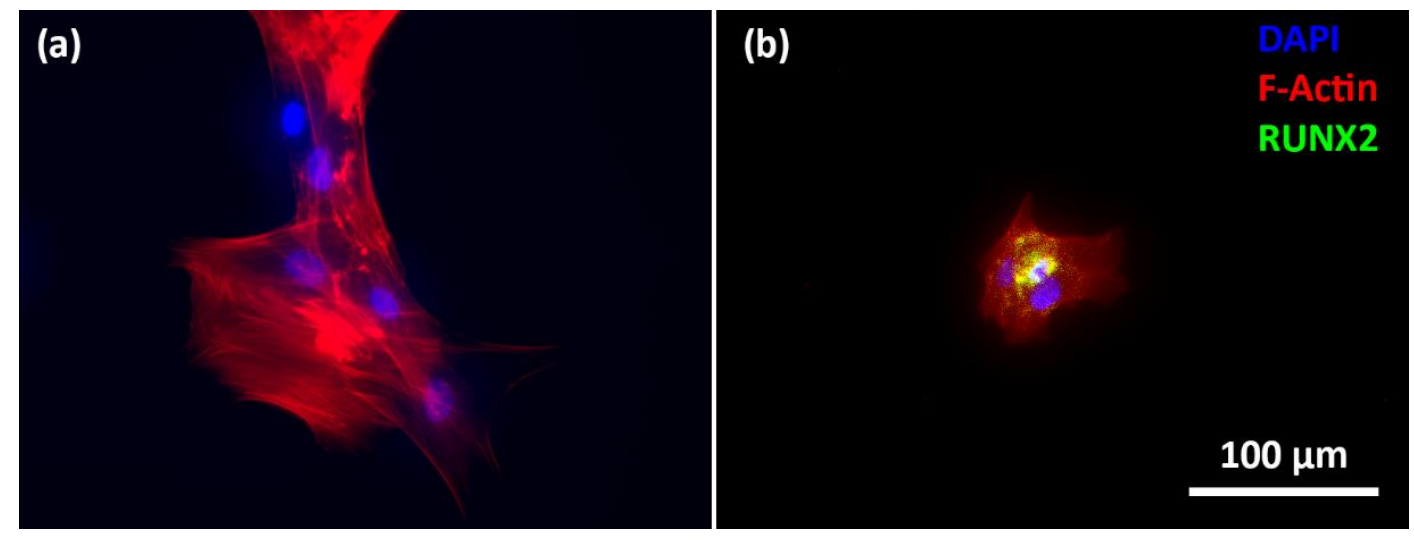

Figure 3-14 F-actin structure of MSCs without RUNX2 expression (a) and differentiated

Osteoblast-like cells (b) cultured on PDMS10 for 14 days. Red: Rhodamine Phalloidin stained F-actin; Blue: DAPI stained nuclei; Green: RUNX2. Scale bar: $100 \mu \mathrm{m}$.

Another factor probably involved in the change of cellular mechanical properties is cell-cell interaction. In our project, we used the same seeding density to ensure the cells tested on different days had the same initial growth condition. As shown in Figure 3-5, some cells were touching with others. A slight increase in cell contact could be observed from Figure 3-5 during two-week culture when the cellular moduli increased as well. People had found the cell-cell contact could improve the spreading and increase the formation of stress fibers ${ }^{254}$. So, the increasing cell-cell contact of MSCs in our study probably contributed to the moduli increase with time. On the other hand, a recent study of MSCs revealed the nuclear localization of transcription factors associated with 
osteogenesis, like RUNX2, depended on substrate stiffness and was independent of the degree of cell-cell contact ${ }^{255}$. So, cell-cell contact would not decrease the moduli with culture time by improving osteo-differentiation. In summary, the cell-cell contact probably contributed to the moduli increase with time but did not contribute to the moduli decrease in our study, although further evidence would enhance our explanation.

\subsubsection{Conclusion}

Change of cell mechanical properties, such as elasticity and viscoelasticity, have attracted lots of interest due to its relationship with various cellular behaviors, like proliferation, adhesion, and differentiation ${ }^{215}$. However, the influence of culture time should also be very important even though few studies could be found in this aspect, and this work highlighted the effect of both substrates stiffness and culture time on mechanical properties of the cell. The change of cellular mechanical properties on different PDMS substrates is probably attributed to the difference of cell spreading on different substrates. A stiffer substrate improved the cell spreading and resulted in a stiffer cell. The simplest explanation is that cells have a binary sensor on the membrane junction sites that signals for a relaxed round morphology when the surface is softer than the cell's intrinsic elastic modulus, and signals for another phenotype with increased contractility and stress fiber formation when the external material is stiffer.

The change of cellular mechanical properties with culture time is more complex. One of the reasons is the cytoskeleton coarsening with time. Another reason is probably the osteogenic specification directed by the stiff substrate with culture time. The 
differentiation was low efficient compared to normal chemically-inducted differentiation, so the skeleton structure change induced by osteogenic differentiation was slow. The cellular mechanical properties changed with time could be explained as follows: at the beginning, the cellular moduli increased with time due to the cytoskeletal coarsening. After certain days, some cells became differentiated cells (based on the observation of RUNX2 expression), and some other cells were differentiating but had not expressed RUNX2 due to the unsynchronized differentiation process. Since the cells' moduli decreased during the osteo-differentiation, these cells, including differentiated and differentiating cells, had lower moduli compared to normal $\mathrm{MSCs}^{251,252}$. So, the moduli change would not only happen in the differentiated cells, which could explain the moduli drop from day 11 to day 14 . Although a further study would enhance this explanation, we offered a possible explanation for this unreported cell moduli change with culture time.

In summary, this study revealed a new phenomenon that cellular moduli change with culture time after seeding on the substrates besides the cellular moduli change with substrate stiffness. This change of cell moduli observed through long-term culture indicated that cell stiffness should be modulated not only by substrate stiffness but also by culture time. According to the $p$ values of main effects of PDMS and culture time, the impact of the culture time on the development of mechanical properties of hMSCs was greater than that of PDMS stiffness, i.e., culture time could have even more significant effects on cellular moduli than substrate stiffness. Furthermore, it provides new evidence that cells cultured in the stiff substrates $(60 \mathrm{kPa}-1.2 \mathrm{MPa})$ could inducted into osteoblast-like cells without chemical induction, although the ratio is low. It enriched the 
understanding of physically-inducted differentiation and provided a promising indicator for cell aging and differentiation. However, the mechanism of the cell moduli change needs further investigation, especially why the cells in different substrates had similar moduli after two-week culture. 


\section{Chapter 4. Influence of Dynamic Loading on hMSCs Cultured in 3D Nanofibrous Scaffolds}

\subsection{Significance and Novelty}

Dynamic cell culture in 3D scaffolds is very promising and important to mimic the cell growth state in their native extracellular matrix (ECM). Hydrogels and 2D mats have been used to transmit mechanical loading to cells. However, the lack of 3D structures limited the application of 2D samples in compression, and the cell-laden hydrogel would decrease the viability of cells due to the high temperature needed in the cell seeding (agarose) ${ }^{256}$ or photopolymerization (usually triggered by UV or laser) ${ }^{64}$. So, a 3D scaffold with desired mechanical property and easy processing for cell culture are needed.

In this study, the use of 3D polypyrrole-coated polyacrylonitrile nanofibers, which were easily fabricated with electrospinning and had a proper mechanical property, to applying the dynamic compression in cell culture is new. The elasticity and the $3 \mathrm{D}$ structure of the scaffold allowed the 3D cell culture with dynamic compression. The direct cell seeding without heating and polymerization (different from gels) avoids harmful stimulus. Different from the electrospun yarns in other study ${ }^{257}$, the 3D scaffolds in this study could be fabricated in an easier method and have larger inner space to help cell infiltration. The cell proliferation, morphology and collagen expression of hMSCs cultured in the dynamic 3D-CNFs were characterized over a long-term culture period ( 2 weeks). Results indicated the MSCs entered the interior of the scaffold and stable cell-laden construct was formed. 
The rate of the proliferation was maintained, and collagen production was promoted compared to that in static 3D scaffolds and flat tissue culture plates.

\subsection{Introduction}

Tissue engineering has been recognized as a promising solution to repair or replace portions of or whole tissues. To achieve the goal of producing a clinically useful tissue, various structures of engineered scaffolds have been developed. The main strategy is to build a tissue-engineered construct in vitro by seeding cells in a scaffold that could be used as an implant in clinical applications later; however, there are significant challenges for developing tissues with appropriate structure and composition. Recently, a lot of efforts have been made on the functional fibrous scaffolds with high porosity and high spatial interconnectivity ${ }^{37,258-260}$ to produce appropriate structures.

In recent years, 3D nanofibers (3D-NFs) have been explored for various beneficial applications in the field of tissue engineering ${ }^{261-264}$. These 3D micro/nanofibrous materials are characterized by deep, interconnected pores, and are fabricated using various synthesized and natural polymers. The effective performance of the 3D micro/nanofibrous structure is often defined by choice of polymers, their innate mechanical and chemical properties and the interconnected microscopic architectures. In supporting tissue regeneration, it is believed that continuous macro-porous structure, matching the natural mechanical strength and mimicking the dimension scale of ECM constitute an overall microenvironment for long-term cell survival and generation of functional tissue ${ }^{265-272}$. In this context, preparing 3D-NFs with a continuous, open porous structure and precise 
control of the biochemical and mechanical properties could be a novel strategy to achieve promising performance for a series of applications. To realize these properties, several approaches including electrospinning, carbonization, and self-assembly methods have been used in the fabrication of such nanofibers ${ }^{272-276}$. Recently, researchers in this area have focused on the developing novel preparation techniques for 3D electrospun nanofibers generation, which demonstrated highly porous interconnectivity and open porosity, and exhibited various applications in tissue engineering ${ }^{273,277}$. Despite their potential in tissue engineering, the $3 \mathrm{D}$ nanofibers do not fairly recapitulate the microenvironment as the ECM with hierarchically organized and dynamic nanofibrous constructs regulate essential cellular functions. Moreover, most of these nanofibers are not completely 3D structured fibrous scaffolds but rather 2D nanofiber mesh deposits, which demonstrate poor mechanical strength or unsuitable pore size for cell infiltration and storage. Therefore, it is pivotal to construct homogenous and mechanically viable 3D-NFs capable of forming an interconnected structure with desirable pore size, which could enhance the functionality of the intrinsic nanofibers to be widely applied.

Polyacrylonitrile (PAN) was known to be a safe material for medical use such as drug delivery, wound dressing, implant materials, and dialysis membrane ${ }^{278,279}$. Nanofibers of PAN, having diameters in the range from $100 \mathrm{~nm}$ to a few microns, could be easily produced via the electrospinning technology $y^{280,281}$. Due to the high specific surface areas, PAN nanofibers were used in tissue engineering as scaffolds ${ }^{282-284}$. Furthermore, the high crystalline melting point $\left(317^{\circ} \mathrm{C}\right)$ of PAN, its limited solubility in certain solvents, and the superior mechanical properties of its fibers ${ }^{279}$ make it potential to build a stable 3D 
scaffold under mechanical loading. Because the Core-Sheath structure of nanofiber could result to better mechanical properties ${ }^{285}$, we coated a layer of PPy to improve mechanical properties of PAN nanofibers. The PPy was chosen as the coating layer of the nanofibers because of its widespread use in numerous applications in tissue engineering owing to its biocompatibility, easy manipulation, good stability, and high electrical conductivity ${ }^{150,286-}$ ${ }^{288}$. Additionally, PAN nanofibers could be easily coated with polypyrrole (PPy) to induce a conductive layer ${ }^{288,289}$, which is promising to induce electrical stimulation to PAN nanofibers in future use.

In this study, we report a novel method for creating the 3D-NFs that could provide a $3 \mathrm{D}$ structure for 3D hMSC culture. Moreover, we show its enhanced mechanical property by coating polypyrrole. The results demonstrate that the 3D-NFs can promote convenient infiltration and adhesion of cells into the inner interwoven matrices of the scaffold. After coating conductive polymer layer, the sheath-core 3D NFs with super-elastic properties could support biomechanical stimulations of human mesenchymal stem cells (hMSCs). Under such dynamic culture conditions in 3D environments, collagen production of hMSCs was improved in their immediate microenvironments, which is also known to support cell adhesion and survival ${ }^{239}$. However, the proliferation was maintained under such dynamic condition.

\subsection{Materials and Method}

\subsubsection{Fabrication of Scaffolds (2D-, 3D-NFs, and 3D-CNFs)}

For NFs fabrication, a precursor solution was prepared by dissolving PAN ( $\mathrm{Mn}=90,000)$ 
in the solvent of $\mathrm{N}, \mathrm{N}$-dimethylformamide (DMF) at the concentration of $10 \mathrm{wt} \%$. The prepolymer solution was constantly stirred to become viscous and homogeneous. The electrospinning was performed using a customized spinning system. Firstly, the mixed solution was fed into a syringe equipped with a needle (22 gauge, blunt tip). A syringe pump was used to press the syringe at a rate of $1.0 \mathrm{~mL} / \mathrm{h}$. The distance between the needle tip and the collector was $10 \mathrm{~cm}$. A high voltage DC power supply $(15 \mathrm{kV})$ was applied to generate a continuous jetting stream. The resultant nanofibers were deposited onto the collector directly and continuously. For 2D-NFs, a piece of Aluminum foil was utilized as the collector. For 3D-NFs, a borosilicate beaker $(1000 \mathrm{~mL})$ with $800 \mathrm{~mL}$ ethanol was used for nanofiber deposition and it was shaken every 5 mins during the electrospinning process. Eventually, the nanofiber dispersion was replaced by $800 \mathrm{~mL}$ DI water three times with rigorous shaking. The porosity was controlled by suspending the same weight of nanofibers into DI water with different volumes. The final porosity was controlled by achieving a $0.125 \mathrm{mg} / \mathrm{mL}$ Nanofiber_Mass/Water_Volume $(\mathrm{M} / \mathrm{V})$. The nanofibers entangled together when they were injected into the ethanol solution and shaken in DI water. The as-prepared 3D-NFs with water was frozen at $-80{ }^{\circ} \mathrm{C}$ in a refrigerator for $24 \mathrm{~h}$ before freeze drying for $48 \mathrm{~h}$. The relevant temperature and humidity during the electrospinning were $20 \pm 3^{\circ} \mathrm{C}$ and $40 \pm 5 \%$, respectively. A humidity controller (Shiteng, China) was set to control the humidity to $40 \%$ with a $\pm 5 \%$ accuracy as instructed by the manufacturer. A high humidity in the electrospinning process could inhibit the evaporation of the organic solvent and be failed to form fibers. The fabrication steps of 2D- and 3D-NFs were shown in the Figure 4-1. 


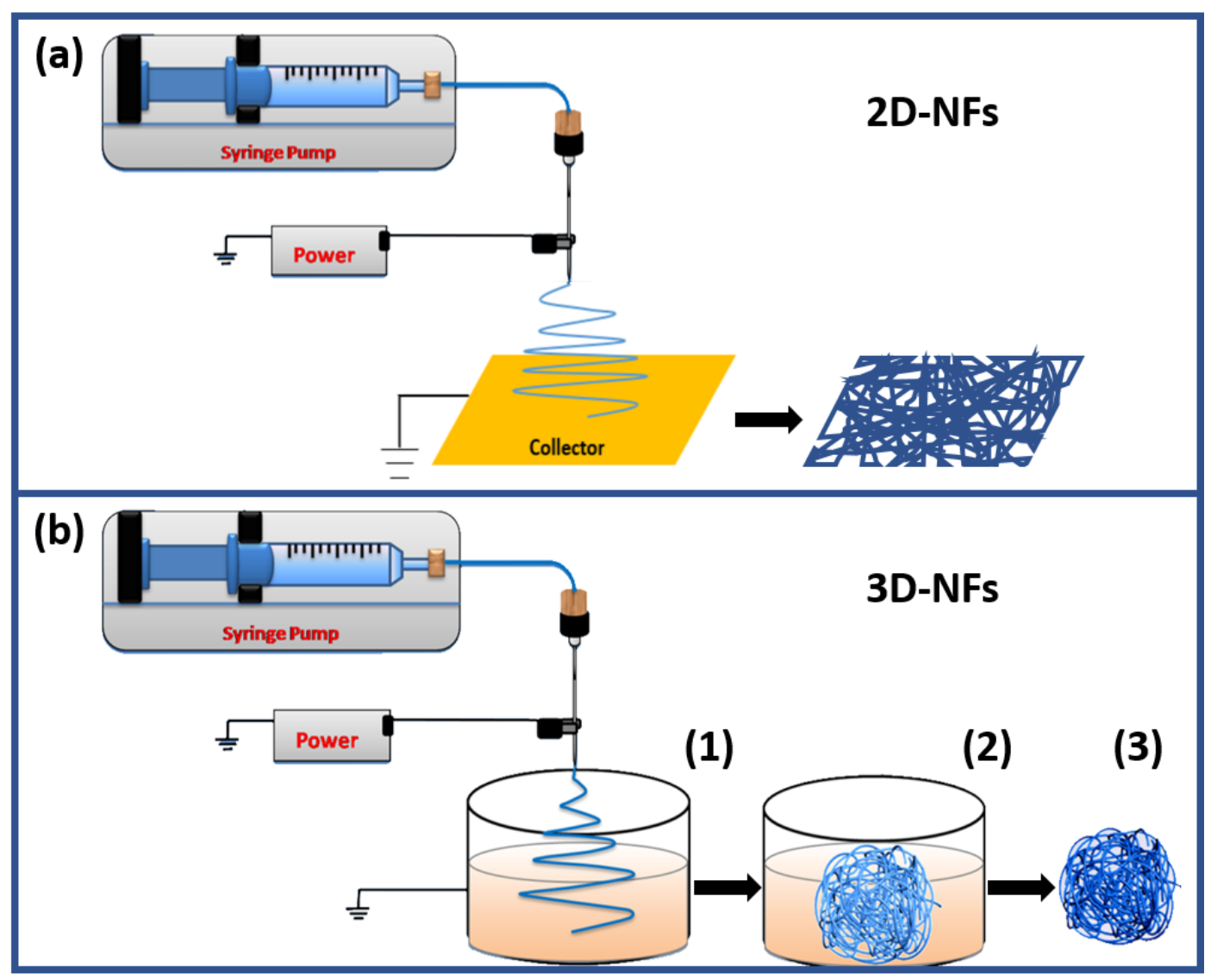

Figure 4-1 Schematic diagram of the fabrication steps for 2D-NFs (a) and 3D-NFs (b). (1)

Electrospinning nanofibers into ethanol solution. (2) The obtained mixed solution was replaced with DI water by adding DI water 3-5 times with rigorous shaking. (3) 3D-NFs prepared by freeze drying.

To further improve the functional properties of 3D-NFs, like conductivity and improved mechanical property, for the wider range of applications in tissue engineering, conductive polypyrrole (PPy) shell layer was coated on the surface of the nanofibers by in-situ surface polymerization of pyrrole using $\mathrm{FeCl}_{3}$ as the oxidant ${ }^{274}$. Briefly, the scaffold was placed in a $0.04 \mathrm{M}$ pyrrole aqueous solution. The polymerization of pyrrole and deposition of PPy were initiated by adding the same volume of $0.084 \mathrm{M} \mathrm{FeCl}_{3}$ aqueous solution at room temperature. The mixture was treated by an ultrasonic cleaner for $1 \mathrm{~h}$. The scaffold was 
stained to black as a result of the deposition of black PPy. Then, the 3D-CNFs were washed by chloroform and freeze dried with water to achieve desired $\mathrm{M} / \mathrm{V}$. The photograph of 3D-CNFs and 3D-NFs was shown in Figure 4-2. All the nanofibers were utilized without any fusion, physical or chemical bonding, and any chemical treatment. The nanofibers randomly entangled during the fabrication process.

\section{D-CNFs (PPy Coated) 3D-NFs}

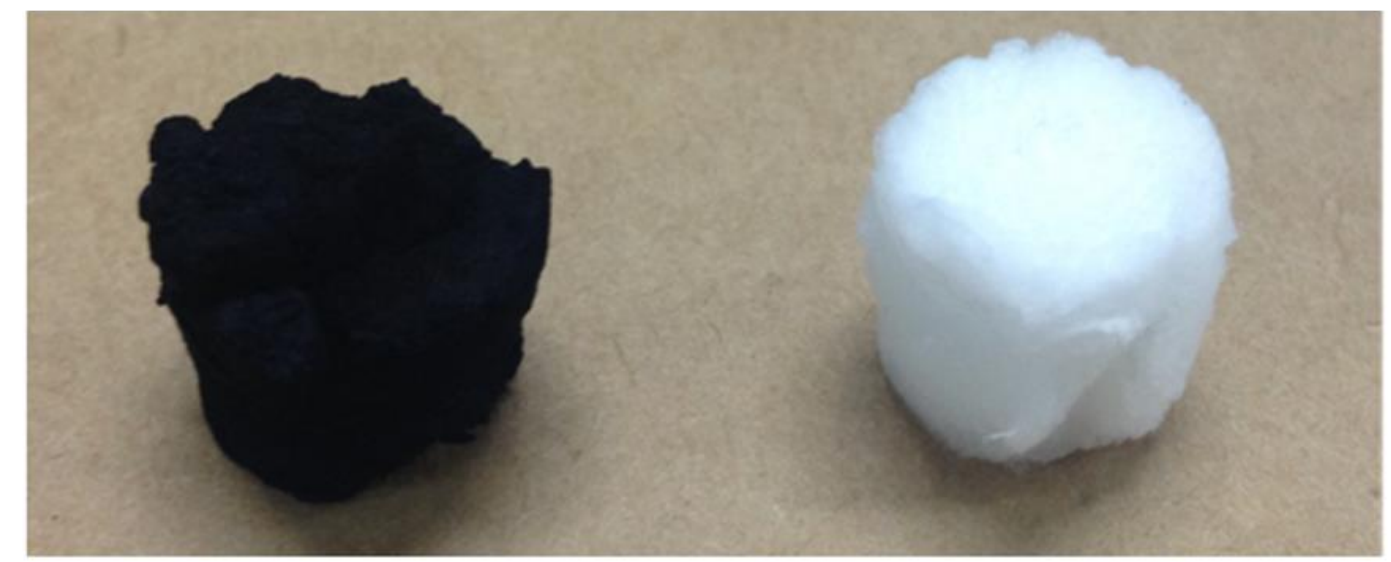

Figure 4-2 Optical photograph of freeze dried 3D-CNFs and 3D-NFs.

\subsubsection{Culture of hMSCs}

hMSCs derived from human bone marrow was obtained from Life Technologies, Singapore. MSCs of passage 2-4 were used here. 2D-NFs were cut and fitted into 24-well plates with a diameter of $15 \mathrm{~mm}$, and then sterilized using $70 \%$ ethanol aqueous solution for 12 hours, followed by washing with $1 \mathrm{X}$ PBS for three times. 3D-NFs and 3D-CNFs were cut in 24-well plates and fitted into the wells by soaking in DI water followed by freeze drying method to achieve $0.125 \mathrm{mg} / \mathrm{ml} \mathrm{M} / \mathrm{V}$. The sample is a cylinder with a diameter of $15 \mathrm{~mm}$ and a height of $5 \mathrm{~mm}$. All the samples were sterilized with $70 \%$ ethanol for 12 hours. The samples were washed with sterile PBS and exposed to UV 
generated by biological safety cabinet (BSC) (Thermo Scientific 1376 A2) for $2 \mathrm{~h}$ before seeding. Before seeding, the samples were pretreated by incubated in the FBS supplemented DMEM growth medium for 1 hour. hMSCs were seeded onto or into the various substrates at a certain cell density $\left(1.2 \times 10^{5}\right.$ cells/well $)$ with $1 \mathrm{~mL}$ DMEM supplemented with FBS and penicillin/streptomycin as mentioned in Chapter 3. All the cell constructs were incubated in a humidified condition with $5 \% \mathrm{CO}_{2}$ at $37^{\circ} \mathrm{C}$.

\subsubsection{Fluorescent and SEM Imaging}

To assess cell morphology, the cells were fixed with $3.7 \%$ paraformaldehyde (Sigma, Singapore) for $30 \mathrm{~min}$ and washed with $1 \times$ PBS. Cells were treated with $0.1 \%$ Triton X-100 (Sigma, Singapore) for $15 \mathrm{~min}$ and washed with $1 \times$ PBS. Following this, the samples were incubated with rhodamine-conjugated phalloidin (Life Technologies, Singapore) for $60 \mathrm{~min}$ at 1:500 dilution at room temperature. The cells were then washed with PBS and incubated with DAPI (Life Technologies, Singapore) at 1:1000 dilution for 5 min. Rinsed samples were imaged with an inverted confocal microscope.

To further analyze cellular morphology in the scaffolds, cells were fixed in $4 \%$ formaldehyde solution for $1 \mathrm{~h}$ and followed by washing with DI water for 3 times. Cells in DI water were frozen overnight and dehydrated with a freeze dryer. Cells were then imaged with the SEM (Hitachi S4800, Japan).

\subsubsection{Dynamic Loading}

Initial dynamic loading started on the day after 24-hour cell culture. A dynamic 
compression setup was constructed based on a shaker (V4, Gearing \& Watson) mounted with some aluminum rods with a diameter of $12 \mathrm{~mm}$, as shown in Figure 4-3. The shaker could provide a sinusoidal dynamic compression with adjustable frequency and amplitude. Cell-laden scaffolds were stored in 24 -well plate without bonding to the bottom. 3D scaffolds were cylinders fitted into 24-well plates with a height of $5 \mathrm{~mm}$. The M/V was controlled as $0.125 \mathrm{mg} / \mathrm{ml}$. In each experiment, 3 samples were compressed at the same time. The compressive rods first contacted to the top surface of the cell-laden scaffolds, and the scaffold touched the bottom of the wells without deformation. The amplitude and the frequency of rods' movement was adjusted. The loading protocol consisted of a $20 \%$ dynamic strain on the scaffolds at a frequency of $2 \mathrm{HZ}$, which is within the physiological range of strain that native cartilage tissues were subjected ${ }^{290}$; loading was carried out for 2 hours per day continuously in the incubator $\left(5 \% \mathrm{CO} 2\right.$ and $\left.37^{\circ} \mathrm{C}\right)$ and 5 days per week for 2 weeks with static and TCP controls cultured in parallel. Considering the elastic hysteresis (Figure 4-7 and Figure 4-8), which also happened in other mechanical stimulus bioreactors $^{291}$, the $20 \%$ strain consisted of a dynamic strain superimposed on a tare strain. The medium was fully changed twice a week. 


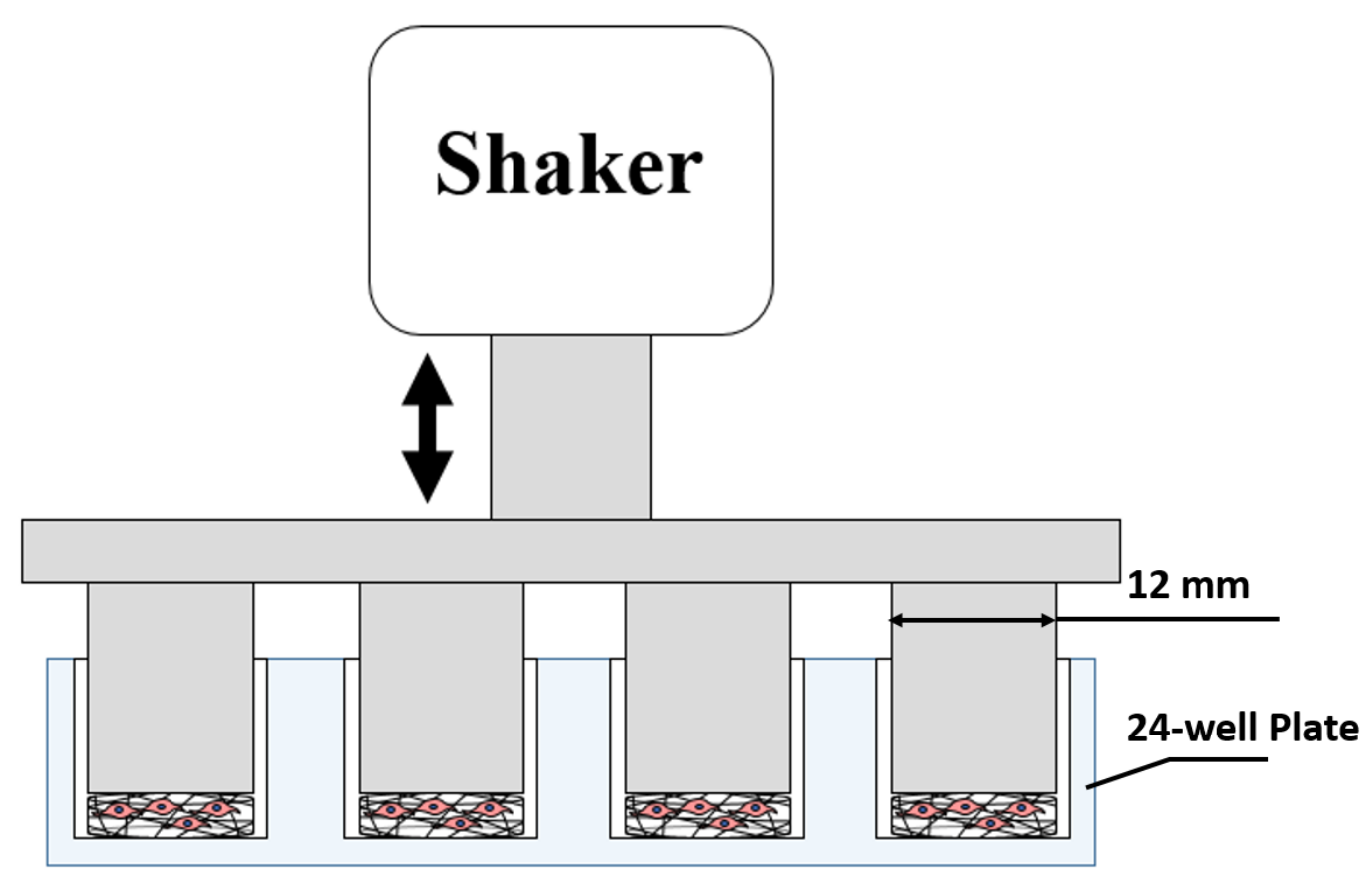

Figure 4-3 Schematic of a bioreactor for dynamic loading.

\subsubsection{Attachment and Proliferation}

Cellular attachment and proliferation (results in Section 4.4.2) were characterized at different time points. Briefly, Quant-iT PicoGreen dsDNA Assay Kit (Life Technologies, Singapore) was used to quantify the DNA content of the samples in which hMSCs were cultured for attachment comparison. On certain culture days, samples were transferred from the culture wells to a new 24-well plate to exclude the cells dropped to the bottom of the culture well, and incubated with lysis buffer $(0.2 \%$ Triton $\mathrm{X}-100)$ followed with several freeze-thaw cycles. The sample was then prepared as manufacturer's instructions. Fluorescence of the samples was measured at 485/535 nm using a Multiskan Spectrum microplate reader (Thermo Scientific, Singapore). The cell number were converted from DNA values and plotted. The results were expressed as cell number (using a standard DNA curve created as manufacturer's instruction and DNA extracted from known 
numbers of MSCs) divided by the initial seeding number of cells $\left(1.2 \times 10^{5}\right.$ cells/well). The proliferation of cells (in Section 4.4.4) was characterized by PrestoBlue cell viability kit (Life Technologies, Singapore). Briefly, the cell-laden scaffolds and TCPs were washed and incubated in medium containing 10\% PrestoBlue reagent for $1 \mathrm{~h}$ in a humidified atmosphere at $37{ }^{\circ} \mathrm{C}$ and $5 \% \mathrm{CO}_{2}$. Culture medium containing $10 \%$ PrestoBlue reagent was incubated with no cells and served as the blank control. The absorbance of the reduced PrestoBlue reagent was measured at $570 \mathrm{~nm}$ and $600 \mathrm{~nm}$ as the reference with a Multiskan Spectrum microplate reader (Thermo Scientific, Singapore). The absorbance reading, normalized to TCP controls, was utilized to represent the cell number as the manufacturer's protocol.

\subsubsection{Collagen Evaluation}

Cellular collagen content was quantified on day 14 according to the previously reported method $^{292}$. Briefly, The cell-nanofiber constructs were washed with sterilized PBS and then incubated using papain digestion buffer $(125 \mu \mathrm{g} / \mathrm{mL}$ Papain, $5 \mathrm{mM}$ L-cysteine, $5 \mathrm{mM}$ EDTA and $\left.100 \mathrm{mM} \mathrm{Ha} 2 \mathrm{HPO}_{4} \mathrm{PH}=7.5\right)$ at $70^{\circ} \mathrm{C}$ for $16 \mathrm{~h}$. After incubation, constructs were spun down, and supernatants were collected and used to hydroxyproline content evaluation. Hydroxyproline content was measured on the papain-digested supernatant using (Hydroxyproline Assay Kit, Sigma, Singapore) according to manufacturer's instructions. Deionized (DI) water was used as a blank in the assay measurements. 


\subsubsection{Characterization of Osteogenic Specification}

To prepare the Positive Control Groups, we cultured cells on 3D-CNFs with the same density before osteogenesis. Induction of osteogenic differentiation in MSCs was initiated by introducing the osteogenic medium, comprising low glucose DMEM supplemented with $10 \%$ FBS, $50 \mu \mathrm{g} / \mathrm{ml}$ ascorbic acid, $1 \mathrm{mM}$ sodium pyruvate, $100 \mathrm{U} / 100 \mu \mathrm{g}$ penicillin/streptomycin, $1 \times$ glutamax, $10 \mathrm{mM} \quad \beta$-glycerophosphate, and $10^{-7} \mathrm{M}$ dexamethasone ${ }^{230}$, on day 1 (the day after seeding). The cells were then cultured (with or without dynamic loading) in a humidified atmosphere with $5 \% \quad \mathrm{CO}_{2}$, and the differentiation medium was changed twice per week.

On day 14, cells were fixed in the $10 \%$ neutral buffered formalin solution (Sigma, Singapore) for $20 \mathrm{~min}$ and treated with $0.1 \%$ Triton X-100 (Sigma, Singapore) for $5 \mathrm{~min}$ followed by PBS wash for two times. Then cells were incubated with 1\% BSA blocking buffer for 1 hour at room temperature. The cells were then incubated with primary antibody, anti-RUNX2 antibody (Millipore, Singapore), in 1:1000 dilution for overnight at $4{ }^{\circ} \mathrm{C}$ followed by three times PBS wash. Cells were then incubated with Alexa Fluor ${ }^{\circledR} 488$ secondary antibody (Millipore, Singapore) at 1:1000 dilution at room temperature for 1 hour followed by DAPI staining. 


\subsection{Results and Discussion}

\subsubsection{Structural Characterization of 2D- and 3D-NFs}
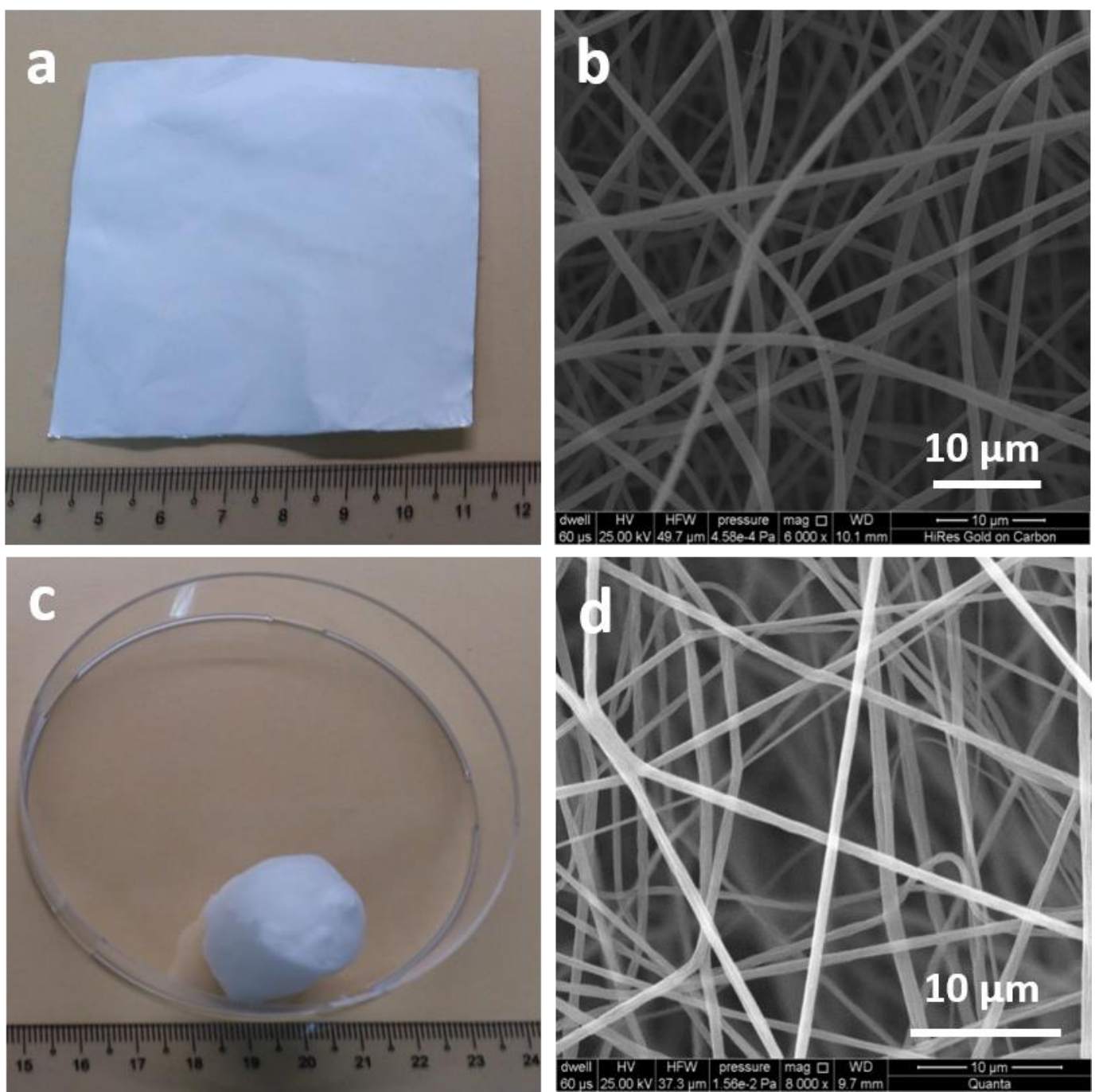

Figure 4-4 Optical photographs and SEM images of 2D-NFs collected from the Aluminum foil (a-b), and 3D-NFs $(0.5 \mathrm{mg} / \mathrm{mL})$ collected in the ethanol (c-d).

Here, the samples collected from Al foil were defined as 2D samples, while samples collected from the ethanol solution were called 3D samples. The key challenge in preparing the $3 \mathrm{D}$ NFs is the formation of interconnected porous matrix composed of nanofibers. In this study, 3D NFs were designed to have an open and continuous pore 
structure. The porosity and pore size were controlled by improved electrospinning and collection system and structure reconstruction. The fabrication consists of three distinct steps: (1) Nanofibers with certain weight were collected and transferred to the certain volume of water; (2) Homogenization of nanofibers was conducted with an orbital shaker (Orbit 1000, Labnet) for overnight at a speed of 100 RPM to let the nanofibers fully extended, so the volume of the water equaled to the volume of the scaffolds in water; and (3) Freeze-drying assembly. As shown in the schematic diagram of the fabrication process (Figure 4-1 (b)) polyacrylonitrile (PAN) nanofibers were directly spun into the ethanol solution to make the nanofibers fully extended. With a progressive increase in the electrospinning time, PAN nanofibers were produced and well-dispersed in the ethanol mixed solution. Eventually, 3D-NFs were obtained by freeze-drying method before which the nanofiber dispersion was replaced 3-5 times using deionization (DI) water along with shaking. Optical photographs of nanofibers fabricated with electrospining method coupled with different collectors were shown in Figure 4-4 (a) and (c). It clearly showed that nanofibers collected from the Aluminum foil were 2D non-woven meshes while nanofibers collected in ethanol had a 3D architecture. The nanofibers in 3D-NFs formed fibrous networks, and clearly exhibited disperse status, i.e., the 3D NFs have continuous and interconnected pores (Figure 4-4 (d)). However, the nanofibers in the 2D-NFs (Figure 4-4 (b)) appeared closely packed together. These results indicated that the obtained 3D-NFs could successfully overcome the structural and functional limitations of the $2 \mathrm{D}$ fibrous mats.

To obtain 3D NFs with the desired porosity and pore size, to meet the specific 
requirements of different applications, we homogenized the as-prepared 3D-NFs in DI water with specific concentration and followed by freeze drying. Since the samples were fully expended in the water, the dried samples' volumes corresponded to the volume of water used, the porosity of the scaffolds can be controlled by adjusting the ratio of Nanofiber_Mass/Water_Volume (M/V) as shown in Figure 4-5. The samples with same $\mathrm{M} / \mathrm{V}$ would have the same porosity, even considering the shrinkage in the freeze drying. Three arbitrarily values of $\mathrm{M} / \mathrm{V}, 0.5,0.25$ and $0.125 \mathrm{mg} / \mathrm{mL}$, were selected and named as samples 1-3 (S1-3) to show low, medium and high porosities, respectively. The SEM images (Figure 4-5 (b)), clearly indicated that the M/V significantly affects the internal structure and organization of the 3D-NFs, i.e., as $\mathrm{M} / \mathrm{V}$ decreases, the pore size and porosity increase. From the above results, it could be concluded that the required internal structure could be controlled by the proposed preparation process. The prepared 3D-NFs showed large enough interconnected pores to promote diffusional penetration of cells into the interior of the 3D nanofibrous scaffold without the need for any external force. Thus, 3D-NFs could attract, retain and grow cells within the 3D ECM-like microenvironment and with enhanced deep cellular infiltration, cellular growth, proliferation, and differentiation. Additionally, compared to the 2D NFMs, 3D NFs allow easy and efficient transportation and exchange of nutrients required for cell growth and metabolism to the interior regions of the scaffolds. Subsequently, the fibrous matrix could also facilitate the discharge of the produced waste of cells to outside medium. These processes are crucial for large scale proliferation of hMSCs as well as for the formation of specifically engineered tissue into the desired lineage. 


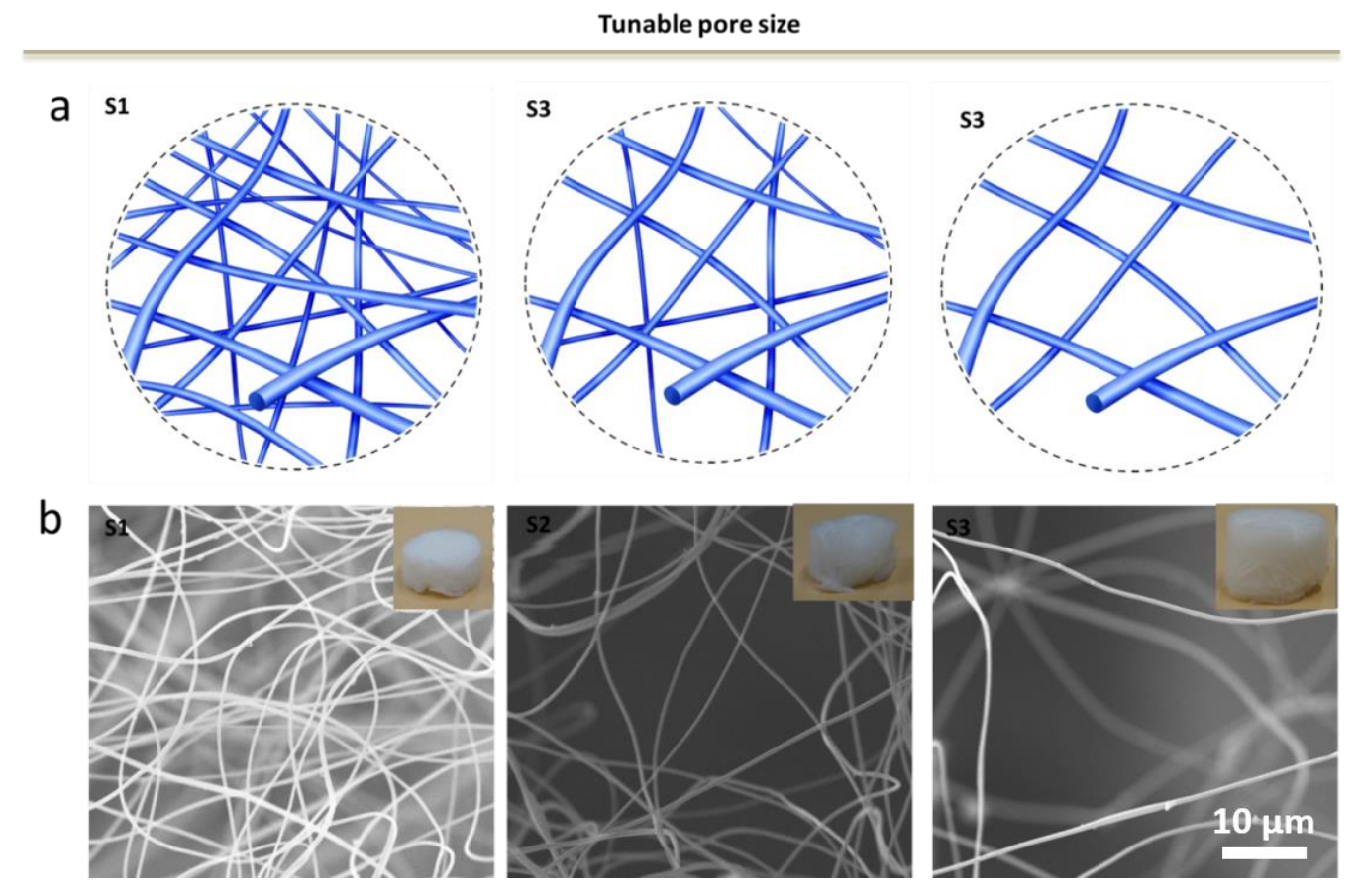

Figure 4-5 (a) Schematic description of the changes in the internal micro-porous structure with varying nanofiber M/V. (b) The microscopic architecture of the 3D NFs at various densities, they are named sample $1(\mathrm{~S} 1,0.5 \mathrm{mg} / \mathrm{mL})$, sample $2(\mathrm{~S} 2,0.25 \mathrm{mg} / \mathrm{mL})$ and sample $3(\mathrm{~S} 3,0.125 \mathrm{mg} / \mathrm{mL})$. The insets are their optical photographs. Scale bar: $10 \mu \mathrm{m}$.

The volume change of scaffolds during freeze drying and rehydration was not obvious and not quantified. We controlled the initial $\mathrm{M} / \mathrm{V}$ or density before the freeze drying. For samples used in cell culture, the initial $\mathrm{M} / \mathrm{V}$ and the dimension were controlled. We assumed the $\mathrm{M} / \mathrm{V}$ of each sample would be as same as others' during the cell culture.

\subsection{2 hMSC Cultured in 2D- and 3D-NFs}

In order to gain insight into the cells' growth and morphology in 3D-NFs, the cellular responses of 3D-NFs were characterized using fluorescent images of cells through a confocal microscope. The cell density is $1.2 \times 10^{5}$ cells/well in a $24-w e l l$ plates. The F-actin staining of the hMSCs show that the hMSCs in 3D-CNFs (Figure 4-6 (c)) were not 
completely spread out with a flat morphology as often seen in 2D cultures, and are often found hanging on to multiple nanofibers in their immediate vicinity. In contrast, the cells cultured on 2D-NFs (Figure 4-6 (b)), resembled 2D cell cultures (similar to TCPs (Figure 4-6 (a))) with extended cell area which could be attributed to the cell retention at the 2D surface due their inability to penetrate into the bulk of nanofibrous mesh. However, F-actin of cells on the TCPs showed all well-spread morphology with higher cell area due to the isotropic surface of the TCPs which allows hMSCs to spread freely in every direction (Figure 4-6 (a)) as commonly shown in standard conventional TCP cultures. These observations from the fluorescent images corresponded well with the morphology of the cells (Figure 4-6 (d)-(f)) and demonstrate that the 3D-NFs allow adequate penetration of cells into the interior regions of the scaffold and promote their growth in a 3D microenvironment. The attachment after the first 6 hours was shown in Figure 4-6 (g). The cell attachment in TCPs, 2D-NFs, and 3D-NFs was 94\%, 93\%, and 98\% respectively. The attachment here meant the cells could attach to the 3D-scaffold without falling from the nanofiber. This indicated the cell attachment in the 3D-NFs was slightly better than that in TCPs and 2D-NFs. In terms of cell proliferation, it was clearly seen that the number of hMSCs in each sample have rapidly increased after 3 days as shown in Figure 4-6 (h). However, the number of hMSCs in 3D-NFs was significantly higher than that in TCPs and 2D-NFs at each time point, which may be attributed to the porous $3 \mathrm{D}$ architecture. The 3D structure and higher surface area of the 3D scaffold could support higher cell density maintaining the proliferation while the cells on 2D surface (both TCP and 2D-NFs) had a lower proliferation rate due to the high density ${ }^{293,294}$. Interconnected pores could provide 
enough space for adequate cell penetration and proliferation.
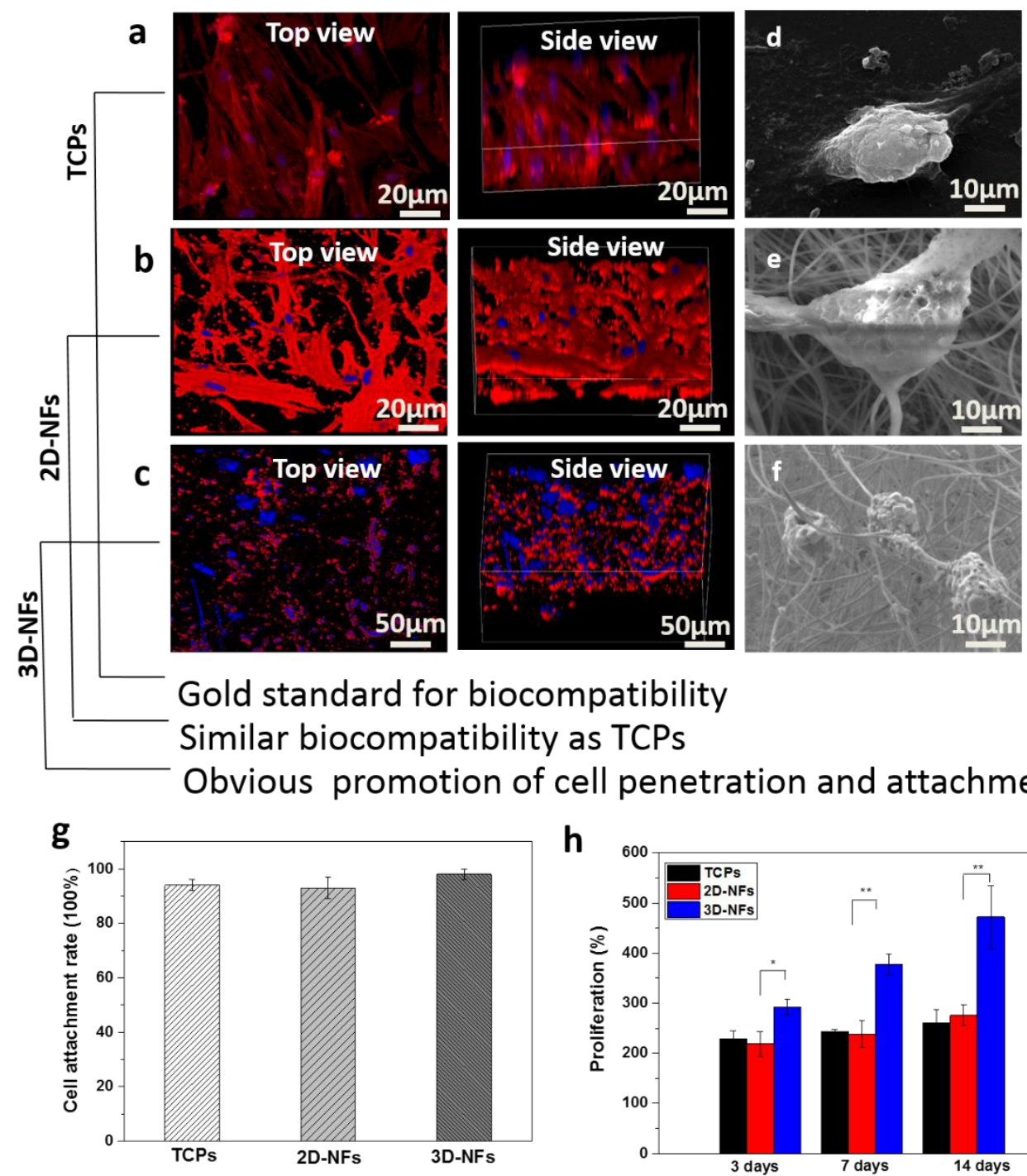

Side view

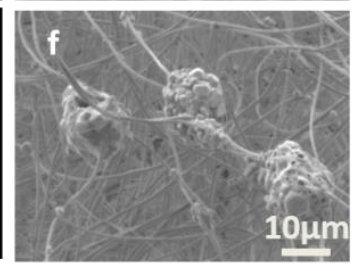

$50 \mu \mathrm{m}$

Lin

mpatibility

Similar biocompatibility as TCPs

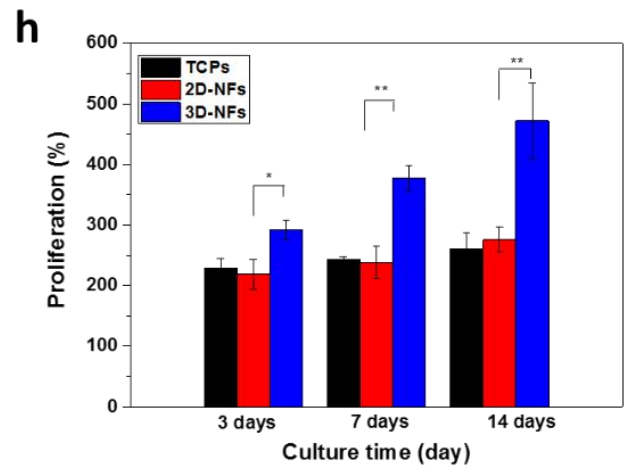

Figure 4-6 (a-c) Fluorescent images of hMSCs on day 5 on TCPs, 2D-NFs, and 3D-NFs.

(d-f) SEM images of hMSCs on day 5 on TCPs, 2D-NFs and 3D-NFs. (g) The attachment and (h) proliferation of hMSCs cultured for certain days on the TCPs, 2D-NFs and

3D-NFs. Proliferation values were normalized by the number of cells after one-day culture.

Data are expressed as mean $\pm \mathrm{SD}(\mathrm{n}=3) ; *$ indicated $p<0.05$ and $* *$ meant $p<0.01$. 


\subsubsection{Mechanical Property of 3D-CNFs}

Compared to the original 3D nanofibers, the obtained 3D-CNFs retained the porous structure, interwoven architecture (Figure 4-7 (a)). The coated PPy layer could be seen clearly by TEM and high magnification SEM image (Figure 4-7 (b) (c) and (d)). As measured from the TEM images, the core-sheath nanofibers had a diameter around 500 $\mathrm{nm}$. The thickness of the outer PPy coating layer was around 70-80 nm while the core PAN nanofiber had a diameter around $300-400 \mathrm{~nm}$.

More importantly, the PPy-coated nanofibers scaffolds had a good performance in recovery under the compression. Although 3D-NFs allowed cells to migrate inside, the mechanical properties were not good enough. After one-time compression to $40 \%$ strain and released to unloaded state, the 3D-NFs cylinder only recovered to $78 \%$ of its original height while 3D-CNFs had a 100\% recovery (not shown here). It could be attributed to the core-sheath structure ${ }^{285}$. The compression stress-strain curves and photos of the CNFs are shown (Figure 4-7 (e) and (f)) in air and water respectively. The cylinder-shape samples with a diameter of $15 \mathrm{~mm}$ and height of $10 \mathrm{~mm}$ were used in the compressive test with the Instron 5566. The samples were compressed and released at a rate of $\sim 0.1 \% / \mathrm{s}$ from $0 \%-$ $40 \%$ strain. The 3D-CNFs showed linear elasticity at low strains of less than $10 \%$ in air. After a yielding point, an increase was observed in stress for response to a large deformation, or energy absorption. The CNFs were released after $40 \%$ compressive strain, during which the stress is $4.02 \mathrm{KPa}$ (in the air) and $1.2 \mathrm{KPa}$ (in DI water), respectively. The decrease of strength in the wet condition was reported by many studies ${ }^{295-297}$. It could be explained that water worked as a plasticizer that swells and softens materials. Despite 
the relative decrease of compression stress in the water compared to that in air, the 3D-CNFs could be well restored to its original state as it in the air.

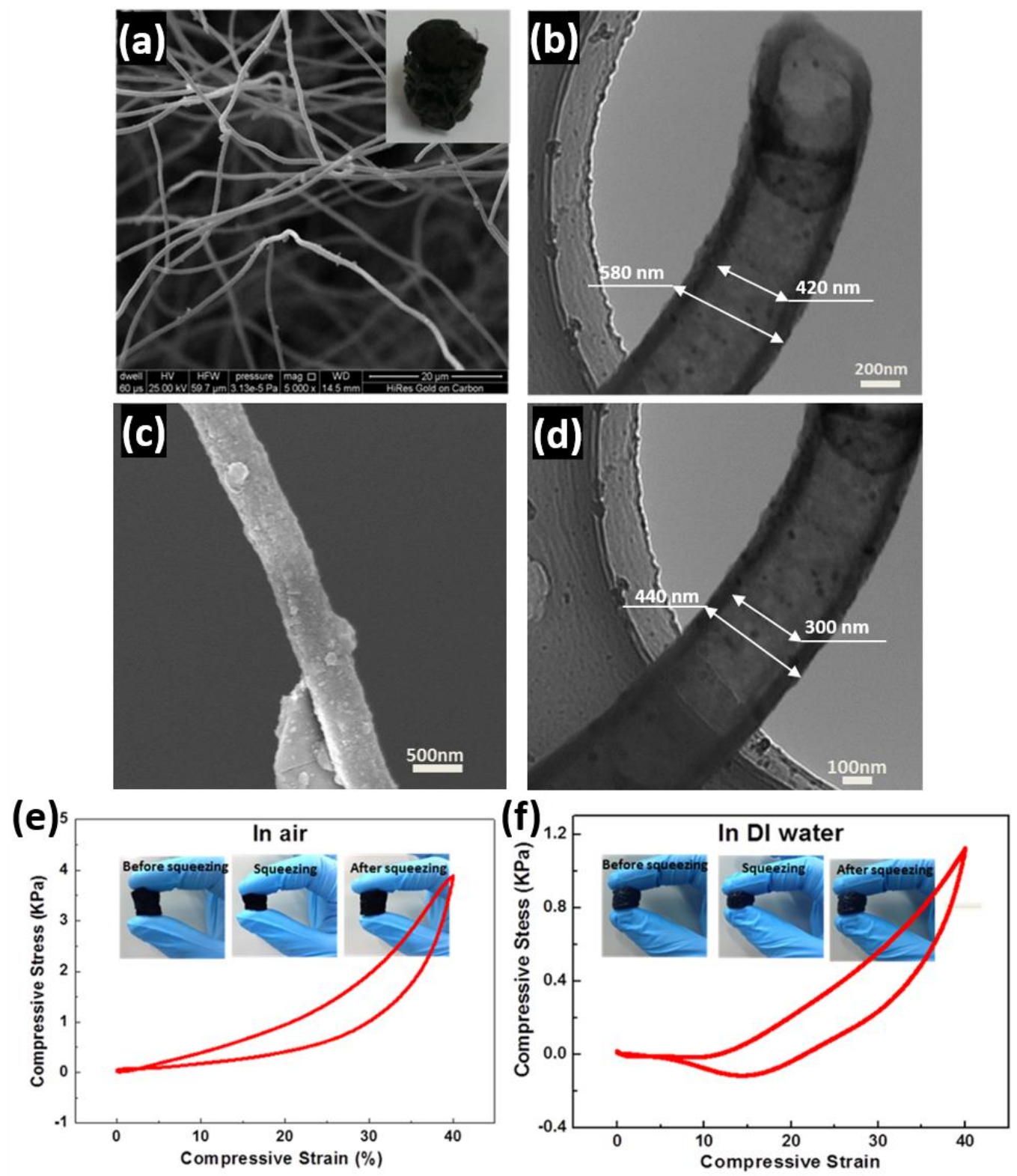

Figure 4-7 (a) (c) SEM image of 3D-CNFs (the inset is the optical photograph of a 3D-CNFs block). (b) (d) TEM image of single core-shell PPy nanofiber. (e)(f)

Compressive strain-stress curve and optical picture of 3D-CNFs before and after compression in the air and DI water, respectively.

After ten cycles in the air, the CNFs still recovered well, close to its original state as 
shown in Figure 4-8, which may be attributed to the combined effects of the unique "core-shell" structure and PPy layer along the nanofibers which could have played a combined role in enhancing the fiber strength. On the other hand, this synergistic effect also makes CNFs adjust to absorption of the external force. These results demonstrated that the 3D-CNFs not only allow for easy penetration, attachment and proliferation of cells, but can also provide mechanically viable 3D nanofibrous micro-environment with appropriate flexibility to enhance cell biological response and functional expression under dynamic culture condition.

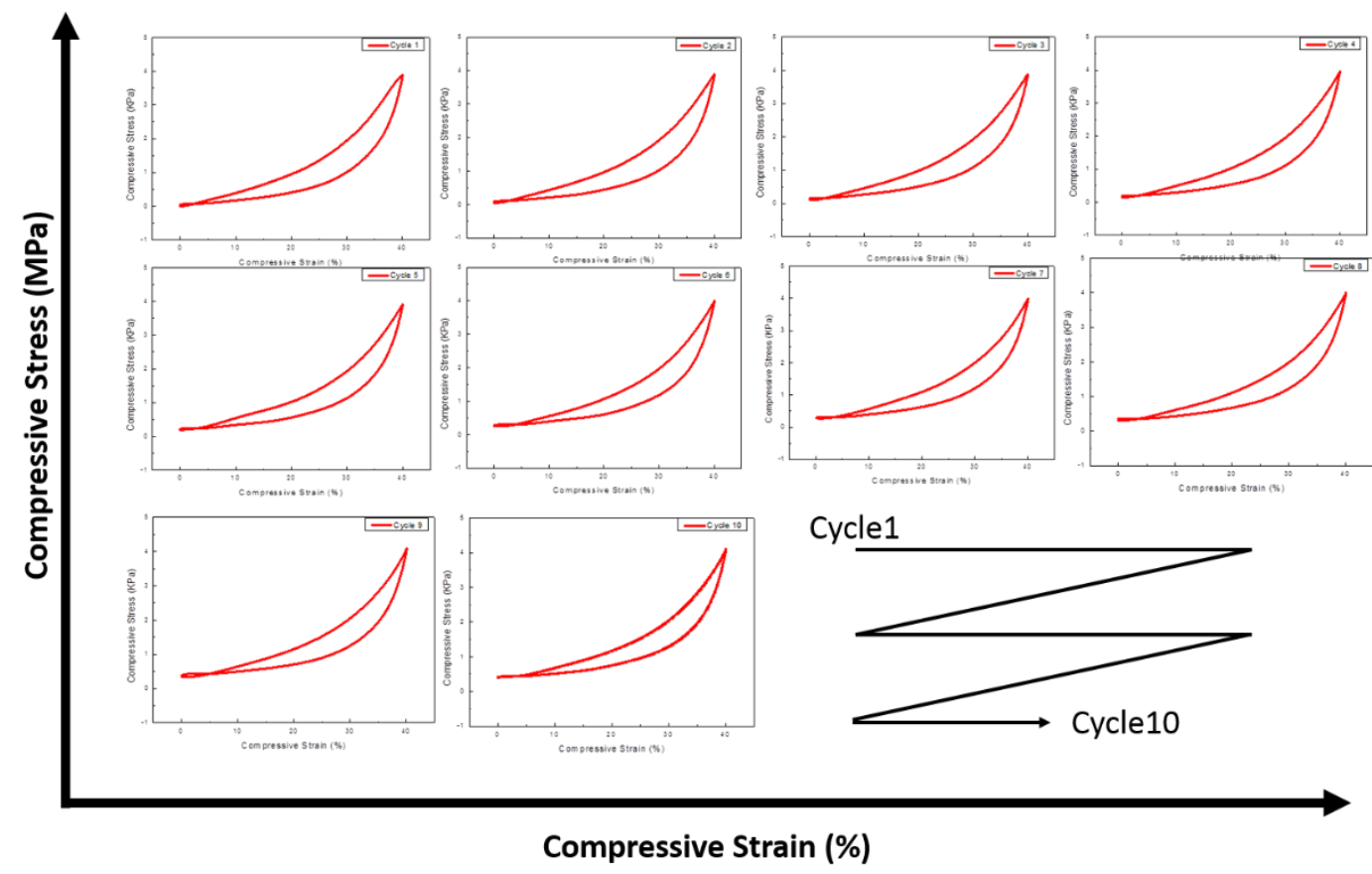

Figure 4-8 The compressive strain-stress curves of 3D-CNFs during ten cycles in the air.

The axis range of compressive strain in the figure is from $-5-40 \%$, and the axis range of compressive stress in the figure is from -1-5 MPa.

\subsubsection{Cell Response to Dynamic Loading}

Due to the good performance in compression test and a similar 3D architecture, 3D-CNFs 
were utilized to investigate the influence of dynamic loading on 3D cultured cells.

To explore in vitro response of hMSCs in 3D culture to the mechanical stimulation, hMSCs with a density of $1.2 \times 10^{5} /$ well were seeded in the 3D-CNFs. The cellular responses of dynamic compressive loading on hMSCs-laden 3D-CNFs were evaluated by fluorescence images of cells as shown in Figure 4-9. Cells were cultured for a long-term period ( 2 weeks) with or without dynamic loading as mentioned above. When the cells were grown under dynamic culture conditions, the F-actin and nuclei staining reveal the cells spread well inside the scaffold along the nanofibers (Figure 4-9 (a-c)), within distinct directions, which may be attributed to the continuous mechanical stimulations which promoted most cells to attach and grow on specialized nanofibers while adapting themselves to dynamic environment. The confocal images of F-actin stained hMSCs under the unstimulated/static state (without mechanical loading) showed uniformly spread cell distribution with a slight low level of cell-cell contact and spreading. The 3D images (Figure 4-9 (g) and (h)) showed cells distributed in a 3D space which confirmed the capability of 3D-CNFs in 3D culture. From the above results, we propose that the dynamic condition could make cells grow and survive along the specialized directions on the nanofibers. 


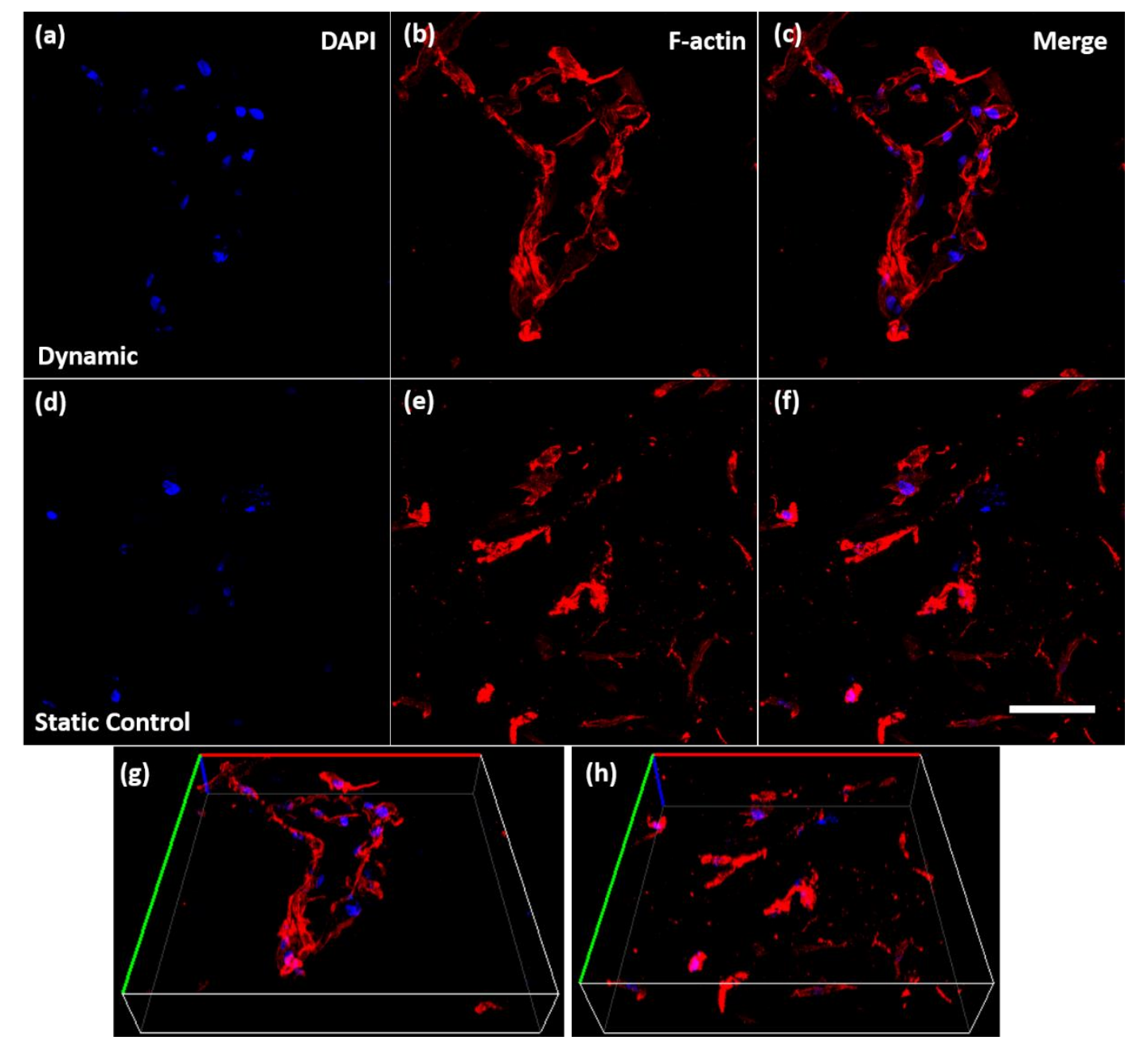

Figure 4-9 Fluorescence images of hMSCs cultured for 14 days in 3D-CNF scaffolds in dynamic (a-c) and static states (d-f), the bars are $100 \mu \mathrm{m}$.

The proliferation of hMSCs cultured on TCP, in 3D-CNFs with Dynamic loading, and in 3D-CNFs without dynamic loading (as static control) was evaluated by the PrestoBlue.

After 2 weeks of cyclic conditioning, no obvious difference was observed in cell amount between the loaded and the unloaded 3D-CNFs (Figure 4-10(a)), but both of these groups show significantly higher cell proliferation compared to the TCP cultures. However, after 2 weeks, the stimulated hMSC cultured 3D-CNFs showed higher collagen content compared to the unstimulated controls (Figure 4-10(b)). The results suggested that the effect of dynamic loading on the proliferation of hMSCs grown in the 3D-CNFs is 
negligible, although a slight drop of proliferation of cells cultured in dynamic loaded 3D-CNFs compared to that in static 3D-CNFs could be seen. However, the mechanical loading applied on 3D-CNFs could positively impact the collagen expression in hMSCs cultured in 3D-CNFs.
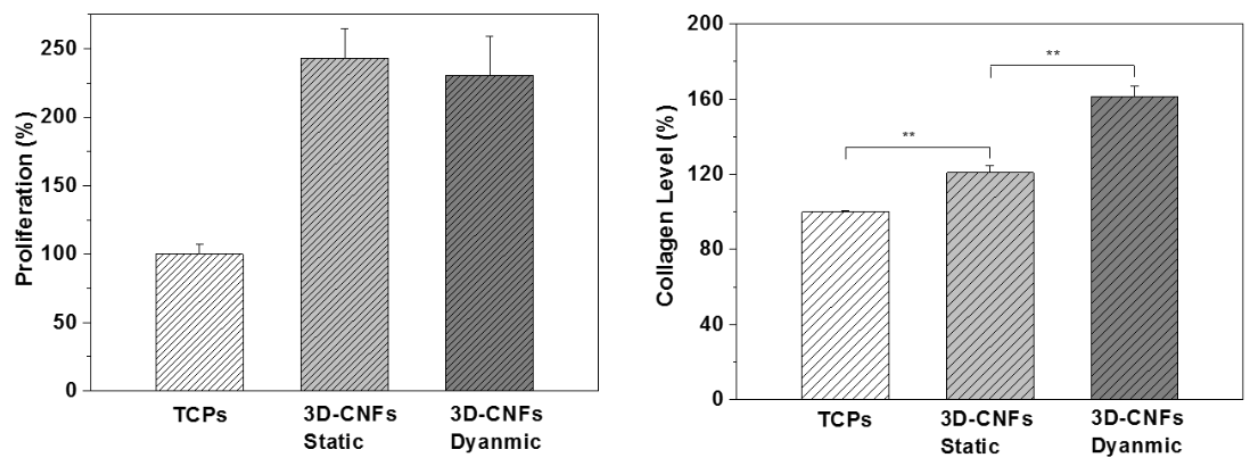

Figure 4-10 The proliferation of hMSCs cultured for 14 days onto/into TCPs and the 3D-NFs in static and dynamic states. (e) Collagen expression levels of hMSCs cultured 14 days onto/into the TCPs, 3D-NFs with the static and dynamic state. $* *$ indicated $p<0.01$. Previous studies had found the cyclic mechanical strain applied to cultured MSCs induces endogenous synthesis of potent growth factors that regulate lineage specification ${ }^{290,298,299}$. For example, the cyclic tensile could increase osteogenic growth factor response ${ }^{298}$ and upregulate the transcription of RUNX2 $2^{300}$. As nanofibers in our 3D-CNFs scaffolds would stretch when a uniaxial compression was applied ${ }^{301}$, we hypothesized that the osteogenic lineage happened in our experiment. Anti-RUNX2 antibody, a common osteogenic biomarker, was utilized for immunocytochemistry staining. After staining cells on Day 14, however, there was no RUNX2 expression in neither cells cultured in 3D-CNFs with dynamic loading nor cells cultured in static 3D-CNFs as shown in Figure 4-11. The positive control groups, no matter they were cultured with dynamic loading or not, showed 
RUNX2 expression obviously. There is no evidence that the osteogenic differentiation happened in either dynamic or static 3D-CNFs. However, the effect of mechanical loading on the osteogenic differentiation of MSCs was influenced by various parameters, for example, researchers found intermittent tension ((stimulation with stress applied for several minutes or hours each day) usually promote osteo-differentiation while continuous stimulation can inhibit the RUNX2 expression, an important biomarker for osteo-differentiation $^{302}$. However, in another study, the continuous mechanical tension has also been reported to promote oseto-differentiation ${ }^{300}$. The clear boundary to determine what kind of the mechanical loading is "good" for osteo-differentiation is still being explored. In this study, we found an intermittent mechanical loading applied on 3D nanofibrous scaffold would influence the collagen production without a clue of osteo-differentiation, which offered an alternative method to influence collagen production. 


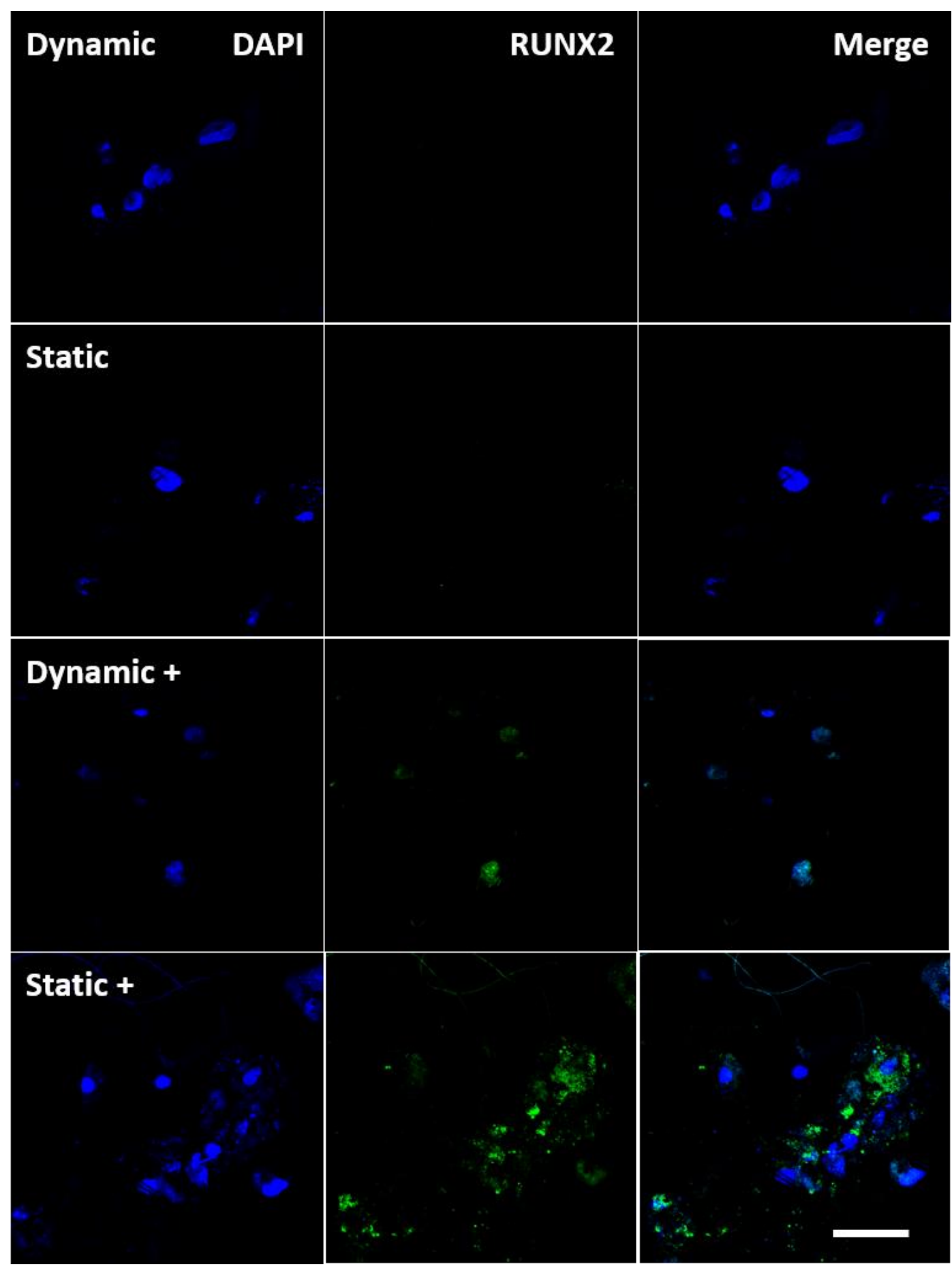

Figure 4-11 RUNX2 expression of MSCs after 14-day culture in both dynamic and static condition, as well as positive controls marked with "+". Scale bar: $50 \mu \mathrm{m}$.

\subsection{Conclusions}

It was demonstrated that 3D-NFs could provide an excellent 3D microenvironment 
favorable for cell penetration, adhesion, and proliferation. Thus, the scaffolds could be used for large-scale in vitro cell proliferation, rapid and controlled repair of damaged tissues. Furthermore, 3D-CNFs could provide optimal mechanical strength for the development and maintenance of load-bearing tissues as demonstrated by the cell response to cyclic dynamic tensile stimulation on 3D nanofibrous constructs.

Additionally, cell responses to dynamic loading via the 3D-CNFs were studied. It has indicated the mechanical stimulation via nanofibers helps cells grow along the fibers, with attachment/proliferation maintained. It also demonstrated the collagen expression of hMSCs in our 3D nanofibrous scaffolds could be improved by dynamic loading. The compression involved upregulation of collagen production of MSCs was also reported in the previous work ${ }^{64}$. However, different scaffolds and loading method were used in that work, and thus the discovery in our study offered an alternative way to regulate collagen production of MSCs. Furthermore, the collagen expression regulation was not related to osteogenic lineage speciation, and the mechanism of these phenomena needs further study. However, we provided experimental evidence to suggest that hMSCs culture in 3D-CNFs could be a useful platform to regulate cell behaviors to meet different applications in tissue engineering. 


\section{Chapter 5. Electrical Stimulation of Cortical Cells in 3D Conductive Nanofibrous Scaffolds}

\subsection{Significance and Novelty}

It is well known that cellular behaviors are significantly affected by cellular microenvironment, including mechanical supports, electrical and chemical cues, etc. Three Dimensional conductive nanofibers (3D-CNFs) provide the capability to regulate cellular behaviors using mechanical, geometrical and electrical cues, which are especially important in neural tissue engineering. However, previous studies were focused on 2D mats, suffering from the lack of the inner porous structures for 3D culture, instead of a 3D scaffold. Although some 3D nanofibrous scaffolds have been used in electrical stimulation, they were fabricated by complex processing, like layer-by-layer assembling ${ }^{303}$, or carding ${ }^{304}$.

To the best of the author's knowledge, this study is the first time when the combined effect of 3D culture and electrical stimulation (ES) on cortical cell cultures was investigated in 3D PPy-coated PAN nanofibrous scaffolds. The 3D structures were made from wet electrospinning without further processing. In this study, conductive nanofibers with a 3D structure were successfully prepared for the cortical cell culture. The combined effects of 3D conductive nanofibers and ES on neurons and other cortical cells (mainly glial cells) were studied via analyzing the morphology, proliferation, and maturation. These combined effects indicated that ES and 3D-CNF have broad applications in neural engineering, such 
as implantation, biofunctional in vitro model, etc.

*: Cortical cells here were utilized to describe the mixture of cells obtained from rat cortices. It contains neurons $\left(85.2 \pm 8.1 \%\right.$ ), and non-neurons (mainly glial cells) ${ }^{305}$. However, neurons and glial cells (especially astrocytes) were two major cell types. Without the specific statement, the cell refers to the cortical cell.

\subsection{Introduction}

Because the regeneration capability of adult nervous system is often limited and existing treatments are restricted by many factors, like immunological rejections, potential disease transfer, and inhibitory environment formed after injuries ${ }^{6-8}$, one alternative approach widely studied recently in treatment of injuries in central nervous system (CNS) and peripheral nervous system (PNS) is to fabricate 3D polymeric scaffolds with cells in order to generate tissues suitable for implantation ${ }^{9,10}$.

For neural tissues, multiple cues, such as chemical cues, geometrical cues, and electrical cues, are always presented simultaneously; thus, a platform that could combine different stimuli or cues is highly desired. Nonetheless, most of the previous studies focused only on separating effects of geometrical cues or electrical stimulations. For example, geometrical cues provided by nanofibers have been studied for neuron growth, which proved that nanofibers could mimic the extracellular matrix $(\mathrm{ECM})$ well in terms of their unique characteristics, including ultrafine continuous fibers, high surface-to-volume ratio, high porosity and so on ${ }^{306-309}$. In addition, geometrical cues have also been applied to guide stem cells to differentiated into neural lineage ${ }^{310,311}$, direct orientation of neurons or 
glials ${ }^{159,288,303,310,312,313}$, influence proliferation of stem cells ${ }^{314}$, enhance the neurite outgrowth $^{315-317}$ and guide migration of glial cells ${ }^{108,159}$. Recently, conductive nanofibers are emerging as one of the most important tools in neural tissue engineering due to the geometrical cues they provide for cell growth and functional expression, as well as the possibility of inducing external electrical stimulations for regulation of cellular behaviors $^{83,198,318,319}$. Besides, many studies showed enhanced neurite growth and neuron development caused by electrical stimulations and geometrical cues in 2D conductive nanofibers ${ }^{10,20,288,312,320}$, which prevent cells from infiltration and growth in three-dimension. As a result, studies of electrical stimulation on cortical cells cultured in 3D scaffolds have not been reported previously.

Polyacrylonitrile (PAN) is a safe material for biomedical use such as drug delivery, wound dressing, implant materials, and dialysis membrane ${ }^{278,279}$. Its nanofibers, having diameters in the range from $100 \mathrm{~nm}$ to a few microns, could be easily produced via the electrospinning technology ${ }^{280,281}$. These nanofibers were widely used in tissue engineering as scaffolds ${ }^{282-284}$. Furthermore, the superior mechanical properties of its fibers ${ }^{279}$ make it potential to build a stable 3D scaffold under mechanical loading. Considering the Core-Sheath structure of nanofiber could result to better mechanical properties ${ }^{285}$, we coated a layer of PPy to improve mechanical properties of PAN nanofibers. The PPy was chosen as the coating layer of the nanofibers because of its widespread use in numerous applications in tissue engineering owing to its biocompatibility, easy manipulation, good stability, and high electrical conductivity ${ }^{150,286-288}$. Although the polypyrrole is considered non-biodegradable and might remain in tissue for a relatively long period, its 
biocompatibility in vitro and in vivo has been demonstrated. Within a long period of half year, light inflammation could be found after a PPy-coated silicone tube was implanted to bridge the gap of rat sciatic nerve ${ }^{287}$. However, the acceptable biocompatibility, easy preparation, and good conductivity make it as a commonly used material in tissue engineering studies.

In this report, a 3D conductive nanofibrous scaffold (3D-CNFs), which was made of PPy coated electrospun polyacrylonitrile (PAN) nanofibers, was utilized to achieve 3D cortical cell cultures in vitro. Due to its ease of synthesis, good biocompatibility, and conductivity, PPy was coated to introduce conductivity into the PAN nanofibers while the 3D porous structures were maintained ${ }^{10,274,288}$. Observations confirmed that cells could grow inside the fluffy 3D-CNFs, which was then compared to cells cultured on 2D nanofiber meshes. The combined effects of the 3D nanofibrous structure and the PPy coating on cortical cell growth were observed after one-week culture and evaluated in terms of neuron morphology compared to that in 2D-CNFs and bare PAN nanofibers in both $3 \mathrm{D}$ and $2 \mathrm{D}$. Furthermore, we reported the influence of electrical stimulation on the proliferation of cortical cells and the maturation rate of neurons cultured in these 3D-CNFs, which were also accelerated by the electrical stimulation through 3D-CNFs.

\subsection{Materials and Method}

\subsubsection{Fabrication of PAN nanofibers and Conductive Nanofibers}

The fabrication process of scaffolds followed previous work and illustrated in Figure 
5-1(a). Briefly, the polyacrylonitrile (PAN) $(\mathrm{Mn}=90,000)$ was dissolved in $\mathrm{N}$, $\mathrm{N}$-dimethylformamide (DMF) at a concentration of $10 \mathrm{wt} \%$. The prepolymer solution was constantly stirred until it became homogenous. The electrospinning process was conducted with a customized system. The solution was fed into a syringe, which was capped with a needle ( 22 gauge, blunt) and pushed by a syringe pump at a rate of $1.0 \mathrm{ml} / \mathrm{h}$. A continuous jetting stream was generated by applying a voltage of $15 \mathrm{kV}$. The distance between the syringe tip and the collector was $10 \mathrm{~cm}$. 2D PAN nanofibers were deposited onto the aluminum foil-covered collector. For the 3D nanofibers, the PAN nanofibers were spun into the container filled with ethanol solution and the container was shaken every $5 \mathrm{~min}$ to let the nanofibers extend fully for the 3D architecture. The 3D PAN nanofibers were then obtained by freeze drying method after washing in deionization (DI) water. The final porosity was controlled by adjusting the volume of DI water to achieve a $0.125 \mathrm{mg} / \mathrm{ml}$ Nanofiber_Mass/Water_Volume (M/V) as mentioned in Section 4.3.1. The conductive polypyrrole (PPy) shell layer was coated on the surface of the nanofibers by in-situ polymerization of pyrrole within $\mathrm{FeCl}_{3}$ as the oxidant as mentioned in Section 4.3.1.

\subsubsection{Cell Isolation}

Historically, primary cells isolated from embryonic brain have been more commonly utilized than cells isolated from postnatal brain. It is because of the ease of isolating embryonic, increased viability, improved regenerative ability, and reduced glial growth $^{321,322}$. For same reason, commercial cell lines are also popular. However, additional cell types would more reliably recapitulate the biological environment relevant to the adult 
CNS. So, primary cortical cells from Postnatal 1-day rats were used here.

All animal work was approved by the Nanyang Technological University Institutional Animal Care and Use Committee and abided by the Guidelines on the Care and Use of Animals for Scientific Purposes as set out by the National Advisory Committee for Laboratory Animal Research. The animal work was conducted by the author's colleague who has the IACUC certification. Cortical tissue was isolated the cerebral cortex region of the postnatal 1-day old rat pups (P1) as described in the previous work ${ }^{323,324}$. The tissue was dissociated by trituration after digestion with $20 \mathrm{U} / \mathrm{ml}$ papain (Life Technologies, Singapore) in a dissection buffer adjusted to neutral $\mathrm{pH}$ and allowed to digest until a smooth homogeneous precipitate was formed. The cells were then suspended in a medium consisting of $50 \%$ minimal essential medium (MEM) supplemented with $10 \%$ fetal bovine serum and 50\% Neurobasal medium supplemented with B27 and $0.5 \mathrm{mM}$ glutamine, 25 $\mu \mathrm{M}$ glutamate (Life Technologies, Singapore).

\subsubsection{Cell Culture}

The 3D scaffolds and 2D meshes were fitted into 24-well plates and sterilized by soaking in $70 \%$ ethanol solution for 12 hours, followed by washing 3 times with phosphate buffered saline (PBS). The samples were sterilized under UV for 2 hours. Before seeding cells, samples were incubated with the medium at $37{ }^{\circ} \mathrm{C}$ for half an hour. $100 \mu \mathrm{L}$ medium with a concentration of $5 \times 10^{6}$ cell $/ \mathrm{mL}$ were added to the surfaces of samples after medium removal. The cells were the mixtures of neurons and other cells (mainly glial cells) dissociated from the cortices without sorting and passaging. The cell-laden samples were 
incubated at $37{ }^{\circ} \mathrm{C}$ with $5 \% \mathrm{CO}_{2}$ for 30 min to allow cells to attach. After that, $900 \mu \mathrm{L}$ fresh medium was added to the wells. The cell-laden samples were stored in the incubator since then. After 24 hours, the 24 -well plate was shaken gently, and the unattached and dead cells were removed by removing the old medium entirely through pipetting followed by adding the fresh and pre-warmed medium. After that, half medium was changed with pre-warmed fresh medium twice per week.

\subsubsection{Electrical Stimulation}

Electrical stimulation of cell culture was performed on the 3D-CNFs with a self-made bioreactor as showed in Figure 5-1. Isolated cells were seeded into 3D-CNFs at $5 \times 10^{5}$ cells per well. After $24 \mathrm{~h}$ in culture, stainless steel electrodes were inserted to form the electrical contact with the 3D scaffolds. Since the sample has a diameter of $15 \mathrm{~mm}$ and a height of $5 \mathrm{~mm}$, and the distance between two electrodes was $10 \mathrm{~mm}$, the inserted electrodes would press on the samples. A $100 \mathrm{~Hz}$ pulsed electrical field of $100 \mathrm{mV} / \mathrm{cm}$ was applied across the two electrodes for $4 \mathrm{~h}$ in the incubator (at $37{ }^{\circ} \mathrm{C}$ with $5 \% \mathrm{CO}_{2}$ ) with a function generator (AFG3022C, Tektronix, USA) daily for one week. Cells were analyzed $24 \mathrm{~h}$ after electrical stimulation.

\subsubsection{Immunocytochemistry and SEM Imaging}

To assess the cell culture after 7 days, different antibodies were utilized. Anti-Microtubule-Associated Protein-2 (MAP2) (Millipore, Singapore) and anti-Tau (Abcam, Singapore) were chosen to stain the neuron body and axon respectively. Glial 
fibrillary acidic protein (GFAP) (anti-GFAP antibody, Millipore, Singapore), which is expressed by numerous cell types of the CNS including astrocytes and ependymal cells $^{325,326}$, were utilized to stain glial cells.

Cell-laden samples were rinsed with $1 \times$ PBS and fixed in $10 \%$ neutral buffered formalin solution (Sigma, Singapore) for half an hour. After rinsing with PBS for three times, cells were then treated with $0.5 \%$ Triton X-100 solution (Sigma, Singapore) for $15 \mathrm{~min}$ at room temperature and followed with 3 times PBS rinsing. Cells were then incubated in blocking buffer solution consisting 3\% bovine serum albumin (BSA) (Sigma, Singapore) and 0.5\% fetal bovine serum (FBS) (Life Technologies, Singapore) for $45 \mathrm{~min}$ to avoid non-specific binding of antibodies. After removing the blocking buffer solution, the cells were incubated with neuron-specific anti-Microtubule-Associated Protein-2 (MAP2) (Millipore, Singapore) and anti-Tau (Abcam, Singapore) antibodies in 1:500 dilution at $4{ }^{\circ} \mathrm{C}$ for overnight. On the following day, the samples were rinsed with PBS for 3 times and the cells were then exposed to the Alexa Fluor ${ }^{\circledR} 488$ and Alexa Fluor ${ }^{\circledR} 555$ (Life Technologies, Singapore) in 1:700 dilution at $4{ }^{\circ} \mathrm{C}$ for overnight. Finally, samples were rinsed with PBS again and cell nuclei were stained with 1:1000 diluted DAPI (Life Technologies, Singapore) for $1 \mathrm{~min}$ at room temperature. The final samples were rinsed with PBS and stored in PBS at $4{ }^{\circ} \mathrm{C}$ for further imaging.

Synaptophysin antibody (Life Technological, Singapore), as a pre-synaptic biomarker, and Post-synaptic density protein 95 (PSD95) (Millipore, Singapore), as a post-synaptic biomarker, were utilized to characterize the synapse. Cells fixed and treated with Triton X-100 as mentioned before were incubated with Synaptophysin and PSD95 in 1:1000 
dilution at $4{ }^{\circ} \mathrm{C}$ for overnight. On the following day, rinsed cells were exposed to the Alexa Fluor ${ }^{\circledR} 488$ and Alexa Fluor ${ }^{\circledR} 555$ (Life Technologies, Singapore) in 1:700 dilution at room temperature for one hour. Finally, cell nuclei were rinsed with PBS and stained with 1:1000 diluted DAPI (Life Technologies, Singapore) for $1 \mathrm{~min}$ at room temperature. The final samples were rinsed with PBS and stored in PBS at $4{ }^{\circ} \mathrm{C}$ for further imaging. Fluorescent images from the stained samples were acquired using an inverted confocal laser scanning microscope (Zeiss LSM780). Scaffolds with fixed cells were then washed with DI water and lyophilized for SEM imaging.

\subsubsection{Proliferation and Maturation}

The proliferation of all cortical cells was characterized by PrestoBlue cell viability kit (Life Technologies, Singapore). Briefly, the 3D-CNFs with cell culture were washed and incubated in medium containing $10 \%$ PrestoBlue reagent for $1 \mathrm{~h}$ in a humidified atmosphere at $37{ }^{\circ} \mathrm{C}$ and $5 \% \mathrm{CO}_{2}$. Culture medium containing $10 \%$ PrestoBlue reagent was incubated with no cells and served as the blank control. The absorbance of the reduced PrestoBlue reagent was measured at $570 \mathrm{~nm}$ and $600 \mathrm{~nm}$ as the reference with a Multiskan Spectrum microplate reader (Thermo Scientific, Singapore). The absorbance reading was utilized to represent the cell amount as the manufacturer's protocol.

The maturation was described as the ratio of the number of mature neurons to the total number of neurons. The confocal images were analyzed by Imaris (Bitplane, USA) to quantify cell amount based on fluorescent images. Cells with MAP2 expression (green fluorescence) were counted as neurons. Among them, the cells with tau expression (red 
fluorescence) were counted as mature neurons. Although MAP2 and tau are all mature neuron markers, MAP2 and tau occur in different stages of neuron development, with MAP2 being required for initial process formation and tau being essential for the development of polarity. ${ }^{327}$. Thus, tau expression was used here to assess the degree of cell maturation as a previous study ${ }^{198}$. The cell number was counted with Imaris (Bitplane, USA) by analyzing the fluorescent images. “

\subsubsection{Statistical Analysis}

Statistical analysis of proliferation and maturation was performed using two-way Analysis of Variables (ANOVA) followed by Tukey's post-hoc tests. When $\mathrm{p}<0.05$, results were considered as significant and indicated with *. All experiments were done in triplicate unless otherwise stated. Data in this part were presented in term of mean \pm standard deviation $(n=3)$. 


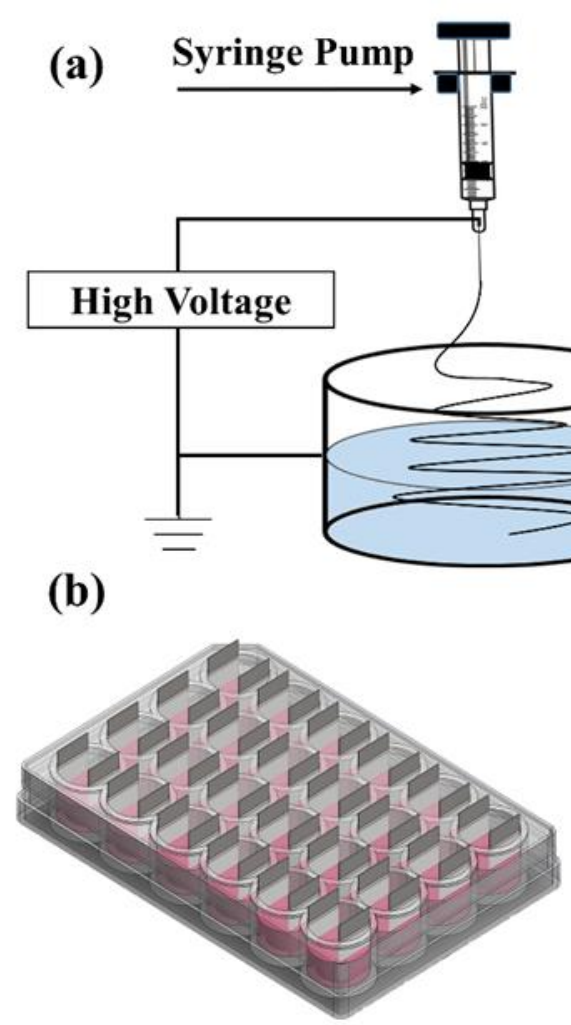

3D View

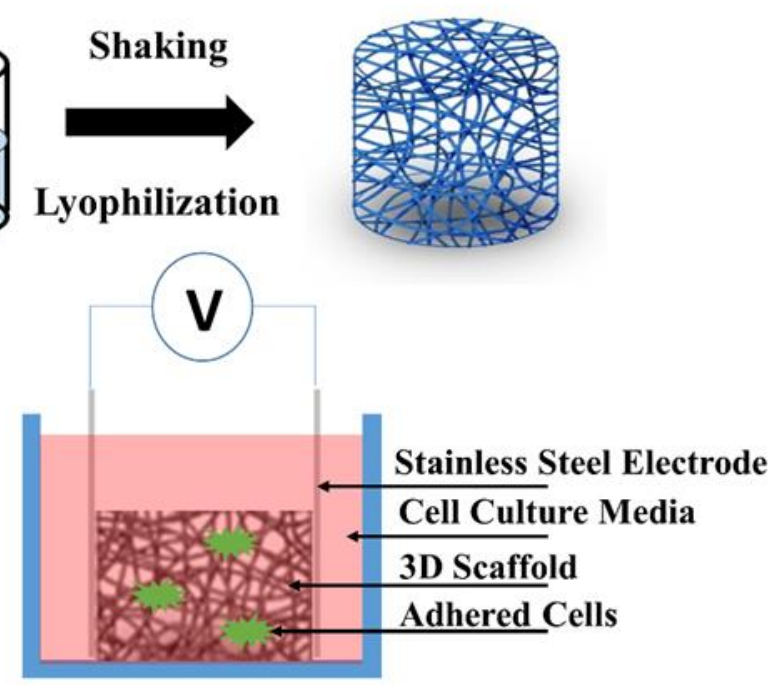

Cross-Section

Figure 5-1 Schematic of (a) electrospinning setup and (b) a bioreactor for electrical stimulation (3D View and Cross-section).

\subsection{Results}

\subsubsection{Characterization of CNFs}

The photograph fabricated NFs and CNFs were showed in Figure 5-2 as well as the SEM images of 2D- and 3D- CNFs. In the photograph, a distinct difference between samples could be observed. The 2D samples were like a thin film (Figure 5-2 (a) (b)) while the 3D samples had clear 3D architectures (Figure 5-2 (d) (e)). While the nanofibers in 2D-CNFs exhibited a close-packed structure (Figure 5-2 (c)), the 3D-CNFs scaffold had an obvious 3D nanofibrous architecture (Figure 5-2 (f)), and nanofibers dispersed in a 3D space 
without collapsing onto a single layer, which produced enough spaces to allow cells to penetrate inside the 3D scaffold. However, the 2D-CNFs could only allow cells to spread on the flat surface. As a result, the 3D-CNFs could provide 3D support to cells inside the 3D scaffold while 2D-CNFs could only function as a 2D support. This ECM-like 3D support is known to affect cellular response and functions ${ }^{112}$, which would be further discussed in the following sections. The porosity of nanofibers had been shown in Chapter 4, Section 4.4.1.

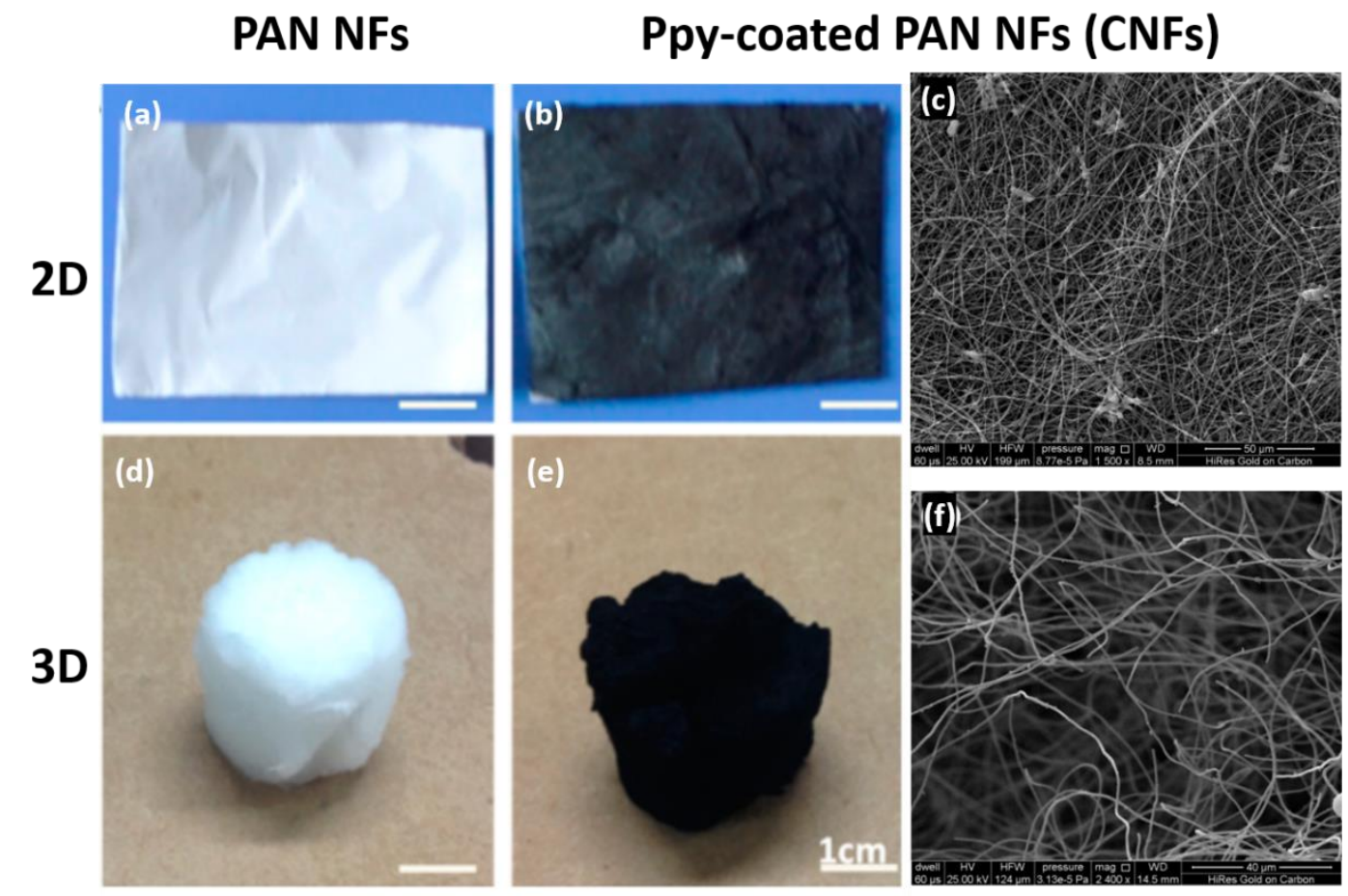

Figure 5-2 Photographs and SEM images of 2D-NFs mat ((a)), 2D-CNFs ((b) (c)), 3D-NFs (d), and 3D-CNFs scaffold ((e) (f)).

The electrochemical performance of the 3D-CNFs was characterized using cyclic voltammetry $(\mathrm{CV})$ as it was widely used in previous studies ${ }^{289,328,329}$. The cyclic voltammetry was performed in the $0.5 \mathrm{M} \mathrm{Na}_{2} \mathrm{SO}_{4}$ solution with a three-electrode system, i.e., the $3 \mathrm{D}-\mathrm{CNF}$ as the working electrode, a platinum wire as a counter electrode, and 
$\mathrm{Ag} / \mathrm{AgCl}$ as the reference electrode. The 3D-CNFs $(20.6 \mathrm{mg})$ were immersed in the $\mathrm{Na}_{2} \mathrm{SO}_{4}$ solution. The voltammograms were recorded with a scan rate of $50 \mathrm{mV} / \mathrm{s}$. The $\mathrm{CV}$ spectrum shown in Figure 5-3 indicated the 3D-CNFs exhibited high charge carrying capacity for a given voltage and excellent electrochemical property. It is most likely attributed to the large surface of 3D-CNFs, which promotes both the incorporation of dopant ions into the matrix and formation of packed PPy chains, thus permitting efficient electron transfer between the polymer chains.

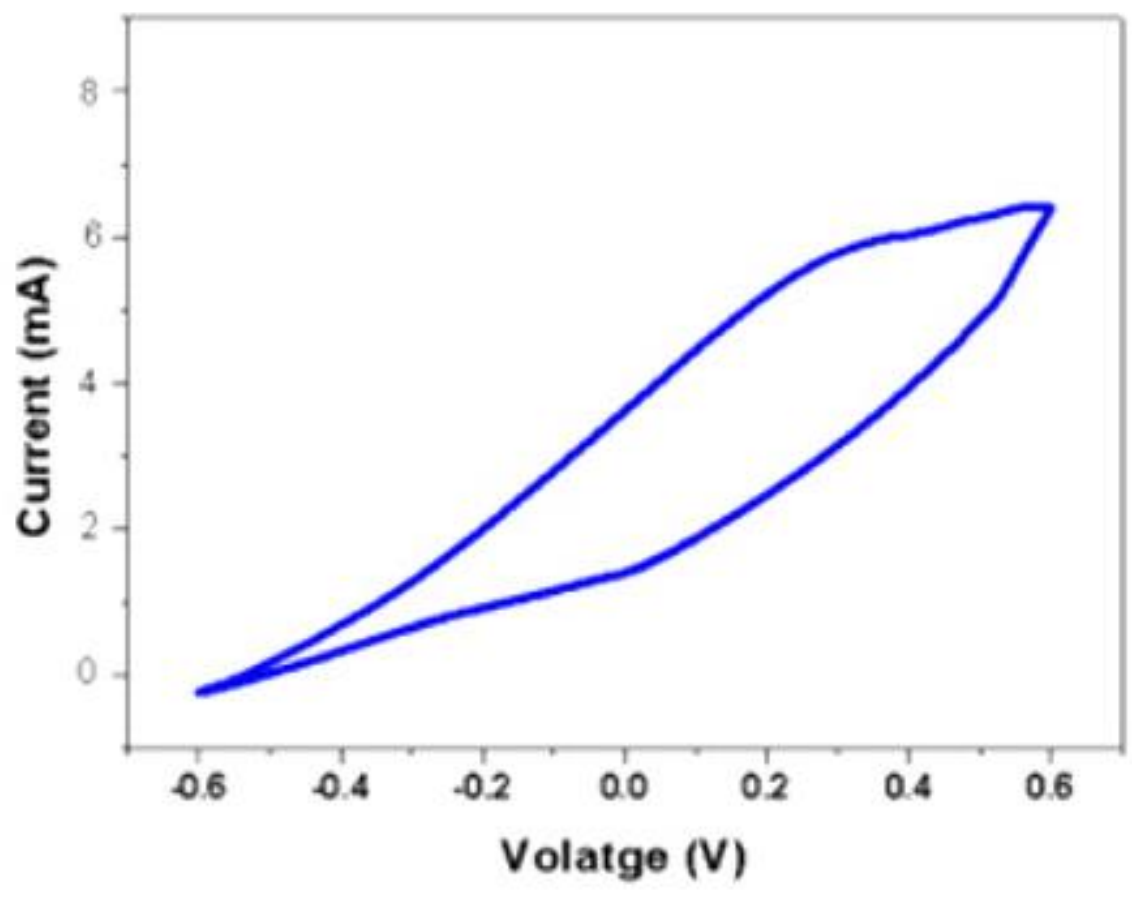

Figure 5-3 CV curves of 3D-CNFs at s scan rate of $50 \mathrm{mv} / \mathrm{s}$.

\subsubsection{Cell Culture in CNFs and PAN nanofibers}

Cortical cells here were utilized to describe the mixture of cells obtained from rat cortices.

It contains neurons, glial cells and some other cells ${ }^{330,331}$. However, neurons and glial cells (especially astrocytes) were two major cell types ${ }^{332}$.

To assess cell culture in CNFs and PAN nanofibers, fluorescent images of cells cultured in 
CNFs and PAN nanofibers were shown in Figure 5-4. Firstly, the neuron-specific fluorescent images stained with anti-MAP2 antibody (Green) demonstrated the existence and validity of neurons in the 2D-PAN nanofibers (Figure 5-4(a)), 3D-PAN nanofibers (Figure 5-4(c)), 2D-CNFs (Figure 5-4(b)) and 3D-CNFs (Figure 5-4(d)). In all kinds of nanofibers, cells are able to form extended neurites and connections with each other (observed from the images), which are essential for cell signal transmission ${ }^{333}$. (The connections in 3D-CNFs were characterized in Section 5.4.4) However, we observed most of neurons cultured in both 2D and 3D PAN nanofibers (Figure 5-4 (a) and (c)) tended to grow individually with loose neurite connections. The soma-to-soma contact (soma was represented by nuclei (blue spots), which were inside neurons (MAP2, green)) was hardly seen in these bare PAN nanofibers (Figure 5-4 (a) and (c)) as 7 out of 9 confocal images (not shown) of cells on bare PAN nanofibers showed no clusters. On the other contrary, most of neurons cultured in 2D-(Figure 5-4 (b)) and 3D-CNFs (Figure 5-4 (d)) tended to grow together to form cell clusters as 7 out of 10 confocal images of cells in CNFs showed clusters. In general, low densities and loose connections could be obviously observed in bare PAN nanofibers, while larger cell clusters could be easily discovered in CNFs. This phenomenon could be seen in both $2 \mathrm{D}$ and $3 \mathrm{D}$ samples, which may be attributed to the PPy coating. 


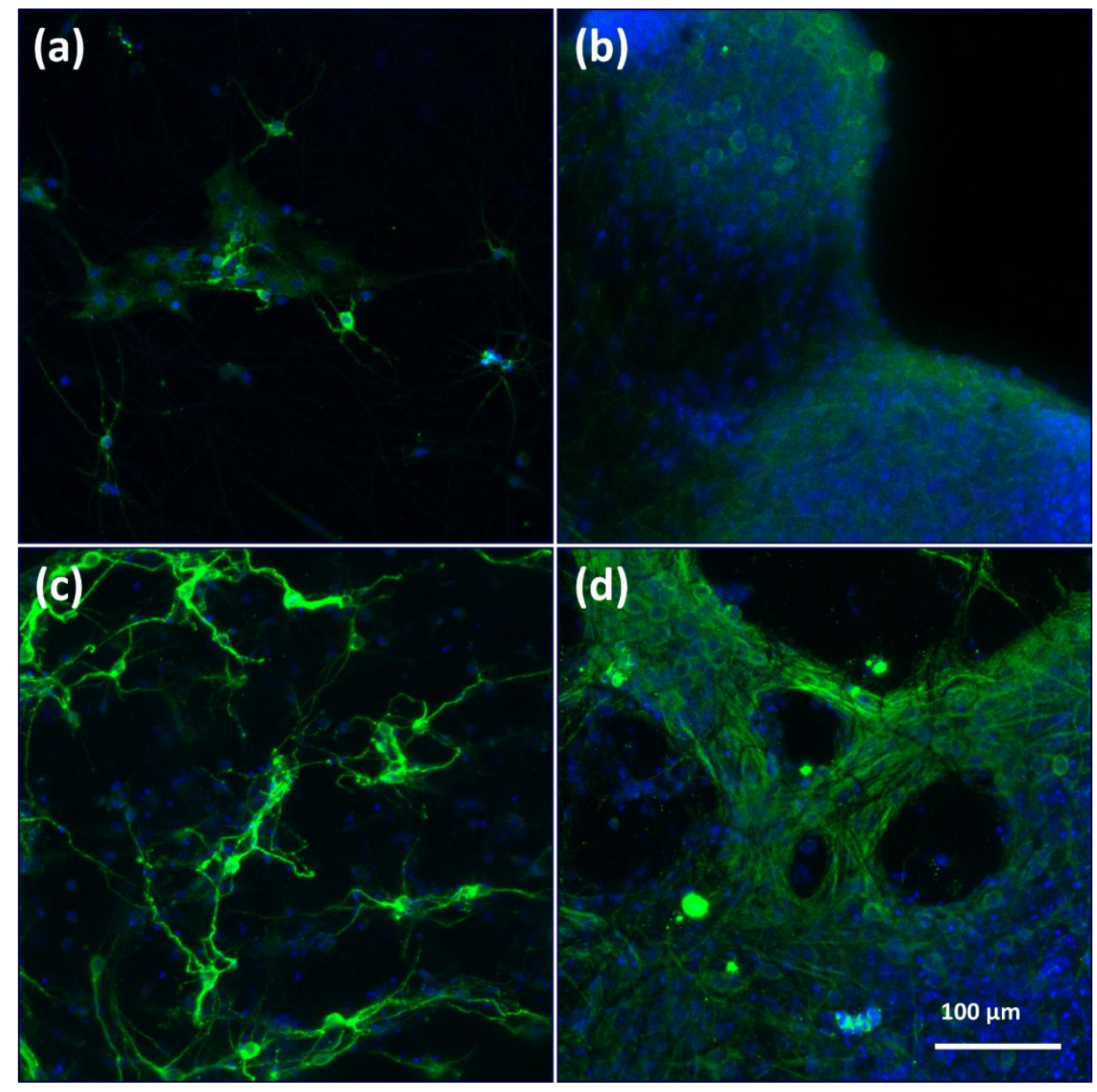

Figure 5-4 Fluorescent images of anti-MAP2-stained neurons in (a) 2D-PANs, (b)

2D-CNF nanofibers, (c) 3D-PAN and (d) 3D-CNF nanofibers. Green: MAP2; Blue: DAPI.

\subsubsection{Cell Culture in the three-dimension}

SEM and confocal images were taken to evaluate the cortical cell morphology in 2D and

3D nanofibers. First, SEM images of cortical cells cultured onto the surface of 2D-CNFs

(Figure 5-5 (a) and (c)) and in the interior of the 3D-CNFs scaffolds (Figure 5-5 (b) and

(d)) after 7-day in vitro culture showed that cortical cells with round morphology

(indicated by red arrows) and flat morphology (indicated by red triangles) successfully 
adhered to both 2D nanofiber meshes and 3D-CNFs, which demonstrated these nanofibers were suitable for cortical cell attachment and survival in vitro. The large and flat cells (indicated by red triangles) were probably glial cells since neurons usually have a round soma. Compared to glial-like cells spread on the mesh surface in 2D-CNFs (Figure 5-5 (a)), cells formed 3D structure inside the 3D-CNFs (Figure 5-5 (b)). As showed in Figure 5-5 (c), cortical cells in 2D-CNFs, formed intimate contacted with multiple fibers due to the dense structure in the vertical direction only allowed them to grow along the $2 \mathrm{D}$ surface instead of filtrating into the scaffolds. In contrast, cortical cells cultured in the interior of 3D scaffolds could adhere along the fibers due to the 3D architecture of scaffolds as indicated in Figure 5-5 (d). Although some cells in Figure 5-5 (c) were under the nanofibers of first layer, a deeper infiltration would be prevented due to the close-packed nanofibers in Z-direction compared to that in 3D-CNFs.

Furthermore, the 3D confocal microscopic ortho-images (Figure 5-5 (e) and (f)) confirmed the 3D neuronal network formation in our 3D-CNFs (Figure 5-5 (f)) and crossed a distance more than $100 \mu \mathrm{m}$ in the Z-direction while the culture in 2D-CNFs (Figure 5-5 (e)) was obviously flat. A larger depth could be expected because only part of the whole neuronal network was captured in the view field due to the limitation of our facility. Hence, we confirmed that the fluffy structure of our 3D-CNFs could help neurons and glial cells filtrate and migrate into the scaffolds thus formed a 3D microenvironment closer to the native microenvironment and could be predicted to enhance the cell-cell and cell-microenvironment interactions. In contrast, the dense structures of 2D-CNFs prevented cells from migrating into the deeper layers. The 3D-CNFs were then selected to 
study the incorporated effect of 3D-CNFs and electrical stimulation on cells.
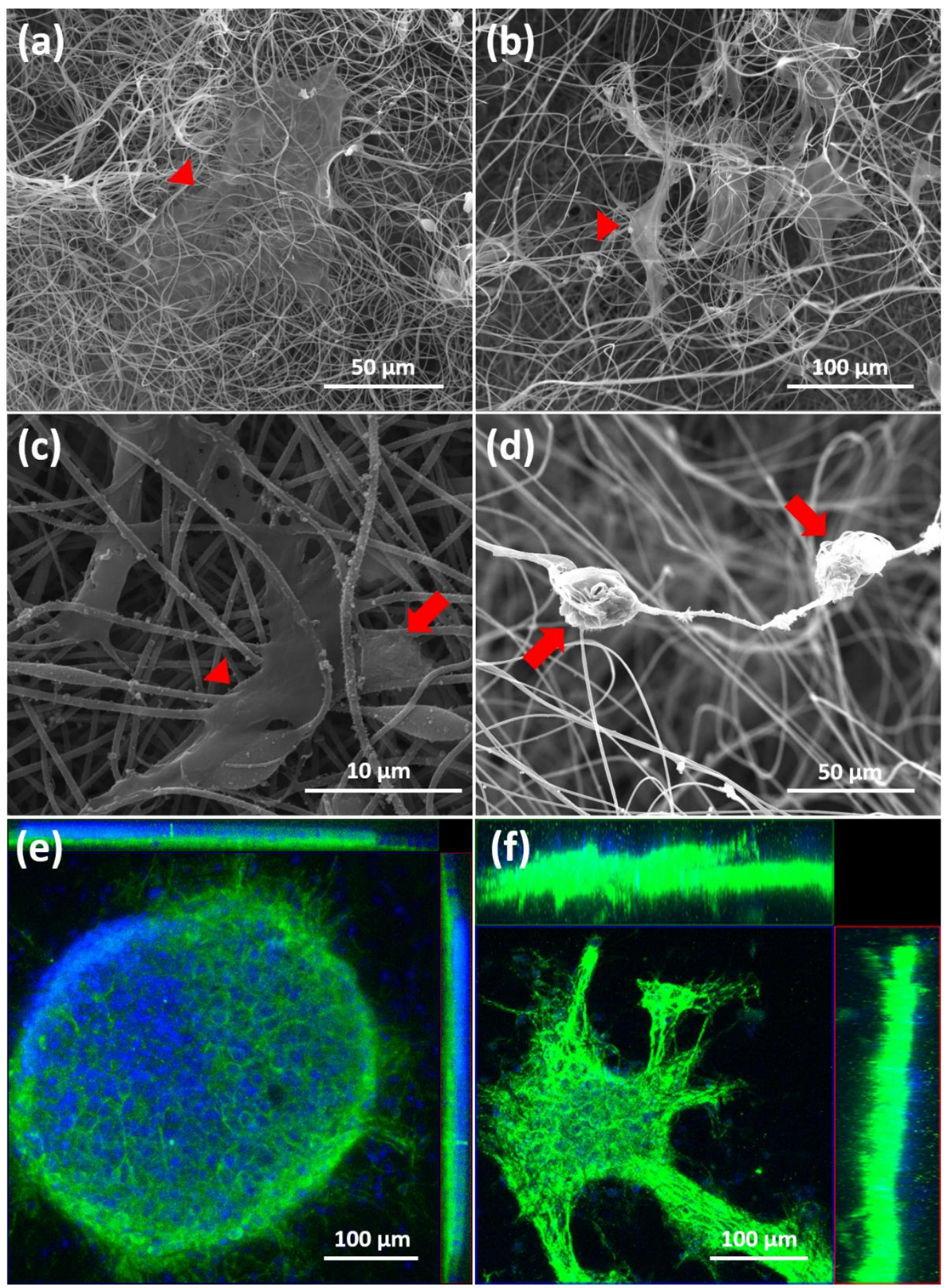

Figure 5-5 SEM of cortical cells cultured in 2D-CNFs (a, c), 3D-CNFs (b, d) and confocal

microscopic ortho-images of cell culture in 2D-CNFs (e) and 3D-CNFs (f). The red arrows and triangles indicated two different cell morphologies respectively. Green: MAP2; Blue:

\section{DAPI.}




\subsubsection{Effect of ES on Cell Morphology}

To study the influence of electrical stimulation on cortical cells (mainly focused on neurons and astrocytes), cortical cells were cultured in 3D-CNFs for 7 days with daily pulsed electrical stimulation (ES). In non-ES cultures (control), most neurons aggregated together to form clusters; and neurites (indicated by red line) were found to connect those isolated clusters (Figure 5-6(a)). Unlike the unstimulated 3D-CNFs cultures ES altered the morphologies of cells, i.e., no isolated clusters found in the electrically stimulated cultures (Figure 5-6(b)). Instead, the electrically stimulated neurons tended to grow individually with neurite to connect individual neurons. The neurite connections were marked with red lines (in Merge) using Simple Neurite Tracer ${ }^{334}$.

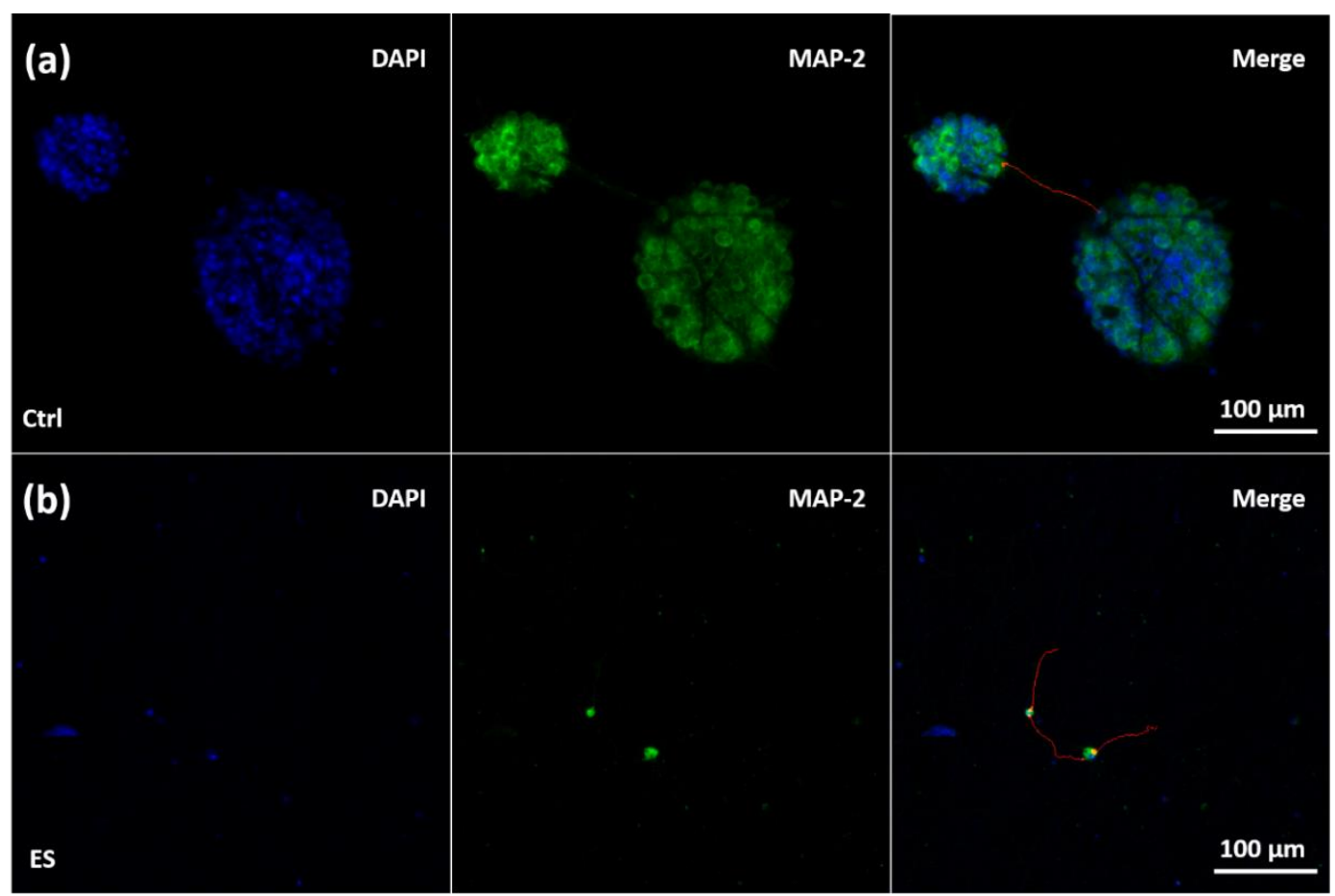

Figure 5-6 Confocal fluorescent images of neuron morphology after 7-day culture in the

3D-CNFs (a) without and (b) with electrical stimulation. Neurite connections between individual neurons and connection between clusters were marked with red lines. 
Both neurons and glial cells (mainly astrocytes) were stained in the Figure 5-7 by anti-MAP2 antibody and anti-GFAP antibody respectively. As mentioned before, neurons cultured without ES (Figure 5-7 (a-d)) aggregated while neurons cultured with ES ((Figure 5-7 (e-h))) grew more independently. The MAP2 expression and GFAP expression indicate most of the neurons were growing closely with glial cells (astrocytes). Some expectations, such as neuron grew without glial cells around (marked with the red circle in Figure 5-7 (h)) and cells expressed neither MAP2 nor GFAP (marked with the white circles in Figure 5-7 (d)(h)), could be observed. (The yellow color (overlap of the colors) did not mean MAP2 and GFAP stained for the same cell. Since the cells were cultured in a 3D structure, the cells in different planes will cause the overlap of different colors.)

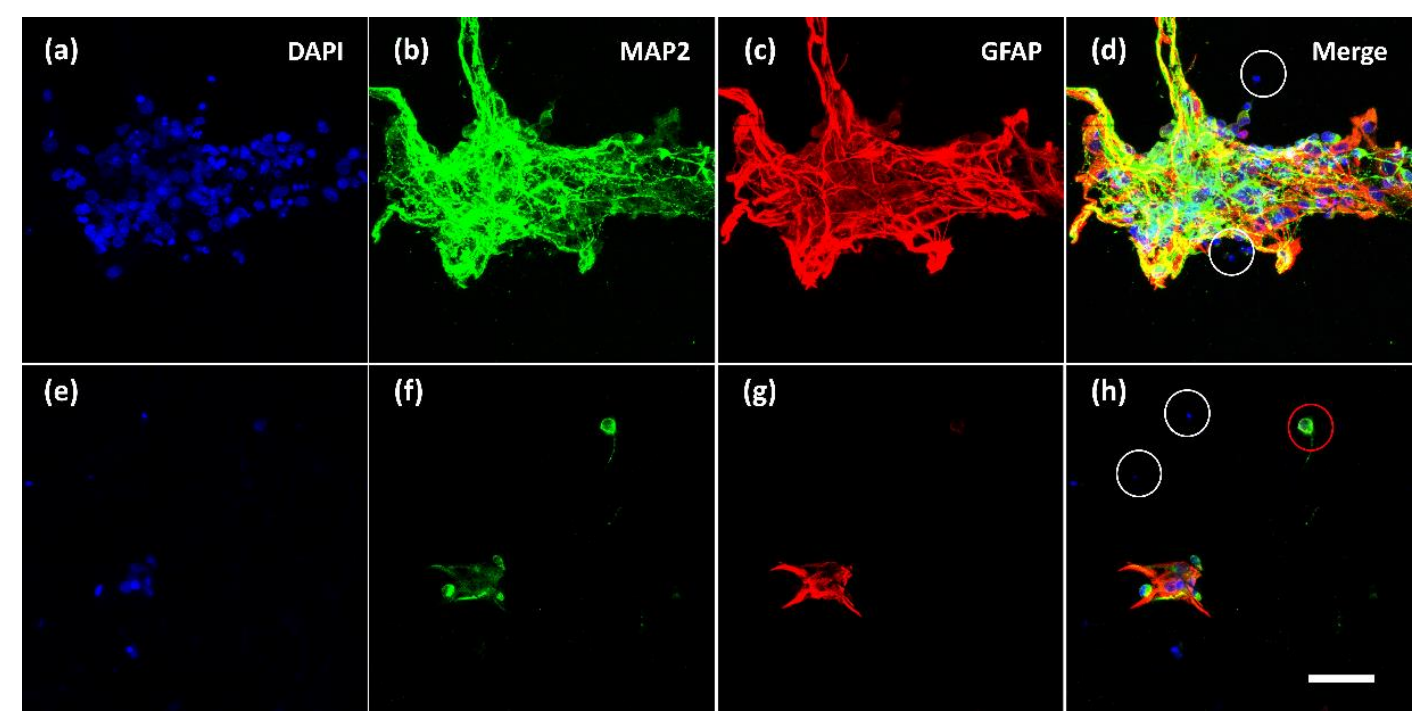

Figure 5-7 Confocal fluorescent images of neurons and glial cells on day 7 in the

3D-CNFs (a-d) without and (e-h) with electrical stimulation. Blue: DAPI; Green: MAP2;

Red: GFAP. Neuron grew without glial cells around was marked with the red circle; cells expressed neither MAP2 nor GFAP marked with the white circles. Scale bar: $100 \mu \mathrm{m}$.

To verify the formation of synapse, immunocytochemistry staining of both synaptophysin 
antibody (Life Technological, Singapore), a pre-synaptic biomarker, and Post-synaptic density protein 95 (PSD95) (Millipore, Singapore), a post-synaptic biomarker, were utilized. The expression of synaptophysin and PSD95 of cells cultured for 7 days with or without ES were shown in Figure 5-8. The cells in both the control group (cell cultured in 3D-CNFs for 7 days without ES) and the ES group expressed synaptophysin and PSD95. The synapses were indicated by the adjacent or overlapped fluorescent spots of synaptophysin and PSD95. 


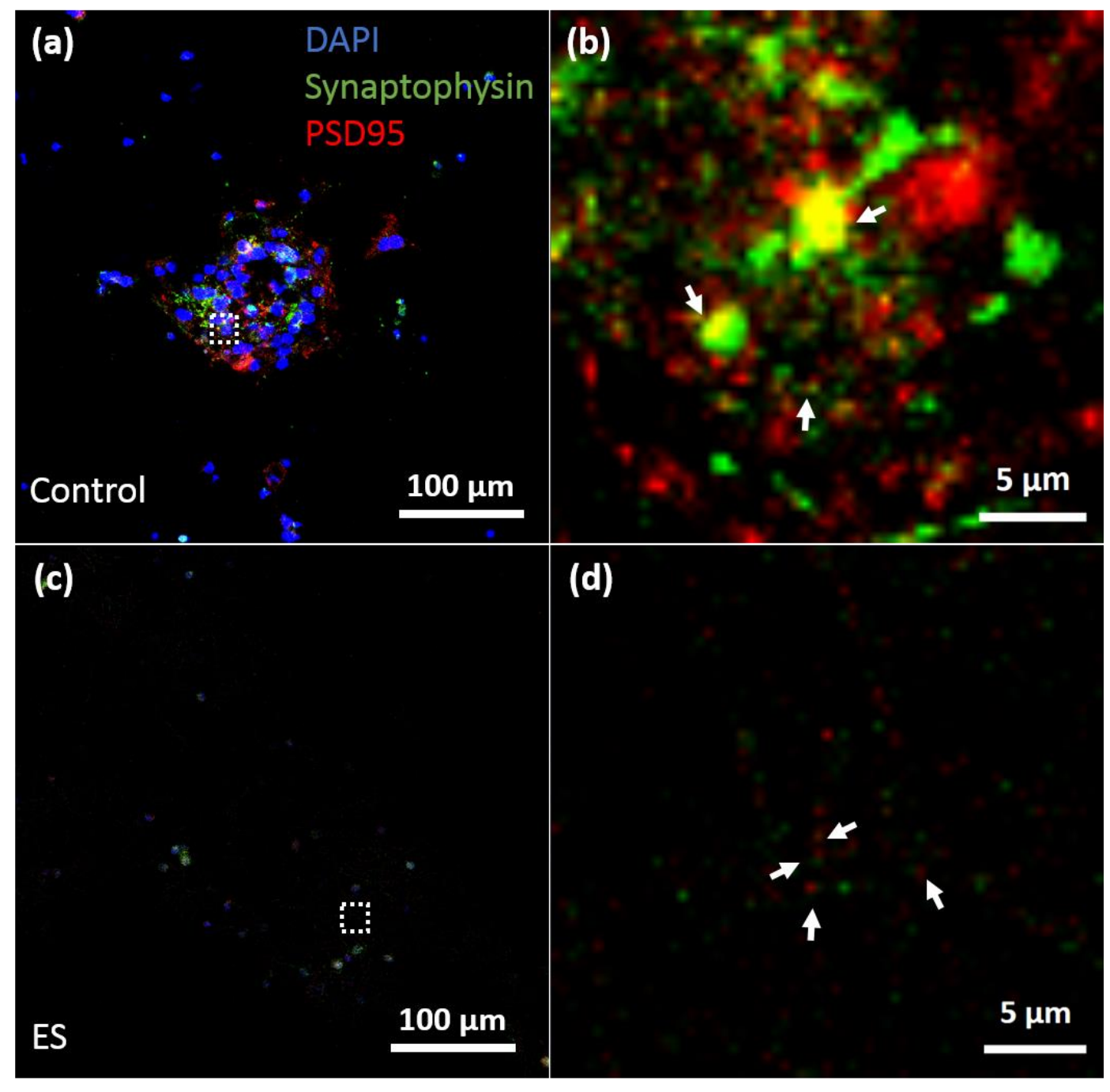

Figure 5-8 Immunostaining of synaptophysin and PSD 95 in 7-day cultured cortical cells

in Control (a)(b) and ES (c)(d) groups. (b) and (d) were regions of interest (indicated with dashed line) from (a) and (c) respectively. The synaptophysin was detected adjacent to PSD 95 (indicated with arrows).

\subsubsection{Effect of ES on Proliferation of Cortical Cells}

As the other major part of cells isolated from cortices besides neurons, glial cells play varied important roles in the entire nervous system, including providing physical support for neurons and regulating the internal environment ${ }^{335}$. To investigate the effect of ES to 
the glial cells, their proliferation in the 3D-CNFs was characterized because mature neurons do not proliferate ${ }^{336}$. Results obtained from PrestoBlue kit were illustrated (Figure 5-9) and the cell amounts were represented by relative absorbance. From day 1 to day 3 , the increase of cell amount was not statistically significant. Although the means of cell amounts in ES groups were always larger than that in control groups, they did not have a statistical difference on day 1 and day 3. However, the proliferation rate increased quickly after day 3 so that the cell amounts in day 7 showed a significant increase compared to day 1 and day 3 in both ES and Control groups. Furthermore, after 7-day culture, the cell amount in 3D-CNFs with ES was statistically larger than that without ES, which indicated a significant improvement on glial cell proliferation caused by ES through the 3D-CNFs. Two-way Analysis of Variance (ANOVA) test gave the main effect value for ES, F $(2,12)$ $=39.92, p<0.0001$ and the main effect value for culture time, $\mathrm{F}(2,12)=18.28, p=$ 0.0011, which proofed that electrical stimulation had even more significant influence than culture time on the proliferation of cortical cells in 3D-CNFs, i.e., cortical cells proliferated with increasing culture time while ES improved the proliferation even more significantly. 


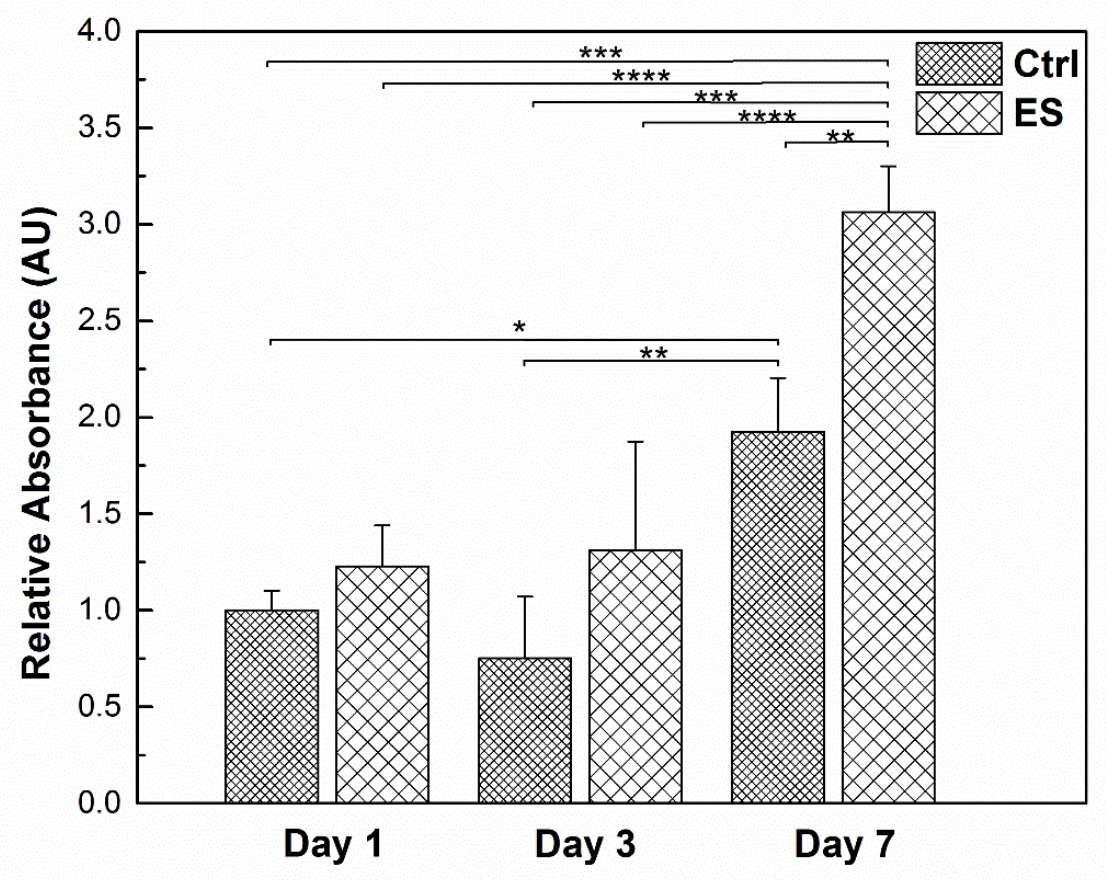

Figure 5-9 Proliferation (represented by relative absorbance in $570 \mathrm{~nm}$ ) of cell cultures with or without electrical stimulation in day 1 , day 3 and day $7 . *$ indicated $p<0.05, * *$ indicated $p<0.01, * * *$ indicated $p<0.001$ and $* * * *$ indicated $p<0.0001$.

\subsubsection{Effect of ES on Maturation of Neurons}

The effect of ES on cell cultures in 3D-CNFs was also characterized by neuron maturation, i.e., the ratio of the number of mature neurons to the total number of neurons (Figure 5-10). Anti-tau antibody staining was utilized to define maturation of neurons ${ }^{198}$, i.e., mature neurons were the neurons with tau expression. The total number of neurons was obtained by counting the cells with MAP2 expression in Imaris (Bitplane, USA) as described in Section 5.3.6. The single-channel and merged fluorescent images indicated the existing of mature neurons after 7-day culture with different morphologies in the control and ES 
group respectively (Figure 5-10 (a) and (b)). Without ES, the maturation in control groups reached $35.70 \%$ on day 1 , then slowly increased to $44.98 \%$ on day 3 and finally reached $46.68 \%$ on day 7 . With ES via $3 \mathrm{D}-\mathrm{CNFs}, 61.07 \%$ of neurons were mature on day 1 followed by a significant jump to $76.75 \%$ on day 3 , which increased stably to $84.27 \%$ on day 7. In addition, a significant enhancement of maturation happened in ES groups compared to control groups could be observed at every time point starting from day 1 to day 7. From a two-way ANOVA, the main effect values were $\mathrm{ES}(\mathrm{F}(2,12)=208.9, p$ $<0.0001)$ and culture time $(\mathrm{F}(2,12)=21.84, p=0.0001)$, which suggested that both ES and culture time contributed significantly on the maturation of neurons in 3D-CNFs (Figure 5-10 (c)). 


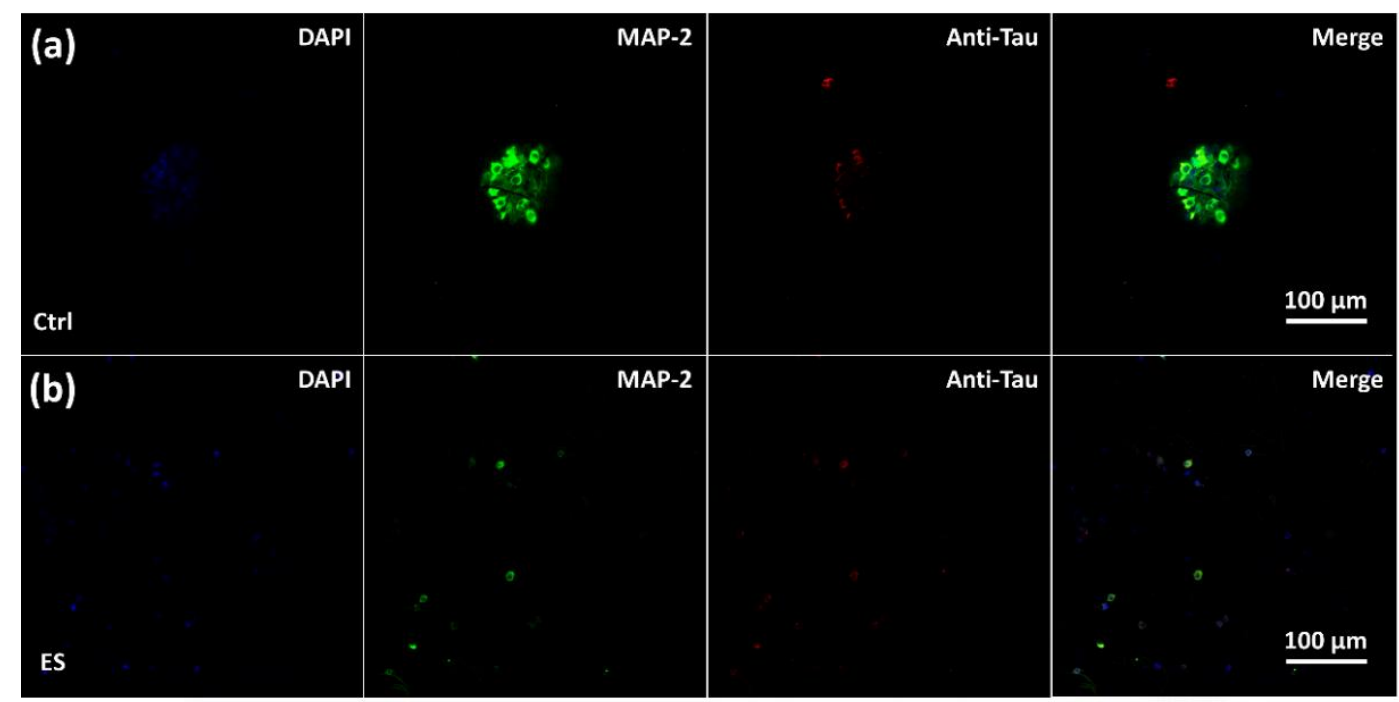

(c)

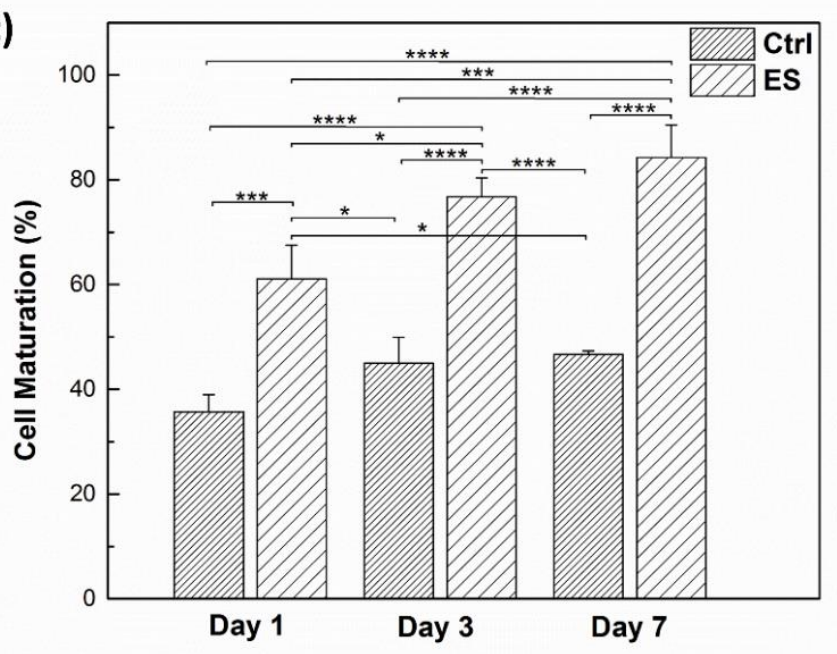

Figure 5-10 Maturation of neurons with or without electrical stimulation in day 1, day 3 and day 7. * indicated $p<0.05, * *$ indicated $p<0.01, * * *$ indicated $p<0.001$ and $* * * *$ indicated $p<0.0001$.

\subsection{Discussion}

Cortical cells cultured in all the 2D and 3D nanofibers grew well and neurons showed a good spreading in these samples. Different from the neurons in PAN nanofibers, the neurons cultured in CNFs (both 2D and 3D) tended to grow together and formed large and dense cellular clusters, which could be caused by the PPy coating. Similar results in 2D 
substrates had been reported that the PPy coated substrate could improve the formation of cell clusters and lead to a higher neuron density compared to uncoated substrate ${ }^{337}$. One possible reason of cluster increase was attributed to the roughness increase in PAN nanofibers after PPy coating because previous studies have found neurons could be negatively affected by surface roughness. In our samples, a rougher surface could be observed in PPy-coated nanofibers as shown in Figure 5-11.

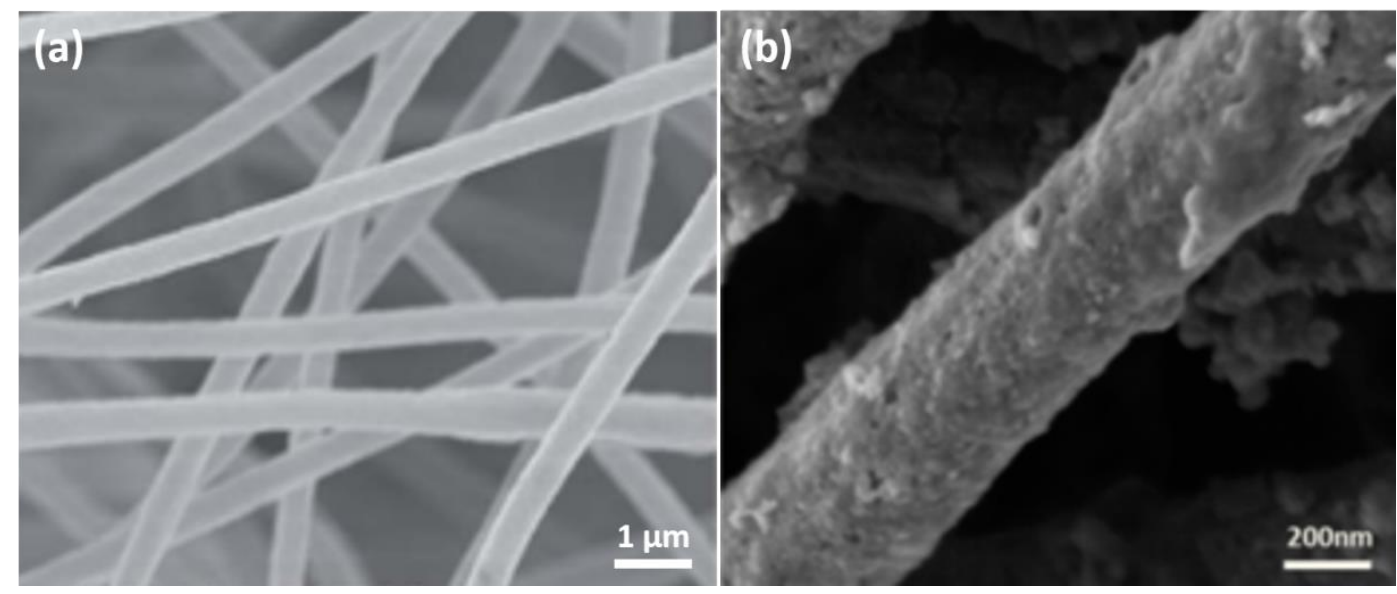

Figure 5-11 Surface roughness of PAN (a) and PPy-coated PAN nanofibers (b). *(a) was reproduced from Ref. ${ }^{338}$ with permission of RSC Advances.

SEM and confocal images clearly showed that the 3D-CNFs as a scaffold performed well to help neurons migrate into the interior space as shown in Figure 5-5 (d) (f) due to their fluffy structure with large interconnected pores, while the 2D-CNFs performed as a 2D substrate and there was no interior space allowing cells to migrate into the interior. Particularly, hundreds of micrometers penetration of cells into the scaffold was observed in the fluorescent image, which further confirmed that the 3D-CNFs could support 3D cell culture. Because the features of cells cultured in $3 \mathrm{D}$ models are closer to the complex in vivo condition, they were induced to behave in a manner that is closer to their behaviors in 
vivo. For example, compared to $2 \mathrm{D}$ culture, the signaling and gene expression of cells cultured in $3 \mathrm{D}$ were more close to those of cells in vivo, and these advantages could help us build a more realistic platform for tissue engineering ${ }^{339}$.

Furthermore, ES changed the clustering behavior of cells cultured in the 3D-CNFs. Despite the fact that the mechanism is not completely clear, it had been reported that the clustering could depend on the surface properties of the scaffolds, such as hydrophilicity $^{340,341}$, geometrical cues ${ }^{342}$, as well as the electrical stimulation ${ }^{19,343}$. In 2D substrates, it was reported that neuronal culture with electrical stimulation may increase neuron clusters ${ }^{19,343}$, which was attributed to the heterogeneous conductivity of the substrate, i.e., the conductivity in the different locations of the substrate was different. However, we found that electrical stimulation in 3D-CNFs prevented the cells from clustering, which could be attributed to the combined effects of the 3D structure and ES. In general, we believe that the combined effects of the 3D nanofibrous scaffold and ES include three parts, i.e., (1) Glial cells could facilitate the migration and spread of neurons $^{341}$. With electrical stimulation, development of both neurons and glial cells could be promoted ${ }^{19,344}$. In our study, the glial cells proliferated and spread across the scaffold as researchers have found the ES regulated astrocytes' proliferation and migration ${ }^{344}$. As shown in Figure 5-7, the glial cells, as the same as the neurons, aggregate in unstimulated 3D-CNFs. However, the mechanism of electrical influence on morphology of cortical cells was unclear. We hypothesize that different from $2 \mathrm{D}$ substrates, the $3 \mathrm{D}$ structure provided a larger space for glial cells spread and migration in three-dimension (Figure 5-5 (b)), thus the larger glial distribution probably allows neurons to spread and to migrate more broadly 
instead of forming clusters. (2) The surface properties of PPy coated nanofiber could be changed during electrical stimulation. For example, it had been reported that an increase of fibronectin absorbance in PPy with electrical stimulation ${ }^{345}$. With the increase of certain protein absorbance in PPy surface, the surface would become more biocompatible and help cells spread, migrate and proliferate, for example, some astrocyte-secreted matricellular proteins are involved in cell proliferation, maturation, migration and so on ${ }^{346}$. It is another possible reason why the electrical stimulation promoted proliferation of glial cells (Figure 5-9). (3) Electrical stimulation could alter local electrical fields of ECM molecules. The perturbations in ECM electric fields via electrical stimulation can alter the conformation and orientation of cytoplasmic biopolymers, such as RNA, DNA and pro-collagen, by changing enzyme-driven reactions through electro-conformational coupling mechanisms as well as affecting downstream signaling within cells $\mathrm{s}^{347}$.

ES has been found to improve the proliferation of glial cells in the 3D-CNFs, which agree with previous results obtained on 2D substrates. Varied studies had been conducted to reveal the relation between electrical stimulation and cell proliferation ${ }^{42,304,344,348-350}$. The proliferation is probably attributed to the elevated calcium level because the voltage-gated calcium channels in the cell membrane could be activated by electrical stimulation ${ }^{42}$. However, further evidence is needed to explain our observation with this existing mechanism. Although the number of neurons is generally assumed to be a key determinant of brain function, glial cells have been valued as a major part of the brain ${ }^{335}$. Regulation of glial cell proliferation could be an important tool in study and regeneration of specific part of the brain as the glia/neuron ratio varies along the brain ${ }^{351}$. 
It is found that ES could accelerate and improve maturation of neurons. With the similar reason mentioned above, the ES-induced accelerated neuron maturation, represented by the ratio of tau expression (Figure 5-10), may also be attributed to the signaling change as well. Particularly, the influx of $\mathrm{Ca}^{2+}$ induced by the depolarizing current, can active the calmodulin-kinases to elicit neurite outgrowth and expedite neurites development ${ }^{20,198}$.

\subsection{Conclusions}

The biocompatibility of PPy-coated PAN nanofibers had been demonstrated by culturing cortical neurons. Morphology with large neuron clusters was observed in PPy-coated nanofibers (CNFs), while neurons in bare PAN nanofibers grew separately. More importantly, the combined effect of the 3D conductive nanofibers and electrical stimulation on neurons and other cortical cells was investigated here. This study enriched the growing body of evidence for using electrical stimulation and conductive nanofibers to influence the culture of cortical cells. The fluffy 3D structure and conductivity exhibited by $3 \mathrm{D}-\mathrm{CNF}$ s provided a novel platform to explore a series of applications in neural tissue engineering. The fluffy $3 \mathrm{D}$ structure was demonstrated to be able to help cells penetrated into the interior space to form 3D cultures. During this study, the pulsed electrical stimulation applied through the PPy coated 3D-CNFs changed the morphology of cell cultures from clustering to dispersion. And electrical stimulation improved the proliferation of cells compared to unstimulated cells in a period of 7 days. Furthermore, neuron maturation in the 3D-CNFs was accelerated by electrical stimulation in 7 days. 


\section{Chapter 6. General Conclusions and Future Work}

\subsection{Conclusions}

From the literature review and our previous study, we have realized that the physical stimuli from the microenvironment could be very important to control cell behavior for potential clinical applications. However, the studies of the effects of physical stimuli applied via substrates/scaffolds have not been comprehensive, and many mechanisms remain unclear. Furthermore, the studies of the combined effects of physical stimuli and other factors, like time-dependence, surface topography, and 3D architecture, are scant. Hence, we hypothesized that the combined effects of different physical stimuli applied via substrates/scaffolds and other factors (e.g., culture time, 3D structures of the scaffold) could be more complex and bring us new perspectives to understand cell-microenvironment interactions.

The mechanical properties of hMSCs (i.e., elastic moduli and viscoelastic properties) were used to study the effects of substrate stiffness and culture time. Change of cell mechanical properties is of great interest due to its relationship with various cellular behaviors, including proliferation, adhesion, and differentiation ${ }^{215}$. However, few studies have been conducted on the influence of culture time, and this work highlighted the influence of both stiffness of substrates and culture time on the hMSCs' mechanical properties. It revealed a time-dependent change of cell mechanical properties responding to substrate stiffness, which could enrich our understanding of cell-microenvironment interactions. 
Although some studies have been conducted to reveal the effect of mechanical loading on cell culture ${ }^{64,116,352,353}$, there are few based on $3 \mathrm{D}$ culture, which could allow cells to migrate and spread in 3D. We developed novel 3D electrospun nanofibers (3D-CNFs) with improved mechanical properties by coating them with a layer of PPy for 3D cell culture. In such a scaffold, the effect of long-term dynamic loading on hMSC was investigated. The combined effect of 3D-CNFs and dynamic loading changed the morphology (i.e., aligned cells along the fibers), and collagen expression of hMSCs was promoted, while the proliferation and osteogenic differentiation were not affected.

With the help of the same 3D-CNFs, cortical cells were cultured in 3D. This study revealed a combined effect of ES and 3D-CNFs on the culture of cortical cells. Pulsed ES applied through the PPy-coated 3D-CNFs changed the morphology of cell cultures from clustering to dispersion in PPy-coated surfaces. ES also improved the proliferation of glial cells as well as neuron maturation compared with unstimulated cells in a period of 7 days. The general objective to use different substrates and scaffolds with specific mechanical cues or electrical stimuli to modulate cell behaviors was achieved. In each chapter, new combined effects of physical stimuli and other factors (i.e., culture time, 3D structure) were investigated. Through our study, we provided some novel findings on cell-microenvironment interactions. However, further studies are required to gain a more comprehensive understanding in future.

\subsection{Future Work}

To further understand the cell-microenvironment interactions, the development of new 
biomaterials which can mimic native cell microenvironment is significant.

* Although stiffness of PDMS showed significant effects on cellular mechanical properties in the long-term culture, there are some aspects could be improved. The elastic moduli of PDMS substrates with the current ratio of base-to-curing agent can only cover the moduli range suitable for muscle and pre-calcified bone. On the other hand, a ratio of base-to-curing agent higher than 30:1 or lower than 5:1 would make curing difficult.

- One future work is to study the effect of stiffness with a material could achieve a wide range of stiffness with stable chemical and physical properties while maintaining the acceptable biocompatibility. Substrates with a wide range of stiffness covering more tissues stiffness could be utilized to study cell response to substrate stiffness systematically.

- Furthermore, the long-term culture requires robust chemical/physical modification for cell adhesion. Although plasma treatment and PLL coating could support cell culture for two weeks without decomposition, covalently bonded surface coating would be more suitable for longer culture period.

- Additionally, the effect of 3D scaffold stiffness on cell behaviors is not well studied, which offers a new direction for future study. The 3D scaffolds could better mimic cells' native environment than 2D substrates, so a study on effects of matrix stiffness on cell behaviors in 3D culture is attractive.

* A novel scaffold was used in this thesis to study the effect of dynamic loading in 3D hMSC culture. However, an alternative material to replace PPy-coated PAN 
nanofibers would be the next breakthrough.

- Both PPy and PAN are not biodegradable which limited their use in future clinical applications. An elastic scaffold with controllable biodegradability could be used to improve the in-vitro development of tissues by applying dynamic loading.

* In the study of the effects of electrical stimulation on 3D culture, the same requirement for a new material comes up.

- The PPy-coated scaffold was limited in future clinical applications because the material is not biodegradable. Conductive materials with good biodegradability or a new method to induce electrical stimulation on existing biodegradable materials would be the important future work.

- Furthermore, the PPy-coated surface itself would cause the neuron clustering which is not desired in many applications of neural engineering. So, the next challenge is to invent a conductive 3D scaffold with better surface properties to support better neuron culture without clustering.

In summary, a scaffold can induce different biophysical cues simultaneously is extremely attractive in the study of cell-microenvironment interactions. Although the 3D-CNFs could induce different cues (e.g., 3D structure, geometrical cues, mechanical loading, electrical stimulation), improvements in biodegradability would be needed. 


\section{References}

(1) Langer, R.; Vacanti, J. Tissue Engineering. Science (80-. ). 1993, 260 (5110), 920926.

(2) Griffith, L. G.; Naughton, G. Tissue Engineering--Current Challenges and Expanding Opportunities. Science 2002, 295 (5557), 1009-1014.

(3) Giordano, A.; Galderisi, U.; Marino, I. R. From the Laboratory Bench to the Patient's Bedside: An Update on Clinical Trials with Mesenchymal Stem Cells. J. Cell. Physiol. 2007, 211 (1), 27-35.

(4) Pittenger, M. F.; Mackay, A. M.; Beck, S. C.; Jaiswal, R. K.; Douglas, R.; Mosca, J. D.; Moorman, M. A.; Simonetti, D. W.; Craig, S.; Marshak, D. R. Multilineage Potential of Adult Human Mesenchymal Stem Cells. Science 1999, 284 (5411), $143-147$.

(5) Bhalala, O. G.; Srikanth, M.; Kessler, J. A. The Emerging Roles of microRNAs in CNS Injuries. Nat. Rev. Neurol. 2013, 9 (6), 328-339.

(6) Schmidt, C. E.; Leach, J. B. Neural Tissue Engineering: Strategies for Repair and Regeneration. Annu. Rev. Biomed. Eng. 2003, 5, 293-347.

(7) Platt, J. L.; Vercellotti, G. M.; Dalmasso, A. P.; Matas, A. J.; Bolman, R. M.; Najarian, J. S.; Bach, F. H. Transplantation of Discordant Xenografts a Review of Progress. Immunol. Today 1990, 11 (12), 450-456.

(8) Fawcett, J. W.; Asher, R. a. The Glial Scar and Central Nervous System Repair. Brain Res. Bull. 1999, 49 (6), 377-391.

(9) Valentini, R. F.; Vargo, T. G.; Gardella, J. A.; Aebischer, P. Electrically Charged 
Polymeric Substrates Enhance Nerve Fibre Outgrowth In Vitro. Biomaterials 1992, $13(3), 183-190$.

(10) Lee, J. Y.; Bashur, C. a.; Goldstein, A. S.; Schmidt, C. E. Polypyrrole-Coated Electrospun PLGA Nanofibers for Neural Tissue Applications. Biomaterials 2009, $30(26), 4325-4335$.

(11) Metallo, C. M.; Mohr, J. C.; Detzel, C. J.; DePablo, J. J.; VanWie, B. J.; Palecek, S. P. Engineering the Stem Cell Microenvironment. Biotechnol. Prog. 2007, 23 (1), $18-23$.

(12) Peyton, S. R.; Kim, P. D.; Ghajar, C. M.; Seliktar, D.; Putnam, A. J. The Effects of Matrix Stiffness and RhoA on the Phenotypic Plasticity of Smooth Muscle Cells in a 3-D Biosynthetic Hydrogel System. Biomaterials 2008, 29 (17), 2597-2607.

(13) Engler, A. J.; Griffin, M. A.; Sen, S.; Bönnemann, C. G.; Sweeney, H. L.; Discher, D. E. Myotubes Differentiate Optimally on Substrates with Tissue-like Stiffness. $J$. Cell Biol. 2004, $166(6), 877-887$.

(14) Cavalcanti-Adam, E. A.; Volberg, T.; Micoulet, A.; Kessler, H.; Geiger, B.; Spatz, J. P. Cell Spreading and Focal Adhesion Dynamics Are Regulated by Spacing of Integrin Ligands. Biophys. J. 2007, 92 (8), 2964-2974.

(15) Chen, C. S.; Alonso, J. L.; Ostuni, E.; Whitesides, G. M.; Ingber, D. E. Cell Shape Provides Global Control of Focal Adhesion Assembly. Biochem. Biophys. Res. Commun. 2003, 307 (2), 355-361.

(16) Arnsdorf, E. J.; Tummala, P.; Kwon, R. Y.; Jacobs, C. R. Mechanically Induced Osteogenic Differentiation - the Role of RhoA, ROCKII and Cytoskeletal 
Dynamics. J. Cell Sci. 2009, 122 (4), 546-553.

(17) Dalby, M. J. Topographically Induced Direct Cell Mechanotransduction. Med. Eng. Phys. 2005, 27 (9), 730-742.

(18) Thrivikraman, G.; Madras, G.; Basu, B. Intermittent Electrical Stimuli for Guidance of Human Mesenchymal Stem Cell Lineage Commitment towards Neural-like Cells on Electroconductive Substrates. Biomaterials 2014, 35 (24), $6219-6235$.

(19) Jun, S. B.; Hynd, M. R.; Smith, K. L.; Song, J. K.; Turner, J. N.; Shain, W.; Kim, S. J. Electrical Stimulation-Induced Cell Clustering in Cultured Neural Networks. Med. Biol. Eng. Comput. 2007, 45 (11), 1015-1021.

(20) Ghasemi-Mobarakeh, L.; Prabhakaran, M. P.; Morshed, M.; Nasr-Esfahani, M. H.; Ramakrishna, S. Electrical Stimulation of Nerve Cells Using Conductive Nanofibrous Scaffolds for Nerve Tissue Engineering. Tissue Eng. Part A 2009, 15 (11), 3605-3619.

(21) Lysaght, M. J.; Reyes, J. The Growth of Tissue Engineering. Tissue Eng. 2001, 7 (5), 485-493.

(22) Khademhosseini, A.; Vacanti, J. P.; Langer, R. Progress in Tissue Engineering. Sci. Am. 2009, 300 (5), 64-71.

(23) Marx, V. Tissue Engineering: Organs from the Lab. Nature 2015, 522 (7556), 373 377.

(24) Barrilleaux, B.; Phinney, D. G.; Prockop, D. J.; O’Connor, K. C. Review: Ex Vivo Engineering of Living Tissues with Adult Stem Cells. Tissue Eng. 2006, 12 (11), 
$3007-3019$.

(25) Blau, H. M.; Brazelton, T. R.; Weimann, J. M. The Evolving Concept of a Stem Cell: Entity or Function? Cell 2001, 105 (7), 829-841.

(26) Kshitiz; Park, J.; Kim, P.; Helen, W.; Engler, A. J.; Levchenko, A.; Kim, D.-H. Control of Stem Cell Fate and Function by Engineering Physical Microenvironments. Integr. Biol. 2012, 4 (9), 1008.

(27) Friedenstein, A. J.; Petrakova, K. V; Kurolesova, A. I.; Frolova, G. P. Heterotopic of Bone Marrow. Analysis of Precursor Cells for Osteogenic and Hematopoietic Tissues. Transplantation 1968, 6 (2), 230-247.

(28) Lazarus, H. M.; Haynesworth, S. E.; Gerson, S. L.; Rosenthal, N. S.; Caplan, A. I. Ex Vivo Expansion and Subsequent Infusion of Human Bone Marrow-Derived Stromal Progenitor Cells (Mesenchymal Progenitor Cells): Implications for Therapeutic Use. Bone Marrow Transplant. 1995, 16 (4), 557-564.

(29) Chalfoun, C. T.; Wirth, G. A.; Evans, G. R. D. Tissue Engineered Nerve Constructs: Where Do We Stand? J. Cell. Mol. Med. 2006, 10 (2), 309-317.

(30) Gu, X. Progress and Perspectives of Neural Tissue Engineering. Front. Med. 2015, $9(4), 401-411$.

(31) Deumens, R.; Bozkurt, A.; Meek, M. F.; Marcus, M. A. E.; Joosten, E. A. J.; Weis, J.; Brook, G. A. Repairing Injured Peripheral Nerves: Bridging the Gap. Prog. Neurobiol. 2010, 92 (3), 245-276.

(32) Gu, X.; Ding, F.; Williams, D. F. Neural Tissue Engineering Options for Peripheral Nerve Regeneration. Biomaterials 2014, 35 (24), 6143-6156. 
(33) Gu, X.; Ding, F.; Yang, Y.; Liu, J. Construction of Tissue Engineered Nerve Grafts and Their Application in Peripheral Nerve Regeneration. Prog. Neurobiol. 2011, 93 (2), 204-230.

(34) Jiang, X.; Lim, S. H.; Mao Hai-Quan, H. Q.; Chew, S. Y. Current Applications and Future Perspectives of Artificial Nerve Conduits. Exp. Neurol. 2010, 223 (1), 86101.

(35) Khaing, Z. Z.; Schmidt, C. E. Advances in Natural Biomaterials for Nerve Tissue Repair. Neurosci. Lett. 2012, 519 (2), 103-114.

(36) Bianco, P.; Robey, P. G. Stem Cells in Tissue Engineering. Nature 2001, 414 (6859), 118-121.

(37) Ma, Z.; Kotaki, M.; Inai, R.; Ramakrishna, S. Potential of Nanofiber Matrix as Tissue-Engineering Scaffolds. Tissue Eng. 2005, 11 (1-2), 101-109.

(38) Nguyen, K. T.; West, J. L. Photopolymerizable Hydrogels for Tissue Engineering Applications. Biomaterials 2002, 23 (22), 4307-4314.

(39) Yang, S.; Leong, K. F.; Du, Z.; Chua, C. K. The Design of Scaffolds for Use in Tissue Engineering. Part I. Traditional Factors. Tissue Eng. 2001, 7 (6), 679-689.

(40) Yang, S.; Leong, K.-F.; Du, Z.; Chua, C.-K. The Design of Scaffolds for Use in Tissue Engineering. Part II. Rapid Prototyping Techniques. Tissue Eng. 2002, 8 (1), $1-11$.

(41) Caplan, A. I. Adult Mesenchymal Stem Cells for Tissue Engineering versus Regenerative Medicine. J. Cell. Physiol. 2007, 213 (2), 341-347.

(42) Balint, R.; Cassidy, N. J.; Cartmell, S. H. Electrical Stimulation: A Novel Tool for 
Tissue Engineering. Tissue Eng. Part B-Reviews 2013, 19 (1), $48-57$.

(43) Thomson, J.; Itskovitz-Eldor, J.; Shapiro, S.; Waknitz, M.; Swiergiel, J.; Marshall, V.; Jones, J. Embryonic Stem Cell Lines Derived from Human Blastocysts. Science 1998, 282 (5391), 1145-1147.

(44) Sharma, R. R.; Pollock, K.; Hubel, A.; McKenna, D. Mesenchymal Stem or Stromal Cells: A Review of Clinical Applications and Manufacturing Practices. Transfusion 2014, 54 (5), 1418-1437.

(45) Martins, R. S.; Bastos, D.; Siqueira, M. G.; Heise, C. O.; Teixeira, M. J. Traumatic Injuries of Peripheral Nerves: A Review with Emphasis on Surgical Indication. Arq. Neuropsiquiatr. 2013, 71 (10), 811-814.

(46) Taylor, C. A.; Braza, D.; Rice, J. B.; Dillingham, T. The Incidence of Peripheral Nerve Injury in Extremity Trauma. Am. J. Phys. Med. Rehabil. 2008, 87 (5), 381385.

(47) Battiston, B.; Papalia, I.; Tos, P.; Geuna, S. Chapter 1 Peripheral Nerve Repair and Regeneration Research. A Historical Note, 1st ed.; Elsevier Inc., 2009; Vol. 87.

(48) Fitch, M. T.; Silver, J. CNS Injury, Glial Scars, and Inflammation: Inhibitory Extracellular Matrices and Regeneration Failure. Exp. Neurol. 2008, 209 (2), 294301.

(49) Filbin, M. T. Myelin-Associated Inhibitors of Axonal Regeneration in the Adult Mammalian CNS. Nat. Rev. Neurosci. 2003, 4 (9), 703-713.

(50) Artico, M.; Cervoni, L.; Nucci, F.; Giuffre, R. Birthday of Peripheral Nervous System Surgery: The Contribution of Gabriele Ferrara (1543-1627). Neurosurgery 
1996, 39 (2), 380-383.

(51) Bao, G.; Suresh, S. Cell and Molecular Mechanics of Biological Materials. Nat. Mater. 2003, 2 (11), 715-725.

(52) Tee, S.-Y.; Fu, J.; Chen, C. S.; Janmey, P. a. Cell Shape and Substrate Rigidity Both Regulate Cell Stiffness. Biophys. J. 2011, 100 (5), L25-L27.

(53) Park, J. S.; Chu, J. S. F.; Cheng, C.; Chen, F.; Chen, D.; Li, S. Differential Effects of Equiaxial and Uniaxial Strain on Mesenchymal Stem Cells. Biotechnol. Bioeng. 2004, 88 (3), 359-368.

(54) Park, S.-H. S. R. S. W.; Sim, W. Y.; Park, S.-H. S. R. S. W.; Yang, S. S.; Choi, B. H.; Park, S.-H. S. R. S. W.; Park, K.; Min, B.-H. An Electromagnetic Compressive Force by Cell Exciter Stimulates Chondrogenic Differentiation of Bone Marrow-Derived Mesenchymal Stem Cells. Tissue Eng. 2006, 12 (11), 3107-3117.

(55) Schaffer. Engineering Microenvironments to Control Stem Cell Fate and Function. StemBook 2008, 1-12.

(56) Yu, L. M. Y.; Leipzig, N. D.; Shoichet, M. S. Promoting Neuron Adhesion and Growth. Mater. Today 2008, 11 (5), 36-43.

(57) Seuntjens, E.; Umans, L.; Zwijsen, A.; Sampaolesi, M.; Verfaillie, C. M.; Huylebroeck, D. Transforming Growth Factor Type Beta and Smad Family Signaling in Stem Cell Function. Cytokine Growth Factor Rev. 2009, 20 (5-6), $449-458$.

(58) Takahashi, K.; Yamanaka, S. Induction of Pluripotent Stem Cells from Mouse Embryonic and Adult Fibroblast Cultures by Defined Factors. Cell 2006, 126 (4), 
$663-676$.

(59) Guilak, F.; Cohen, D. M.; Estes, B. T.; Gimble, J. M.; Liedtke, W.; Chen, C. S. Control of Stem Cell Fate by Physical Interactions with the Extracellular Matrix. Cell Stem Cell 2009, 5 (1), 17-26.

(60) Discher, D. E.; Janmey, P.; Wang, Y.-L. Tissue Cells Feel and Respond to the Stiffness of Their Substrate. Science 2005, 310 (5751), 1139-1143.

(61) Pelham, R.; Wang, Y.-L.; Robert J. Pelham, J.; Wang, Y.-L.; Pelham, R.; Wang, Y.-L. Cell Locomotion and Focal Adhesions Are Regulated by Substrate Flexibility. Proc. Natl. Acad. Sci. U. S. A. 1997, 94, 13661-13665.

(62) Tsuruma, A.; Tanaka, M.; Yamamoto, S.; Fukushima, N.; Yabu, H.; Shimomura, M. Topographical Control of Neurite Extension on Stripe-Patterned Polymer Films. Colloids Surfaces A Physicochem. Eng. Asp. 2006, 284-285, 470-474.

(63) Yu, T. T.; Shoichet, M. S. Guided Cell Adhesion and Outgrowth in Peptide-Modified Channels for Neural Tissue Engineering. Biomaterials 2005, 26 (13), 1507-1514.

(64) Bian, L.; Zhai, D. Y.; Zhang, E. C.; Mauck, R. L.; Burdick, J. a. Dynamic Compressive Loading Enhances Cartilage Matrix Synthesis and Distribution and Suppresses Hypertrophy in hMSC-Laden Hyaluronic Acid Hydrogels. Tissue Eng. Part A 2012, 18 (7-8), 715-724.

(65) Hardy, J. G.; Villancio-Wolter, M. K.; Sukhavasi, R. C.; Mouser, D. J.; Aguilar, D.; Geissler, S. a.; Kaplan, D. L.; Schmidt, C. E. Electrical Stimulation of Human Mesenchymal Stem Cells on Conductive Nanofibers Enhances Their 
Differentiation toward Osteogenic Outcomes. Macromol. Rapid Commun. 2015, 36 (21), 1884-1890.

(66) Pouille, P.-A.; Ahmadi, P.; Brunet, A.-C.; Farge, E. Mechanical Signals Trigger Myosin II Redistribution and Mesoderm Invagination in Drosophila Embryos. Sci. Signal. 2009, 2 (66), ra16.

(67) DuFort, C. C.; Paszek, M. J.; Weaver, V. M. Balancing Forces: Architectural Control of Mechanotransduction. Nat. Rev. Mol. Cell Biol. 2011, 12 (5), 308-319.

(68) Wei, S. C.; Fattet, L.; Tsai, J. H.; Guo, Y.; Pai, V. H.; Majeski, H. E.; Chen, A. C.; Sah, R. L.; Taylor, S. S.; Engler, A. J.; Yang, J. Matrix Stiffness Drives Epithelial-Mesenchymal Transition and Tumour Metastasis through a TWIST1-G3BP2 Mechanotransduction Pathway. Nat. Cell Biol. 2015, 17 (5), 678688.

(69) Paszek, M. J.; Zahir, N.; Johnson, K. R.; Lakins, J. N.; Rozenberg, G. I.; Gefen, A.; Reinhart-King, C. A.; Margulies, S. S.; Dembo, M.; Boettiger, D.; Hammer, D. A.; Weaver, V. M. Tensional Homeostasis and the Malignant Phenotype. Cancer Cell 2005, $8(3), 241-254$.

(70) Engler, A. J.; Sen, S.; Sweeney, H. L.; Discher, D. E. Matrix Elasticity Directs Stem Cell Lineage Specification. Cell 2006, 126 (4), 677-689.

(71) Gilbert, P. M.; Havenstrite, K. L.; Magnusson, K. E. G.; Sacco, A.; Leonardi, N. a; Kraft, P.; Nguyen, N. K.; Thrun, S.; Lutolf, M. P.; Blau, H. M. Substrate Elasticity Regulates Skeletal Muscle Stem Cell Self-Renewal in Culture. (Supplementary). Science 2010, 329 (5995), 1078-1081. 
(72) Yang, C.; Tibbitt, M. W.; Basta, L.; Anseth, K. S. Mechanical Memory and Dosing Influence Stem Cell Fate. Nat. Mater. 2014, 13 (6), 645-652.

(73) Matsuoka, F.; Takeuchi, I.; Agata, H.; Kagami, H.; Shiono, H.; Kiyota, Y.; Honda, H.; Kato, R. Morphology-Based Prediction of Osteogenic Differentiation Potential of Human Mesenchymal Stem Cells. PLoS One 2013, 8 (2).

(74) Khetan, S.; Guvendiren, M.; Legant, W. R.; Cohen, D. M.; Chen, C. S.; Burdick, J. a. Degradation-Mediated Cellular Traction Directs Stem Cell Fate in Covalently Crosslinked Three-Dimensional Hydrogels. Nat. Mater. 2013, 12 (5), 458-465.

(75) Huebsch, N.; Arany, P. R.; Mao, A. S.; Shvartsman, D.; Ali, O. A.; Bencherif, S. A.; Rivera-Feliciano, J.; Mooney, D. J. Harnessing Traction-Mediated Manipulation of the Cell/matrix Interface to Control Stem-Cell Fate. Nat. Mater. 2010, 9 (6), 518526.

(76) Trappmann, B.; Gautrot, J. E.; Connelly, J. T.; Strange, D. G. T.; Li, Y.; Oyen, M. L.; Cohen Stuart, M. a.; Boehm, H.; Li, B.; Vogel, V.; Spatz, J. P.; Watt, F. M.; Huck, W. T. S. Extracellular-Matrix Tethering Regulates Stem-Cell Fate. Nat. Mater. 2012, $11(8), 742-742$.

(77) Wen, J. H.; Vincent, L. G.; Fuhrmann, A.; Choi, Y. S.; Hribar, K. C.; Taylor-Weiner, H.; Chen, S.; Engler, A. J. Interplay of Matrix Stiffness and Protein Tethering in Stem Cell Differentiation. Nat. Mater. 2014, advance on (October), 1-21.

(78) HARRISON, R. G. ON THE STEREOTROPISM OF EMBRYONIC CELLS. Science (80-. ). 1911, 34 (870), 279-281.

(79) Weiss, P.; Garber, B. Shape and Movement of Mesenchyme Cells as Functions of 
the Physical Structure of the Medium: Contributions to a Quantitative Morphology. Proc. Natl. Acad. Sci. 1952, 38 (3), 264-280.

(80) Dalby, M. J. Cellular Response to Low Adhesion Nanotopographies. Int. J. Nanomedicine 2007, 2 (3), 373-381.

(81) Wilkinson, C. D. W.; Dalby, M.; Curtis, A. S. G. Making Structures for Cell Engineering. Eur. Cells Mater. 2004, 8, 21-26.

(82) Putnam, A. J.; Schultz, K.; Mooney, D. J. Control of Microtubule Assembly by Extracellular Matrix and Externally Applied Strain. Am. J. Physiol. Cell Physiol. 2001, 280 (3), C556-C564.

(83) Bettinger, C. J.; Langer, R.; Borenstein, J. T. Engineering Substrate Topography at the Micro- and Nanoscale to Control Cell Function. Angew. Chemie - Int. Ed. 2009, $48(30), 5406-5415$.

(84) Joergensen, N. L.; Le, D. Q. S.; Andersen, O. Z.; Foss, M.; Danielsen, C. C.; Foldager, C. B.; Lind, M.; Lysdahl, H. Topography-Guided Proliferation: Distinct Surface Microtopography Increases Proliferation of Chondrocytes In Vitro. Tissue Eng. Part A 2015, 21, ten.tea.2014.0697.

(85) Baker, R. M.; Yang, P.; Henderson, J. H.; Mather, P. T. Wrinkle Formation on a Biocompatible Shape Memory Polymer. 2011 IEEE 37th Annu. Northeast Bioeng. Conf. NEBEC 20112011.

(86) Curtis, A. S. G.; Wilkinson, C. D. W. Reactions of Cells to Topography. J. Biomater. Sci. Polym. Ed. 1998, 9 (12), 1313-1329.

(87) Dalby, M. J.; Riehle, M. O.; Johnstone, H.; Affrossman, S.; Curtis, A. S. G. In Vitro 
Reaction of Endothelial Cells to Polymer Demixed Nanotopography. Biomaterials 2002, 23 (14), 2945-2954.

(88) Curtis, A. S. G.; Casey, B.; Gallagher, J. O.; Pasqui, D.; Wood, M. A.; Wilkinson, C. D. W. Substratum Nanotopography and the Adhesion of Biological Cells. Are Symmetry or Regularity of Nanotopography Important? Biophys. Chem. 2001, 94 (3), 275-283.

(89) Gallagher, J. O.; McGhee, K. F.; Wilkinson, C. D. W.; Riehle, M. O. Interaction of Animal Cells with Ordered Nanotopography. IEEE Trans. Nanobioscience 2002, 1 (1), 24-28.

(90) Ginger, D. S.; Zhang, H.; Mirkin, C. A. The Evolution of Dip-Pen Nanolithography. Angew. Chemie - Int. Ed. 2004, 43 (1), 30-45.

(91) Curran, J. M.; Stokes, R.; Irvine, E.; Graham, D.; Amro, N. a; Sanedrin, R. G.; Jamil, H.; Hunt, J. a. Introducing Dip Pen Nanolithography as a Tool for Controlling Stem Cell Behaviour: Unlocking the Potential of the next Generation of Smart Materials in Regenerative Medicine. Lab Chip 2010, 10 (13), 1662.

(92) Gadegaard, N.; Thoms, S.; Macintyre, D. S.; Mcghee, K.; Gallagher, J.; Casey, B.; Wilkinson, C. D. W. Arrays of Nano-Dots for Cellular Engineering. Microelectron. Eng. 2003, 67-68, 162-168.

(93) Leven, R. M.; Virdi, A. S.; Sumner, D. R. Patterns of Gene Expression in Rat Bone Marrow Stromal Cells Cultured on Titanium Alloy Discs of Different Roughness. $J$. Biomed. Mater. Res. A 2004, 70, 391-401.

(94) Clark, P.; Connolly, P.; Curtis, a S.; Dow, J. a; Wilkinson, C. D. Topographical 
Control of Cell Behaviour. I. Simple Step Cues. Development 1987, 99 (3), 439448.

(95) Clark, P.; Connolly, P.; Curtis, A. S. G.; Dow, J. A. T.; Wilkinson, C. D. W. Topographical Control of Cell Behavior .2. Multiple Grooved Substrata. Development 1990, 108 (4), 635-644.

(96) Dalby, M. J.; Yarwood, S. J.; Riehle, M. O.; Johnstone, H. J. H.; Affrossman, S.; Curtis, A. S. G. Increasing Fibroblast Response to Materials Using Nanotopography: Morphological and Genetic Measurements of Cell Response to 13-Nm-High Polymer Demixed Islands. Exp. Cell Res. 2002, 276 (1), 1-9.

(97) Gomez, N.; Lu, Y.; Chen, S.; Schmidt, C. E. Immobilized Nerve Growth Factor and Microtopography Have Distinct Effects on Polarization versus Axon Elongation in Hippocampal Cells in Culture. Biomaterials 2007, 28 (2), 271-284.

(98) Johansson, F.; Carlberg, P.; Danielsen, N.; Montelius, L.; Kanje, M. Axonal Outgrowth on Nano-Imprinted Patterns. Biomaterials 2006, 27 (8), 1251-1258.

(99) Li, N.; Folch, A. Integration of Topographical and Biochemical Cues by Axons during Growth on Microfabricated 3-D Substrates. Exp. Cell Res. 2005, 311 (2), $307-316$.

(100) Foley, J. D.; Grunwald, E. W.; Nealey, P. F.; Murphy, C. J. Cooperative Modulation of Neuritogenesis by PC12 Cells by Topography and Nerve Growth Factor. Biomaterials 2005, 26 (17), 3639-3644.

(101) Mahoney, M. J.; Chen, R. R.; Tan, J.; Mark Saltzman, W. The Influence of Microchannels on Neurite Growth and Architecture. Biomaterials 2005, 26 (7), 
$771-778$

(102) Dalby, M. J.; Gadegaard, N.; Tare, R.; Andar, A.; Riehle, M. O.; Herzyk, P.; Wilkinson, C. D.; Oreffo, R. O. The Control of Human Mesenchymal Cell Differentiation Using Nanoscale Symmetry and Disorder. Nat. Mater. 2007, 6 (5), $407-413$.

(103) Yim, E. K. F.; Pang, S. W.; Leong, K. W. Synthetic Nanostructures Inducing Differentiation of Human Mesenchymal Stem Cells into Neuronal Lineage. Exp. Cell Res. 2007, 313 (9), 1820-1829.

(104) Elsdale, T.; Bard, J. COLLAGEN SUBSTRATA FOR STUDIES ON CELL BEHAVIOR. J. Cell Biol. 1972, 54 (3), 626-637.

(105) Barnes, C. P.; Sell, S. A.; Boland, E. D.; Simpson, D. G.; Bowlin, G. L. Nanofiber Technology: Designing the next Generation of Tissue Engineering Scaffolds. Adv. Drug Deliv. Rev. 2007, 59 (14), 1413-1433.

(106) Smith, L. A.; Ma, P. X. Nano-Fibrous Scaffolds for Tissue Engineering. Colloids Surfaces B Biointerfaces 2004, 39 (3), 125-131.

(107) Yang, F.; Murugan, R.; Wang, S.; Ramakrishna, S. Electrospinning of Nano/micro Scale Poly(1-Lactic Acid) Aligned Fibers and Their Potential in Neural Tissue Engineering. Biomaterials 2005, 26 (15), 2603-2610.

(108) Corey, J. M.; Lin, D. Y.; Mycek, K. B.; Chen, Q.; Samuel, S.; Feldman, E. L.; Martin, D. C. Aligned Electrospun Nanofibers Specify the Direction of Dorsal Root Ganglia Neurite Growth. J. Biomed. Mater. Res. A 2007, 83 (3), 636-645.

(109) Shaw, D.; Shoichet, M. S. Toward Spinal Cord Injury Repair Strategies: Peptide 
Surface Modification of Expanded Poly(tetrafluoroethylene) Fibers for Guided Neurite Outgrowth in Vitro. J. Craniofac. Surg. 2003, 14 (3), 308-316.

(110) Wen, X.; Tresco, P. A. Effect of Filament Diameter and Extracellular Matrix Molecule Precoating on Neurite Outgrowth and Schwann Cell Behavior on Multifilament Entubulation Bridging Device in Vitro. J. Biomed. Mater. Res. - Part A 2006, $76(3), 626-637$.

(111) Bellamkonda, R. V. Peripheral Nerve Regeneration: An Opinion on Channels, Scaffolds and Anisotropy. Biomaterials 2006, 27 (19), 3515-3518.

(112) Lee, J.; Cuddihy, M. J.; Kotov, N. A. Three-Dimensional Cell Culture Matrices: State of the Art. Tissue Eng Part B Rev. 2008, 14 (1), 61-86.

(113) Jeong, S. I.; Kwon, J. H.; Lim, J. I.; Cho, S. W.; Jung, Y.; Sung, W. J.; Kim, S. H.; Kim, Y. H.; Lee, Y. M.; Kim, B. S.; Choi, C. Y.; Kim, S. J. Mechano-Active Tissue Engineering of Vascular Smooth Muscle Using Pulsatile Perfusion Bioreactors and Elastic PLCL Scaffolds. Biomaterials 2005, 26 (12), 1405-1411.

(114) Saha, S.; Ji, L.; De Pablo, J. J.; Palecek, S. P. Inhibition of Human Embryonic Stem Cell Differentiation by Mechanical Strain. J. Cell. Physiol. 2006, 206 (1), 126-137.

(115) Saha, S.; Ji, L.; de Pablo, J. J.; Palecek, S. P. TGFbeta/Activin/Nodal Pathway in Inhibition of Human Embryonic Stem Cell Differentiation by Mechanical Strain. Biophys. J. 2008, 94 (10), 4123-4133.

(116) Simmons, C. A.; Matlis, S.; Thornton, A. J.; Chen, S.; Wang, C. Y.; Mooney, D. J. Cyclic Strain Enhances Matrix Mineralization by Adult Human Mesenchymal Stem Cells via the Extracellular Signal-Regulated Kinase (ERK1/2) Signaling 
Pathway. J. Biomech. 2003, 36 (8), 1087-1096.

(117) GH, A.; RL, H.; I, M.; J, F.; PR, S.; V, V.; JC, R.; G, V.-N.; DL, K. Cell Differentiation by Mechanical Stress. FASEB J. 2001, 16 (13), 1805-1807.

(118) Shi, G.; Zhang, Z.; Rouabhia, M. The Regulation of Cell Functions Electrically Using Biodegradable Polypyrrole-Polylactide Conductors. Biomaterials 2008, 29 (28), 3792-3798.

(119) Rowlands, A. S.; Cooper-White, J. J. Directing Phenotype of Vascular Smooth Muscle Cells Using Electrically Stimulated Conducting Polymer. Biomaterials 2008, $29(34), 4510-4520$.

(120) Kim, I. S.; Song, J. K.; Song, Y. M.; Cho, T. H.; Lee, T. H.; Lim, S. S.; Kim, S. J.; Hwang, S. J. Novel Effect of Biphasic Electric Current on in Vitro Osteogenesis and Cytokine Production in Human Mesenchymal Stromal Cells. Tissue Eng. Part A 2009, 15 (9), 2411-2422.

(121) Sundelacruz, S.; Levin, M.; Kaplan, D. L. Membrane Potential Controls Adipogenic and Osteogenic Differentiation of Mesenchymal Stem Cells. PLoS One 2008, $3(11), 1-15$.

(122) Brighton, C. T.; Wang, W.; Seldes, R.; Zhang, G.; Pollack, S. R. Signal Transduction in Electrically Stimulated Bone Cells. J. Bone Joint Surg. Am. 2001, $83-A(10), 1514-1523$.

(123) Hammerick, K. E.; Longaker, M. T.; Prinz, F. B. In Vitro Effects of Direct Current Electric Fields on Adipose-Derived Stromal Cells. Biochem. Biophys. Res. Commun. 2010, 397 (1), 12-17. 
(124) Wang, E.; Zhao, M.; Forrester, J. V.; McCaig, C. D. Bi-Directional Migration of Lens Epithelial Cells in a Physiological Electrical Field. Exp. Eye Res. 2003, 76 (1), $29-37$.

(125) Wang, E.; Zhao, M.; Forrester, J. V; MCCaig, C. D. Re-Orientation and Faster, Directed Migration of Lens Epithelial Cells in a Physiological Electric Field. Exp. Eye Res. 2000, 71 (1), 91-98.

(126) Sun, S.; Titushkin, I.; Cho, M. Regulation of Mesenchymal Stem Cell Adhesion and Orientation in 3D Collagen Scaffold by Electrical Stimulus. Bioelectrochemistry 2006, 69 (2), 133-141.

(127) Mendonça, A. C.; Barbieri, C. H.; Mazzer, N. Directly Applied Low Intensity Direct Electric Current Enhances Peripheral Nerve Regeneration in Rats. $J$. Neurosci. Methods 2003, 129 (2), 183-190.

(128) Kim, I. S.; Song, J. K.; Zhang, Y. L.; Lee, T. H.; Cho, T. H.; Song, Y. M.; Kim, D. K.; Kim, S. J.; Hwang, S. J. Biphasic Electric Current Stimulates Proliferation and Induces VEGF Production in Osteoblasts. Biochim. Biophys. Acta - Mol. Cell Res. 2006, 1763 (9), 907-916.

(129) Ross, C. L.; Siriwardane, M.; Almeida-Porada, G.; Porada, C. D.; Brink, P.; Christ, G. J.; Harrison, B. S. The Effect of Low-Frequency Electromagnetic Field on Human Bone Marrow Stem/progenitor Cell Differentiation. Stem Cell Res. 2015, $15(1), 96-108$.

(130) Bouaziz, A.; Richert, A.; Caprani, A. Morphological Aspects of Endothelial Cells Cultured in the Absence of Fetal Calf Serum under Controlled Electrical Charges 
Applied to the Support. Biomaterials 1996, 17 (23), 2281-2287.

(131) Hartig, M.; Joos, U.; Wiesmann, H. P. Capacitively Coupled Electric Fields Accelerate Proliferation of Osteoblast-like Primary Cells and Increase Bone Extracellular Matrix Formation in Vitro. Eur. Biophys. J. 2000, 29 (7), 499-506.

(132) Lohmann, C. H.; Schwartz, Z.; Liu, Y.; Li, Z.; Simon, B. J.; Sylvia, V. L.; Dean, D. D.; Bonewald, L. F.; Donahue, H. J.; Boyan, B. D. Pulsed Electromagnetic Fields Affect Phenotype and Connexin 43 Protein Expression in MLO-Y4 Osteocyte-like Cells and ROS 17/2.8 Osteoblast-like Cells. J. Orthop. Res. 2003, 21 (2), 326-334.

(133) Chang, K.; Chang, W. H. S.; Huang, S.; Huang, S.; Shih, C. Pulsed Electromagnetic Fields Stimulation Affects Osteoclast Formation by Modulation of Osteoprotegerin, RANK Ligand and Macrophage Colony-Stimulating Factor. $J$. Orthop. Res. 2005, 23 (6), 1308-1314.

(134) Serena, E.; Figallo, E.; Tandon, N.; Cannizzaro, C.; Gerecht, S.; Elvassore, N.; Vunjak-Novakovic, G. Electrical Stimulation of Human Embryonic Stem Cells: Cardiac Differentiation and the Generation of Reactive Oxygen Species. Exp. Cell Res. 2009, 315 (20), 3611-3619.

(135) Lam, H.; Qin, Y. X. The Effects of Frequency-Dependent Dynamic Muscle Stimulation on Inhibition of Trabecular Bone Loss in a Disuse Model. Bone 2008, $43(6), 1093-1100$.

(136) Aaron, R. K.; Wang, S.; Ciombor, D. M. K. Upregulation of Basal TGF??1 Levels by EMF Coincident with Chondrogenesis - Implications for Skeletal Repair and Tissue Engineering. J. Orthop. Res. 2002, 20 (2), 233-240. 
(137) Kim, I. S.; Song, J. K.; Song, Y. M.; Lee, T. H.; Cho, T. H.; Lim, S. S.; Pan, H.; Kim, S. J.; Hwang, S. J. Novel Action of Biphasic Electric Current in Vitro Osteogenesis of Human Bone Marrow Mesenchymal Stromal Cells Coupled with VEGF Production. Bone 2008, 43 (2008), S43-S44.

(138) Petersson, C. J.; Holmer, N. G.; Johnell, O. Electrical Stimulation of Osteogenesis: Studies of the Cathode Effect on Rabbit Femur. Acta Orthop Scand 1982, 53 (5), $727-732$.

(139) Ercan, B.; Webster, T. J. The Effect of Biphasic Electrical Stimulation on Osteoblast Function at Anodized Nanotubular Titanium Surfaces. Biomaterials 2010, 31 (13), 3684-3693.

(140) Merrill, D. R.; Bikson, M.; Jefferys, J. G. R. Electrical Stimulation of Excitable Tissue: Design of Efficacious and Safe Protocols. J. Neurosci. Methods 2005, 141 (2), 171-198.

(141) Spadaro, J. A.; Becker, R. O. Function of Implanted Cathodes in Electrode-Induced Bone Growth. Med. Biol. Eng. Comput. 1979, 17 (6), 769-775.

(142) Zhuang, H.; Wang, W.; Seldes, R. M.; Tahernia, A. D.; Fan, H.; Brighton, C. T. Electrical Stimulation Induces the Level of TGF- $\beta 1$ mRNA in Osteoblastic Cells by a Mechanism Involving Calcium/Calmodulin Pathway. Biochem. Biophys. Res. Commun. 1997, 237 (2), 225-229.

(143) Antov, Y.; Barbul, A.; Mantsur, H.; Korenstein, R. Electroendocytosis: Exposure of Cells to Pulsed Low Electric Fields Enhances Adsorption and Uptake of Macromolecules. Biophys. J. 2005, 88 (March), 2206-2223. 
(144) Xu, J.; Wang, W.; Clark, C. C.; Brighton, C. T. Signal Transduction in Electrically Stimulated Articular Chondrocytes Involves Translocation of Extracellular Calcium through Voltage-Gated Channels. Osteoarthr. Cartil. 2009, 17 (3), 397-405.

(145) Huang, C. P.; Chen, X. M.; Chen, Z. Q. Osteocyte: The Impresario in the Electrical Stimulation for Bone Fracture Healing. Med. Hypotheses 2008, 70 (2), 287-290.

(146) Au, H. T. H.; Cheng, I.; Chowdhury, M. F.; Radisic, M. Interactive Effects of Surface Topography and Pulsatile Electrical Field Stimulation on Orientation and Elongation of Fibroblasts and Cardiomyocytes. Biomaterials 2007, 28 (29), 42774293.

(147) Zhao, M. Electrical Fields in Wound Healing-An Overriding Signal That Directs Cell Migration. Semin. Cell Dev. Biol. 2009, 20 (6), 674-682.

(148) Mycielska, M. E.; Djamgoz, M. B. a. Cellular Mechanisms of Direct-Current Electric Field Effects: Galvanotaxis and Metastatic Disease. J. Cell Sci. 2004, 117 (Pt 9), 1631-1639.

(149) Becker, D.; Gary, D. S.; Rosenzweig, E. S.; Grill, W. M.; McDonald, J. W. Functional Electrical Stimulation Helps Replenish Progenitor Cells in the Injured Spinal Cord of Adult Rats. Exp. Neurol. 2010, 222 (2), 211-218.

(150) Schmidt, C. E.; Shastri, V. R.; Vacanti, J. P.; Langer, R. Stimulation of Neurite Outgrowth Using an Electrically Conducting Polymer. Proc. Natl. Acad. Sci. U. S. A. 1997, 94 (17), 8948-8953.

(151) Wiesmann, H.-P.; Hartig, M.; Stratmann, U.; Meyer, U.; Joos, U. Electrical Stimulation Influences Mineral Formation of Osteoblast-like Cells in Vitro. 
Biochim. Biophys. Acta - Mol. Cell Res. 2001, 1538 (1), 28-37.

(152) Subbiah, T.; Bhat, G. S.; Tock, R. W.; Parameswaran, S.; Ramkumar, S. S. Electrospinning of Nanofibers. J. Appl. Polym. Sci. 2005, 96 (2), 557-569.

(153) Huang, Z. M.; Zhang, Y. Z.; Kotaki, M.; Ramakrishna, S. A Review on Polymer Nanofibers by Electrospinning and Their Applications in Nanocomposites. Compos. Sci. Technol. 2003, 63 (15), 2223-2253.

(154) Frenot, A.; Chronakis, I. S. Polymer Nanofibers Assembled by Electrospinning. Curr. Opin. Colloid Interface Sci. 2003, 8 (1), 64-75.

(155) Geng, X.; Kwon, O. H.; Jang, J. Electrospinning of Chitosan Dissolved in Concentrated Acetic Acid Solution. Biomaterials 2005, 26 (27), 5427-5432.

(156) Sill, T. J.; von Recum, H. A. Electrospinning: Applications in Drug Delivery and Tissue Engineering. Biomaterials 2008, 29 (13), 1989-2006.

(157) Zhao, P.; Jiang, H.; Pan, H.; Zhu, K.; Chen, W. Biodegradable Fibrous Scaffolds Composed of Gelatin Coated Poly( $\varepsilon$-Caprolactone) Prepared by Coaxial Electrospinning. J. Biomed. Mater. Res. Part A 2007, 83A (2), 372-382.

(158) Yang, D.-J.; Zhang, L.-F.; Xu, L.; Xiong, C.-D.; Ding, J.; Wang, Y.-Z. Fabrication and Characterization of Hydrophilic Electrospun Membranes Made from the Block Copolymer of Poly(ethylene Glycol-Co-Lactide). J. Biomed. Mater. Res. Part A 2007, $82 A(3), 680-688$.

(159) Schnell, E.; Klinkhammer, K.; Balzer, S.; Brook, G.; Klee, D.; Dalton, P.; Mey, J. Guidance of Glial Cell Migration and Axonal Growth on Electrospun Nanofibers

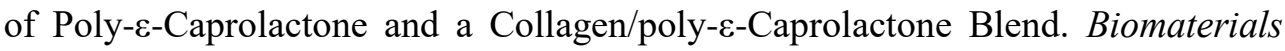


2007, 28 (19), 3012-3025.

(160) Park, K.; Ju, Y. M.; Son, J. S.; Ahn, K.-D.; Han, D. K. Surface Modification of Biodegradable Electrospun Nanofiber Scaffolds and Their Interaction with Fibroblasts. J. Biomater. Sci. Polym. Ed. 2007, 18 (March 2015), 369-382.

(161) Li, W. J.; Mauck, R. L.; Cooper, J. A.; Yuan, X.; Tuan, R. S. Engineering Controllable Anisotropy in Electrospun Biodegradable Nanofibrous Scaffolds for Musculoskeletal Tissue Engineering. J. Biomech. 2007, 40 (8), 1686-1693.

(162) Henry, J. A.; Simonet, M.; Pandit, A.; Neuenschwander, P. Characterization of a Slowly Degrading Biodegradable Polyesterurethane for Tissue Engineering Scaffolds. J. Biomed. Mater. Res. Part A 2007, 82A (3), 669-679.

(163) Duan, B.; Wu, L.; Li, X.; Yuan, X.; Li, X.; Zhang, Y.; Yao, K. Degradation of Electrospun PLGA-chitosan/PVA Membranes and Their Cytocompatibility in Vitro. J. Biomater. Sci. Polym. Ed. 2007, 18 (1), 95-115.

(164) Ashammakhi, N.; Ndreu, A.; Piras, A.; Nikkola, L.; Sindelar, T.; Ylikauppila, H.; Harlin, A.; Chiellini, E.; Hasirci, V.; Redl, H. Biodegradable Nanomats Produced by Electrospinning: Expanding Multifunctionality and Potential for Tissue Engineering. J. Nanosci. Nanotechnol. 2006, 6 (9), 2693-2711.

(165) Thomas, V.; Jose, M. V; Chowdhury, S.; Sullivan, J. F.; Dean, D. R.; Vohra, Y. K. Mechano-Morphological Studies of Aligned Nanofibrous Scaffolds of Polycaprolactone Fabricated by Electrospinning. J. Biomater. Sci. Polym. Ed. 2006, 17 (9), 969-984.

(166) Chew, S. Y.; Wen, J.; Yim, E. K. F.; Leong, K. W. Sustained Release of Proteins 
from Electrospun Biodegradable Fibers. Biomacromolecules 2005, 6 (4), 20172024.

(167) Zong, X.; Li, S.; Chen, E.; Garlick, B.; Kim, K.-S.; Fang, D.; Chiu, J.; Zimmerman, T.; Brathwaite, C.; Hsiao, B. S.; Chu, B. Prevention of Postsurgery-Induced Abdominal Adhesions by Electrospun Bioabsorbable Nanofibrous Poly(lactide-Co-Glycolide)-Based Membranes. Ann. Surg. 2004, 240 (5), 910-915.

(168) Xu, C. Y.; Inai, R.; Kotaki, M.; Ramakrishna, S. Aligned Biodegradable Nanofibrous Structure: A Potential Scaffold for Blood Vessel Engineering. Biomaterials 2004, 25 (5), 877-886.

(169) Kim, K.; Yu, M.; Zong, X.; Chiu, J.; Fang, D.; Seo, Y. S.; Hsiao, B. S.; Chu, B.; Hadjiargyrou, M. Control of Degradation Rate and Hydrophilicity in Electrospun Non-Woven poly(D,L-Lactide) Nanofiber Scaffolds for Biomedical Applications. Biomaterials 2003, 24 (27), 4977-4985.

(170) Li, W. J.; Laurencin, C. T.; Caterson, E. J.; Tuan, R. S.; Ko, F. K. Electrospun Nanofibrous Structure: A Novel Scaffold for Tissue Engineering. J. Biomed. Mater. Res. 2002, 60 (4), 613-621.

(171) Karim, M. R. Fabrication of Electrospun Aligned Nanofibers from Conducting Polyaniline Copolymer/polyvinyl Alcohol/chitosan Oligossacaride in Aqueous Solutions. Synth. Met. 2013, 178, 34-37.

(172) Kenawy, E. R.; Layman, J. M.; Watkins, J. R.; Bowlin, G. L.; Matthews, J. a; Simpson, D. G.; Wnek, G. E. Electrospinning of Poly(ethylene-Co-Vinyl Alcohol) Fibers. Biomaterials 2003, 24 (6), 907-913. 
(173) McManus, M. C.; Boland, E. D.; Simpson, D. G.; Barnes, C. P.; Bowlin, G. L. Electrospun Fibrinogen: Feasibility as a Tissue Engineering Scaffold in a Rat Cell Culture Model. J. Biomed. Mater. Res. Part A 2007, 81A (2), 299-309.

(174) Noh, H. K.; Lee, S. W.; Kim, J. M.; Oh, J. E.; Kim, K. H.; Chung, C. P.; Choi, S. C.; Park, W. H.; Min, B. M. Electrospinning of Chitin Nanofibers: Degradation Behavior and Cellular Response to Normal Human Keratinocytes and Fibroblasts. Biomaterials 2006, 27 (21), 3934-3944.

(175) Ji, Y.; Ghosh, K.; Shu, X. Z.; Li, B.; Sokolov, J. C.; Prestwich, G. D.; Clark, R. A. F.; Rafailovich, M. H. Electrospun Three-Dimensional Hyaluronic Acid Nanofibrous Scaffolds. Biomaterials 2006, 27 (20), 3782-3792.

(176) Matthews, J. A.; Wnek, G. E.; Simpson, D. G.; Bowlin, G. L. Electrospinning of Collagen Nanofibers. Biomacromolecules 2002, 3 (2), 232-238.

(177) Zhong, S.; Teo, W. E.; Zhu, X.; Beuerman, R. W.; Ramakrishna, S.; Yung, L. Y. L. An Aligned Nanofibrous Collagen Scaffold by Electrospinning and Its Effects Onin Vitro Fibroblast Culture. J. Biomed. Mater. Res. Part A 2006, 79 A (3), 456-463.

(178) Ayres, C.; Bowlin, G. L.; Henderson, S. C.; Taylor, L.; Shultz, J.; Alexander, J.; Telemeco, T. A.; Simpson, D. G. Modulation of Anisotropy in Electrospun Tissue-Engineering Scaffolds: Analysis of Fiber Alignment by the Fast Fourier Transform. Biomaterials 2006, 27 (32), 5524-5534.

(179) Song, J. H.; Kim, H. E.; Kim, H. W. Production of Electrospun Gelatin Nanofiber by Water-Based Co-Solvent Approach. J. Mater. Sci. Mater. Med. 2008, 19 (1), 95102. 
(180) Stitzel, J.; Liu, J.; Lee, S. J.; Komura, M.; Berry, J.; Soker, S.; Lim, G.; Van Dyke, M.; Czerw, R.; Yoo, J. J.; Atala, A. Controlled Fabrication of a Biological Vascular Substitute. Biomaterials 2006, 27 (7), 1088-1094.

(181) Sui, G.; Yang, X.; Mei, F.; Hu, X.; Chen, G.; Deng, X.; Ryu, S. Poly-L-Lactic Acid/hydroxyapatite Hybrid Membrane for Bone Tissue Regeneration. J. Biomed. Mater. Res. Part A 2007, 82A (2), 445-454.

(182) Nie, H.; Wang, C. H. Fabrication and Characterization of PLGA/HAp Composite Scaffolds for Delivery of BMP-2 Plasmid DNA. J. Control. Release 2007, 120 (12), 111-121.

(183) Mohammadi, Y.; Soleimani, M.; Fallahi-Sichani, M.; Gazme, a; Haddadi-Asl, V.; Arefian, E.; Kiani, J.; Moradi, R.; Atashi, a; Ahmadbeigi, N. Nanofibrous Poly(epsilon-Caprolactone)/poly(vinyl Alcohol)/chitosan Hybrid Scaffolds for Bone Tissue Engineering Using Mesenchymal Stem Cells. Int. J. Artif. Organs 2007, 30 (3), 204-211.

(184) Meng, W.; Kim, S.-Y.; Yuan, J.; Kim, J. C.; Kwon, O. H.; Kawazoe, N.; Chen, G.; Ito, Y.; Kang, I.-K. Electrospun PHBV/collagen Composite Nanofibrous Scaffolds for Tissue Engineering. J. Biomater. Sci. Polym. Ed. 2007, 18 (March 2015), 8194.

(185) Duan, B.; Wu, L.; Yuan, X.; Hu, Z.; Li, X.; Zhang, Y.; Yao, K.; Wang, M. Hybrid Nanofibrous Membranes of PLGA/chitosan Fabricated via an Electrospinning Array. J. Biomed. Mater. Res. Part A 2007, 83A (3), 868-878.

(186) Peesan, M.; Rujiravanit, R.; Supaphol, P. Electrospinning of Hexanoyl 
Chitosan/polylactide Blends. J. Biomater. Sci. Polym. Ed. 2006, 17 (5), 547-565.

(187) Park, K. E.; Kang, H. K.; Lee, S. J.; Min, B. M.; Park, W. H. Biomimetic Nanofibrous Scaffolds: Preparation and Characterization of PGA/chitin Blend Nanofibers. Biomacromolecules 2006, 7 (2), 635-643.

(188) Li, L.; Hsieh, Y. Lo. Chitosan Bicomponent Nanofibers and Nanoporous Fibers. Carbohydr. Res. 2006, 341 (3), 374-381.

(189) Huang, L.; Nagapudi, K.; Apkarian, R. P.; Chaikof, E. L. Engineered Collagen PEO Nanofibers and Fabrics. J Biomater Sci Polym. Edn 2001, 12 (9), 979-993.

(190) Courtney, T.; Sacks, M. S.; Stankus, J.; Guan, J.; Wagner, W. R. Design and Analysis of Tissue Engineering Scaffolds That Mimic Soft Tissue Mechanical Anisotropy. Biomaterials 2006, 27 (19), 3631-3638.

(191) Kakade, M. V.; Givens, S.; Gardner, K.; Lee, K. H.; Chase, D. B.; Rabolt, J. F. Electric Field Induced Orientation of Polymer Chains in Macroscopically Aligned Electrospun Polymer Nanofibers. J. Am. Chem. Soc. 2007, 129 (10), 2777-2782.

(192) Eichhorn, S. J.; Sampson, W. W. Statistical Geometry of Pores and Statistics of Porous Nanofibrous Assemblies. J. R. Soc. Interface 2005, 2 (4), 309-318.

(193) Nam, J.; Huang, Y.; Agarwal, S.; Lannutti, J. Improved Cellular Infiltration in Electrospun Fiber via Engineered Porosity. Tissue Eng. 2007, 13 (9), 2249-2257.

(194) Pham, Q. P.; Sharma, U.; Mikos, A. G. Electrospun Poly(ع-Caprolactone) Microfiber and Multilayer Nanofiber/Microfiber Scaffolds: Characterization of Scaffolds and Measurement of Cellular Infiltration. Biomacromolecules 2006, 7 (10), 2796-2805. 
(195) Kim, T. G.; Park, T. G. Biomimicking Extracellular Matrix: Cell Adhesive RGD Peptide Modified Electrospun Poly(D,L-Lactic-Co-Glycolic Acid) Nanofiber Mesh. Tissue Eng. 2006, 12 (2), 221-233.

(196) Ma, Z.; He, W.; Yong, T.; Ramakrishna, S. Grafting of Gelatin on Electrospun Poly(caprolactone) Nanofibers to Improve Endothelial Cell Spreading and Proliferation and to Control Cell Orientation. Tissue Eng. 2005, 11 (7-8), 11491158.

(197) Casper, C. L.; Yang, W.; Farach-Carson, M. C.; Rabolt, J. F. Coating Electrospun Collagen and Gelatin Fibers with Perlecan Domain I for Increased Growth Factor Binding. Biomacromolecules 2007, 8 (4), 1116-1123.

(198) Feng, Z.-Q.; Wang, T.; Zhao, B.; Li, J.; Jin, L. Soft Graphene Nanofibers Designed for the Acceleration of Nerve Growth and Development. Adv. Mater. 2015, 27 (41), $6462-6468$.

(199) Lee, S. J.; Yoo, J. J.; Lim, G. J.; Atala, A.; Stitzel, J. In Vitro Evaluation of Electrospun Nanofiber Scaffolds for Vascular Graft Application. J. Biomed. Mater. Res. Part A 2007, 83 A (4), 999-1008.

(200) Inoguchi, H.; Tanaka, T.; Maehara, Y.; Matsuda, T. The Effect of Gradually Graded Shear Stress on the Morphological Integrity of a Huvec-Seeded Compliant Small-Diameter Vascular Graft. Biomaterials 2007, 28 (3), 486-495.

(201) Zhang, J.; Qi, H.; Wang, H.; Hu, P.; Ou, L.; Guo, S.; Li, J.; Che, Y.; Yu, Y.; Kong, D. Engineering of Vascular Grafts with Genetically Modified Bone Marrow Mesenchymal Stem Cells on Poly (Propylene Carbonate) Graft. Artif. Organs 2006, 
30 (12), 898-905.

(202) Inoguchi, H.; Kwon, I. K.; Inoue, E.; Takamizawa, K.; Maehara, Y.; Matsuda, T. Mechanical Responses of a Compliant Electrospun poly(L-Lactide-Co- $\varepsilon$ Caprolactone) Small-Diameter Vascular Graft. Biomaterials 2006, 27 (8), 14701478.

(203) He, W.; Yong, T.; Teo, W. E.; Ma, Z.; Ramakrishna, S. Fabrication and Endothelialization of Collagen-Blended Biodegradable Polymer Nanofibers: Potential Vascular Graft for Blood Vessel Tissue Engineering. Tissue Eng. 2005, 11 (9), 1574-1588.

(204) Nie, H.; Soh, B. W.; Fu, Y.-C.; Wang, C.-H. Three-Dimensional Fibrous PLGA/HAp Composite Scaffold for BMP-2 Delivery. Biotechnol. Bioeng. 2008, 99 (1), 223-234.

(205) Cui, W.; Li, X.; Zhou, S.; Weng, J. In Situ Growth of Hydroxyapatite within Electrospun poly(DL-Lactide) Fibers. J. Biomed. Mater. Res. Part A 2007, 82A (4), 831-841.

(206) Burger, C.; Chu, B. Functional Nanofibrous Scaffolds for Bone Reconstruction. Colloids Surfaces B Biointerfaces 2007, 56 (1-2), 134-141.

(207) Sahoo, S.; Ouyang, H.; Goh, J. C.-H.; Tay, T. E.; Toh, S. L. Characterization of a Novel Polymeric Scaffold for Potential Application in Tendon/Ligament Tissue Engineering. Tissue Eng. 2006, 12 (1), 91-99.

(208) Lee, C. H.; Shin, H. J.; Cho, I. H.; Kang, Y. M.; Kim, I. A.; Park, K. D.; Shin, J. W. Nanofiber Alignment and Direction of Mechanical Strain Affect the ECM 
Production of Human ACL Fibroblast. Biomaterials 2005, 26 (11), 1261-1270.

(209) Giordano, A.; Galderisi, U. From the Laboratory Bench to the Patient' S Bedside : An Update on Clinical Trials with Mesenchymal Stem Cells. J. Cell. Physiol. 2007, 2 I I (October), 27-35.

(210) Lee, J.; Abdeen, A. A.; Kilian, K. A. Rewiring Mesenchymal Stem Cell Lineage Specification by Switching the Biophysical Microenvironment. Sci. Rep. 2014, 4, 5188.

(211) Kobayashi, N.; Yasu, T.; Ueba, H.; Sata, M.; Hashimoto, S.; Kuroki, M.; Saito, M.; Kawakami, M. Mechanical Stress Promotes the Expression of Smooth Muscle-like Properties in Marrow Stromal Cells. Exp. Hematol. 2004, 32 (12), 1238-1245.

(212) Chuah, Y. J.; Zhang, Y.; Wu, Y.; Menon, N. V.; Goh, G. H.; Lee, A. C.; Chan, V.; Zhang, Y.; Kang, Y. Combinatorial Effect of Substratum Properties on Mesenchymal Stem Cell Sheet Engineering and Subsequent Multi-Lineage Differentiation. Acta Biomater. 2015.

(213) Mullen, C. A.; Vaughan, T. J.; Billiar, K. L.; McNamara, L. M. The Effect of Substrate Stiffness, Thickness, and Cross-Linking Density on Osteogenic Cell Behavior. Biophys. J. 2015, 108 (7), 1604-1612.

(214) Lee, J.; Abdeen, A. a.; Zhang, D.; Kilian, K. a. Directing Stem Cell Fate on Hydrogel Substrates by Controlling Cell Geometry, Matrix Mechanics and Adhesion Ligand Composition. Biomaterials 2013, 34 (33), 8140-8148.

(215) Khani, M.-M.; Tafazzoli-Shadpour, M.; Rostami, M.; Peirovi, H.; Janmaleki, M. Evaluation of Mechanical Properties of Human Mesenchymal Stem Cells during 
Differentiation to Smooth Muscle Cells. Ann. Biomed. Eng. 2014, 42 (7), 13731380.

(216) Gonzalez-Cruz, R. D.; Fonseca, V. C.; Darling, E. M. Cellular Mechanical Properties Reflect the Differentiation Potential of Adipose-Derived Mesenchymal Stem Cells. Proc. Natl. Acad. Sci. 2012, 109 (24), E1523-E1529.

(217) Evans, E.; Yeung, a. Apparent Viscosity and Cortical Tension of Blood Granulocytes Determined by Micropipet Aspiration. Biophys. J. 1989, 56 (1), $151-$ 160.

(218) Hochmuth, R. M. Micropipette Aspiration of Living Cells. J. Biomech. 2000, 33 (1), 15-22.

(219) Oh, M.-J.; Kuhr, F.; Byfield, F.; Levitan, I. Micropipette Aspiration of Substrate-Attached Cells to Estimate Cell Stiffness. J. Vis. Exp. 2012, No. 67, 1-7.

(220) Shao, J. Y.; Hochmuth, R. M. Micropipette Suction for Measuring Piconewton Forces of Adhesion and Tether Formation from Neutrophil Membranes. Biophys. J. 1996, $71(5), 2892-2901$.

(221) Tan, S. C. W.; Pan, W. X.; Ma, G.; Cai, N.; Leong, K. W.; Liao, K. Viscoelastic Behaviour of Human Mesenchymal Stem Cells. BMC Cell Biol. 2008, 9 (40), 40.

(222) Pan, W.; Petersen, E.; Cai, N.; Ma, G.; Run Lee, J.; Feng, Z.; Liao, K.; Leong, K. Viscoelastic Properties of Human Mesenchymal Stem Cells. Proc. 2005 IEEE Eng. Med. Biol. 27th Annu. Conf. Shanghai, China, Sept. 1-4, 2005 2005, 5, 4854-4857.

(223) Fletcher, D. a.; Mullins, R. D. Cell Mechanics and the Cytoskeleton. Nature 2010, 463 (7280), 485-492. 
(224) Vichare, S.; Sen, S.; Inamdar, M. M. Cellular Mechanoadaptation to Substrate Mechanical Properties: Contributions of Substrate Stiffness and Thickness to Cell Stiffness Measurements Using AFM. Soft Matter 2014, 10 (8), 1174-1181.

(225) Radmacher, M.; Fritz, M.; Kacher, C. M. Measuring the Viscoelastic Properties of Human Platelets with the Atomic Force Microscope. Biophys. J. 1996, 70 (1), 556567.

(226) Dai, J.; Sheetz, M. P. Mechanical Properties of Neuronal Growth Cone Membranes Studied by Tether Formation with Laser Optical Tweezers. Biophys. J. 1995, 68 (3), 988-996.

(227) Dao, M.; Lim, C. T.; Suresh, S. Mechanics of the Human Red Blood Cell Deformed by Optical Tweezers. Journal of the Mechanics and Physics of Solids. November 2003, pp 2259-2280.

(228) Lim, C. T.; Zhou, E. H.; Quek, S. T. Mechanical Models for Living Cells - A Review. J. Biomech. 2006, 39 (2), 195-216.

(229) Song, F.; Ren, D. Stiffness of Cross-Linked Poly(dimethylsiloxane) Affects Bacterial Adhesion and Antibiotic Susceptibility of Attached Cells. Langmuir 2014, $30(34), 10354-10362$.

(230) Chuah, Y. J.; Kuddannaya, S.; Lee, M. H. A.; Zhang, Y.; Kang, Y. The Effects of Poly(dimethylsiloxane) Surface Silanization on the Mesenchymal Stem Cell Fate. Biomater. Sci. 2015, 3 (2), 383-390.

(231) Kuddannaya, S.; Chuah, Y. J. Y.; Hui, M.; Lee, A.; Menon, N. V; Kang, Y.; Zhang, Y. Surface Chemical Modification of Poly (Dimethylsiloxane) for the Enhanced 
Adhesion and Proliferation of Mesenchymal Stem Cells. ACS Appl. Mater. Interfaces 2013, 5 (19), 9777-9784.

(232) Lawton, R. A.; Price, C. R.; Runge, A. F.; Doherty, W. J.; Saavedra, S. S. Air Plasma Treatment of Submicron Thick PDMS Polymer Films: Effect of Oxidation Time and Storage Conditions. Colloids Surfaces A Physicochem. Eng. Asp. 2005, $253(1-3), 213-215$.

(233) Lee, S.; Vörös, J. An Aqueous-Based Surface Modification of Poly(dimethylsiloxane) with Poly(ethylene Glycol) to Prevent Biofouling. Langmuir 2005, 21 (25), 11957-11962.

(234) Wu, M.-H. Simple Poly(dimethylsiloxane) Surface Modification to Control Cell Adhesion. Surf. Interface Anal. 2009, 41 (1), 11-16.

(235) Nojehdehian, H.; Moztarzadeh, F.; Baharvand, H.; Mehrjerdi, N. Z.; Nazarian, H.; Tahriri, M. Effect of Poly-L-Lysine Coating on Retinoic Acid-Loaded PLGA Microspheres in the Differentiation of Carcinoma Stem Cells into Neural Cells. Int. J. Artif. Organs 2010, 33 (10), 721-730.

(236) Merkel, R.; Simson, R.; Simson, D. A.; Hohenadl, M.; Boulbitch, A.; Wallraff, E.; Sackmann, E. A Micromechanic Study of Cell Polarity and Plasma Membrane Cell Body Coupling in Dictyostelium. Biophys. J. 2000, 79 (2), 707-719.

(237) Daniel, M.; Gernald, S.; Winfield, S. Adhesion of Cells to Surfaces Coated with Polylysine. J. Cell Biol. 1975, 66, 198-200.

(238) Lu, H.; Guo, L.; Kawazoe, N.; Tateishi, T.; Chen, G. Effects of Poly(L-Lysine), Poly(acrylic Acid) and Poly(ethylene Glycol) on the Adhesion, Proliferation and 
Chondrogenic Differentiation of Human Mesenchymal Stem Cells. J. Biomater. Sci. Polym. Ed. 2009, 20 (5-6), 577-589.

(239) Somaiah, C.; Kumar, A.; Mawrie, D.; Sharma, A.; Patil, S. D.; Bhattacharyya, J.; Swaminathan, R.; Jaganathan, B. G. Collagen Promotes Higher Adhesion, Survival and Proliferation of Mesenchymal Stem Cells. PLoS One 2015, 10 (12), 1-15.

(240) Revel, J. P.; Hoch, P.; Ho, D. Adhesion of Culture Cells to Their Substratum. Exp. Cell Res. 1974, 84 (1-2), 207-218.

(241) Yu, H.; Tay, C. Y.; Leong, W. S.; Tan, S. C. W.; Liao, K.; Tan, L. P. Mechanical Behavior of Human Mesenchymal Stem Cells during Adipogenic and Osteogenic Differentiation. Biochem. Biophys. Res. Commun. 2010, 393 (1), 150-155.

(242) Shojaei-Baghini, E.; Zheng, Y.; Sun, Y. Automated Micropipette Aspiration of Single Cells. Ann. Biomed. Eng. 2013, 41 (6), 1208-1216.

(243) Theret, D. P.; Levesque, M. J.; Sato, M.; Nerem, R. M.; Wheeler, L. T. The Application of a Homogeneous Half-Space Model in the Analysis of Endothelial Cell Micropipette Measurements. J. Biomech. Eng. 1988, 110 (3), 190-199.

(244) Sato, M.; Theret, D. P.; Wheeler, L. T.; Ohshima, N.; Nerem, R. M. Application of the Micropipette Technique to the Measurement of Cultured Porcine Aortic Endothelial Cell Viscoelastic Properties. J. Biomech. Eng. 1990, 112 (3), 263-268.

(245) Sartori, S.; Chiono, V.; Tonda-Turo, C.; Mattu, C.; Gianluca, C. Biomimetic Polyurethanes in Nano and Regenerative Medicine. J. Mater. Chem. B 2014, 2 (32), 5128.

(246) Macrí-Pellizzeri, L.; Pelacho, B.; Sancho, A.; Iglesias-García, O.; Simón-Yarza, A. 
M.; Soriano-Navarro, M.; González-Granero, S.; García-Verdugo, J. M.; De-Juan-Pardo, E. M.; Prosper, F. Substrate Stiffness and Composition Specifically Direct Differentiation of Induced Pluripotent Stem Cells. Tissue Eng. Part A 2015, $21(9-10), 150318121612004$

(247) Tee, S.-Y.; Bausch, A. R. A.; Janmey, P. a. The Mechanical Cell. Curr. Biol. 2009, $19(17), 745-748$.

(248) Berdyyeva, T. K.; Woodworth, C. D.; Sokolov, I. Human Epithelial Cells Increase Their Rigidity with Ageing in Vitro : Direct Measurements. Phys. Med. Biol. 2005, $50(1), 81-92$.

(249) Maloney, J. M.; Nikova, D.; Lautenschläger, F.; Clarke, E.; Langer, R.; Guck, J.; Van Vliet, K. J. Mesenchymal Stem Cell Mechanics from the Attached to the Suspended State. Biophys. J. 2010, 99 (8), 2479-2487.

(250) Colley, H. E.; Mishra, G.; Scutt, A. M.; McArthur, S. L. Plasma Polymer Coatings to Support Mesenchymal Stem Cell Adhesion, Growth and Differentiation on Variable Stiffness Silicone Elastomers. Plasma Process. Polym. 2009, 6 (12), 831 839.

(251) Bongiorno, T.; Kazlow, J.; Mezencev, R.; Griffiths, S.; Olivares-Navarrete, R.; McDonald, J. F.; Schwartz, Z.; Boyan, B. D.; McDevitt, T. C.; Sulchek, T. Mechanical Stiffness as an Improved Single-Cell Indicator of Osteoblastic Human Mesenchymal Stem Cell Differentiation. J. Biomech. 2014, 47 (9), 2197-2204.

(252) Titushkin, I.; Cho, M. Modulation of Cellular Mechanics during Osteogenic Differentiation of Human Mesenchymal Stem Cells. Biophys. J. 2007, 93 (10), 
$3693-3702$.

(253) Yourek, G.; Hussain, M. A.; Mao, J. J. Cytoskeletal Changes of Mesenchymal Stem Cells during Differentiation. ASAIO J. 2007, 53 (2), 219-228.

(254) Yeung, T.; Georges, P. C.; Flanagan, L. a.; Marg, B.; Ortiz, M.; Funaki, M.; Zahir, N.; Ming, W.; Weaver, V.; Janmey, P. a. Effects of Substrate Stiffness on Cell Morphology, Cytoskeletal Structure, and Adhesion. Cell Motil. Cytoskeleton 2005, $60(1), 24-34$.

(255) Mao, A. S.; Shin, J. W.; Mooney, D. J. Effects of Substrate Stiffness and Cell-Cell Contact on Mesenchymal Stem Cell Differentiation. Biomaterials 2016, 98, 184191.

(256) Park, J. H.; Chung, B. G.; Lee, W. G.; Kim, J.; Brigham, M. D.; Shim, J.; Lee, S.; Hwang, C. M.; Durmus, N. G.; Demirci, U.; Khademhosseini, A. Microporous Cell-Laden Hydrogels for Engineered Tissue Constructs. Biotechnol. Bioeng. 2010, $106(1), 138-148$.

(257) Bosworth, L. A.; Rathbone, S. R.; Bradley, R. S.; Cartmell, S. H. Dynamic Loading of Electrospun Yarns Guides Mesenchymal Stem Cells towards a Tendon Lineage. J. Mech. Behav. Biomed. Mater. 2014, 39, 175-183.

(258) Woo, K. M.; Chen, V. J.; Ma, P. X. Nano-Fibrous Scaffolding Architecture Selectively Enhances Protein Adsorption Contributing to Cell Attachment. $J$. Biomed. Mater. Res. A 2003, 67 (2), 531-537.

(259) Dzenis, Y. Material Science. Spinning Continuous Fibers for Nanotechnology. Science 2004, 304 (5679), 1917-1919. 
(260) Yang, F.; Murugan, R.; Ramakrishna, S.; Wang, X.; Ma, Y. X.; Wang, S. Fabrication of Nano-Structured Porous PLLA Scaffold Intended for Nerve Tissue Engineering. Biomaterials 2004, 25 (10), 1891-1900.

(261) Murphy, W. L.; McDevitt, T. C.; Engler, A. J. Materials as Stem Cell Regulators. Nat. Mater. 2014, 13 (6), 547-557.

(262) Wade, R. J.; Bassin, E. J.; Gramlich, W. M.; Burdick, J. A. Nanofibrous Hydrogels with Spatially Patterned Biochemical Signals to Control Cell Behavior. Adv. Mater. 2015, $27(8), 1356-1362$.

(263) Kraehenbuehl, T. P.; Langer, R.; Ferreira, L. S. Three-Dimensional Biomaterials for the Study of Human Pluripotent Stem Cells. Nat. Methods 2011, 8 (9), 731-736.

(264) Xie, J.; Macewan, Ḱ. M. R.; Ray, Ḱ. W. Z.; Liu, W.; Siewe, D. Y.; Xia, Y. Radially Aligned, Electrospun Nanofibers and Tissue Regeneration Applications. ACS Nano 2010, 4 (9), 5027-5036.

(265) Dvir, T.; Timko, B. P.; Kohane, D. S.; Langer, R. Nanotechnological Strategies for Engineering Complex Tissues. Nat. Nanotechnol. 2011, 6 (1), 13-22.

(266) Krogman, K. C.; Lowery, J. L.; Zacharia, N. S.; Rutledge, G. C.; Hammond, P. T. Spraying Asymmetry into Functional Membranes Layer-by-Layer. Nat. Mater. 2009, $8(6), 512-518$.

(267) Jain, A.; Betancur, M.; Patel, G. D.; Valmikinathan, C. M.; Mukhatyar, V. J.; Vakharia, A.; Pai, S. B.; Brahma, B.; MacDonald, T. J.; Bellamkonda, R. V. Guiding Intracortical Brain Tumour Cells to an Extracortical Cytotoxic Hydrogel Using Aligned Polymeric Nanofibres. Nat. Mater. 2014, 13 (February), 308-316. 
(268) Onoe, H.; Okitsu, T.; Itou, A.; Kato-Negishi, M.; Gojo, R.; Kiriya, D.; Sato, K.; Miura, S.; Iwanaga, S.; Kuribayashi-Shigetomi, K.; Matsunaga, Y. T.; Shimoyama, Y.; Takeuchi, S. Metre-Long Cell-Laden Microfibres Exhibit Tissue Morphologies and Functions. Nat. Mater. 2013, 12 (6), 584-590.

(269) Lutolf, M. P.; Gilbert, P. M.; Blau, H. M. Designing Materials to Direct Stem-Cell Fate. Nature 2009, 462 (7272), 433-441.

(270) Lee, S.; Leach, M. K.; Redmond, S. A.; Chong, S. Y. C.; Mellon, S. H.; Tuck, S. J.; Feng, Z.-Q.; Corey, J. M.; Chan, J. R. A Culture System to Study Oligodendrocyte Myelination Processes Using Engineered Nanofibers. Nat. Methods 2012, 9 (9), 917-922.

(271) Kim, G.; Kim, W. Highly Porous 3D Nanofiber Scaffold Using an Electrospinning Technique. J. Biomed. Mater. Res. Part B Appl. Biomater. 2007, 81B (1), 104-110.

(272) Miller, J. S.; Stevens, K. R.; Yang, M. T.; Baker, B. M.; Nguyen, D.-H. T.; Cohen, D. M.; Toro, E.; Chen, A. a.; Galie, P. a.; Yu, X.; Chaturvedi, R.; Bhatia, S. N.; Chen, C. S. Rapid Casting of Patterned Vascular Networks for Perfusable Engineered Three-Dimensional Tissues. Nat. Mater. 2012, 11 (7), 768-774.

(273) Blakeney, B. A.; Tambralli, A.; Anderson, J. M.; Andukuri, A.; Lim, D. J.; Dean, D. R.; Jun, H. W. Cell Infiltration and Growth in a Low Density, Uncompressed Three-Dimensional Electrospun Nanofibrous Scaffold. Biomaterials 2011, 32 (6), $1583-1590$.

(274) Jin, L.; Wang, T.; Feng, Z.-Q.; Zhu, M.; Leach, M. K.; Naim, Y. I.; Jiang, Q. Fabrication and Characterization of a Novel Fluffy Polypyrrole Fibrous Scaffold 
Designed for 3D Cell Culture. J. Mater. Chem. 2012, 22 (35), 18321-18326.

(275) Si, Y.; Yu, J.; Tang, X.; Ge, J.; Ding, B. Ultralight Nanofibre-Assembled Cellular Aerogels with Superelasticity and Multifunctionality. Nat Commun 2014, 5, 5802.

(276) Bi, H.; Yin, Z.; Cao, X.; Xie, X.; Tan, C.; Huang, X.; Chen, B.; Chen, F.; Yang, Q.; Bu, X.; Lu, X.; Sun, L.; Zhang, H. Carbon Fiber Aerogel Made from Raw Cotton: A Novel, Efficient and Recyclable Sorbent for Oils and Organic Solvents. Adv. Mater. 2013, 25 (41), 5916-5921.

(277) Thorvaldsson, A.; Stenhamre, H.; Gatenholm, P.; Walkenstrom, P. Electrospinning of Highly Porous Scaffolds for Cartilage Regeneration. Biomacromolecules 2008, 9 (3), 1044-1049.

(278) Nakada Tetsuya;Iino, Yasuhiko;Katayama, Yasuo, H. Therapeutic Effects of the Long-Term Use of PAN Membrane Dialyzer in Hemodialysis Patients: Efficacy in Old Dialysis Patients with Mild PAD. J. Nippon Med. Sch. 2014, 81 (4), 221-235.

(279) Nataraj, S. K.; Yang, K. S.; Aminabhavi, T. M. Polyacrylonitrile-Based Nanofibers - A State-of-the-Art Review. Prog. Polym. Sci. 2012, 37 (3), 487-513.

(280) Pham, Q. P.; Sharma, U.; Mikos, A. G. Electrospinning of Polymeric Nanofibers for Tissue Engineering Applications: A Review. Tissue Eng. 2006, 12 (5), 11971211.

(281) Bhardwaj, N.; Kundu, S. C. Electrospinning: A Fascinating Fiber Fabrication Technique. Biotechnol. Adv. 2010, 28 (3), 325-347.

(282) Ishii, T.; Komizu, Y.; Shibuya, N.; Kusaka, K.; Matsushita, T. Effects of the Use of Polyacrylonitrile Nanofibers as 3D Scaffolds on the Growth and Function of 
Human Hepatoblasts Derived from Fetal Hepatocytes. Altern. to Anim. Test. Exp. 2015, $20(2), 66-72$.

(283) Truong, Y. B.; Glattauer, V.; Briggs, K. L.; Zappe, S.; Ramshaw, J. A. M. Collagen-Based Layer-by-Layer Coating on Electrospun Polymer Scaffolds. Biomaterials 2012, 33 (36), 9198-9204.

(284) Liu, S. P.; Lin, C. H.; Lin, S. J.; Fu, R. H.; Huang, Y. C.; Chen, S. Y.; Lin, S. Z.; Hsu, C. Y.; Shyu, W. C. Electrospun Polyacrylonitrile-Based Nanofibers Maintain Embryonic Stem Cell Stemness via TGF-Beta Signaling. J. Biomed. Nanotechnol. 2016, 12 (4), 732-742.

(285) Han, D.; Boyce, S. T.; Steckl, A. J. Versatile Core-Sheath Biofibers Using Coaxial Electrospinning. MRS Proc. 2011, 1094, 1094-NaN-2.

(286) George, P. M.; Lyckman, A. W.; Lavan, D. a.; Hegde, A.; Leung, Y.; Avasare, R.; Testa, C.; Alexander, P. M.; Langer, R.; Sur, M. Fabrication and Biocompatibility of Polypyrrole Implants Suitable for Neural Prosthetics. Biomaterials 2005, 26 (17), $3511-3519$.

(287) Wang, X.; Gu, X.; Yuan, C.; Chen, S.; Zhang, P.; Zhang, T.; Yao, J.; Chen, F.; Chen, G. Evaluation of Biocompatibility of Polypyrrole in Vitro and in Vivo. J. Biomed. Mater. Res. A 2004, 68 (3), 411-422.

(288) Xie, J.; MacEwan, M. R.; Willerth, S. M.; Li, X.; Moran, D. W.; Sakiyama-Elbert, S. E.; Xia, Y. Conductive Core-Sheath Nanofibers and Their Potential Application in Neural Tissue Engineering. Adv. Funct. Mater. 2009, 19 (14), 2312-2318.

(289) Jin, L.; Xu, Q.; Wu, S.; Kuddannaya, S.; Li, C.; Huang, J.; Zhang, Y.; Wang, Z. 
Synergistic Effects of Conductive Three-Dimensional Nanofibrous Microenvironments and Electrical Stimulation on the Viability and Proliferation of Mesenchymal Stem Cells. ACS Biomater. Sci. Eng. 2016, 2 (11), 2042-2049.

(290) MacQueen, L.; Sun, Y.; Simmons, C. a. Mesenchymal Stem Cell Mechanobiology and Emerging Experimental Platforms. J. R. Soc. Interface 2013, 10 (84), 20130179.

(291) Panadero, J. A.; Sencadas, V.; Silva, S. C. M.; Ribeiro, C.; Correia, V.; Gama, F. M.; Gomez Ribelles, J. L.; Lanceros-Mendez, S. Mechanical Fatigue Performance of PCL-Chondroprogenitor Constructs after Cell Culture under Bioreactor Mechanical Stimulus. J. Biomed. Mater. Res. - Part B Appl. Biomater. 2016, 104 (2), 330-338.

(292) Baker, B. M.; Shah, R. P.; Huang, A. H.; Mauck, R. L. Dynamic Tensile Loading Improves the Functional Properties of Mesenchymal Stem Cell-Laden Nanofiber-Based Fibrocartilage. Tissue Eng. Part A 2011, 17 (9-10), 1445-1455.

(293) Faten, A. M.; Abo-Aziza; Zaki, A. A. Confluence-Associated Proliferation and Osteogenic Differentiation of Bone Marrow Mesenchymal Stem Cell (BMMSCs). Int. Res. J. Biol. Sci. 2016, 5 (5), 44-56.

(294) Both, S. K.; van der Muijsenberg, A. J. C.; van Blitterswijk, C. A.; de Boer, J.; de Bruijn, J. D. A Rapid and Efficient Method for Expansion of Human Mesenchymal Stem Cells. Tissue Eng. 2007, 13 (1), 3-9.

(295) Bonani, W.; Maniglio, D.; Motta, A.; Tan, W.; Migliaresi, C. Biohybrid Nanofiber Constructs with Anisotropic Biomechanical Properties. J. Biomed. Mater. Res. - 
Part B Appl. Biomater. 2011, 96 B (2), 276-286.

(296)

Zhang, C. SYNTHESIS AND CHARACTERIZATION OF BIODEGRADABLE POLYURETHANES FOR BIOMEDICAL APPLICATION, Clemson University, 2008.

(297) Vincent, J. Basic Elasticity and Viscoelasticity. In Structural biomaterials; Princeton University Press, 2012; pp 1-28.

(298) Kearney, E. M.; Farrell, E.; Prendergast, P. J.; Campbell, V. A. Tensile Strain as a Regulator of Mesenchymal Stem Cell Osteogenesis. Ann. Biomed. Eng. 2010, 38 (5), 1767-1779.

(299) HUANG, C.; HAGAR, K. L.; FROST, L. E.; SUN, Y.; CHEUNG, H. S. Effects of Cyclic Compressive Loading on Chondrogenesis of Rabbit Bone-Marrow Derived Mesenchymal Stem Cells. Stem Cells 2003, No. 22, 313-323.

(300) Huang, C.-H.; Chen, M.-H.; Young, T.-H.; Jeng, J.-H.; Chen, Y.-J. Interactive Effects of Mechanical Stretching and Extracellular Matrix Proteins on Initiating Osteogenic Differentiation of Human Mesenchymal Stem Cells. J. Cell. Biochem. 2009, 108 (August), 1263-1273.

(301) Visser, J.; Melchels, F. P. W.; Jeon, J. E.; van Bussel, E. M.; Kimpton, L. S.; Byrne, H. M.; Dhert, W. J. a; Dalton, P. D.; Hutmacher, D. W.; Malda, J. Reinforcement of Hydrogels Using Three-Dimensionally Printed Microfibres. Nat. Commun. 2015, 6, 6933.

(302) Shi, Y.; Li, H.; Zhang, X.; Fu, Y.; Huang, Y.; Lui, P. P. Y.; Tang, T.; Dai, K. Continuous Cyclic Mechanical Tension Inhibited Runx2 Expression in 
Mesenchymal Stem Cells through RhoA-ERK1/2 Pathway. J. Cell. Physiol. 2011, $226(8), 2159-2169$.

(303) Weightman, A.; Jenkins, S.; Pickard, M.; Chari, D.; Yang, Y. Alignment of Multiple Glial Cell Populations in 3D Nanofiber Scaffolds: Toward the Development of Multicellular Implantable Scaffolds for Repair of Neural Injury. Nanomedicine Nanotechnology, Biol. Med. 2014, 10 (2), 291-295.

(304) Pelto, J.; Björninen, M.; Pälli, A.; Talvitie, E.; Hyttinen, J.; Mannerström, B.; Suuronen Seppanen, R.; Kellomäki, M.; Miettinen, S.; Haimi, S. Novel Polypyrrole-Coated Polylactide Scaffolds Enhance Adipose Stem Cell Proliferation and Early Osteogenic Differentiation. Tissue Eng. Part A 2013, 19 (7-8), 882-892.

(305) Bandeira, F.; Lent, R.; Herculano-Houzel, S. Changing Numbers of Neuronal and Non-Neuronal Cells Underlie Postnatal Brain Growth in the Rat. Proc. Natl. Acad. Sci. U. S. A. 2009, 106 (33), 14108-14113.

(306) Nisbet, D. R.; Forsythe, J. S.; Shen, W.; Finkelstein, D. I.; Horne, M. K. Review Paper: A Review of the Cellular Response on Electrospun Nanofibers for Tissue Engineering. J. Biomater. Appl. 2009, 24 (1), 7-29.

(307) Subramanian, A.; Krishnan, U.; Sethuraman, S. Development of Biomaterial Scaffold for Nerve Tissue Engineering: Biomaterial Mediated Neural Regeneration. J. Biomed. Sci. 2009, $16(1), 108$.

(308) Kumbar, S. G.; James, R.; Nukavarapu, S. P.; Laurencin, C. T. Electrospun Nanofiber Scaffolds: Engineering Soft Tissues. Biomed. Mater. 2008, 3 (3), 34002.

(309) Xie, J.; MacEwan, M. R.; Schwartz, A. G.; Xia, Y. Electrospun Nanofibers for 
Neural Tissue Engineering. Nanoscale 2010, 2 (1), 35-44.

(310) Bakhru, S.; Nain, A. S.; Highley, C.; Wang, J.; Campbell, P.; Amon, C.; Zappe, S. Direct and Cell Signaling-Based, Geometry-Induced Neuronal Differentiation of Neural Stem Cells. Integr. Biol. 2011, 3 (12), 1207-1214.

(311) Mammadov, B.; Sever, M.; Guler, M. O.; Tekinay, A. B. Neural Differentiation on Synthetic Scaffold Materials. Biomater. Sci. 2013, 1 (11), 1119-1137.

(312) Kabiri, M.; Soleimani, M.; Shabani, I.; Futrega, K.; Ghaemi, N.; Ahvaz, H. H.; Elahi, E.; Doran, M. R. Neural Differentiation of Mouse Embryonic Stem Cells on Conductive Nanofiber Scaffolds. Biotechnol. Lett. 2012, 34 (7), 1357-1365.

(313) McMurtrey, R. J. Patterned and Functionalized Nanofiber Scaffolds in Three-Dimensional Hydrogel Constructs Enhance Neurite Outgrowth and Directional Control. J. Neural Eng. 2014, 11 (6), 66009.

(314) Çapkın, M.; Çakmak, S.; Kurt, F. Ö.; Gümüşderelioğlu, M.; Şen, B. H.; Türk, B. T.; Deliloğlu-Gürhan, S. İ. Random/aligned Electrospun PCL/PCL-Collagen Nanofibrous Membranes: Comparison of Neural Differentiation of Rat AdMSCs and BMSCs. Biomed. Mater. 2012, 7 (4), 45013.

(315) Xie, J.; MacEwan, M. R.; Li, X.; Sakiyama-Elbert, S. E.; Xia, Y. Neurite Outgrowth on Nanofiber Scaffolds with Different Orders, Structures, and Surface Properties. ACS Nano 2009, 3 (5), 1151-1159.

(316) Wang, H. B.; Mullins, M. E.; Cregg, J. M.; Hurtado, A.; Oudega, M.; Trombley, M. T.; Gilbert, R. J. Creation of Highly Aligned Electrospun Poly-L-Lactic Acid Fibers for Nerve Regeneration Applications. J. Neural Eng. 2009, 6 (1), 16001. 
(317) Xie, J.; Willerth, S. M.; Li, X.; Macewan, M. R.; Rader, A.; Sakiyama-Elbert, S. E.; Xia, Y. The Differentiation of Embryonic Stem Cells Seeded on Electrospun Nanofibers into Neural Lineages. Biomaterials 2009, 30 (3), 354-362.

(318) Tian, B.; Liu, J.; Dvir, T.; Jin, L.; Tsui, J. H.; Qing, Q.; Suo, Z.; Langer, R.; Kohane, D. S.; Lieber, C. M. Macroporous Nanowire Nanoelectronic Scaffolds for Synthetic Tissues. Nat. Mater. 2012, 11 (11), 986-994.

(319) Dvir, T.; Timko, B. P.; Brigham, M. D.; Naik, S. R.; Karajanagi, S. S.; Levy, O.; Jin, H.; Parker, K. K.; Langer, R.; Kohane, D. S. Nanowired Three-Dimensional Cardiac Patches. Nat. Nanotechnol. 2011, 6 (11), 720-725.

(320) Liu, Y.; Liu, X.; Chen, J.; Gilmore, K. J.; Wallace, G. G. 3D Bio-Nanofibrous PPy/SIBS Mats as Platforms for Cell Culturing. Chem. Commun. 2008, No. 32, 3729.

(321) Banker, G. A.; Cowan, W. M. Rat Hippocampal Neurons in Dispersed Cell Culture. Brain Res. 1977, 126 (3), 342-397.

(322) Brewer, G. J. Isolation and Culture of Adult Rat Hippocampal Neurons. J. Neurosci. Methods 1997, 71 (2), 143-155.

(323) Fan, Y.; Xu, F.; Huang, G.; Lu, T. J.; Xing, W. Single Neuron Capture and Axonal Development in Three-Dimensional Microscale Hydrogels. Lab Chip 2012, 12 (22), $4724-4731$.

(324) Kuddannaya, S.; Bao, J.; Zhang, Y. Enhanced in Vitro Biocompatibility of Chemically Modified Poly(dimethylsiloxane) Surfaces for Stable Adhesion and Long-Term Investigation of Brain Cerebral Cortex Cells. ACS Appl. Mater. 
Interfaces 2015, 7 (45), 25529-25538.

(325) Roessmann, U.; Velasco, M. E.; Sindely, S. D.; Gambetti, P. Glial Fibrillary Acidic Protein (GFAP) in Ependymal Cells during Development. An Immunocytochemical Study. Brain Res. 1980, 200 (1), 13-21.

(326) Hol, E. M.; Pekny, M. Glial Fibrillary Acidic Protein (GFAP) and the Astrocyte Intermediate Filament System in Diseases of the Central Nervous System. Curr. Opin. Cell Biol. 2015, 32, 121-130.

(327) Brandt, R. The Tau Proteins in Neuronal Growth and Development. Front Biosci 1996, 1 (August), d118--d130.

(328) Sahoo, S.; Dhibar, S.; Das, C. K. Facile Synthesis of Polypyrrole Nanofiber and Its Enhanced Electrochemical Performances in Different Electrolytes. Express Polym. Lett. 2012, 6 (12), 965-974.

(329) Castano, H.; O’Rear, E. A.; McFetridge, P. S.; Sikavitsas, V. I. Polypyrrole Thin Films Formed by Admicellar Polymerization Support the Osteogenic Differentiation of Mesenchymal Stem Cells. Macromol. Biosci. 2004, 4 (8), 785794.

(330) Noctor, S. C.; Flint, A. C.; Weissman, T. A.; Dammerman, R. S.; Kriegstein, A. R. Neurons Derived from Radial Glial Cells Establish Radial Units in Neocortex. Nature 2001, 409 (6821), 714-720.

(331) Price, J.; Thurlow, L. Cell Lineage in the Rat Cerebral Cortex: A Study Using Retroviral-Mediated Gene Transfer. Development 1988, 104 (3), 473-482.

(332) Araque, A.; Navarrete, M. Glial Cells in Neuronal Network Function. Philos. Trans. 
R. Soc. B 2010, 365, 2375-2381.

(333) Odawara, A.; Gotoh, M.; Suzuki, I. A Three-Dimensional Neuronal Culture Technique That Controls the Direction of Neurite Elongation and the Position of Soma to Mimic the Layered Structure of the Brain. RSC Adv. 2013, 3 (45), 2362023630.

(334) Longair, M. H.; Baker, D. A.; Armstrong, J. D. Simple Neurite Tracer: Open Source Software for Reconstruction, Visualization and Analysis of Neuronal Processes. Bioinformatics 2011, 27 (17), 2453-2454.

(335) Barres, B. A. The Mystery and Magic of Glia: A Perspective on Their Roles in Health and Disease. Neuron 2008, 60 (3), 430-440.

(336) Gordon, J.; Amini, S.; White, M. K. General Overview of Neuronal Cell Culture. In Methods in Molecular Biology; 2013; Vol. 1078, pp 1-8.

(337) Hernández-Ferrer, J.; Pérez-Bruzón, R. N.; Azanza, M. J.; González, M.; Del Moral, R.; Ansón-Casaos, A.; de la Fuente, J. M.; Marijuan, P. C.; Martínez, M. T. Study of Neuron Survival on Polypyrrole-Embedded Single-Walled Carbon Nanotube Substrates for Long-Term Growth Conditions. J. Biomed. Mater. Res. Part A 2014, $102(12), 4443-4454$.

(338) Jin, L.; Yue, D.; Xu, Z.-W.; Liang, G.; Zhang, Y.; Zhang, J.-F.; Zhang, X.; Wang, Z. Fabrication, Mechanical Properties, and Biocompatibility of Reduced Graphene Oxide-Reinforced Nanofiber Mats. RSC Adv. 2014, 4 (66), 35035-35041.

(339) Ravi, M.; Paramesh, V.; Kaviya, S. R.; Anuradha, E.; Paul Solomon, F. D. 3D Cell Culture Systems: Advantages and Applications. J. Cell. Physiol. 2015, 230 (1), 16- 
26.

(340) Liu, J.; Appaix, F.; Bibari, O.; Marchand, G.; Benabid, A.-L.; Sauter-Starace, F.; Waard, M. De. Control of Neuronal Network Organization by Chemical Surface Functionalization of Multi-Walled Carbon Nanotube Arrays. Nanotechnology 2011, $22(19), 195101$.

(341) Nicolau, D. V.; Taguchi, T.; Taniguchi, H.; Tanigawa, H.; Yoshikawa, S. Patterning Neuronal and Glia Cells on Light-Assisted Functionalised Photoresists. Biosens. Bioelectron. 1999, 14 (3), 317-325.

(342) Kølvraa, M.; Müller, F. C.; Jahnsen, H.; Rekling, J. C. Mechanisms Contributing to Cluster Formation in the Inferior Olivary Nucleus in Brainstem Slices from Postnatal Mice. J. Physiol. 2014, 592, 33-47.

(343) Stewart, E.; Kobayashi, N. R.; Higgins, M. J.; Quigley, A. F.; Jamali, S.; Moulton, S. E.; Kapsa, R. M. I.; Wallace, G. G.; Crook, J. M. Electrical Stimulation Using Conductive Polymer Polypyrrole Promotes Differentiation of Human Neural Stem Cells: A Biocompatible Platform for Translational Neural Tissue Engineering. Tissue Eng. Part C. Methods 2015, 21 (4), 385-393.

(344) Dhar, D. EXPLORING ELECTRIC FIELD-INDUCED CHANGES IN ASTROCYTE BEHAVIOR, Virginia Commonwealth University, 2013.

(345) Kotwal, A.; Schmidt, C. E. Electrical Stimulation Alters Protein Adsorption and Nerve Cell Interactions with Electrically Conducting Biomaterials. Biomaterials 2001, $22(10), 1055-1064$.

(346) Eroglu, C. The Role of Astrocyte-Secreted Matricellular Proteins in Central 
Nervous System Development and Function. J. Cell Commun. Signal. 2009, 3 (34), 167-176.

(347) Vats, K.; Benoit, D. S. W. Dynamic Manipulation of Hydrogels to Control Cell Behavior: A Review. Tissue Eng. Part B. Rev. 2013, 19 (6), 455-469.

(348) McCaig, C. D.; Rajnicek, A. M.; Song, B.; Zhao, M. Controlling Cell Behavior Electrically: Current Views and Future Potential. Physiol. Rev. 2005, 85 (3), $943-$ 978.

(349) McCullen, S. D.; McQuilling, J. P.; Grossfeld, R. M.; Lubischer, J. L.; Clarke, L. I.; Loboa, E. G. Application of Low-Frequency Alternating Current Electric Fields via Interdigitated Electrodes: Effects on Cellular Viability, Cytoplasmic Calcium, and Osteogenic Differentiation of Human Adipose-Derived Stem Cells. Tissue Eng. Part C. Methods 2010, 16 (6), 1377-1386.

(350) Yow, S.-Z.; Lim, T. H.; Yim, E. K. F.; Lim, C. T.; Leong, K. W. A 3D Electroactive Polypyrrole-Collagen Fibrous Scaffold for Tissue Engineering. Polymers (Basel). 2011, $3(1), 527-544$.

(351) Azevedo, F. A. C.; Carvalho, L. R. B.; Grinberg, L. T.; Farfel, J. M.; Ferretti, R. E. L.; Leite, R. E. P.; Filho, W. J.; Lent, R.; Herculano-Houzel, S. Equal Numbers of Neuronal and Nonneuronal Cells Make the Human Brain an Isometrically Scaled-up Primate Brain. J. Comp. Neurol. 2009, 513 (5), 532-541.

(352) Kwok, C. B.; Ho, F. C.; Li, C. W.; Ngan, a. H. W.; Chan, D.; Chan, B. P. Compression-Induced Alignment and Elongation of Human Mesenchymal Stem Cell (hMSC) in 3D Collagen Constructs Is Collagen Concentration Dependent. $J$. 
Biomed. Mater. Res. - Part A 2013, 101 A (6), 1716-1725.

(353) Pelaez, D.; Huang, C.-Y. C.; Cheung, H. S. Cyclic Compression Maintains Viability and Induces Chondrogenesis of Human Mesenchymal Stem Cells in Fibrin Gel Scaffolds. Stem Cells Dev. 2009, 18 (1), 93-102. 